\title{
Neuronal Circuit Dissection in the Drosophila Brain: the Role of Serotonin-Releasing Neurons in Arousal
}

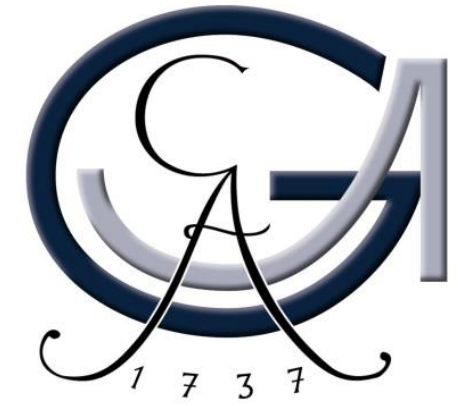

Dissertation

for the award of the degree

"Doctor rerum naturalium"

of the Georg-August-Universität Göttingen

within the doctoral program Sensory and Motor Neuroscience

of the Göttingen Graduate School for Neurosciences, Biophysics, and Molecular

Biosciences (GGNB)

of the Georg-August University School of Science (Gauss)

submitted by

Atefeh Pooryasin

from

Shiraz, Iran

Göttingen, 2014 


\section{PhD Committee Member:}

Prof. Dr. André Fiala (Supervisor, Reviewer)

Dept. of Molecular Neurobiology of Behavior

Johann-Friedrich-Blumenbach-Institute for Zoology and Anthropology,

Georg-August-University Göttingen

PhD Committee Member:

Prof. Dr. Martin Göpfert (Reviewer)

Dept. of Cellular Neurobiology

Georg-August-University Göttingen

PhD Committee Member:

Prof. Dr. Julia Fischer

Dept. of Cognitive Ethology

German Primate Center

Georg-August-University Göttingen

Thesis submission: April $30^{\text {th }}, 2014$ 


\section{Declaration of academic honesty:}

I hereby declare that the PhD thesis entitled "Neuronal Circuit Dissection in the Drosophila Brain: the Role of Serotonin-Releasing Neurons in Arousal" has been written independently and with no other sources and aids other than quoted within texts, references and acknowledgments.

Atefeh Pooryasin

Göttingen, April 30 ${ }^{\text {th }}, 2014$ 


\section{Table of contents}

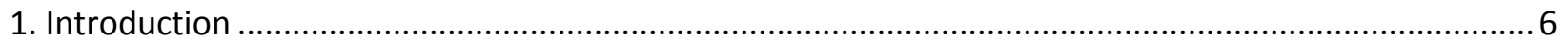

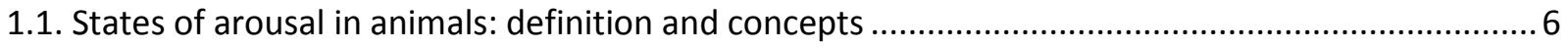

1.2. The neuromodulatory effect of serotonin on arousal states in vertebrates and invertebrates ....... 8

1.3. Drosophila melanogaster as a model organism .......................................................................... 9

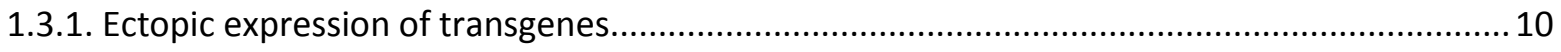

1.3.2. FLP-FRT -mediated site-directed recombination .............................................................. 14

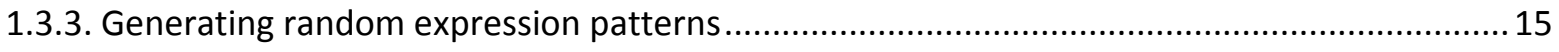

1.3.4. Intersectional strategies to restrict gene expression patterns ............................................ 16

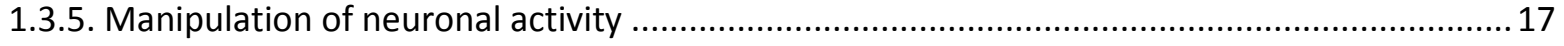

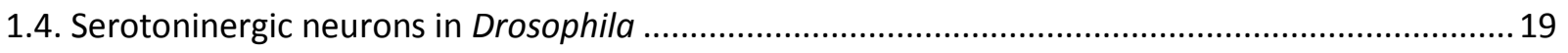

1.4.1. Serotonin biosynthesis, transportation, metabolism and receptors ..................................... 19

1.4.2. The distribution of serotonin-releasing neurons in the Drosophila brain ............................. 22

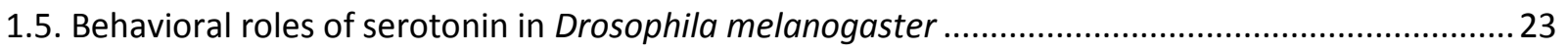

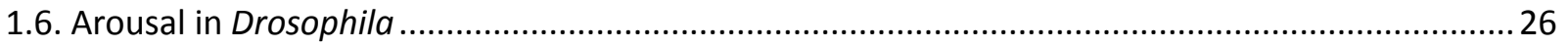

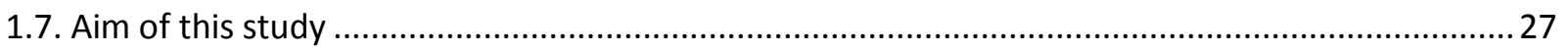

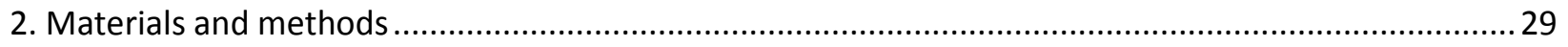

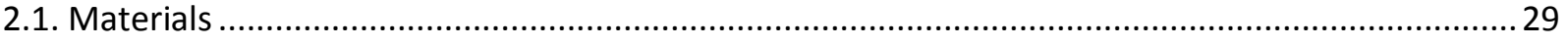

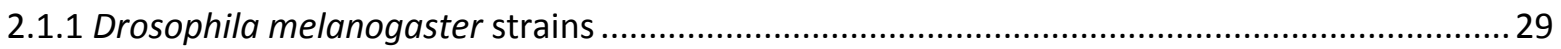

2.1.2. Gal4 lines from Janelia stock collection ............................................................................ 31

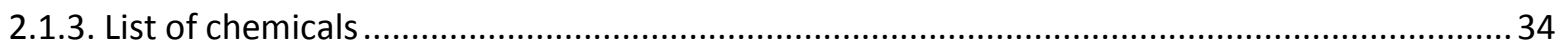

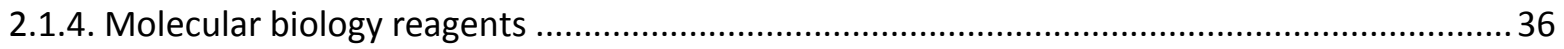

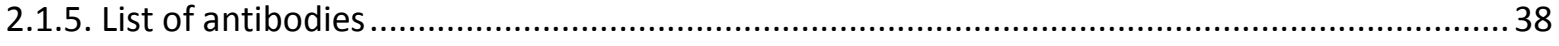

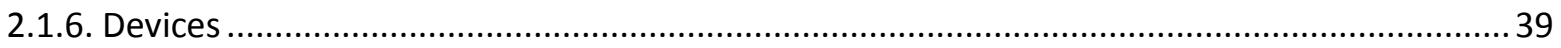

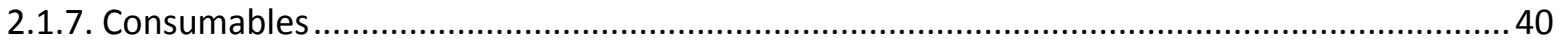

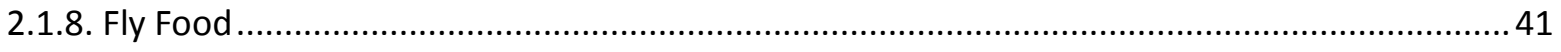

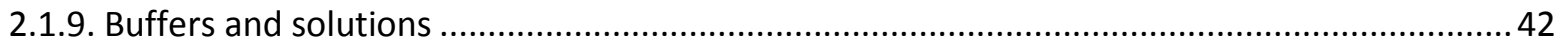


2.2.1 Fly maintenance

2.2.2. Generating transgenic flies

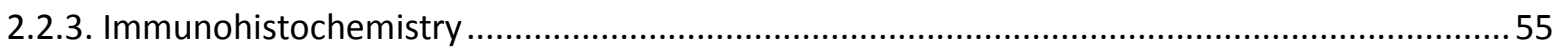

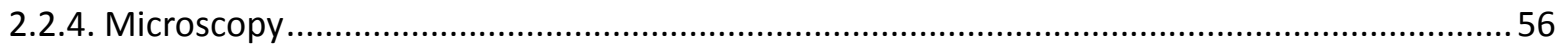

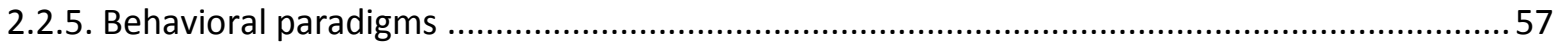

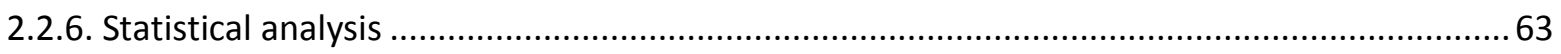

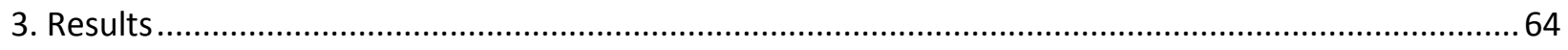

3.1. The distribution of serotoninergic cells in the Drosophila brain .................................................64

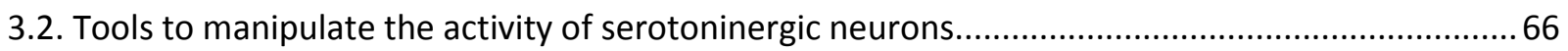

3.2.1. Generating a tool for thermogenetic neuronal activation .................................................. 66

3.2.2. Anatomical comparison of the expression pattern induced by three tryptophan hydroxylase

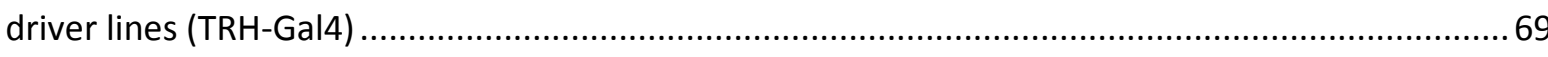

3.3. Effect of thermogenetically activating serotoninergic neurons on the arousal state in Drosophila

3.3.1. Thermogenetic activation of serotoninergic neurons leads to a significant reduction in the walking velocity.

3.3.2. Activating serotoninergic neurons in the brain is sufficient for the reduction in the walking velocity .73

3.3.3. The effect of thermogenetic activation of serotoninergic neurons on the animals' responsiveness to external mechanical stimuli ............................................................................... 75

3.3.4. Thermogenetic activation of serotoninergic neurons prevents starved flies from eating ........ 82

3.4. Thermogenetic blocking transmission from serotoninergic neurons increases walking velocity ... 83

3.5. Regulatory effect of serotoninergic neurons on the endogenous arousal state............................ 84

3.6. Is it the neurotransmitter serotonin that is involved in the regulation of arousal state? ...............87

3.7. Identification of serotoninergic neurons underlying the modulation of arousal state in Drosophila

3.7.1. Activation of restricted number of serotoninergic cells using a stochastic approach..............90

3.7.2. An intersectional approach for expressing mCherry-dTRPA1 in different subsets of serotoninergic neurons. 101 


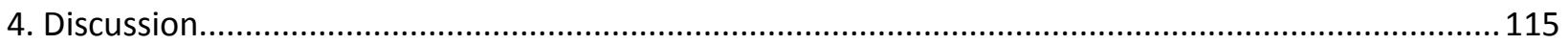

4.1. A thermogenetic approach to dissect serotonin-dependent arousal in Drosophila ....................116

4.2. Serotoninergic neurons: a modulator of the general arousal level in Drosophila ........................117

4.3. Conclusions and limitations using pharmacological approaches ............................................... 119

4.4. Sufficiency and necessity of serotonergic neurons to modulate the arousal level ......................120

4.5. Serotoninergic neurons: modulator of sleep/activity states .................................................. 121

4.6. Dissecting the neuronal circuits underlying modulation of arousal ......................................... 122

4.7. Identification of specific serotoninergic neurons underlying the modulation of arousal state in

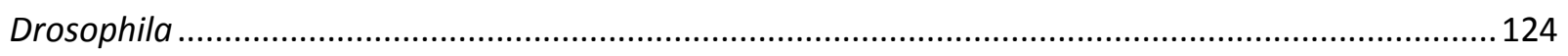

4.8. Interaction of serotonin and other aminergic systems in the regulation of arousal.................... 125

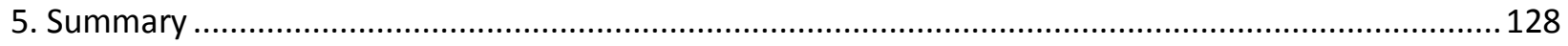

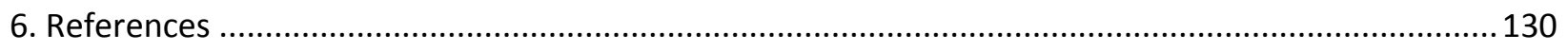

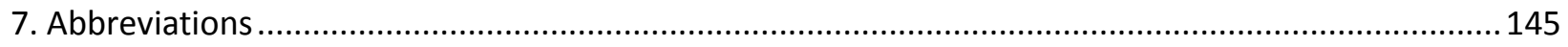

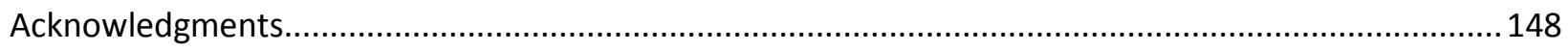




\section{Introduction}

\subsection{States of arousal in animals: definition and concepts}

Animals as well as humans show states of high overall activity and states of rest. This is most obvious in circadian sleep-wake cycles. However, intrinsic motivational factors, e.g., hunger or thirst, influence the activity state of the animal as well. External stimuli, e.g., hazardous smells or painful stimuli can also cause a drastic increase in activity and in responsiveness, which is referred to as sensitization. These phenomena have led to the concept of "arousal", a central state of the animal that underlies its self-generated, internal activity and/or responsiveness to environmental stimuli. Here, arousal is referred to as such a physiological state of the animal in which sleep and wakefulness represent the extreme ends (Coull, 1998). Further, the arousal level can change within a wakeful period, reflected by the differential responsiveness of an animal towards external stimuli (Van Swinderen and Andretic, 2011). A hungry animal, for example, will not only be more active in order to enhance the probability to find food, but will also be more sensitive to sensory stimuli that signal food sources. Arousal is, therefore, regarded to be an internal factor that influences the initiation and execution of different behaviors. As a relatively general concept, an optimal arousal level is a prerequisite for the motivation and readiness for energizing a certain performance (Hebb, 1955). Despite much effort to unravel the neurophysiological mechanisms underlying arousal, it still remains unanswered whether the arousal system is based on a single and one-dimensional physiological factor controlling and potentiating all kinds of behavior (Hebb, 1955), or whether multiple and task-specific arousal systems regulate distinct behaviors (Robbins, 1997; reviewed by Berlucchi, 1997; reviewed by Jing et al., 2009). As one example, according to the Moruzzi theory (1958), the ascending reticular system in the brainstem of mammals was considered to represent an arousal system controlling multiple behavioral tasks (reviewed by Berlucchi, 1997). However, on the basis of the diverse anatomical and physiological properties of the brainstem, a theory of multiple and specific arousal system was proposed (Robbins, 1997). Both concepts, single and multiple arousal systems, are schematically illustrated in figure $1.1(A, B)$. 
A

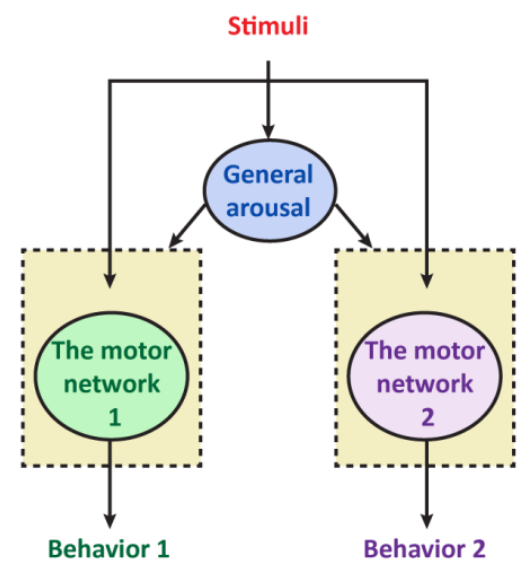

B

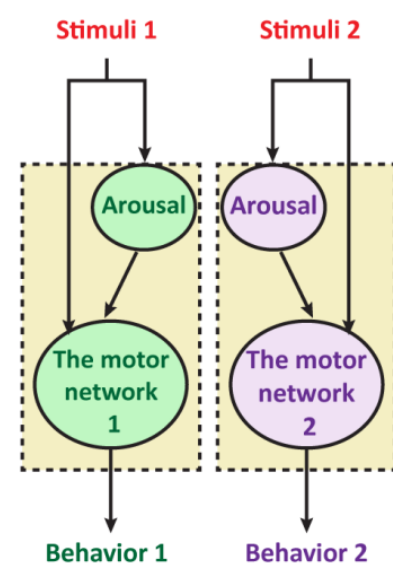

C

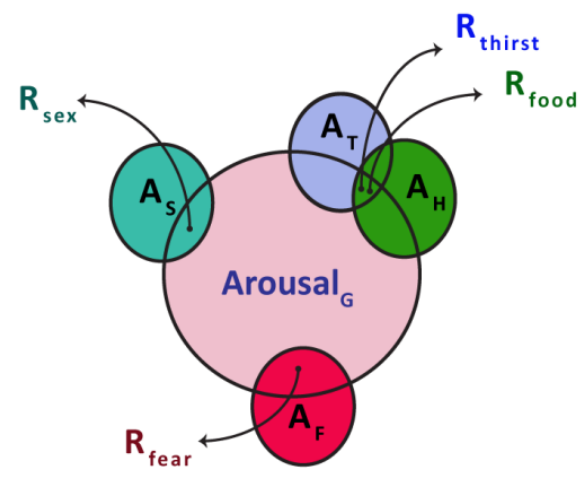

Figure 1.1. Schematic illustration of different arousal theories. (A) Single arousal system which modulates various kinds of behavior. (B) Multiple arousal systems which are task-specific. (C) Arousal system consisting of two components. General arousal (Arousal $\mathrm{G}_{G}$ ) and task-specific arousal systems like hunger-induced arousal $\left(A_{H}\right)$, thirstinduced arousal $\left(A_{t}\right)$, sex-induced arousal $\left(A_{s}\right)$ and fear-induced arousal $\left(A_{F}\right)$, which motivate related responses $\left(R_{\text {food }}, R_{\text {thirst }}, R_{\text {sex }}\right.$ and $\left.R_{\text {fear }}\right)$. Modified from Jing et al., 2009 and Pfaff et al., 2008.

Pfaff et al. (2008) merged the two models and proposed that an overall arousal system must consist of both general and specific (or localized) compartments. In this model a general arousal system exerts its influence on multiple classes of behavior, but specific arousal systems each initiate one specific type or class of behavior (reviewed by Jing et al., 2009; Figure 1.1. C).

Despite the differences and conceptual elusiveness of these hypotheses, it is clear that states of arousal are in many cases regulated by the action of modulatory transmitters, both in vertebrates and invertebrates. The modulatory effect of different biogenic amines, like dopamine, serotonin or norepinephrine, on arousal has been shown in a large amount of studies across many species (e.g., Rosen et al., 1989; Hildebrandt et al., 2010; Robbins et al., 1998; Andretic et al., 2005; Devitze et al., 2006). 


\subsection{The neuromodulatory effect of serotonin on arousal states in vertebrates and invertebrates}

The effect of serotoninergic neurons on regulating arousal states has been shown in a large variety of different species across the animal kingdom. In molluscs, serotoninergic neurons are broadly distributed across the ganglia of the nervous system and are involved in the regulation of different motor networks initiating behaviors like feeding, escape swim or turning behavior (Gillette, 2006). A model of arousal mechanisms in gastropods has been proposed by Jing et al. (2009). Here, serotoninergic interneurons are thought to act as general arousal components that interact with specific (localized) arousal elements for modulating different behaviors. Further, in the mollusc Aplysia it has been shown that serotoninergic metacerebral cells (MCCs) in the cerebral ganglion regulate food-induced arousal. This kind of arousal in Aplysia is naturally evoked by food stimuli and is behaviorally characterized by an increase in biting magnitude and rate. Although the MCCs innervate the muscles, firing of them at physiological rates does not induce any muscle contraction. Destroying the MCCs affects the rate and magnitude of consummatory biting responses but does not cause any deficit in the non-feeding behavior and also not in appetitive, goal-directed feeding responses (Rosen et al., 1989).

The neuromodulatory effect of serotonin on different feeding states in another invertebrate species, C. elegans, was shown by Flavell et al. (2013). Depending on the amount of the food in the arena and on the arousal state of the animal, $C$. elegans switches between two different foraging states called roaming and dwelling. Flavell et al. (2013) found that serotonin has a modulatory effect on this arousal-dependent exploration behavior in C. elegans: Activation of serotoninergic cells promotes the dwelling states through the MOD-1 serotonin-gated chloride channel (Flavell et al., 2013).

In mammals, serotonin-producing neurons are concentrated in the dorsal raphe nuclei (DR) in the midbrain, and their fibers project to the cerebral cortex, the hippocampus, the limbic system, and the hypothalamus. Here, serotonin has been shown to modulate fear and anxietyrelated behaviors (Lowry et al., 2005; Lowry et al., 2008; Hale et al., 2012; Paul et al., 2011), food intake (Halford and Blundell; 1996), aggression (review by Haller, 2013) and learning and memory (Gasbarri and Pompili, 2014). In addition, serotonin promotes wakefulness and inhibits rapid eye movement (REM) sleep (Jacobs and Fornal, 1999; Saper et al., 2010; Monti, 2011). 
Serotonin controls different behaviors through different receptors located in both, pre- or postsynapses of neurons, and can be both, inhibitory or excitatory (review by Fox and Lowry, 2013).

Finally, another aspect of arousal should be mentioned: A hungry animal should stop to actively search once it has found the food and its motivation to eat should decrease with increasing satiation. For an appropriate switch of behavior, the mechanism that decreases the arousal level is biologically as important as the mechanism that increases it. In mammals it has been shown that serotonin exerts a modulatory effect on the termination of eating through the 5HT1A receptor. Dysregulation of this receptor in the hippocampus causes a difficulty to stop eating (Hildebrandt et al., 2010). Overall, there is a plethora of evidence that the biogenic amine serotonin as a modulatory transmitter is involved in the modulation of more or less specific behavioral actions and responses across many animal species. In some "simple" animal models, e.g., Aplysia or C. elegans, the function of distinct, individual serotoninergic neurons have been analyzed (Gillette, 2006; Flavell et al., 2013). In other, more complex animals, e.g. mice, the role of serotonin-releasing neurons has been investigated as an entire "serotonin system" (Dunbar et al., 2010; Fox and Lowry, 2013; Haller, 2013). Here, an approach will be described to disentangle a putative role of distinct serotonergic neurons within the entirety of all serotonin-releasing neurons for an animal's behavior. The fruit fly Drosophila, which is positioned between $C$. elegans and mammals in the neuron number and complexity of the nervous system, represents a model organism in which such a circuit dissection might be technically possible.

\subsection{Drosophila melanogaster as a model organism}

Although the Drosophila brain is much simpler than vertebrate brain and contains approximately $10^{5}$ neurons - which is much less than the number of neurons in human $\left(10^{8}\right.$ $10^{10}$ ) - many complex neuronal circuits and behaviors also exist in the flies, like sleep and circadian rhythms (Hendricks et al., 2000; Tataroglu and Emery, 2014), learning and memory (Qui and Davis, 1993; Fiala, 2007), aggression (Asahina et al., 2014), or addiction (Peru Y Colón de Portugal et al., 2013). At the level of neurophysiology, flies and vertebrates share many similarities, e.g., with respect to neuromodulators and neurotransmitters, the overall architecture of neurons and synapses, or the molecular mechanisms of synaptic plasticity. 
The variety of genetic tools available for Drosophila research provides the possibility to design experimental approaches which are difficult or impossible in other organisms. For example, by using P-element based transgenesis (Rubin and Spradling, 1982), PhiC31 integrase-mediated transgenesis (Groth et al., 2004), binary expression systems and site-directed recombination systems, it is possible to target and manipulate subset of neurons and to study the neuronal anatomy and neuronal function of individual cells and of neuronal circuits (reviewed by Venken et al., 2011). Since genetic techniques to target and manipulate subsets of neurons have been used in this thesis, the genetic approaches available will be explained in the following sections.

\subsubsection{Ectopic expression of transgenes}

\subsubsection{Temporal gene expression using a heat-shock promoter}

By expressing a transgene under the control of a heat-shock promoter, the timing and the level of gene expression can be manipulated. The expression can be induced by applying a heatshock at different developmental stages. In addition, by altering the intensity of the heat-shock in terms of temperature increase, the level of the expression can be controlled to some degree. However, gene expression is ubiquitous and not restricted to specific tissues or cells (Lindquist, 1986; Phelps and Brand, 1998; McGuire et al., 2004a).

\subsubsection{Spatial gene expression}

By fusing the gene of interest downstream of a specific and characterized promoter, a spatially restricted expression can be achieved. The usage of binary expression systems in Drosophila facilitates such an artificial expression of different genes in a spatially restricted pattern. The principle of binary expression systems relies on a transcriptional activator that binds to specific sites on the DNA and induces or enhances the transcription of a downstream gene. By expressing the transcriptional activator under control of a specific promoter, the gene downstream of the transcriptional activator binding site is expressed in a tissue- or cell-specific pattern. 


\section{The UAS-Gal4 binary expression system}

The Gal4 protein is a yeast transcription activator (transactivator) which regulates the expression of galactose-inducible genes at the transcriptional level by binding to Gal4 binding sites called "upstream activation sequence" (UAS). In the absence of the Gal4 protein, the genes downstream of the UAS are not expressed (Laughon et al., 1984). This system is adopted from yeast and used widely as the binary expression system in Drosophila (Brand and Perrimon, 1993; Fischer et al., 1988). The promoter/enhancer-Gal4 transactivator (driver) and UAS:transgene (target/responder) are integrated into the genome of different fly lines. Therefore, by crossing specific UAS and Gal4 fly lines, the responder is expressed in the progeny (Figure $1.2 \mathrm{~A}$ ). The expression pattern of the Gal4 driver lines can be visualized by crossing them to appropriate reporter lines (e.g., UAS:fluorescent proteins). A large number of Gal4 drivers and UAS lines are available in different stock centers (e.g., the Bloomington Drosophila stock center at Indiana University). The efficiency of this system has been further improved by optimizing the expression of Gal4, e.g., by increasing the mRNA stability by replacing a hsp70 transcriptional terminator with a SV40 virus transcriptional terminator, by increasing Gal4 expression levels by replacing the activation domain with virus (VP16) or human (P65) activation domains, by increasing the number of UAS sites, and by adding 5'-UTR and 3'-UTR elements to the UAS constructs (Pfeiffer et al., 2010).

\section{The LexAop-LexA binary expression system}

In this system, the LexA protein as a bacterial transcription activator controls the gene expression by binding to LexA operator (LexAop) sequences (Brent and Ptashne, 1985). This system is used in Drosophila as second binary expression system (Figure 1.2 B). Modified LexA proteins (with the C-terminal activation domain of Gal4 or VP16 added to LexA) can drive expression of the transgenes downstream of the LexAop sites in Drosophila (Szüts and Bienz, 2000; Lai and Lee, 2006). Recently, the P65 activation domain and a nuclear localization domain were added to the LexA protein to increase expression levels and to reduce leaky expression (Pfeiffer et al., 2010). 
A

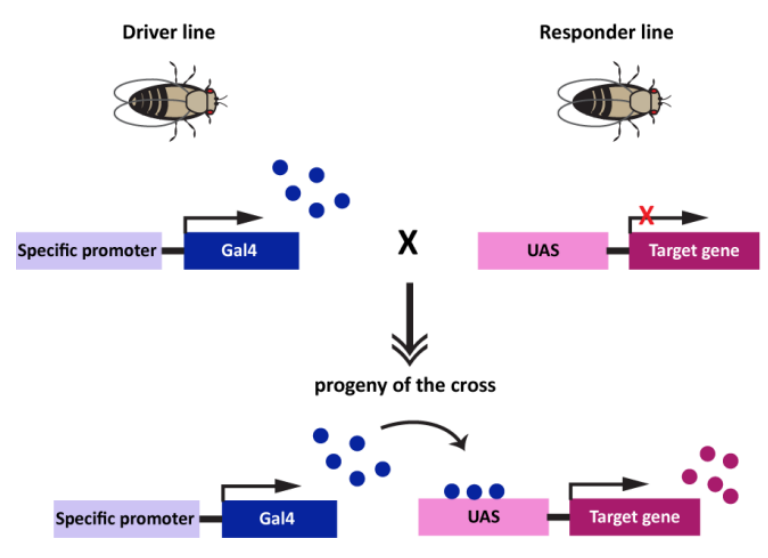

B

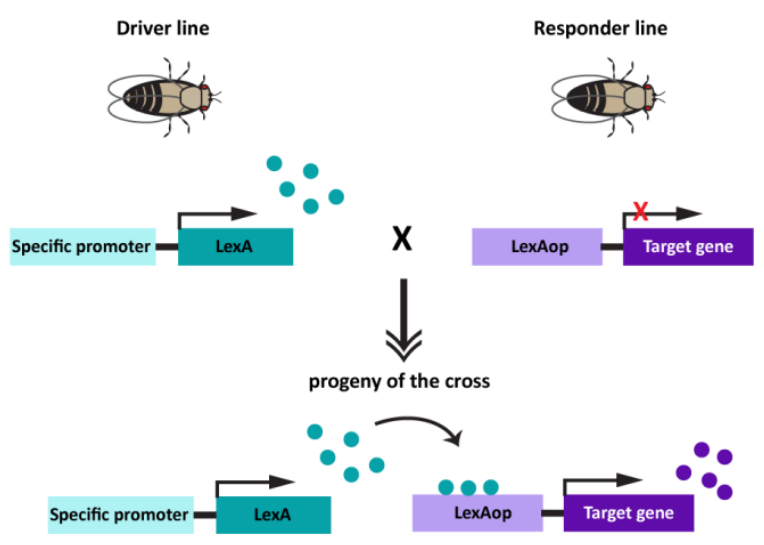

Figure 1.2. Binary expression systems. (A) UAS-Gal4 system. The expression of Gal4 protein as a transactivator is controlled by a tissue/cell specific promoter. Binding of the Gal4 to the upstream activation sequence (UAS) initiates the expression of the gene downstream of the UAS. The promoter/enhancer-Gal4 (driver) and UAS:transgene (responder) are carried in different fly lines. Therefore, by crossing specific UAS and Gal4 parental lines, the responder is expressed in the progeny. Modified from Brand and Perrimon, 1993. (B) LexAop-LexA system. The expression of LexA protein as a transactivator is controlled by a tissue/cell specific promoter. Binding of the LexA to LexA operator (LexAop), the gene downstream of the LexAop is expressed in spatially restricted pattern.

\subsubsection{Spatiotemporal gene expression}

In the yeast, Gal4 activity can be inhibited by the negatively regulating protein Gal80 in the absence of galactose (Laughon et al., 1984). The Gal80 protein also suppresses the Gal4 activity in Drosophila (Lee and Luo, 1999). Using a mutated, temperature sensitive Gal80 protein $\left(G a l 80^{\text {ts }}\right)$, spatiotemporal gene expression can be achieved (McGuire et al., 2004b). The Gal80 ${ }^{\text {ts }}$ suppresses Gal4-induced expression at $19^{\circ} \mathrm{C}$. However, at $30^{\circ} \mathrm{C}$ the Gal $80^{\text {ts }}$ is inactivated and Gal4 induces the expression of the transgene downstream of the UAS site (Figure 1.3). Therefore, by combining the UAS-Gal4 system and the Gal80 ${ }^{\text {ts }}$ (TARGET system), the gene of interest can be expressed in specific cells at a specific time (McGuire et al., 2004b). 

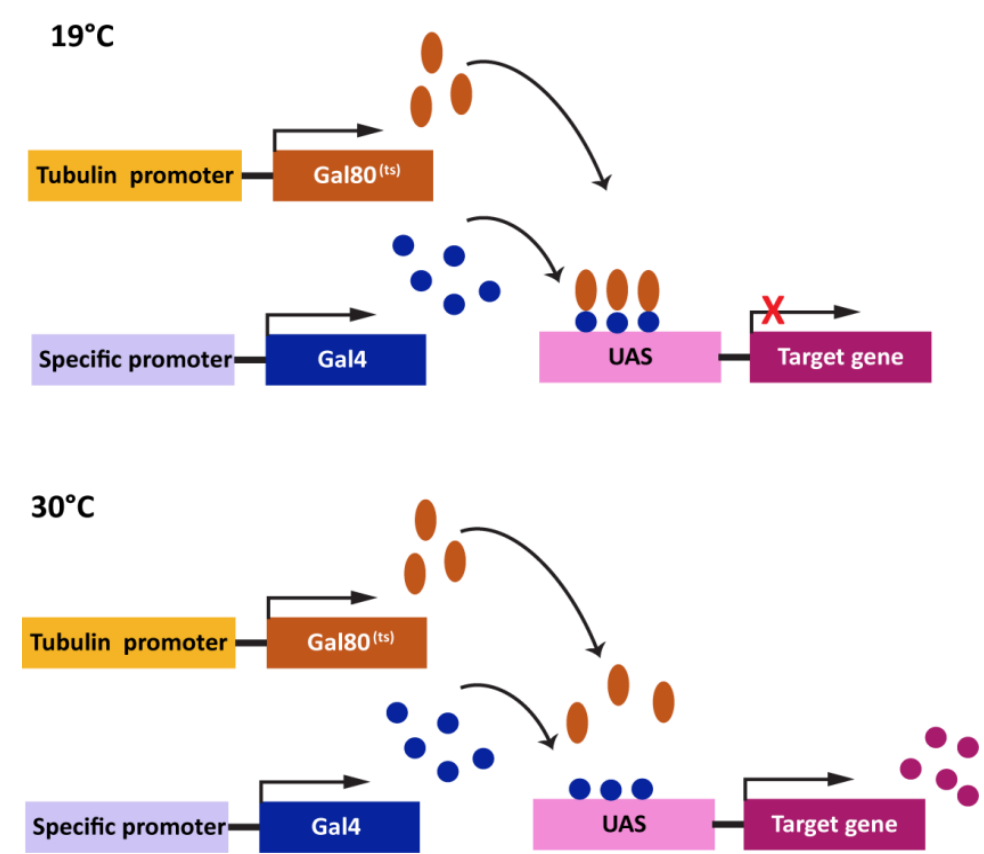

Figure 1.3. TARGET system. Using the temperature sensitive Gal80 protein ( Gal $80^{\text {ts }}$ ), the Gal4-induced gene expression can be inhibited at the permissive temperature $\left(19^{\circ} \mathrm{C}\right)$. At the restrictive temperature $\left(30^{\circ} \mathrm{C}\right)$, the inhibitory effect of Gal80ts is impaired and Gal4 activates the expression of the transgene downstream of the UAS site. Modified from McGuire et al., 2004b.

In the LexAop-LexA system, the LexA fused to the Gal4 activating domain (LexA::GAD) is sensitive to the Gal80 system (Lai or Lee, 2006). Therefore, the spatiotemporal expression of the transgene can be likewise controlled by combining the LexAop-LexA system with Gal80 ${ }^{\text {ts }}$ (Figure 1.4).

A
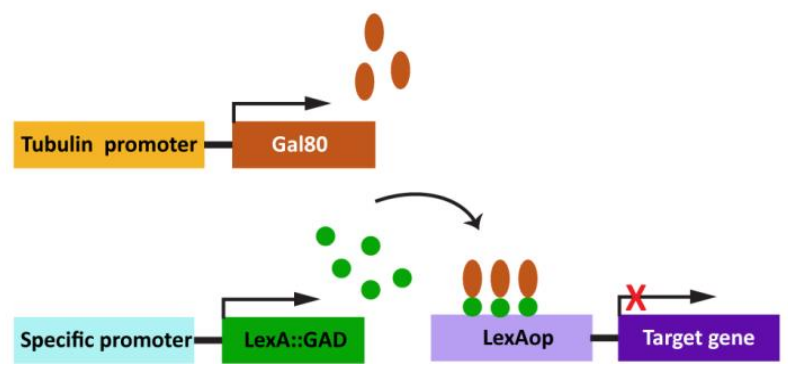

B
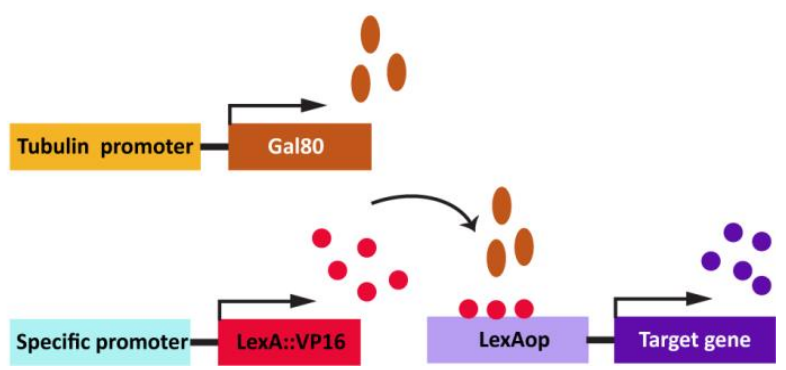

Figure 1.4. Gal80-mediated, inhibitory effect on the LexAop-LexA system. (A) The LexA fused to the Gal4 activating domain (LexA::GAD) is sensitive to Gal80. The expression of the transgene downstream of the LexAop can be inhibited in the presence of Gal80. (B) The LexA fused to the virus activation domain (LexA::VP16), however, is not sensitive to the Gal80. Modified from Lai and Lee, 2006. 


\subsubsection{FLP-FRT -mediated site-directed recombination}

The flippase recombinase (FLP) induces a site-directed recombination of DNA sandwiched between recombination target sites (FRTs). This system was adopted from yeast to induce site directed recombination in Drosophila (Golic and Lindquist, 1989). FLP catalyzes the recombination by inversion of the DNA sequence between the two inverted FRT sites. However, in the case of two direct FRT sites, the excision of the FRT cassette (one FRT site and the DNA sequence in between) is induced by FLP (Figure 1.5). The FLP-FRT system is widely used in Drosophila for stochastic, random expression of a transgene, for intersectional gene expression strategies and also for generating genetic mosaics using the MARCM (Mosaic Analysis with a Repressible Cell Marker) technique (reviewed by Venken et al., 2011).

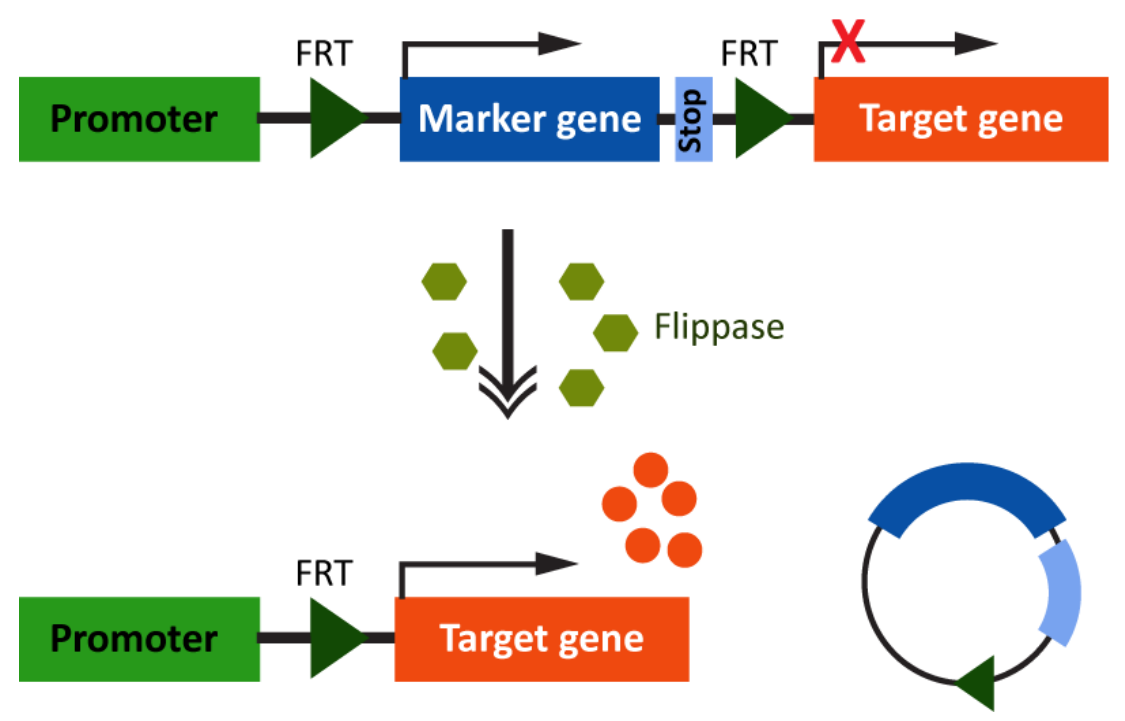

Figure 1.5. The FLP-FRT system. The transcription stop codon positioned in the FRT cassette suppresses the expression of the target gene downstream of the cassette. In the presence of a flippase recombinase (FLP), the site directed recombination at its recombination target (FRTs) leads to the excision of the transcription stop flanked by the FRT sites and the expression of the target gene can be regulated by the promoter. Modified from Golic and Lindquist, 1989. 


\subsubsection{Generating random expression patterns}

By expressing FLP under the control of a heat-shock promoter (hs-FLP), a stochastic expression of the transgene in random cells within the tissue of interest can be obtained. Alteration in the time or temperature of the heat-shocking leads to different FLP expression levels.

Several alternative procedures for achieving a stochastic expression across cells can be designed based on the experiment's requirements. For example, the Gal80 expression pattern and its inhibitory effect on UAS-Gal4 gene expression can be manipulated by using a tub:FRTGal80-FRT-stop fly line. In this fly line, the Gal80 protein is expressed ubiquitously under the control of tubulin promoter (tub) and prevents the Gal4 protein from activating the expression of the transgene downstream of the UAS sequences (Figure 1.6 A). The FLP expression, which is induced by a heat-shock in random subset of the cells using hs-FLP, leads to the excision of Gal80 and expression of UAS:transgene under the control of Gal4 driver line in the FLP positive cells (Figure 1.6 B). Thereby, the transgene can be expressed just in a random subset of the cells within the entirety of all Gal4 expressing cells (Bohm et al., 2010).

An alternative approach makes use of a tub:FRT-stop-FRT-Gal80 fly line that enables one to express a transgene in most of the Gal4 expressing cells and to suppress its expression in other cells. In these flies carrying the tub:FRT-stop-FRT-Gal80 construct, the hs-FLP, the cell typespecific Gal4 gene and UAS:transgene construct, the Gal80 protein cannot be expressed under the control of the tubulin promoter due to the existence of a transcription stop codon upstream of the Gal80. Thereby, the UAS:transgene is expressed under the control of the Gal4 activity (Figure 1.6 C). However, the heat-shocked induced expression of FLP in few, random cells leads to an excision of the FRT stop cassette which results in the expression of the Gal80 (Bohm et al., 2010). Therefore, the expression of the transgene can be repressed in FLP positive cells within the entire Gal4 expression pattern (Figure $1.6 \mathrm{D}$ ). 
A

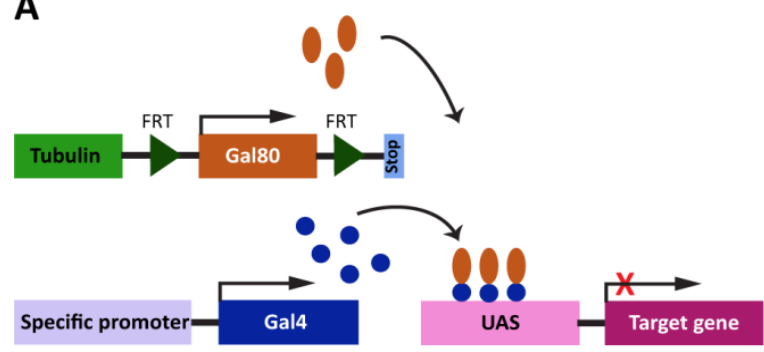

C
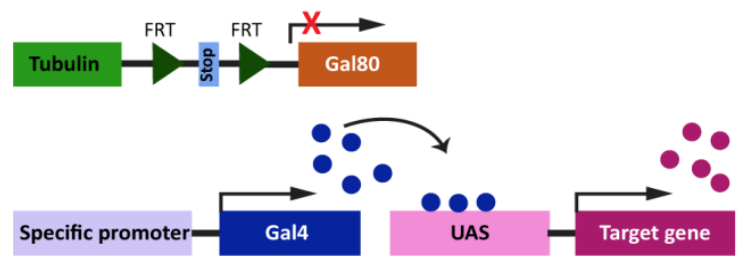

B

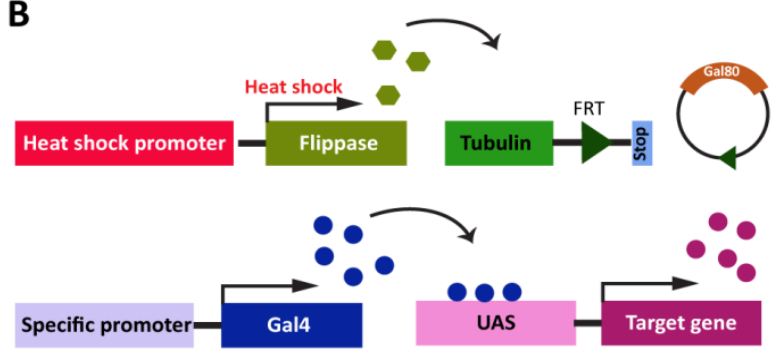

D

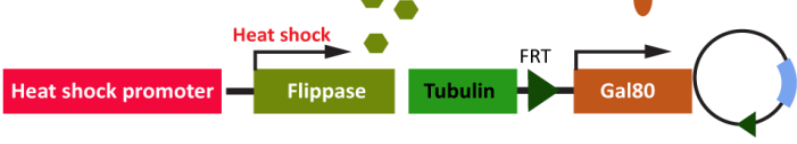

Specific promoter

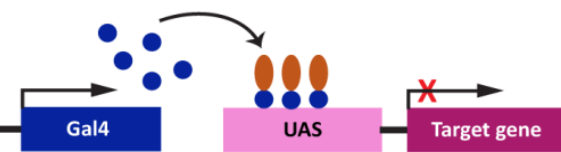

Figure 1.6. Stochastic transgene expression. (A) Tubulin driven Gal80 flanked by FRT sites prevents the expression of the UAS:transgene by inhibiting the Gal4 activity. (B) Heat-shock induced FLP expression in random subset of the cells leads to site directed recombination in FRT sites and excision of Gal80. Therefore, the UAS:transgene can be expressed by Gal4 activity in FLP positive cells. (C) In the tub:FRT-stop-FRT-Gal80 fly line, the Gal80 cannot be expressed under the control of tubulin promoter due to the existence of the transcription stop upstream of the Gal80. Therefore, Gal4 activates the expression of the target gene downstream of the UAS sequence. (D) The expression of the FLP in the random subset of cells under the control of heat-shock promoter leads to excision of transcription stop and expression of the Gal80 protein. Therefore, the expression of the transgene in those few random FLP positive cells is suppressed.

\subsubsection{Intersectional strategies to restrict gene expression patterns}

For so-called "intersectional" gene expression strategies for the expression of a transgene, different techniques like the Gal4-Gal80 system, the split Gal4 system that combines two Gal4 driver lines, and also the FLP-FRT system, are applicable (reviewed by Venken et al., 2011).

In comparison to a stochastic expression strategy, in the FLP-FRT approach for intersectional gene expression, the FLP expression is controlled by a site specific promoter instead of a heatshock promoter. For example, in flies carrying a UAS:FRT-stop-FRT-transgene construct, a promoter A-FLP construct and a promoter B-Gal4 construct, the transgene expression is restricted to overlapping subsets of neurons of promoter $A$ and promoter $B$ (Figure 1.7). In this way, the transgene downstream of the FRT stop cassette can be constantly expressed in the subset of the cells that both show FLP and Gal4 expression. 


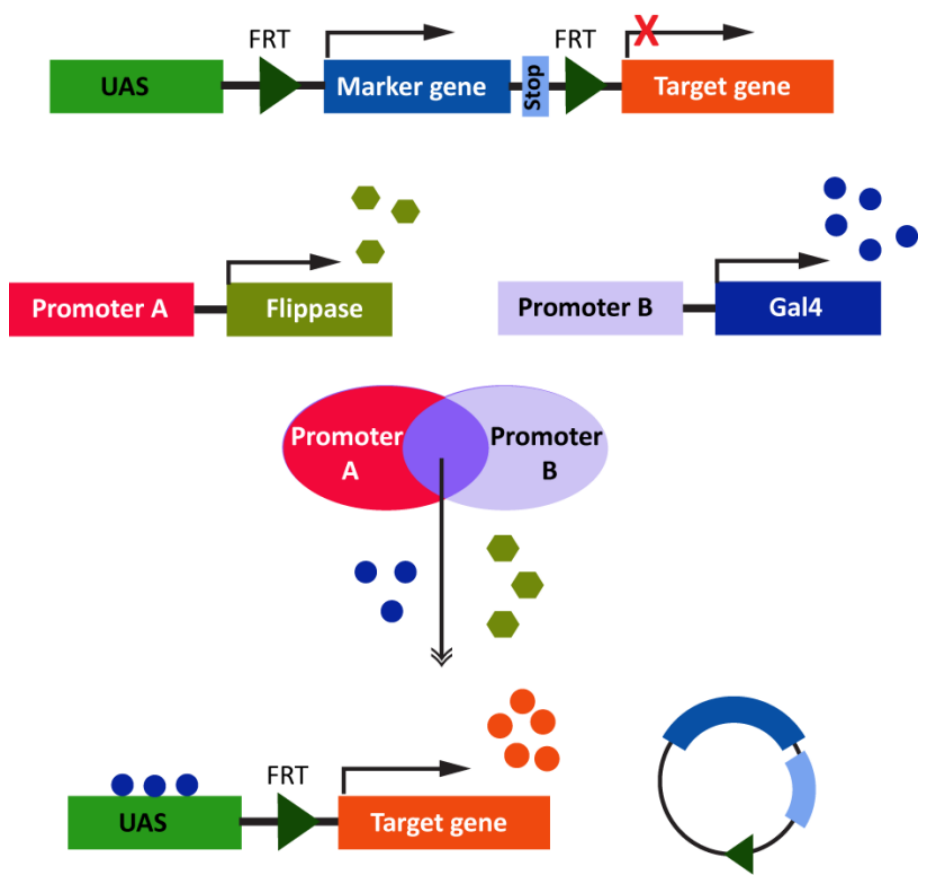

Figure 1.7. "Intersectional" gene expression. By combining the UAS-Gal4 and the FLP-FRT system, the intersectional expression of a transgene in few cells can be achieved. The transgene downstream of the FRT Stop cassette can be expressed under the control of the overlapping domain of the two promoters (promoter A and B) in the cells expressing both FLP and Gal4 proteins.

In the following it will be described how transgene expression can be used to manipulate neuronal activity or synaptic transmission.

\subsubsection{Manipulation of neuronal activity}

By expressing ion channels or toxins in a defined population of neurons (using the gene expression techniques mentioned above), a manipulation of neuronal activity is possible. A variety of possibilities exist to activate or to silence single neurons or populations of neurons. Thereby, their roles in neuronal circuits or regulating specific behaviors can be investigated.

A most direct approach makes use of the expression of apoptotic genes like reaper, hid or grim (Wing et al., 1998), which induce cell death in a specific population of the cells. Blocking synaptic transmission can be achieved by the modification of vesicle release. Tetanus toxin (UAS:TNT), for example, disrupts neuronal synaptobrevin (nSyb) in synaptic vesicles, which results in a silencing of the neuronal transmission by inhibiting the neurotransmitter release (Sweeney et al., 1995). The continuous expression of TNT during development is a disadvantage 
of using this toxin for silencing neuronal activity, which can to a certain degree be overcome by temporally induced gene expression. Increasing the potassium conductance by expressing ion channels prevents a depolarization of the neurons. UAS:Kir2.1 (inward rectifying potassium channel) and UAS:dORK (two-pore leak potassium channel) are two examples of ion channels which are widely used to inhibit neuronal excitability (Baines et al., 2001; Nitabach et al., 2002). Another way to silence neuronal transmission in a temporally controlled way is the usage of the temperature sensitive shibire protein $\left(\mathrm{Shi}^{(\mathrm{ts})}\right)$. The shibire gene encodes the protein shibire, the Drosophila homologue of the vertebrate protein dynamin, which is involved in the process of endocytosis and which is essential for synaptic vesicle recycling. The mutated, temperaturesensitive variant $\mathrm{Shi}^{\left({ }^{(\mathrm{ts})}\right.}$ becomes non-functional at the restrictive temperature $\left(>29^{\circ} \mathrm{C}\right)$, thereby inhibiting the synaptic vesicle recycling reversibly. However, synaptic transmission remains intact in the permissive temperature $\left(19-22^{\circ} \mathrm{C}\right)$ (Kitamoto, 2001).

To temporally increase neuronal activity, the light-activated cation channel, channelrhodopsin2 (ChR2) can be used. Blue light stimulation of 450-490 nm wavelength opens the cation channel (Nagel et al., 2005), leading to a depolarization of the neuron that expresses it. In addition, a thermogenetic approach is a widely used method for remote activation of neurons in Drosophila. By expressing the temperature-sensitive cation channel, dTRPA1, in a specific population of neurons, their neuronal activity can be triggered by increasing the temperature (Hamada et al., 2008; Pulver et al., 2009).

In mammals, several subsets of the endogenously expressed transient receptor potential (TRP) channels, including four TRPVs, TRPM8 and TRPA1, act as thermo-sensors. Each thermoTRP channel is activated by a distinct temperature (from noxious cold to noxious hot). These channels typically consist of intracellular amino and carboxyl termini with six transmembrane domains and a pore region between domain 5 and 6 (Patapoutian et al., 2003). TRPV1 and TRPV2 channels are activated by noxious hot temperatures $\left(\geq 42^{\circ}\right.$ and $\left.\geq 52^{\circ} \mathrm{C}\right)$, TRPV 3 and TRPV 4 by moderately warm temperatures $\left(233^{\circ} \mathrm{C}\right.$ and $27^{\circ} \mathrm{C}$ to $42^{\circ} \mathrm{C}$ ), TRPM 8 by moderately cool temperatures $\left(\leq 25^{\circ} \mathrm{C}\right)$ and TRPA1 by noxious cold temperatures $\left(\leq 17^{\circ} \mathrm{C}\right)$ (reviewed by Dhaka et al., 2006). For mammalian TRPV and TRPM families, no orthologue in invertebrates exists. However, mammalian TRPA1 (the only member of this TRP subfamily) has an orthologue in Drosophila (dTRPA1). The TRPA subfamily is required for proper thermo sensation in Drosophila. In contrast to the mammal orthologue, dTRPA1 channels respond to warm temperatures rather than to cold temperatures (Viswanath et al., 2003). Three thermoTRP 
channels in Drosophila (from the TRPA subfamily) have been identified, ITRPA1, pyrexia and painless (Viswanath et al., 2003; Lee et al., 2005; Tracey et al., 2003). dTRPA1 responds to temperatures between $25^{\circ} \mathrm{C}$ and $29^{\circ} \mathrm{C}$. Pyrexia is activated by temperatures higher than $35^{\circ} \mathrm{C}$, and painless activation is induced at a temperature higher than $42^{\circ} \mathrm{C}$. Although the dTRPA1 channel is endogenously expressed in three sets of cells in the brain including lateral cell (LC), Ventral cell (VC) and anterior cell (AC), only the AC neurons have been reported to act as thermo-sensors (Hamada et al., 2008). As a thermogenetic tool for remote heat activation of neurons and to investigate neuronal circuits and behavior, the dTRPA1 channel can be expressed ectopically in different cell population using the UAS-Gal4 system or alternative gene expression strategies (Parisky et al., 2008; Shang et al., 2008; Pulver et al., 2009).

\subsection{Serotoninergic neurons in Drosophila}

\subsubsection{Serotonin biosynthesis, transportation, metabolism and receptors}

The serotonin synthesis pathway starts with the hydroxylation of the amino acid tryptophan by the enzyme tryptophan hydroxylase (TRH). This reaction is the rate-limiting step in serotonin biosynthesis. In Drosophila, two types of enzymes, tryptophan hydroxylase (DTRHn) and phenylalanine hydroxylase (DTPHu) produce serotonin in neuronal and non-neuronal tissues, respectively. In the second step of serotonin biosynthesis, 5-hydroxytryptophan is decarboxylated by 3, 4-dihydroxyphenylalanine decarboxylase (DOPA decarboxylase or DDC) (reviewed by Blenau and Thamm, 2011; Figure 1.8). The biosynthesis of serotonin is blocked by p-chlorophenylalanine (PCPA) (Figure 1.8). PCPA is an inhibitor of TRH and it has been used in many experiments to investigate the effect of serotonin depletion on different behaviors in various organisms (Koe and Wiessman, 1966; Banerjee et al., 2004; Dasari et al., 2007). 


\title{
Tryptophan
}

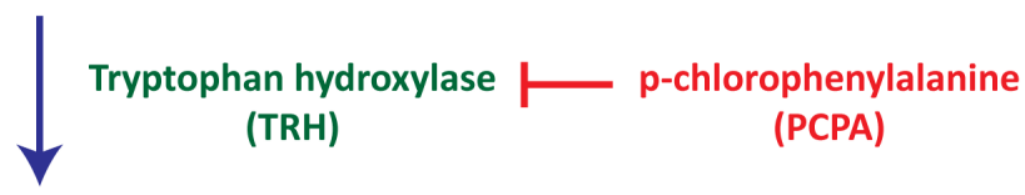

\section{5-Hydroxytryptophan}

\author{
DOPA decarboxylase \\ (DDC)

\section{5-Hydroxytryptamine} \\ (serotonin)
}

Figure 1.8. Serotonin biosynthesis pathway. Tryptophan is hydroxylated by tryptophan hydroxylase (TRH) to 5hydroxytryptophan, which is decarboxylated by DOPA decarboxylase (DDC) to serotonin. p-chlorophenylalanine (PCPA) acts as an inhibitor of TRH and blocks the biosynthesis of serotonin.

Serotonin in Drosophila is transported by a specific serotonin transporter (dSERT). The characterization of dSERT showed that this protein has a high affinity to bind serotonin and it also binds the transport inhibitor cocaine. However, some properties of dSERT are different from human SERT (hSERT). For example in comparison to the hSERT, dSERT does not need $\mathrm{Cl}^{-}$ ions for serotonin transportation and also has a lower sensitivity to antidepressant drugs (like fluoxetine) (reviewed by Blenau et al., 2013). The pan-neuronal expression of the dSERT in fly CNS has shown that only a small number of neurons can import and store serotonin (Park et al., 2006b).

Serotonin and other biogenic amines in mammals are metabolized by the monoamine oxidase (Mao) and catechol-O-methyl transferase (COMT). However, in insects, alternative metabolic pathways involving biogenic amines are $\mathrm{N}$-acetylation by the activity of arylalkylamine $\mathrm{N}$ acetyltransferase (AANAT1 and 2), sugar conjugation and also sulfation of a hydroxyl group (reviewed by Sloley, 2004). Furthermore, ß-alanine conjugation by the Ebony protein is another pathway for the degradation of biogenic amines (Richardt et al., 2003).

In Drosophila, serotonin acts as a neurotransmitter and neuromodulator through five types of G-protein-coupled 5-HT receptors (reviewed by Blenau et al., 2013). All of these receptors are well studied. They are differentially expressed in the distinct brain regions and they can act inhibitory or activating. They do so by influencing secondary messenger systems in the cells. 
The properties and expression pattern of 5-HT receptors in Drosophila are summarized in table 1.1.

Table 1.1. 5-HT receptors in Drosophila

\begin{tabular}{|c|c|c|c|}
\hline $\begin{array}{l}\text { 5-HT } \\
\text { receptor }\end{array}$ & Described by & Properties (references) & $\begin{array}{l}\text { Expression pattern in adult fly } \\
\text { (references) }\end{array}$ \\
\hline 5-HT1A & $\begin{array}{l}\text { Saudou et al., } \\
1992\end{array}$ & $\begin{array}{l}\text { Negatively coupled with } \\
\text { adenylate cyclase, } \\
\text { activate phospholipase } \\
\text { C, orthologous of } \\
\text { mammalian 5-HT1A } \\
\text { (Saudou et al., 1992) }\end{array}$ & $\begin{array}{l}\text { Mushroom bodies, subpopulation of } \\
\text { median neurosecretory cells (IPCs) } \\
\text { (Yuan et al., 2005; Luo et al., 2012) }\end{array}$ \\
\hline $5-\mathrm{HT} 1 \mathrm{~B}$ & $\begin{array}{l}\text { Saudou et al., } \\
1992\end{array}$ & $\begin{array}{l}\text { Negatively coupled with } \\
\text { adenylate cyclase, } \\
\text { activate phospholipase } \\
\text { C, orthologous of } \\
\text { mammalian 5-HT1A } \\
\text { (Saudou et al., 1992) }\end{array}$ & $\begin{array}{l}\text { Mushroom bodies, median } \\
\text { neurosecretory cells, clock neurons like } \\
\text { I-LNvS and s-LNVS, optic lobes, } \\
\text { subesophageal ganglion in } \\
\text { serotoninergic neurons } \\
\text { (Yuan et al., 2006) }\end{array}$ \\
\hline $5-\mathrm{HT} 2 \mathrm{~A}$ & $\begin{array}{l}\text { Colas et al., } \\
1995\end{array}$ & $\begin{array}{l}\text { orthologous of } \\
\text { mammalian 5-HT2, } \\
\text { possible effect on } \\
\text { inositol trisphosphate } \\
\text { stimulation } \\
\text { (Colas et al., 1995) }\end{array}$ & $\begin{array}{l}\text { ellipsoid body, large field R neurons, } \\
\text { cells in protocerebrum including } \\
\text { multipolar neurons around calyces } \\
\text { (Nichols, 2007) }\end{array}$ \\
\hline $5-\mathrm{HT} 2 \mathrm{~B}$ & $\begin{array}{l}\text { Clark et al., } \\
2004\end{array}$ & $\begin{array}{l}\text { Orthologous of } \\
\text { mammalian 5-HT2, } \\
\text { coupled with the Gq } \\
\text { signaling pathway } \\
\text { ( Clark et al., 2004) }\end{array}$ & $\begin{array}{l}\text { synaptic neuropil of the stomatogastric } \\
\text { ganglion and axon terminals of } \\
\text { stomatogastric neurons (modulating the } \\
\text { motor output of stomatogastric), } \\
\text { differentially expressed in male and } \\
\text { female nervous tissue } \\
\text { (Clark et al., 2004; Goldman and } \\
\text { Arbeitman, 2007) }\end{array}$ \\
\hline 5-HT7 & $\begin{array}{l}\text { Witz et al., } \\
1990\end{array}$ & $\begin{array}{l}\text { Activating adenylate } \\
\text { cyclase, orthologous of } \\
\text { mammalian 5-HT7 } \\
\text { (Witz et al., 1990) }\end{array}$ & $\begin{array}{l}\text { All large-field R neurons that innervate } \\
\text { the ellipsoid body, small groups of cells } \\
\text { that tightly cluster with PDF positive LNV } \\
\text { neurons, low expression in upper region } \\
\text { of fan shape body, thoracic and } \\
\text { abdominal neuromeres } \\
\text { (Becnel et al., 2011) }\end{array}$ \\
\hline
\end{tabular}




\subsubsection{The distribution of serotonin-releasing neurons in the Drosophila brain}

Serotoninergic neurons in Drosophila consist of around 84 cell bodies in the larval stage and around 100 cell bodies in the adult stage. Although the number of serotoninergic neurons is a small fraction of the total number of neurons in the fruit fly $\left(10^{5}\right)$, the entire brain is densely innervated by them (Valles and White, 1988). Using antibody staining against serotonin, nine distinct neuronal clusters have been identified by Valles and White (SP1, Sp2, IP, LP1, SE1, SE2, SE3, LP2a and LP2b). Based on this study, the SE1, SE2 and SE3 clusters are located in the subesophageal ganglion, the SP1, SP2, IP clusters in the anterior medial protocerebrum, the LP1 cluster in the lateral protocerebrum adjacent to anterior optic tract and the LP2 $2_{a, b}$ cluster in the ventrolateral protocerebrum adjacent to medulla layer (Valles and White, 1988; Figure 1.9). Sitaraman et al. (2008) identified more clusters and reassigned the names some clusters. The newly described clusters by Sitaraman et al. consist of the anterior lateral protocerebrum (ALP) and anterior medial protocerebrum (AMP). Although the SP1, Sp2, IP, LP1 clusters were described as anterior clusters by Valles and White, recent studies found these clusters to be located in the posterior part of the brain (Sitaraman et al., 2008; Alekseyenko et al., 2010; Pech et al. 2013). Therefore, the SP1, SP2 and IP clusters from Valles and White were reassigned to posterior medial protocerebrum (PMP) cluster and LP1 to posterior lateral protocerebrum (PLP) cluster (Sitaraman et al., 2008; Figure 1.9). Comparable results in the distribution of the serotoninergic cells were obtained by Alekseyenko et al. (2010) and Pech et al. (2013).

A

B

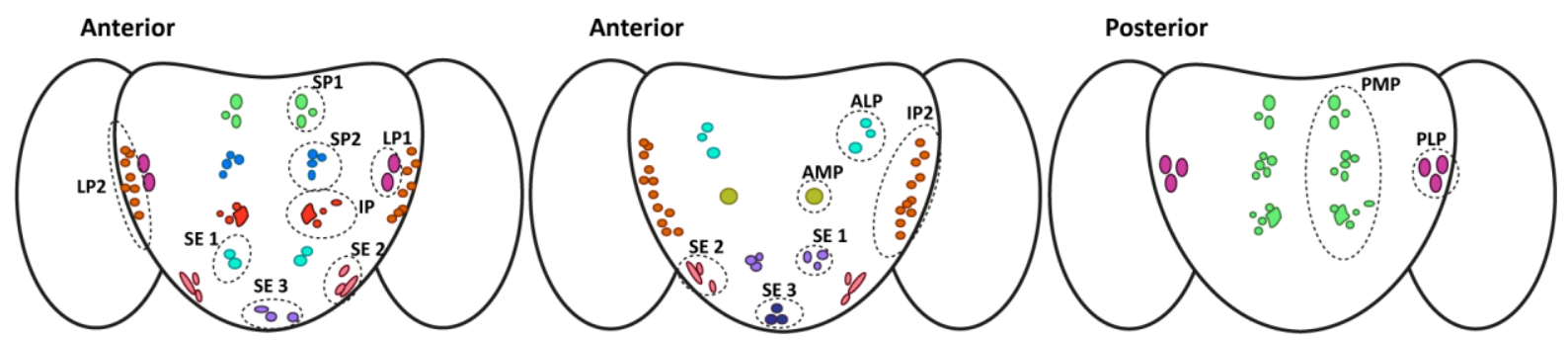

Figure 1.9. Distribution of serotoninergic cell clusters in the adult Drosophila brain according to Valles and White, (1988) and Sitaraman et al., (2008). (A) Schematic illustration of the anterior view of the Drosophila brain and the localization pattern of the serotoninergic clusters in the anterior region (SP1, SP2, SP3, LP1, LP2, SE1, SE2, and SE3) according to the study of Valles and White, (1988). (B) Schematic illustration of the anterior and posterior views of the Drosophila brain and the localization pattern of the serotoninergic clusters in the anterior (ALP, AMP, IP2, SE1, SE2, and SE3) and the posterior (PLP and PMP) regions of the brain according to the study of Sitaraman et al., (2008). 


\subsection{Behavioral roles of serotonin in Drosophila melanogaster}

Several studies on Drosophila have shown a modulatory effect of serotonin on a variety of behaviors.

\section{Sleep and circadian rhythm}

In a study by Yuan et al. (2005), a modulatory effect of serotonin on responses of clock neurons to light has been shown. In this study, the 5-HT1B receptor expressed in clock neurons (LNvs) was found to be involved in this pathway. Elevating the level of serotonin using 5-HTP (a 5-HT precursor) or the drug Prozac (a serotonin reuptake inhibitor) and also the overexpression of the 5-HT1B receptor led to a reduction in the circadian light sensitivity in Drosophila (Yuan et al. 2005).

A regulatory effect of serotonin on sleep through the 5-HT1A receptor has been shown by Yuan et al. (2006). An increase in the serotonin level, using pharmacological (5-HTP) or genetic (UAS:TRH, DDC-Gal4) approaches, promoted sleep in Drosophila. Differential effects of 5-HT1A, 5-HT1B and 5-HT2 receptors on baseline sleep were verified in this study using mutants and RNAi lines. The 5-HT1A mutant showed a reduction in sleep time, but reducing the expression of the 5-HT1B and 5-HT2 receptors did not affect the baseline sleep (Yuan et al. 2006).

The involvement of the 5-HT2A receptor in regulating circadian behavior has been shown by Nichols (2007). In their study, an agonist of the 5-HT2A receptor (DOI) increased the early day activity and eliminated the anticipatory behavior before light onset (Nichols, 2007).

\section{Aggression behavior}

In a study by Dierick and Greenspan (2007), a molecular analysis of a fly line selected for escalated fighting did not reveal any difference in 5-HT-related gene expression. Therefore, the authors have suggested that global changes in the 5-HT level do not play any role in aggression. However, in a study from 2007, the authors examined the direct effect of 5-HT on aggression using a pharmacological approach. In this study, flies were fed with the 5-HT precursor 5-HTP or with a 5-HT synthesis inhibitor (aMTP) to increase or decrease the 5-HT level in the brain, respectively. They found that elevating the serotonin level in the brain caused an increase in fighting frequency, although the reduction of the serotonin level did not have any effect on aggression. They further found by genetic manipulation of the serotonin level using UAS:TRH; 
DDC-Gal4 for increasing it or using UAS:TNT, DDC-Gal4 for decreasing it, that serotonin has a modulatory effect on aggression but is not required (Dierick and Greenspan, 2007).

Using a thermogenetic approach to manipulate serotoninergic neurons, a serotonin-dependent change in the aggression level was claimed by Alekseyenko et al. (2010). Based on this study, activation of the serotoninergic neurons using UAS:dTRPA 1 at $26^{\circ} \mathrm{C}$ led to an escalation of aggression. However, blocking these neurons using UAS: Shi ${ }^{(\mathrm{ts})}$ at $30^{\circ} \mathrm{C}$ abolished the mid and high-level aggression in the flies (Alekseyenko et al., 2010).

\section{Feeding behavior}

Neckameyer et al. found in a study from 2007 that the reduction in the serotonin synthesis using a tyrosine hydroxylase (TRH) mutant led to a reduction in feeding behavior.

The involvement of the 5-HT2A receptor in regulating food intake of Drosophila larvae was shown by Gasque et al. (2013). In this study, uptake of a 5-HT receptor antagonist, metitepine, led to a reduction in food intake in larvae (Gasque et al., 2013).

\section{Locomotion}

The reduction in the serotonin synthesis using a tyrosine hydroxylase (TRH) mutant causes a reduction in locomotion in adult Drosophila, but has no effect on larval locomotion (Neckameyer et al., 2007).

However a recent study of Drosophila larvae showed an inhibitory effect of serotonin on locomotion. Increasing the 5-HT level using transporter inhibitor drugs (fluoxetine and MDMA) led to reduction in the locomotion and the decreasing the 5-HT level by mutation in DDC enzyme caused a significant increase in the locomotion in larvae (Silva et al., 2014).

\section{Courtship behavior}

An involvement of the 5-HT7 receptor in courtship behavior was shown by Becnel et al. (2011) using pharmacological and genetic approaches. Flies with reduced 5-HT7 receptor responsiveness (using the 5-HT7 antagonist SB258719 or 5-HT7 RNAi) showed significantly decreased courtship behavior (Becnel et al., 2011). 


\section{Learning and memory}

A role of serotonin in place memory formation was shown by Sitaraman et al., (2008). In this study, a correlation between a reduction in the serotonin level, using pharmacological (reduction of the serotonin synthesis by the drug aMTP) and genetic (blocking signal transduction in serotoninergic neurons using UAS:TNT, DDC-Gal4, TH-Gal80 flies) approaches, and a reduction in place memory in a heat box learning paradigm was shown (Sitaraman et al., 2008).

A modulatory effect of serotoninergic neurons innervating the mushroom bodies on the formation of anesthesia-resistant memory (ARM) in Drosophila was shown by Lee et al. (2011). In that study, serotonin was reduced using the PCPA drug (tyrosine hydroxylase inhibitor). Furthermore, it was shown that serotonin affects ARM via the 5-HT1A receptor expressed in the mushroom bodies (Lee et al., 2011).

In another study by Sitaraman et al. (2012), it was shown that the serotoninergic system was also required for normal appetitive, olfactory memory formation. In that study, blocking synaptic transmission by expressing UAS:TNT or UAS:Kir 2.1 under the control of the TRH-Gal4 driver line led to impaired appetitive memory (Sitaraman et al., 2012).

All of these studies indicate that serotonin plays a role in modulating a variety of behaviors. It remains, however, unclear, whether distinct serotoninergic neurons have particular functions for the diverse types of behavior, or whether the differential behavioral readouts reflect effects of a common "serotonin system". In this thesis, the role of serotonin in arousal has been investigated. Therefore, the current knowledge about arousal in Drosophila will be summarized. 


\subsection{Arousal in Drosophila}

In general, the "arousal level" can be categorized into endogenous and exogenous arousal. In the endogenous arousal, sleep and wakefulness are regarded to represent the extreme ends. The exogenous arousal can be described as responsiveness to environmental stimuli like startle or mechanical stimuli (Van Swindern and Andretic, 2003; Lebestky et al., 2009; reviewed by Van Swindern and Andretic, 2011).

In Drosophila, a modulatory effect of different biogenic amines on different states of arousal has been shown. For example, a cluster of octopaminergic cells (ASM cluster) acts as wakepromoting neurons in Drosophila (Crocker et al., 2010). Furthermore, dopamine is regarded to be an important neuromodulator involved in different types of arousal. In several studies, a negative effect of dopamine on sleep was reported. For instance, the elevation of the overall dopamine level in fumin flies (a mutant for the dopamine transporter DAT) leads to less sleep and to hyper-responsiveness to mechanical stimuli (Kume et al., 2005). However, an increased dopamine level using methamphetamine causes hyperactivity and less sleep, but it reduces responsiveness (Andretic et al., 2005). Therefore the effect of dopamine on arousal is not simple and linear. In another study, the effect of dopamine through the DopR1 receptor on both endogenous arousal (sleep-wake) and exogenous arousal (air-puff response) was shown by Lebestky et al. (2009). Here, on the one hand, DopR1 mutant flies reveal more responsiveness to an air-puff stimulus, which indicates an inhibitory effect of dopamine on exogenous arousal. On the other hand, these mutant flies were found to sleep more. Therefore, dopamine has a regulatory effect on different types of arousal through distinct pathways, and a differential involvement of DopR1 in different arousal circuits can be concluded (Lebestky et al., 2009).

The regulatory effect of serotonin on circadian rhythm and sleep through different serotonin receptors was shown in several studies (Yuan et al., 2005; Yuan et al., 2006; Nichols, 2007). In addition to sleep, serotonin regulates broad range of behaviors like feeding, locomotion, aggression, learning and memory and courtship in Drosophila (Neckameyer et al., 2007; Silva et al., 2014; Alekseyenko et al., 2010; Sitaraman et al., 2008; Becnel et al., 2011). However, the effect of serotonin on these behaviors is a modulatory one, and serotonin does not seem to have the principle role in driving them (Lucki, 1998). An optimal arousal state is, however, necessary for an optimized behavioral response and causes the motivation for the performance 
(Hebb, 1955). Serotonin might perhaps be involved upstream of behavior initiation by setting the arousal threshold.

\subsection{Aim of this study}

This study uses Drosophila as a model and the serotonergic system as a study case to ask if the arousal system is based on a single and uni-dimentional physiological factor controlling and potentiating every behavior (Hebb, 1955) or if multiple and task-specific arousal systems regulate distinct behaviors (Robbins, 1997; reviewed by Berlucchi, 1997; reviewed by Jing et al. 2009). It further asks whether the neuronal mechanism underlying arousal is a global function of a neurotransmitter in the brain, or whether it is localized to the function of specific circuits.

To address the first question, a new transgenes and transgenic flies were generated that allows for both the thermogenetic activation of the serotonergic neurons and their visualization (i.e. identification). A behavioral assay was established to use the locomotor activity of individual flies as readout of the animal's arousal state. Locomotor activity is a commonly used indicator for measuring arousal level in Drosophila (Shaw et al., 2000). First, the effect of an activation of the serotonergic system on the activity was, therefore, to be distinguished from motor deficits. Arousal is furthermore defined by an alertness to sensory stimuli of all sorts (Pfaff, 2005a). So the behavioral response to differential external stimuli was to be tested in the second step. Further, the task-specifity was to be addressed by testing the behavioral response to punitive mechanical forces on the one hand and an appetitive food stimulus on the other hand. The third criterion that defines arousal is emotional reactivity (Pfaff, 2005a). This cannot be tested in Drosophila. Further, it was to be asked whether an activation of the serotoninergic system also influences the extreme ends of endogenous arousal, sleep and wake states. Third, it was to be asked whether a transient silencing of the serotonergic system would have the opposite effect on the animal's behavior, i.e. whether the serotonergic system is not only sufficient, but also necessary for a putative effect on arousal.

To address the question of whether potential neuronal mechanism of setting arousal levels can be localized to certain circuits, a new transgene construct was created that allows for the stochastic thermogenetic activation and visualization of individual serotonergic neurons. In combination with a single fly behavioral assay, this enables to reveal potential correlations 
between number and/or subtypes of activated serotonergic neurons and the arousal phenotype. Finally, the indications obtained from this experiment were to be confirmed. A transgenic fly was generated that allows for the activation of defined individual neurons in an intersectional approach, which thereby allows for testing the sufficiency of identifiable neurons in setting the animals' arousal threshold. 


\section{Materials and methods}

\subsection{Materials}

\subsubsection{Drosophila melanogaster strains}

\begin{tabular}{|c|c|c|}
\hline Genotype & Description & Source \\
\hline Canton-S (CS) & Wild-type strain & Bloomington (1) \\
\hline$w^{-} ;$TRH-Gal4; + (1) & $\begin{array}{l}\text { Gal4 expression mainly in } \\
\text { serotoninergic neurons }\end{array}$ & $\begin{array}{l}\text { Provided by Dr. Jaeseob Kim } \\
\text { KAIST, Korea } \\
\text { (Park et al., 2006a) }\end{array}$ \\
\hline$y^{+}, w^{-} ;$TRH-Gal4; + (2) & $\begin{array}{l}\text { Gal4 expression mainly in } \\
\text { serotoninergic neurons }\end{array}$ & $\begin{array}{l}\text { Provided by Dr. Serge } \\
\text { Birman, ESPCI Paris Tech, } \\
\text { Paris, France } \\
\text { (Sitaraman et al., 2012) }\end{array}$ \\
\hline$w^{-} ;$TRH-Gal4; TRH-Gal4 (3) & $\begin{array}{l}\text { Gal4 expression mainly in } \\
\text { serotoninergic neurons }\end{array}$ & $\begin{array}{l}\text { Provided by Dr. Edward } \\
\text { Kravitz, Harvard medical } \\
\text { school, Boston, MA } \\
\text { (Alekseyenko et al., 2010) }\end{array}$ \\
\hline$w^{-} ;$actin-Gal4/cyo; + & Ubiquitous expression of Gal4 & $\begin{array}{l}\text { Provided by Prof. Erich } \\
\text { Buchner, Würzburg } \\
\text { university, Würzburg, } \\
\text { Germany } \\
\text { (Funk et al., 2004) }\end{array}$ \\
\hline$w^{-} ;+;$D42-Gal4 & $\begin{array}{l}\text { Gal4 Expression in several } \\
\text { motor neurons }\end{array}$ & $\begin{array}{l}\text { Bloomington (8816) } \\
\text { (Parkes et al., 1998) }\end{array}$ \\
\hline $\begin{array}{l}\text { w',5xUAS:dTRPA1-mCherry; +; } \\
+\end{array}$ & $\begin{array}{l}\text { Expression of dTRPA1-mCherry } \\
\text { under UAS control }\end{array}$ & $\begin{array}{l}\text { Self-generated } \\
\text { (This thesis) }\end{array}$ \\
\hline
\end{tabular}




\begin{tabular}{|c|c|c|}
\hline$w^{-} ;+; 20 x U A S: S h i^{t s}$ & $\begin{array}{l}\text { Expression of } \mathrm{Shi}^{\mathrm{ts}} \text { under UAS } \\
\text { control }\end{array}$ & $\begin{array}{l}\text { Provided by Dr. Hiromu } \\
\text { Tanimoto, Tohoku university, } \\
\text { Sendai, Japan } \\
\text { (Kitamoto, 2001) }\end{array}$ \\
\hline$w^{-} ;$UAS:cameleon 2.1; + & $\begin{array}{l}\text { Expression of cameleon } 2.1 \\
\text { under UAS control }\end{array}$ & (Fiala et al., 2002) \\
\hline$w^{-} ;+; 10 x U A S: m C D 8-G F P$ & $\begin{array}{l}\text { Expression of mCD8-GFP under } \\
\text { UAS control }\end{array}$ & $\begin{array}{l}\text { Bloomington (32185) } \\
\text { (Pfeiffer et al., 2010) }\end{array}$ \\
\hline$y^{+}, w^{-} ;$Tsh-Gal80/cyo; + & $\begin{array}{l}\text { Gal80 expression in thoracic } \\
\text { ganglion }\end{array}$ & $\begin{array}{l}\text { Provided by Dr. Andreas } \\
\text { Thum, Konstanz university, } \\
\text { Konstanz Germany } \\
\text { (Clyne et al., 2008) }\end{array}$ \\
\hline$y^{+}, w^{-}, h s-F L P ;+;+$ & $\begin{array}{l}\text { Expression of flippase under } \\
\text { the control of heat-shock } \\
\text { promoter. }\end{array}$ & $\begin{array}{l}\text { Provided by Dr. Gary Struhl, } \\
\text { Columbia university, New } \\
\text { York, NY } \\
\text { (Basler and Struhl, 1994) }\end{array}$ \\
\hline $\begin{array}{l}w^{-} ; \text {UAS:FRT-CD2-stop-FRT- } \\
\text { mCherry-dTRPA1/gla; + }\end{array}$ & $\begin{array}{l}\text { Expression of mCherry-dTRPA1 } \\
\text { under control of flippase. }\end{array}$ & $\begin{array}{l}\text { Self-generated } \\
\text { (This thesis) }\end{array}$ \\
\hline $\begin{array}{l}\text { w, actin-FRT-Stop-FRT-Gal4; } \\
\text { UAS:GFP; + }\end{array}$ & $\begin{array}{l}\text { Expression of GFP under } \\
\text { control of flippase and actin } \\
\text { promoter. }\end{array}$ & $\begin{array}{l}\text { Provided by Dr. Bing Zhang, } \\
\text { Cornell university, Ithaca, NY } \\
\text { (Pignoni and Zipursky, 1997) }\end{array}$ \\
\hline $\begin{array}{l}y^{+}, w^{-} ;+; \text {UAS:FRT-Stop-FRT- } \\
m C D 8: G F P(2 x) / T M 6\end{array}$ & $\begin{array}{l}\text { Expression of GFP under } \\
\text { control of flippase. }\end{array}$ & $\begin{array}{l}\text { Bloomington (30032) } \\
\text { (Yu et al., 2010) }\end{array}$ \\
\hline$w^{-} ;$TRH-FIF/cyo; + & $\begin{array}{l}\text { Flippase expression mainly in } \\
\text { serotoninergic neurons }\end{array}$ & $\begin{array}{l}\text { Self-generated } \\
\text { (This thesis) }\end{array}$ \\
\hline$w^{-} ;+;$TRH-FIF/TM3 & $\begin{array}{l}\text { Flippase expression mainly in } \\
\text { serotoninergic neurons }\end{array}$ & $\begin{array}{l}\text { Self-generated } \\
\text { (This thesis) }\end{array}$ \\
\hline
\end{tabular}




\subsubsection{Gal4 lines from Janelia stock collection}

\begin{tabular}{|c|c|c|}
\hline Bloomington number & Description & Associated gene \\
\hline $38828^{*}$ & P\{GMR52D06-GAL4\}attP2 & $5-\mathrm{HT} 1 \mathrm{~B}$ \\
\hline 38843* & P\{GMR52G04-GAL4\}attP2 & $5-\mathrm{HT} 1 \mathrm{~A}$ \\
\hline $38870 *$ & P\{GMR53C03-GAL4\}attP2 & $5-\mathrm{HT} 1 \mathrm{~A}$ \\
\hline 38873 & P\{GMR53C10-GAL4\}attP2 & 5-HT1A \\
\hline 39087 & P\{GMR54G07-GAL4\}attP2 & Ace \\
\hline 39329 & P\{GMR65A05-GAL4\}attP2 & $\mathrm{Abl}$ \\
\hline 39349* & P\{GMR65D01-GAL4\}attP2 & $\mathrm{Abl}$ \\
\hline 39506* & P\{GMR70A02-GAL4\}attP2 & 5-HT7 \\
\hline $39514^{*}$ & P $\{$ GMR70B07-GAL4\}attP2 & $5-\mathrm{HT7}$ \\
\hline 39901* & P\{GMR75F06-GAL4\}attP2 & $a b$ \\
\hline $39902 *$ & P\{GMR75F07-GAL4\}attP2 & $a b$ \\
\hline 39938 & P\{GMR76G01-GAL4\}attP2 & amon \\
\hline 40419* & P\{GMR85B12-GAL4\}attP2 & $\mathrm{zf} 30 \mathrm{C}$ \\
\hline 46210 & P\{GMR36C02-GAL4\}attP2 & $A b d-B$ \\
\hline 46414 & P\{GMR58H04-GAL4\}attP2 & Ace \\
\hline 46534 & P\{GMR55E12-GAL4\}attP2 & Ace \\
\hline 46545 & P\{GMR64G04-GAL4\}attP2 & amn \\
\hline $46550 *$ & P\{GMR65A07-GAL4\}attP2 & AstC-R2 \\
\hline
\end{tabular}




\begin{tabular}{|c|c|c|}
\hline $46569 *$ & P\{GMR66A09-GAL4\}attP2 & amn \\
\hline $46576 *$ & P\{GMR67B05-GAL4\}attP2 & Abl \\
\hline 46627 & $\mathrm{P}\{\mathrm{GMR70A04-GAL4 \} attP2}$ & 5-HT7 \\
\hline $46628^{*}$ & $\mathrm{P}\{\mathrm{GMR70A05-GAL4 \} attP2}$ & $5-\mathrm{HT7}$ \\
\hline $46630 *$ & $\mathrm{P}\{\mathrm{GMR70A11-GAL4 \} attP2}$ & $5-\mathrm{HT7}$ \\
\hline $46938^{*}$ & $P\{G M R 75 D 10-G A L 4\} a t t P 2$ & $a b$ \\
\hline 46942 & $\mathrm{P}\{\mathrm{GMR75E} 10-\mathrm{GAL} 4\}$ attP2 & $a b$ \\
\hline $47447 *$ & P\{GMR10E03-GAL4\}attP2 & AstC-R2 \\
\hline 47711 & $\mathrm{P}\{\mathrm{GMR67C03-GAL4 \} attP2}$ & acj6 \\
\hline 47721 & $\mathrm{P}\{\mathrm{GMR70B05-GAL4 \} attP2}$ & $5-\mathrm{HT7}$ \\
\hline $47754^{*}$ & P $\{$ GMR76F10-GAL4\}attP2 & amon \\
\hline 48295 & P $\{$ GMR70B06-GAL4\}attP2 & $5-\mathrm{HT7}$ \\
\hline $48323 *$ & $\mathrm{P}\{\mathrm{GMR75D08-GAL4 \} attP2}$ & $a b$ \\
\hline 48448 & $\mathrm{P}\{\mathrm{GMR} 11 \mathrm{C07-GAL4 \} attP2}$ & AstC-R2 \\
\hline $48452^{*}$ & $P\{G M R 11 D 02-G A L 4\} a t t P 2$ & AstC-R2 \\
\hline $48970 *$ & $\mathrm{P}\{\mathrm{GMR} 22 \mathrm{~B} 11-\mathrm{GAL} 4\} \mathrm{attP2}$ & AstA-R1 \\
\hline 48978 & $\mathrm{P}\{\mathrm{GMR} 22 \mathrm{C} 12-\mathrm{GAL} 4\} \mathrm{attP} 2$ & AstA-R1 \\
\hline 49002 & $\mathrm{P}\{\mathrm{GMR} 22 \mathrm{H} 05-\mathrm{GAL} 4\}$ attP2 & AstA-R1 \\
\hline $49005^{*}$ & $\mathrm{P}\{\mathrm{GMR} 22 \mathrm{H} 10-\mathrm{GAL}$ \} $\}$ attP2 & AstA-R1 \\
\hline $49006^{*}$ & $\mathrm{P}\{\mathrm{GMR} 23 \mathrm{~A} 03-\mathrm{GAL} 4\}$ attP2 & AstA-R1 \\
\hline
\end{tabular}




\begin{tabular}{|c|c|c|}
\hline $49034^{*}$ & P\{GMR23E12-GAL4\}attP2 & AstA-R1 \\
\hline 49035 & $\mathrm{P}\{\mathrm{GMR} 23 \mathrm{~F} 05-\mathrm{GAL}$ \} $\}$ attP2 & Actbeta \\
\hline $49047^{*}$ & $\mathrm{P}\{\mathrm{GMR} 23 \mathrm{H} 03-\mathrm{GAL} 4\}$ attP2 & ari-1 \\
\hline 49122 & $\mathrm{P}\{\mathrm{GMR} 25 \mathrm{D} 01-\mathrm{GAL}$ \} $\}$ attP2 & tn \\
\hline 49129 & $\mathrm{P}\{\mathrm{GMR} 25 \mathrm{~F} 06-\mathrm{GAL}$ \} $\}$ attP2 & tn \\
\hline $49588^{*}$ & $\mathrm{P}\{\mathrm{GMR} 54 \mathrm{~A} 11-\mathrm{GAL} 4\}$ attP2 & $5-\mathrm{HT} 1 \mathrm{~A}$ \\
\hline 49616* & $P\{G M R 67 A 07-G A L 4\} a t t P 2$ & AstC-R2 \\
\hline $49824 *$ & P\{GMR10D09-GAL4\}attP2 & AstC-R2 \\
\hline 49899 & $\mathrm{P}\{\mathrm{GMR} 35 \mathrm{C07}-\mathrm{GAL} 4\}$ attP2 & zfh1 \\
\hline $49900 *$ & $\mathrm{P}\{\mathrm{GMR} 35 \mathrm{C08}-\mathrm{GAL} 4\}$ attP2 & Abd-B \\
\hline 49901* & P\{GMR35C09-GAL4\}attP2 & zfh1 \\
\hline $50441 *$ & P\{GMR53F06-GAL4\}attP2 & $5-\mathrm{HT} 1 \mathrm{~A}$ \\
\hline 50463 & $\mathrm{P}\{\mathrm{GMR} 54 \mathrm{~B} 02-\mathrm{GAL} 4\}$ attP2 & $5-\mathrm{HT} 1 \mathrm{~A}$ \\
\hline 49313* & P\{GMR24C05-GAL4\}attP2 & aos \\
\hline $47467^{*}$ & $\mathrm{P}\{\mathrm{GMR} 15 \mathrm{D} 07-\mathrm{GAL}$ \}attP2 & trh \\
\hline $49310^{*}$ & P\{GMR23H11-GAL4\}attP2 & ari-1 \\
\hline $46581^{*}$ & P\{GMR67E10-GAL4\}attP2 & AstA-R2 \\
\hline 48233* & $P\{G M R 65 D 03-G A L 4\} a t t P 2$ & Appl \\
\hline $46817^{*}$ & P\{GMR86B10-GAL4\}attP2 & $\mathrm{B}-\mathrm{H} 2$ \\
\hline 50403 & $\mathrm{P}\{\mathrm{GMR} 49 \mathrm{A09-GAL4 \} attP2}$ & beat-Ila \\
\hline
\end{tabular}




\begin{tabular}{lll}
\hline 47412 & P\{GMR80D03-GAL4\}attP2 & CG7587 \\
50342 & P\{GMR48A09-GAL4\}attP2 & beat-Vb \\
40652 & P\{GMR93D07-GAL4\}attP2 & beat-Vc \\
$48401^{*}$ & P\{GMR89A09-GAL4\}attP2 & beat-VI \\
$40547^{*}$ & P $\{$ GMR89C04-GAL4\}attP2 & beat-VI \\
\hline
\end{tabular}

* Expression of Gal4 in some serotoninergic neurons

\subsubsection{List of chemicals}

\begin{tabular}{lll}
\hline Chemical & Company & Catalogue no. \\
\hline Agar & Roth & 5210.2 \\
Agarose & Roth & 3820.4 \\
Ampicillin & Roth & K029.1 \\
Borate & Roth & 6943.1 \\
Bovine serum albumin & Roth & 8076.2 \\
Calcium chloride (CaCl2) & Roth & 5239.1 \\
Corn flour & Maismehl Obermühl Rosdorf & - \\
EDTA & Roth & CN06.2 \\
Ethanol & Merk & 100983 \\
Glucose & Applichem & A1377 \\
Hepes & Roth & 9105.2 \\
Hydrochloric acid $(\mathbf{H C l})$ & Th Geyer & 836 \\
\hline
\end{tabular}




\begin{tabular}{|c|c|c|}
\hline Isopropanol & AppliChem & A0900 \\
\hline Magnesium chloride (MgCl2) & Roth & 2189.2 \\
\hline Malt & Maismehl Obermühl Rosdorf & - \\
\hline Magnesium sulfate (MgSO4) & Roth & Po27.2 \\
\hline Nipagin & Sigma & H3647 \\
\hline Normal goat serum & Invitrogen & PCN5000 \\
\hline Para-chlorophenylalanine (PCPA) & Sigma & C6506 \\
\hline Paraformaldehyde & Roth & 0335.1 \\
\hline Potassium chloride ( $\mathrm{KCl})$ & Roth & 6781.3 \\
\hline Propionic acid & Roth & 6026.3 \\
\hline Red dye & Ruth & L3138 \\
\hline Sodium chloride( $\mathrm{NaCl})$ & AppliChem & A3597 \\
\hline Sodium hydroxide ( $\mathrm{NaOH})$ & Roth & K021.1 \\
\hline Sucrose & AppliChem & A4734 \\
\hline Tris & Roth & 48855.2 \\
\hline Triton-X-100 & Roth & 3051.2 \\
\hline Tryptone & Roth & 8952.2 \\
\hline Vectashield & Vector Laboratories & $\mathrm{H} 1000$ \\
\hline Treacle & Maismehl Obermühl Rosdorf & - \\
\hline
\end{tabular}




\subsubsection{Molecular biology reagents}

\begin{tabular}{|c|c|c|}
\hline Reagent & Company & Catalogue no. \\
\hline $6 x$ loading dye & Fermentas & R0611 \\
\hline Advantage $210 x$ buffer & Clontech & 639137 \\
\hline Advantage 2 polymerase & Clontech & 639201 \\
\hline DNA ladder (1Kb plus) & Fermentas & SM1331 \\
\hline dNTPs & Fermentas & R1122 \\
\hline Ethidium Bromide & Roth & 2218.3 \\
\hline Primers and oligos & Eurofins OMG & - \\
\hline QIAGEN Plasmid Midi Kit & Qiagen & 12145 \\
\hline QIAprep Spin Miniprep Kit & Qiagen & 27106 \\
\hline QIAquick Gel Extraction Kit & Qiagen & 28704 \\
\hline QIAquick PCR Purification Kit & Qiagen & 28104 \\
\hline Shrimp alkaline phosphatase (SAP) & Fermentas & EF0511 \\
\hline T4 DNA ligase & Fermentas & EL0014 \\
\hline T4 DNA polymerase & Fermentas & EP0061 \\
\hline XL1 blue compotent cells (E.coli) & Promega & 200249 \\
\hline Agel (BshTI) restriction enzyme & Fermentas & ER1461 \\
\hline Avrll (XmaJl) restriction enzyme & Fermentas & ER1561 \\
\hline Bglll restriction enzyme & Fermentas & ER0081 \\
\hline
\end{tabular}




\begin{tabular}{lll}
\hline BsiWI (PfI23II) restriction enzyme & Fermentas & ER0851 \\
BstEII restriction enzyme & Biolab & R0162S \\
Fsel restriction enzyme & Biolab & R0588S \\
Mlul restriction enzyme & Fermentas & ER0561 \\
Notl restriction enzyme & Fermentas & ER0591 \\
Pacl restriction enzyme & Biolab & R0547S \\
Sacll (Cfr42I) restriction enzyme & Fermentas & ER0205 \\
Spel (Bcul) restriction enzyme & Fermentas & ER1251 \\
Sphl (Pael) restriction enzyme & Fermentas & ER0601 \\
Xhol restriction enzyme & Fermentas & ER0691 \\
\hline
\end{tabular}




\subsubsection{List of antibodies}

\begin{tabular}{llll}
\hline Antibody & Used concentration & Company & Catalogue no. \\
\hline Rat anti RFP (monoclonal) & $1: 350$ & ChromoTek & 5 F8 \\
Rabbit anti 5-HT (polyclonal) & $1: 500$ & Sigma & S5545 \\
Mouse anti GFP (monoclonal) & $1: 750$ & Invitrogen & A11120 \\
Rabbit anti GFP(polyclonal) & $1: 750$ & Invitrogen & A6455 \\
Mouse NC82 (monoclonal) & $1: 10$ & Provided by Prof. Erich Buchner \\
Alexa Fluor 488 goat anti-rat & $1: 300$ & Invitrogen & A11006 \\
Cy3 goat anti-rat & $1: 500$ & Invitrogen & A10522 \\
Alexa Fluor 488 goat anti-mouse & $1: 200$ & Invitrogen & A11001 \\
Alexa Fluor 488 goat anti-rabbit & $1: 300$ & Invitrogen & A11034 \\
Alexa Fluor 633 goat anti-mouse & $1: 300$ & Invitrogen & A21050 \\
Alexa Fluor 633 goat anti-rabbit & $1: 300$ & Invitrogen & A21070 \\
Biotin goat anti-rat & $1: 200$ & Invitrogen & $62-9540$ \\
Alexa Fluor 488 streptavidin & $1: 300$ & Molecular probes & S11223 \\
\hline
\end{tabular}




\subsubsection{Devices}

\begin{tabular}{ll}
\hline Device & Company \\
\hline Bacteria incubator & Heraeus \\
Bacteria incubator with shaker & Sartorius \\
Centrifuge 5804R & Eppendorf \\
Centrifuge Pico 17 & Thermo Scientific \\
DAM system & Trikinetics \\
Electrophoresis set up & PeQlab \\
Fluorescent Stereo microscope & Zeiss \\
Fly incubator & Binder \\
Genoplex for gel documentation & VWR \\
Magnetic mixer & VWR \\
Microplate Spectrophotometer(Epoch) & Biotek \\
Microwave & Alaska \\
pH-meter & Schott \\
Pipettes & Eppendorf \\
Shaker & GFL \\
\hline Stereo microscope (stemi 2000) & Eppendorf \\
\hline
\end{tabular}




\begin{tabular}{ll}
\hline TissueLyser & Qiagen \\
UV transilluminator & Biostep \\
Vortex & IKA \\
Water bath & Memert \\
Weight & Sartorius \\
\hline
\end{tabular}

\subsubsection{Consumables}

\begin{tabular}{|c|c|c|}
\hline Consumable & Company & Catalogue no. \\
\hline Cover slips $18 \times 18 \mathrm{~mm}$ & Thermoscientific & L40931 \\
\hline Cover slips $24 \times 60 \mathrm{~mm}$ & Th Geyer & 7695031 \\
\hline Falcon tubes & Sarstedt & 62.547254 \\
\hline Fine forceps & F.S.T & $11252-20$ \\
\hline Food vial & Greiner bio-one & 205101, 217101, 960177 \\
\hline Microscope slides & Roth & 0656 \\
\hline Microtubes & Sarstedt & $72.691,72.690,72.699$ \\
\hline Min needles & F.S.T & $26002-10$ \\
\hline Pippet Tips & Sarstedt & $70.760002,70.762$ \\
\hline Serological pipette & Roth & N242.1 \\
\hline
\end{tabular}




\subsubsection{Fly Food}

\subsubsection{Standard fly food}

For $10 \mathrm{l}$ of the fly food the quantity of the ingredients is as follows:

$\begin{array}{lcl}\text { Agar } & 102.5 \mathrm{~g} & \\ \text { Soy flour } & 100 \mathrm{~g} & \\ \text { Yeast } & 180 \mathrm{~g} & \text { (Dissolved in 1l water) } \\ \text { Corn flour } & 800 \mathrm{~g} & \text { (Dissolved in 2l water) } \\ \text { Treacle } & 220 \mathrm{~g} & \text { (Dissolved in 1l water) } \\ \text { Malt } & 800 \mathrm{~g} & \text { (Dissolved in 1l water) } \\ \text { Propionic acid } & 63 \mathrm{ml} & \\ \text { Nipagin } & 15 \mathrm{~g} & \\ \text { Ethanol } & 50 \mathrm{ml} & \end{array}$

The agar was soaked in 51 water overnight. After adding soy flour, yeast, corn flour, treacle and malt to the agar, the mixture was boiled at $99.5^{\circ} \mathrm{C}$ for $2 \mathrm{~h}$. When the temperature of the mixture reached $55-60^{\circ} \mathrm{C}$ the propionic acid, nipagin and ethanol were added. The food was filled in to the plastic vials of three different sizes $(28 \mathrm{ml}, 68 \mathrm{ml}$ and $175 \mathrm{ml})$.

\subsubsection{Fly food containing para-chlorophenylalanine (PCPA)}

For preparing the PCPA-containing food, 60mg PCPA, 200 $\mu$ of 5\% sucrose solution, $200 \mu$ lap water and $15 \mu \mathrm{l}$ red dye were mixed with $1 \mathrm{ml}$ melted fly food in small vial. For a control $150 \mu \mathrm{l}$ $5 \%$ sucrose solution and $15 \mu \mathrm{l}$ of red dye were added to $1 \mathrm{ml}$ melted fly food. After the solidification of the food the vials were stored at $4^{\circ} \mathrm{C}$. 


\subsubsection{Fly food containing red dye for quantifying food uptake}

$1 \%$ agarose was added to the red dye and tap water respectively. The mixture was boiled in a microwave until the agarose was dissolved. After cooling down, 50\% 1M sucrose was added to the solution and mixed thoroughly. To each medium food vial, $5 \mathrm{ml}$ of the liquid was added. After solidification of the food, vials were stored at $4^{\circ} \mathrm{C}$ and pre-warmed $1 \mathrm{~h}$ before experiment. The vials were used within the $24 \mathrm{~h}$ after preparation.

\subsubsection{Buffers and solutions}

\subsubsection{For cloning}

\section{LB medium}

LB medium (lysogeny broth medium) is a rich medium for growing bacteria. For preparing 1 l of LB medium, $10 \mathrm{~g}$ tryptone, $5 \mathrm{~g}$ yeast extract and $10 \mathrm{~g} \mathrm{NaCl}$ were mixed with $950 \mathrm{ml}$ of $\mathrm{ddH}_{2} \mathrm{O}$. The $\mathrm{pH}$ of the medium was adjusted to 7.5 with $\mathrm{NaOH}$. The volume was filled up to one liter total volume and autoclaved.

\section{LB agar}

LB agar is the solidified LB medium for growing bacteria colonies. For 11 of LB medium, 15g of agar was dissolved in the $L B$ solution by autoclaving. After cooling to $50{ }^{\circ} \mathrm{C}, 1 \mathrm{ml}$ of ampicillin solution $(100 \mathrm{mg} / \mathrm{ml})$ was added to the medium and mixed thoroughly. The medium was poured into the petridishes and after solidification of the agar LB, the petridishes were stored at $4^{\circ} \mathrm{C}$.

\section{SOB medium}

SOB medium (super optimal broth) is a nutrient rich medium for growing bacteria. For preparing $1 \mathrm{l}$ of $\mathrm{SOB}$ medium, $20 \mathrm{~g}$ tryptone, $5 \mathrm{~g}$ yeast extract, $0.5 \mathrm{~g} \mathrm{NaCl}$ and $0.18 \mathrm{~g} \mathrm{KCl}$ were dissolved in $1 \mathrm{l}$ of $\mathrm{ddH}_{2} \mathrm{O}$. After autoclaving the solution, $10 \mathrm{ml}$ of $1 \mathrm{M} \mathrm{MgSO}_{4}$ and $10 \mathrm{ml}$ of $1 \mathrm{M}$ $\mathrm{MgCl}_{2}$ (filtered-sterilized) were added to the medium. It was stored in $4^{\circ} \mathrm{C}$ until usage. 


\section{SOC medium}

This medium is used for high efficiency bacterial transformation. For preparing $100 \mathrm{ml}$ of SOC medium, $1 \mathrm{ml}$ of $1 \mathrm{M}$ glucose solution (filtered-sterilized) was mixed with $99 \mathrm{ml}$ of SOB medium directly before usage.

\section{TBE (tris-borate-EDTA)}

TBE buffer was used for gel electrophoresis. For preparing 1 l of TBE buffer, $12.1 \mathrm{~g}$ Tris, $5.2 \mathrm{~g}$ borate and $0.4 \mathrm{~g}$ EDTA were dissolved in $1 \mathrm{l}$ of $\mathrm{dd}_{2} \mathrm{O}$. The $\mathrm{pH}$ was adjusted to 8.0 using $\mathrm{NaOH}$ and $\mathrm{HCl}$.

\subsubsection{For immunohistochemistry}

\section{Ringer's solution}

For creating an isotonic solution relative to the body fluids and extending the survival time of the dissected tissues, Ringer's solution was used for brain dissection. For preparing 11 of this buffer, $1.19 \mathrm{~g}$ Hepes, $7.6 \mathrm{~g} \mathrm{NaCl}, 0.4 \mathrm{~g} \mathrm{KCl}, 0.4 \mathrm{~g} \mathrm{MgCl}_{2}, 0.3 \mathrm{~g} \mathrm{CaCl}_{2}$ and $12.3 \mathrm{~g}$ sucrose were dissolved in $1 \mathrm{dddH_{2 }} \mathrm{O}$ and the $\mathrm{pH}$ was adjusted to 7.3 with $\mathrm{NaOH}$. Using an osmometer, the osmalarity of the buffer was adjusted to 290-310 mOsmol by adding $\mathrm{NaCl}$. The solution was aliquoted in $50 \mathrm{ml}$ Falcon tube and stored at $-20^{\circ} \mathrm{C}$.

\section{PBS}

For preparing $1 \mathrm{l}$ of $1 \times \mathrm{PBS}$ buffer, $8 \mathrm{~g} \mathrm{NaCl}, 0.2 \mathrm{~g} \mathrm{KCL}, 1.44 \mathrm{~g} \mathrm{Na}{ }_{2} \mathrm{HPO}_{4}$ and $0.24 \mathrm{~g} \mathrm{KH}_{2} \mathrm{PO}_{4}$ were

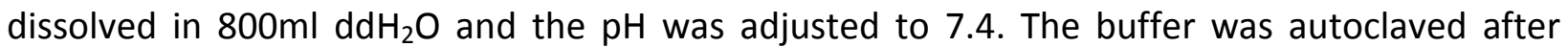
adjusting the volume to 1 l by adding $\mathrm{ddH}_{2} \mathrm{O}$.

\section{Fixative (4\% paraformaldehyde)}

$25 \mathrm{ml}$ of $1 \mathrm{xPBS}$ was heated to $60^{\circ} \mathrm{C}$. $1 \mathrm{~g}$ paraformaldehyde (PFA) was dissolved in the heated PBS. For complete dissolving of PFA in the PBS, $2.5 \mu \mathrm{l}$ of $10 \mathrm{M} \mathrm{NaOH}$ was added to the mixture. Afterward, the pH was adjusted to 7.4 and the bottle of the solution was cooled down on ice. The $4 \%$ PFA was aliquoted in $1.5 \mathrm{ml}$ microtubes and stored at $-20^{\circ} \mathrm{C}$. 


\section{PBT (PBS and Triton-X-100)}

For washing steps in immunostaining 0.6\% PBT was used. For preparing this buffer 0.6\% Triton$X-100$ was mixed with PBS buffer.

\section{PAT (PBT and BSA)}

$2 \%$ bovine serum albumin (BSA) was mixed thoroughly with PBT. This solution can be kept 2-4 days at $4^{\circ} \mathrm{C}$.

\section{Block solution}

The block solution was prepared by adding 5\% normal goat serum (NGS) to PAT solution freshly before usage.

\subsection{Methods}

\subsubsection{Fly maintenance}

\section{Standard maintenance}

Flies were raised on standard fly food (section 2.1.8.1) in fly incubators at $18^{\circ} \mathrm{C}$ or $25^{\circ} \mathrm{C}$ with $60 \%$ humidity and at 12/12h light-dark cycle conditions. All flies expressing dTRPA 1 and the respective experimental controls were raised at $18^{\circ} \mathrm{C}$. Depending on the number of the flies, three different sizes for plastic vials with fly food were used $(28 \mathrm{ml}, 68 \mathrm{ml}$ and $175 \mathrm{ml})$. The parental lines for crosses were transferred to new vials every 3-5 days.

\section{Fly starvation}

Medium vials $(68 \mathrm{ml})$ containing tissue paper moistened with tap water in the bottom of the vials were used for starving the flies. 20-25 flies were transferred to the vial and were starved for $24 \mathrm{~h}$ at $25^{\circ} \mathrm{C}$ or $48 \mathrm{~h}$ at $18^{\circ} \mathrm{C}$, respectively. 


\section{Heat-shocking}

For inducing expression of the heat-shock flippase (Basler and Struhl, 1994), 1-2 days old flies were transferred to a $32^{\circ} \mathrm{C}$ warm water-bath for $1 \mathrm{~h}$. After heat-shocking the flies, they were kept at $18^{\circ} \mathrm{C}$. After four days, the flies were used for anatomical or behavioral tests.

\subsubsection{Generating transgenic flies}

\subsubsection{Cloning procedure}

\section{A. Amplification of the insert and adding restriction cutting sites to $5^{\prime}$ and $3^{\prime}$ ends}

Polymerase chain reaction (PCR) was performed for amplifying DNA inserts using the Clontech "Advantage 2" polymerase enzyme. For PCR reaction, template DNA (100-200 ng), 5 $\mu$ l of 10x "Advantage 2" PCR buffer, $1 \mu$ l of dNTP mix (10mM each), $1 \mu$ l of each forward and reverse primers $(25 \mathrm{pmol} / \mu \mathrm{l}), 1 \mu \mathrm{l}$ of "Advantage 2 " polymerase and nuclease free water to a final volume of $50 \mu$ l were mixed and kept on ice.

A two-step protocol was used for amplification of the inserts. Depending on the size of the insert, the annealing/extension times were different in the two-step PCR.

\begin{tabular}{|l|l|l|l|}
\hline Cycle step & Temp. & Time & Number of cycles \\
\hline Initial denaturation & $95^{\circ} \mathrm{C}$ & $5 \mathrm{~min}$ & $1 \mathrm{cycle}$ \\
\hline Denaturation & $95^{\circ} \mathrm{C}$ & $30 \mathrm{sec}$ & \\
Annealing/ extension & $68^{\circ} \mathrm{C}$ & $1 \mathrm{~min}$ to $6 \mathrm{~min}$ & \\
\hline Final extension & $68^{\circ} \mathrm{C}$ & $1 \mathrm{~min}$ to $6 \mathrm{~min}$ & 1 cycle \\
\hline
\end{tabular}




\begin{tabular}{|l|l|}
\hline Target size & $\begin{array}{l}\text { Annealing/ extension } \\
\text { and final extension }\end{array}$ \\
\hline $\mathbf{> 1} \mathbf{~ K b}$ & $1 \mathrm{~min}$ \\
\hline $\mathbf{1 - 5} \mathbf{~ k b}$ & $3 \mathrm{~min}$ \\
\hline $\mathbf{5 - 9} \mathbf{~ K b}$ & $6 \mathrm{~min}$ \\
\hline
\end{tabular}

The sequences of the primers are indicated in cloning section 2.2.2.2 for each fragment.

\section{B. Gel electrophoresis}

After the PCR reaction, the products were visualized using gel electrophoresis. 3-5 $\mu$ l of each PCR product was mixed with $6 x$ loading dye and electrophoretically separated by size in the $1 \%$ agarose gel. Together with the PCR product, $5 \mu \mathrm{l}$ of $1 \mathrm{~kb}$ plus ladder was loaded to the gel to determine the size of the sample.

\section{PCR product purification}

After confirming the PCR product using gel electrophoresis, sometimes a purification of the insert was required. When a single-band PCR product was obtained, the PCR purification kit from Qiagen was used to eliminate primer dimers (protocol in section 2.2.2.3). But in the case of multiple bands, the DNA fragment with the right size was cut out from the agarose gel and purified using Qiagen gel extraction kit (protocol in section 2.2.2.3).

\section{Restriction digest of the insert and vector}

For producing "sticky ends" in the insert and vector, suitable restriction enzymes and buffers were used. The mixture of DNA, restriction enzymes, buffer and nuclease free water to a final volume of $50 \mu \mathrm{l}$ were incubated at $37^{\circ} \mathrm{C} 2$ to $3 \mathrm{~h}$ (depended on the DNA concentration).

\section{E. Alkaline phosphatase treatment}

For preventing a linear vector from self-ligation, shrimp alkaline phosphatase (SAP) was used to remove the 5 'phosphate from the DNA ends. This reaction was done directly after restriction 
digestion by adding $1 \mu$ of SAP enzyme to the restriction digest mixture and incubated for 30 $\min$ at $37^{\circ} \mathrm{C}$. The reaction was stopped by heating for $15 \mathrm{~min}$ to $65^{\circ} \mathrm{C}$.

\section{F. Ligation}

For ligation, insert and vector (molar ratio 3:1) were mixed with 3 $\mu$ l of 10x T4 DNA ligase buffer, $2 \mu \mathrm{l}$ of T4 DNA ligase enzyme and nuclease free water to a final volume of $30 \mu \mathrm{l}$. The reaction was incubated for $1 \mathrm{~h}$ at room temperature or $12 \mathrm{~h}$ at $18^{\circ} \mathrm{C}$. For adaptor ligation, $3 \mu \mathrm{l}$ of $50 \%$ PEG 4000 Solution was added to the reaction. The reaction was stopped by heating to $65^{\circ} \mathrm{C}$ for 10 $\min$.

\section{G. Bacterial transformation}

The DNA was transformed to XL1 Blue chemically competent cells. One aliquot of XL1 blue bacteria $(200 \mu \mathrm{l})$ was thawed on ice and $10 \mu \mathrm{l}$ of the ligation mixture was added to the cells followed by incubation on ice for $30 \mathrm{~min}$. The cells were treated with a heat-shock at $42^{\circ} \mathrm{C}$ water-bath for 45 seconds and incubated on ice for $2 \mathrm{~min}$ again. $900 \mu \mathrm{l}$ of pre-warmed SOC medium was added to the cells and the vial was incubated for $1 \mathrm{~h}$ at $37^{\circ} \mathrm{C}$ with shaking at 225 $250 \mathrm{rpm}$. In the next step the $50 \mu \mathrm{l}$ of medium were plated on LB agar plate containing $100 \mu \mathrm{g} / \mathrm{ml}$ ampicillin. The LB agar plate was incubated at $37^{\circ} \mathrm{C}$ overnight.

\section{H. Isolating the plasmid from a bacteria colony}

From the agar plate, 5 single colonies were picked and cultured in $5 \mathrm{ml}$ of LB medium containing $100 \mu \mathrm{g} / \mathrm{ml}$ ampicillin. After overnight incubation of $\mathrm{LB}$ medium in $37^{\circ} \mathrm{C}$ with shaking at $250 \mathrm{rpm}$, $2 \mathrm{ml}$ of the culture was used for plasmid purification using the Qiagen miniprep kit (protocol in section 2.2.2.3). After the isolation of DNA and determining the concentration using a nanodrop device, the ligation of the vector with the insert was checked by analytical restriction digest and gel electrophoresis. $200 \mathrm{ml}$ of the fresh LB medium containing $100 \mu \mathrm{g} / \mathrm{ml}$ ampicillin was inoculated with $200 \mu \mathrm{l}$ of the bacterial culture from the correct colony. The LB culture was incubated at $37^{\circ} \mathrm{C}$ overnight with shaking at $250 \mathrm{rpm}$. The plasmid at a concentration of 1$2 \mu \mathrm{g} / \mu \mathrm{l}$ was isolated from the LB culture using the Qiagen midiprep kit (protocol in section 2.2.2.3). 


\section{DNA sequencing}

The sequence of the inserts in the generated vectors was confirmed by double-stranded DNA sequencing performed by Eurofins MWG Operon.

\section{J. Plasmid microinjection}

Plasmids contains the appropriate inserts were sent to BestGene Inc. to perform germline transformation of Drosophila and to screen for stable and balanced transgenic lines. The inserts in the pUAST vectors were transformed to the fly genome based on P-element transgenesis (Rubin and Spradling, 1982). However, the transformation of inserts in pBDP vectors was based on the PhiC31 integrase-mediated transgenesis systems (Groth et al., 2004). In this system, the site-specific integration between bacterial attachment site (attB) in the pBDP vector and phage attachment site (attP) in the embryo was mediated by a bacteriophage PhiC31 integrase.

\subsubsection{Cloning strategy for different vectors}

\section{I. pUAST-dTRPA1-mCherry vector:}

The mCherry (red fluorescent protein) was amplified from pcDNA3-NES/ cAMP sponge-mCherry vector (provided by Dr. Hofer; Lefkimmiatis et al., 2009). Using the following primers, Notl and Mlul restriction cutting sites were added to the $5^{\prime}$ end and Xhol restriction cutting site to the $3^{\prime}$ end of the mCherry:

mCherry forward 1: 5'-GGACGCGTATGGTGAGCAAGGGCGAG-3'

mCherry forward 2: 5'-GCGGCCGCGGACGCGTATGGTGAGCAAGG-3'

mCherry forward 3: 5'-ATAAGAAT GCGGCCGCGGACGCGTATGGT-3'

mCherry reverse: 5'-GGCTCGAGTTACTTGTACAGCTCGTCCAT-3'

The mCherry was cloned into the pUAST vector using Notl and Xhol restriction cutting sites. In this construct the mCherry tag is located in the C-terminal of the dTRPA1 protein. The dTRPA1 was amplified from pOX-dTRPA1 vector (provided by Dr. Garrity; Rosenzweig et al., 2005) using the following linker primers: 
dTRPA1 forward: 5'-GGAGATCTATGACTTCGGGCGACAAG-3'

dTRPA1 reverse: 5'-GGACGCGTCATGCTCTTATTGAAGCTCAG-3'

With the linker PCR, BgIII and Mlul restriction cutting sites were added to dTRPA1. The dTRPA1 cDNA in this construct did not have a stop codon. Using the BgIII and Mlul restriction cutting sites, this fragment was inserted to the pUAST-mCherry vector.

\section{II. pUAST-mCherry-dTRPA1 vector:}

In this vector the mCherry is not following by a stop codon and is located upstream of the dTRPA1. A Bglll restriction cutting site was added to the $5^{\prime}$ end and a Notl and a Spel restriction cutting site to the $3^{\prime}$ end of the mCherry using following linker primers:

mCherry forward: 5'-GAAGATCTATGGTGAGCAAGGGCGAGGAG-3'

mCherry reverse1: 5'-GGACTAGTCTTGTACAGCTCGTCCAT-3'

mCherry reverse2: 5'-GCGGCCGCGGACTAGTCTTGTACAGCTCG-3'

mCherry reverse3: 5'-ATAAGAATGCGGCCGCGGACTAGTCTTGT-3'

The mCherry gene was cloned into the PUAST vector using Bglll and Notl restriction cutting sites. Spel and Xhol restriction cutting sites were introduced into the dTRPA1 using the following linker primers:

dTRPA1 forward: 5'-GGACTAGTATGACTTCGGGCGACAAG-3'

dTRPA1 reverse: 5'-GGCTCGAGCTACATGCTCTTATTGAAGCT-3'

The dTRPA1 was cloned into the pUAST-mCherry vector in the BgIII and Notl restriction cutting sites.

\section{III. pUAST-FRT-CD2-stop-FRT-mCherry-dTRPA1vector:}

The pUAST vector with FRT-CD2-y+-stop-FRT cassette (FRT cassette) was provided kindly by Prof. Gary Struhl (Basler and Struhl, 1994). Part of the $y+$ sequence was omitted from the vector using BstEll and Pacl restriction cutting sites. The protocol for ligation of the linear vector with two different sticky ends after each excision is described in section 2.2.2.3. 
The multiple cloning site was inserted downstream of the FRT cassette an using adaptor with ACC65I, BsiWI, Rsrll and Sacll restriction cutting sites (the protocol is described in section 2.2.2.3). The sequence of single strand oligo nucleotides were as follows:

Adaptor strand 1: 5'-pGTACCACGTACGTCGGACCGTCCCGCGGGGACCGC-3'

Adaptor strand 2: 5'-pGGTCCCCGCGGGACGGTCCGACGTACGTG-3'

The mCherry-dTRPA1 construct was amplified from the pUAST-mCherry-dTRPA1 vector (section 2.2.2.2 II) using following linker primers:

mCherry-dTRPA1 forward: 5'-AATCGTACGATGGTGAGCAAGGGCGAGG-3'

mCherry-dTRPA1 reverse: 5'-GGAGGCCGCGGCTACATGCTCTTATTGAA-3'

The mCherry-dTRPA1 was cloned downstream of the FRT cassette using BsiWI and Sacll restriction cutting sites.

\section{PBDP-TRH -FIF vector:}

For generation of the pBDP-TRH-FIF vector, the TDC2 promoter in PBDP-TDC2-FIF vector was replaced by TRH promoter (provided kindly by Dr. Kravitz; Alekseyenko et al., 2010). The cloning strategy for generation of pBDP-TDC2-FIF vector is as follows:

The pBDP-TDC2-Gal4 vector was provided kindly by Dr. Rubin. The TDC2 promoter consists of two fragments (upstream and downstream of the Gal4). The Gal4 fragment flanked by Fsel and Spel restriction cutting sites was cut out and replaced by an adaptor. The adaptor which were consisted of Fsel, Mlul, Agel,Aatll and Spe1 restriction cutting sites were generated with the following single strand oligo nucleotides (the protocol is described in section 2.2.2.3):

Adaptor strand 1: 5'-pCCTACGCGTCTACCGGTCAGACGTCAGA-3'

Adaptor strand 2: 5'-pCTAGTCTGACGTCTGACCGGTAGACGCGTAGGCCGG-3'

FIF fragment consists of two copies of flippase which is separated with IRES sequence and SV40 sequence at the $3^{\prime}$ end (Flippase-IRES-Flippase-SV40). It was amplified from FIF vector (provided kindly by Dr. Zhang; Bohm et al., 2010) using following linker primers:

FIF forward: 5'-GGACGCGTTGCACGTTTGCTTGTTGAGAG-3' 
FIF reverse: 5'-CCACCGGTGATGAGTTTGGACAAACCACA-3'

Using Mlul and Agel restriction cutting sites FIF fragments was cloned into the pBDP-TDC2 vector.

For producing pBDP-TRH-FIF vector, TDC2 promoter fragments were cut out using suitable restriction cutting sites. Fragment 1 was flanked by Avrll and Fsel and fragment 2 was flanked by Agel and BgllI. The protocol for ligation of the linear vector with two different sticky ends after each excision is described in section 2.2.2.3. An adaptor consisting of AVrll, BsiWI, Sphl and Fsel was inserted upstream of the FIF fragment (the protocol is described in section 2.2.2.3). The sequence of single strand primers adaptors were as follows:

Adaptor strand 1: 5' -pCTAGGTCGTACGTATGCATGCTATGGCCGG-3'

Adaptor strand 2: 5'-pCCATAGCATGCATACGTACGAC-3'

The TRH promoter was amplified from TRH-Gal4 vector (provided kindly by Dr. Kravitz; Alekseyenko et al., 2010) using following linker primers:

TRH forward: 5'-TACGTACGATAAAAGTAAATATCTGGTAC-3'

TRH reverse: 5'-ATGCATGCCTTGGTAGCTACTCGTTTTCG-3'

Using BsiWI and Sphl restriction cutting sites TRH promoter was cloned to pBDP-FIF vector.

\subsubsection{Molecular protocols}

\section{Qiagen PCR purification}

PCR product and PBI buffer (1:5) were mixed and transferred to the QIAquick spin column. For binding the DNA to the column, it was centrifuged at $13000 \mathrm{rpm}$ for $1 \mathrm{~min}$. The flow-through was discarded and $750 \mu \mathrm{l}$ of buffer PE was added to the column. The column was centrifuged at $13000 \mathrm{rpm}$ for $1 \mathrm{~min}$ and the flow-through was discarded again. For removing all the ethanol from the DNA the column was centrifuged for additional $1 \mathrm{~min}$. The column was placed in a clean $1.5 \mathrm{ml}$ microtube. $20 \mu \mathrm{l}$ to $30 \mu \mathrm{l}$ of the EB buffer (based on the desired concentration) was added to the column and after 5 min incubation the column was centrifuged at 13000 rpm for 1 min. 


\section{Qiagen gel extraction}

The DNA band was cut out from 1\% agarose gel using an UV transilluminator for band visualization and placed in a clean 1.5 microtube. The weight of the agarose gel was measured and the QG buffer (3 volumes) was added to 1 volume of the agarose gel (e.g. 300 $\mu$ l of the QG buffer for $100 \mathrm{mg}$ of the agarose gel). The microtube was incubated in the thermomixer at $50^{\circ} \mathrm{C}$ for $10 \mathrm{~min}$ (shaking at $250 \mathrm{rpm}$ ) until the agarose gel was dissolved thoroughly. 1 gel volume of isopropanol (e.g. $100 \mu \mathrm{l}$ of the isopropanol for $100 \mathrm{mg}$ of the agarose gel) was added to the sample and mixed. The mixture was transferred to the QIAquick column. For binding the DNA to the column, it was centrifuged at $13000 \mathrm{rpm}$ for $1 \mathrm{~min}$. The flow-through was discarded and $500 \mu \mathrm{l}$ of QG buffer was added for further washing of the DNA from the agarose gel. After $1 \mathrm{~min}$ centrifuging at $13000 \mathrm{rpm}$ the flow-through was discarded and the column was washed with $750 \mu l$ of the PE buffer. After 5 min incubation of PE buffer in the column it was centrifuged at $13000 \mathrm{rpm}$ for $1 \mathrm{~min}$. The flow-through was discarded and for complete removal of PE buffer the column was centrifuged again for $1 \mathrm{~min}$. The column is placed into a clean $1.5 \mathrm{ml}$ microtube and $20-30 \mu \mathrm{l}$ of the EB buffer was added to the column. After 5 min incubation, the column was centrifuged at $13000 \mathrm{rpm}$ for $1 \mathrm{~min}$.

\section{Qiagene miniprep}

$2 \mathrm{ml}$ of the bacteria culture was centrifuged at $5000 \mathrm{rpm}$ in the microtube. The bacterial pallet was resuspended in $250 \mu \mathrm{l}$ of the P1 buffer by vortexing. $250 \mu \mathrm{l}$ of the P2 buffer was added to the sample and mixed thoroughly by inverting the tube 4-6 times. In the next step, 350 $\mu$ lof the N3 buffer was added and mixed immediately by inverting the tube 4-6 times. The solution should become cloudy. The microtube was centrifuged for $10 \mathrm{~min}$ at $13000 \mathrm{rpm}$. The supernatant was transferred to the QIAprep spin column and centrifuged for $1 \mathrm{~min}$ at 13000 rpm. The flow-through was discarded and the column was washed by adding $500 \mu$ of the PB buffer and centrifuging for $1 \mathrm{~min}$ at $13000 \mathrm{rpm}$. The flow-through was discarded and 750 $\mu$ of the PE buffer was added to the column. The column was centrifuged for $1 \mathrm{~min}$ and after discarding the flow-through, the column was centrifuged again for complete removal of PE buffer. The column was placed in a clean $1.5 \mathrm{ml}$ microtube and $50 \mu \mathrm{l}$ of the EB buffer was added. After 5 min incubation time the column was centrifuged for $1 \mathrm{~min}$ at $13000 \mathrm{rpm}$. 


\section{Qiagene midiprep}

$100 \mathrm{ml}$ of the bacteria culture was centrifuged at $5000 \mathrm{rpm}$ for $15 \mathrm{~min}$ at $4^{\circ} \mathrm{C}$ and the bacteria pallet was resuspended in $4 \mathrm{ml}$ of the $\mathrm{P} 1$ buffer by vortexing. The mixture was transferred to the $50 \mathrm{ml}$ falcon tube and $4 \mathrm{ml}$ of the P2 buffer is added. The buffer was mixed by inverting the falcon tube 4-6 times. The tube was incubated at room temperature for $5 \mathrm{~min}$. After incubation time, $4 \mathrm{ml}$ of the chilled P3 buffer was added and mixed by inverting the tube 4-6 times. Adding P3 buffer produced the fluffy white material. The falcon tube was incubated on ice for $15 \mathrm{~min}$. After incubation time the falcon was centrifuged at $7000 \mathrm{rpm}$ for $45 \mathrm{~min}$ at $4^{\circ} \mathrm{C}$. All the fluffy material should be deposit in the bottom of the falcon and the supernatant should be clear. The supernatant was transferred to the clean falcon tube. If the supernatant was not clear, it was centrifuged again for $15 \mathrm{~min}$ at $4^{\circ} \mathrm{C}$. For equilibrating the QIAGEN-tip 100 column, $4 \mathrm{ml}$ of the QBT buffer was added. The column should be empty by gravity flow. The supernatant was added to the equilibrated QIAGEN-tip 100 column and allowed to pass the column by gravity flow. After binding the DNA to the column, it was washed two times with $10 \mathrm{ml}$ of the QC buffer. The washing buffer moves through the QIAGEN-tip 100 column by gravity flow. The column was placed into a clean $15 \mathrm{ml}$ falcon tube and $5 \mathrm{ml}$ of the elution buffer (QF) was added. The elution buffer passing through the column by gravity flow was collected. The DNA in the elution buffer was precipitated by adding $4 \mathrm{ml}$ of the isopropanol. After mixing, the falcon tube was centrifuged immediately at $7000 \mathrm{rpm}$ for $30 \mathrm{~min}$ at $4^{\circ} \mathrm{C}$. The supernatant was discarded carefully and $2 \mathrm{ml}$ of the $70 \%$ ethanol was added to the DNA pallet. After 10 min centrifuging at $4^{\circ} \mathrm{C}(7000 \mathrm{rpm})$, the supernatant was discarded carefully. The DNA pallet in the falcon tube was air-dried in $37^{\circ} \mathrm{C}$ incubator (until the ethanol was removed). $100-200 \mu l$ of the TE buffer was added to the pallet for dissolving the DNA.

\section{Constructing an adaptor-duplex}

The two strands of oligo nucleotides functioning as adaptor should be complementary and designed in a way to make sticky ends at $5^{\prime}$ and $3^{\prime}$ restriction cutting sites after hybridization. The $5^{\prime}$ phosphate groups on both strands were required. The two adaptor strands with equimolar concentration were mixed in a buffer with low amounts of a monovalent cation. The online software from Promega (ssDNA: pmol $/ \mu \mathrm{l}$ to $\mu \mathrm{g} / \mathrm{ml}$ ) was used for calculation of the single strand concentration and T4 ligase buffer were used as annealing buffer. The adaptor strands mixture $(10 \mu \mathrm{g}$ of each strand, $3 \mu \mathrm{l}$ of the $10 x \mathrm{~T} 4$ ligase buffer and nuclease free water to make 
the final volume of $30 \mu \mathrm{l}$ ) was heated to $95^{\circ} \mathrm{C}$ for $2 \mathrm{~min}$ and slowly cooled down to $50^{\circ} \mathrm{C}$. Two oligos were hybridized in $5 \mathrm{~min}$ at $50^{\circ} \mathrm{C}$.

\section{Ligation of a linear vector with two different sticky ends}

For ligating a linear vector with two different sticky ends, both blunt 5'and 3'overhangs were required. This technique was also applicable for deleting one restriction cutting sites from the vector. After cutting the vector with suitable restriction enzymes, T4 DNA polymerase can be used for blunting the $5^{\prime}$ and 3 'ends. For the reaction, $4 \mu \mathrm{l}$ of $5 \mathrm{x}$ reaction buffer, $1 \mu \mathrm{g}$ of the linear vector, $1 \mu \mathrm{l}$ of dNTP mix ( $2 \mathrm{mM}$ each), $0.2 \mu \mathrm{l}$ of T4 DNA polymerase and nuclease free water to a final volume of $20 \mu \mathrm{l}$ were mixed thoroughly and incubated for $5 \mathrm{~min}$ at room temperature. The reaction was stopped by heating to $75^{\circ} \mathrm{C}$ for $10 \mathrm{~min}$.

\subsubsection{Evaluation of transgenic lines}

For each generated fly strain, several transgenic fly lines were received from the Bestgene Inc. company These versions were different in the insertion position of the transgene in the fly genome (in the lines which were generated based on P-element transgenesis). Because of the position-effect, for each transgene all lines with P-element insertion in different chromosomes were tested for their gene expression efficiency.

UAS:dTRPA1-mCherry and UAS:mCherry-dTRPA1 lines were evaluated by expressing these transgenes under the control of the actin promoter using actin-Gal4. Flies were tested in paralysis paradigm (section 2.2.5.1). For testing for a possible leakiness of the transgene expression, the brains of the UAS lines (without crossing to the Gal4 diver line) were analyzed using confocal microscopy.

The expression efficiency of different lines for UAS:FRT-CD2-stop-FRT-mCherry-dTRPA1 transgene was tested by crossing them with the hs-FLP; actin-Gal4/cyo line. The larvae from this cross were exposed to the $12 \mathrm{~h}$ heat-shock at $32^{\circ} \mathrm{C}$ and in the 5 days old offspring the fluorescence caused by mCherry was checked using fluorescence microscopy. The line with highest expression efficiency and clear viability was chosen for the experiments. 


\subsubsection{Immunohistochemistry}

\subsubsection{Standard immunohistochemistry}

Whole mount brains and, in some cases, the thoracic ganglions were dissected in Ringer's solution using fine forceps. The tissues were fixed in $4 \%$ paraformaldehyde (PFA) for $2 \mathrm{~h}$ at $4^{\circ} \mathrm{C}$ while shaking. Incubation was performed while samples were placed on the rotor for shaking. After fixation the tissues were washed 3 times 10 min each with $0.6 \%$ PBT at room temperature. Afterwards, the tissues were blocked with blocking solution (2\% PAT and 5\% NGS) for 2 hours at room temperature. The primary antibody or antibodies diluted in the blocking solution was added to the tissues. The samples were incubated in the primary antibody overnight at $4^{\circ} \mathrm{C}$. On the second day samples were washed 3 times for at least 30 min each at room temperature. The secondary antibody or antibodies diluted in 2\% PAT solution was added to the tissues. The samples were incubated $4 \mathrm{~h}$ either at room temperature or overnight at $4^{\circ} \mathrm{C}$. The tissues were then washed at least 6 times for 30 min each in $0.6 \%$ PBT. The brains and thoracic ganglia were mounted in vectashield, covered with coverslips and sealed with nail polish.

For antibody staining against mCherry, rat anti-RFP as a primary antibody and Alexa Fluor 488coupled goat anti-rat or Cy3-coupled goat anti-rat as secondary antibodies were used. For antibody staining against GFP, mouse anti-GFP or rabbit anti-GFP as primary antibody and Alexa Fluor 488-coupled goat anti-mouse or Alexa Fluor 488-coupled goat anti-rabbit as secondary antibody were used. For antibody staining against serotonin, rabbit anti-5-HT as primary antibody and Alexa Fluor 633-coupled goat anti-rabbit as secondary antibody were used. For antibody staining against bruchpilot, mouse anti-NC82 as primary antibody and Alexa Fluor 633coupled goat anti-mouse as secondary antibody were used.

\subsubsection{Enhanced immunohistochemistry}

After stochastically expressing mCherry-dTRPA1 in serotoninergic neurons, an enhanced immunohistochemistry (biotin-streptavidin) system was used for enhanced visualization of the neurons. For this staining technique, the brains were fixed and blocked as described in section 2.2.3.1. A mixture containing the rat anti-RFP and rabbit anti-5-HT primary antibodies diluted in the blocking solution was added to the tissues. The samples were incubated in the primary antibodies overnight at $4^{\circ} \mathrm{C}$. On the second day the samples were washed at least 3 times for 30 
min each at room temperature. After washing, biotin anti-rat antibody diluted in blocking solution was added to the samples and incubated $3 \mathrm{~h}$ at room temperature. After incubation time, the samples were washed 3 times for $30 \mathrm{~min}$ each with PAT solution at room temperature. In the next step, Alexa Fluor 488 streptavidin and Alexa Fluor 633-coupled goat anti-rabbit antibodies diluted in $2 \%$ PAT solution were added to the brains. After overnight incubation at $4^{\circ} \mathrm{C}$, the tissues were washed at least 6 times for $30 \mathrm{~min}$ in $0.6 \%$ PBT. The brains were mounted in the vectashield, covered with coverslips and sealed with nail polish.

\subsubsection{Microscopy}

\subsubsection{Wide-field (WF) microscopy}

For comparing the fluorescence intensity of the different antennae, they were scanned using wide-field fluorescence microscope (Zeiss Axioskop 2 FS) equipped with a xenon lamp (Lambda DG-4, Shutter Instrument), a 14 bit CCD camera (Coolsnap HQ, Photometrics), a 20x 0.7 NA dry objective and a RFP filter set. Image acquisition was controlled using the Metafluor software (Visitron Systems).

The images of the entire flies were obtained using fluorescence stereo microscope (Discovery. V8, Zeiss) equipped with a CCD camera (AxioCam, Zeiss).

\subsubsection{Confocal laser scan microscopy}

The immunostained samples were scanned using a Leica TC SP2 confocal microscope equipped with Leica apochromat 20x 0.7 NA water-immersion objective. The Alexa 488 was excited at $488 \mathrm{~nm}$ (argon laser), Cy3 at $543 \mathrm{~nm}$ (HeNe 543nm laser), and Alexa 633 at $633 \mathrm{~nm}$ (HeNe $633 \mathrm{~nm}$ laser) wavelength. Samples were scanned at $1 \mu \mathrm{m}$ steps in the $z$ axis at a frame averaging 4 and with a resolution of $0.57 \mu \mathrm{m} /$ pixel. All images were acquired as 12-bit grayscale.

\subsubsection{Two-photon microscopy}

For comparing different samples for the intensity of $5-\mathrm{HT}$ antibody staining, brains were scanned using a Zeiss LSM7 MP two-photon microscope equipped with Zeiss w-plan apochromat 20x water immersion objective $(N A=1.0)$ and using a dichroic mirror in combination with 500-550 nm and 590-710 nm emission filters. The images were acquired 
with excitation wavelengths of $920 \mathrm{~nm}$, frame average of $4,1 \mu \mathrm{m}$ steps size in the $\mathrm{z}$ axis and a resolution of $0.6 \mu \mathrm{m} /$ pixel.

\subsubsection{Image processing}

The image stacks obtained from confocal or two-photon microscopy were processed using the Fiji software (Schindelin et al., 2012). The images were first adjusted for brightness and contrast. Images from different channels were merged together and the maximal projection of the images was obtained.

\subsubsection{Behavioral paradigms}

\subsubsection{Paralysis paradigm}

The flies expressing the heat-activated channel dTRPA1 (Hamada et al., 2008) under control of the actin promoter were raised at $18^{\circ} \mathrm{C}$. 5-6 days old flies were placed in small $(27 \times 64 \mathrm{~mm})$ plastic vials ( $1 \mathrm{fly}$ per vial). The vials were kept at $32^{\circ} \mathrm{C}$ in a water-bath and the time until the flies got paralysis was measured. The time measurement was stopped after 4 min.

\subsubsection{Single fly assay for walking velocity}

The apparatus consists of an aluminum plate $(30 \times 11 \times 0.4 \mathrm{~cm})$ placed on the plastic base. The plate was divided into 10 fly "run arenas" $(29 \times 0.5 \times 0.4 \mathrm{~cm})$ by aluminum borders and covered with Plexiglas. The aluminum plate was covered with white paper to obtain a high contrast between the background and the flies. For controlling the temperature in the apparatus, it was placed in an incubator with a cooling device. An external heater was placed in the incubator to achieve an adjusted temperature of $26^{\circ} \mathrm{C}$ to $32^{\circ} \mathrm{C}$. The temperature in the apparatus was measured by placing the wire of a digital thermometer in one of the arenas. For each behavioral experiment the velocity of 9 flies was measured simultaneously. The flies, without being anesthetized, were transferred to the arenas and the activity was recorded for $10 \mathrm{~min}$ using a camera (DCR-SR57, Sony) installed at the top of the apparatus. The experiment was performed under constant red light condition produced by LEDs installed at the walls of the incubator. The humidity in the incubator was maintained between 55\% and $65 \%$ (Figure 2.1). The movies acquired during the experiments were then analyzed using the video tracking software, Noldus EthoVision XT 8.5. 
A

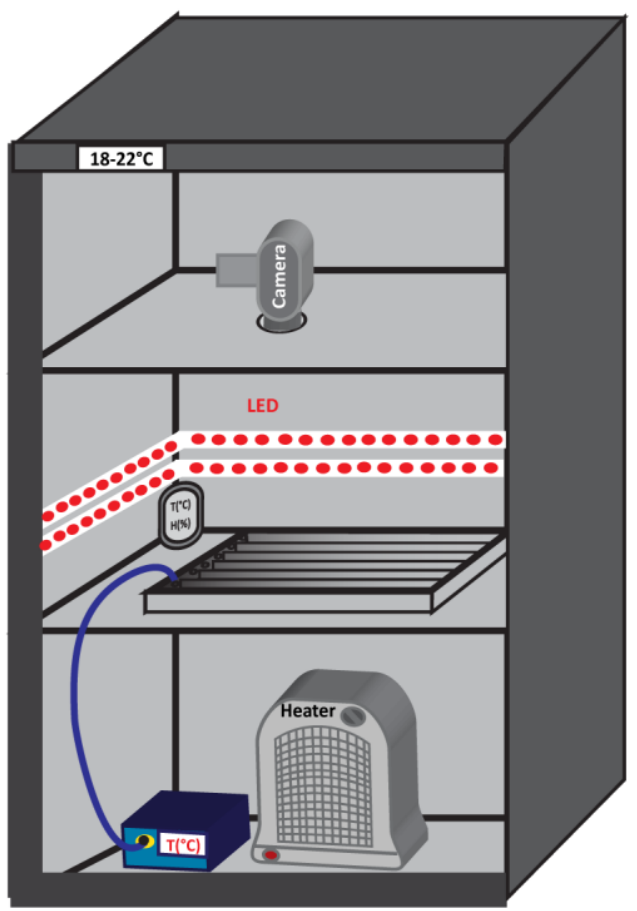

B

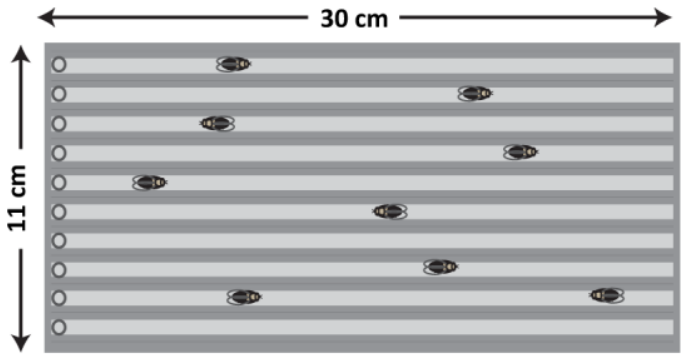

Figure 2.1. Scheme of the walking velocity apparatus. (A) For controlling the temperature in the walking velocity apparatus, the it was placed in an incubator with a cooling device (for temperature between $18-22^{\circ} \mathrm{C}$ ). An external heater was placed in the incubator to achieve an adjusted temperature of $26^{\circ} \mathrm{C}$ to $32^{\circ} \mathrm{C}$. The temperature in the apparatus was measured by placing the wire of a digital thermometer in one of the arenas. The flies' activity was recorded for 10 min using a camera installed at the top of the apparatus. The experiment was performed under constant red light condition produced by LEDs installed at the walls of the incubator. (B) Top view of the walking velocity set up which was divided into 10 fly "run arenas".

\subsubsection{Negative geotaxis}

For measuring the negative geotaxis two assays were used:

Assay 1: A $10 \mathrm{ml}$ serological pipette of $28 \mathrm{~cm}$ length was divided into three zones (top: $7 \mathrm{~cm}$, middle: $14 \mathrm{~cm}$ and bottom: $7 \mathrm{~cm}$ ). The tip of the pipette was sealed using parafilm. Flies were kept in small food vials (10 female flies per vials) after eclosion. 5-6 days old flies were transferred to the pipette without any anesthesia. This experiment was performed at $18^{\circ} \mathrm{C}$ and $32^{\circ} \mathrm{C}$. After 10 min resting time, the pipette was tapped on the ground to agitate the flies. After 1 min, number of flies in each compartment was counted. After additional 5 min of resting, the pipette was tapped again and number of the flies was counted (Figure $2.2 \mathrm{~A}$ ). For each pipette this procedure was repeated three times (Friggi-Grelin et al., 2003). The negative geotaxis score was calculated using the following formula: 
Negative geotaxis score $_{\text {(assay 1) }}=1 / 2 \times\left[\mathrm{N}_{\text {(total) }}+\mathrm{N}_{\text {(up) }}-\mathrm{N}_{\text {(down) }} / \mathrm{N}_{\text {(total) }}\right]$

The average of the negative geotaxis score for each pipette was counted as one sample size.

Assay 2: This negative geotaxis assay modified from Benzer paradigm (Benzer, 1976) was described by Inagaki et al. (2009). In this assay the apparatus consists of two frames with the sliding rail in between. Six tubes were attached to the lower frame and five tubes to the upper frame. The transferring and the testing states can be produced by sliding the two frames. In the transferring state 20 flies (5-6 days old) were placed in the first "down tube". The apparatus was tapped 5 times to the bench to agitate the flies. By a quick sliding of the upper frame the transferring state was changed to the testing state and the flies had $30 \mathrm{~s}$ to climb to the first upper tube. After 30 seconds the upper frame was slid back to the transferring state and the apparatus was tapped again 5 times to the bench. The flies that climbed up to the first upper tube were transferred to the bottom of the second lower tube. This sequence was repeated for four more times. At the end the flies were distributed in the six tubes and they had five times chances to climb up or stay at the bottom (Figure $2.2 \mathrm{~B}$ ). This test was performed at $18^{\circ} \mathrm{C}$ and $32^{\circ} \mathrm{C}$. The negative geotaxis score was calculated using the following formula:

Negative geotaxis score $_{(\text {assay 2) }}=1 / 5 \times\left(N_{2}+2 N_{3}+3 N_{4}+4 N_{5}+5 N_{6}\right) / N_{1}+N_{2}+N_{3}+N_{4}+N_{5}+N_{6}$

A

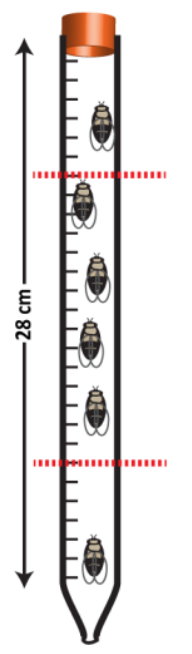

B

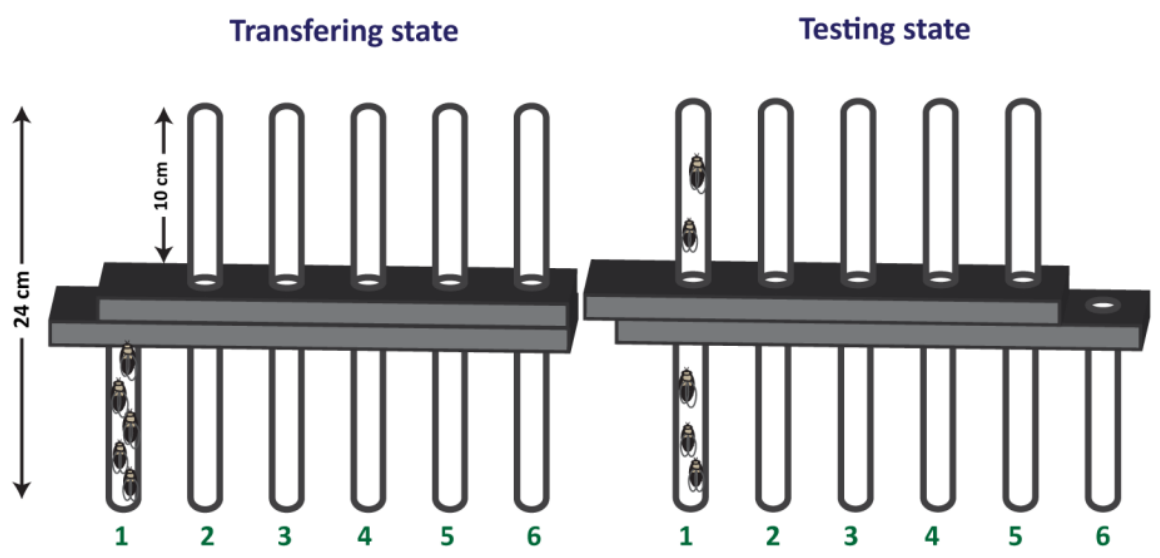

Figure 2.2. Scheme of the negative geotaxis assays. (A) Assay 1: a $10 \mathrm{ml}$ serological pipette of $28 \mathrm{~cm}$ length was divided into three zones (top: $7 \mathrm{~cm}$, middle: $14 \mathrm{~cm}$ and bottom: $7 \mathrm{~cm}$ ). The tip of the pipette was sealed using parafilm. (B) Assay 2: the apparatus consists of two frames with the sliding rail in between. Six tubes were attached to the lower frame and five tubes to the upper frame. The transferring and the testing states can be produced by sliding the two frames. 


\subsubsection{Shock avoidance}

Shock avoidance was measured in a T-maze consisting of a "shock tube" and a "safe tube". The shock tube consisted of copper grids. For the experiment, 50 to 60 flies were placed in the shock tube and 12 electric shocks of $90 \mathrm{~V}$ (1.25 second shock with 3.75 second inter pulse interval) was applied. The flies had 1 min to escape from the shock tube and go to the safe tube (Figure 2.3). The experiment was performed at either $22^{\circ} \mathrm{C}$ or $32^{\circ} \mathrm{C}$. After counting the flies on each side of the T-maze the shock avoidance index was calculated using the following formula:

Shock avoidance index $=$ (Number of flies in the safe tube $/$ total number of flies $) \times 100$

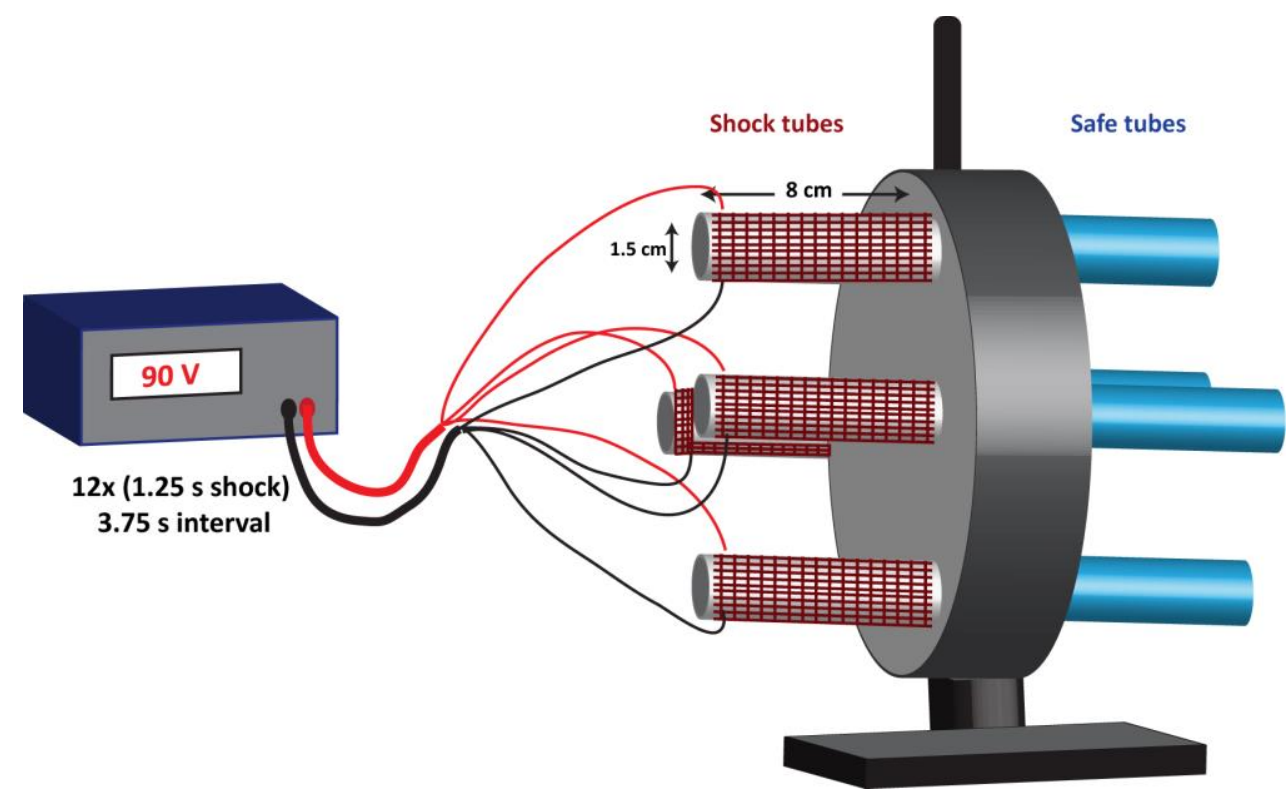

Figure 2.3. Scheme of the shock avoidance apparatus. The T-maze apparatus consisted of a "shock tube" and a "safe tube". The shock tubes consisted of copper grids. For the experiment, in the shock tube 12 electric shocks of $90 \mathrm{~V}$ (1.25 second shock with 3.75 second inter pulse interval) was applied.

\subsubsection{Flight assay}

Groups of 100 flies were dropped into a $500 \mathrm{ml}$ cylinder (of $50 \mathrm{~mm}$ diameter) using a funnel. The wall of the cylinder was coated with paraffin oil. To avoid falling to the ground, the fly had to fly and got trapped in the oil coating the container (Figure 2.4). In order to quantify the flight index, the cylinder was divided into two halves and the index was calculated using the following formula (Wagh et al., 2006):

Flight index $=$ (Number of flies in the upper half/ total number of flies) $\times 100$ 
The experiment was performed at either $22^{\circ} \mathrm{C}$ or $32^{\circ} \mathrm{C}$.

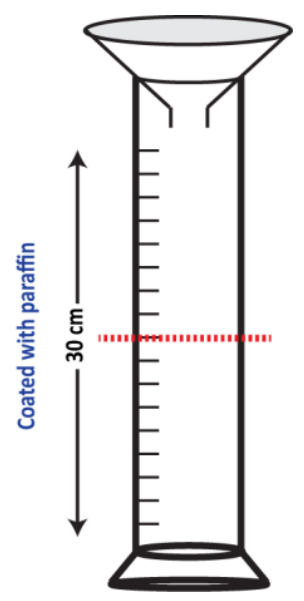

Figure 2.4. Scheme of the flight assay. The wall of the $500 \mathrm{ml}$ cylinder (of $50 \mathrm{~mm}$ diameter) was coated with paraffin oil. Groups of 100 flies were dropped into the cylinder using a funnel.

\subsubsection{Feeding}

For feeding experiments flies were starved for 2 days at $18^{\circ} \mathrm{C}$. For starvation 25 female flies were placed in a medium vial with a moist tissue paper with a tap water at the bottom of the vial. After starvation flies were transferred to the food vials containing red dye or control vials (section 2.1.8.3) without anesthesia for $45 \mathrm{~min}$ at $18^{\circ} \mathrm{C}$ or at $32^{\circ} \mathrm{C}$. Afterwards, flies were anesthetized and kept on ice. 20 flies of each tested genotype were decapitated and the bodies were collected in $2 \mathrm{ml}$ micro-centrifuge tubes containing a steel bead ( $5 \mathrm{~mm}$ diameter). $500 \mu \mathrm{l}$ of tap water was added to each tube. Homogenization of the tissue was done using a Tissuelyser for $10 \mathrm{~min}$ at $50 \mathrm{~Hz}$. The tubes were then centrifuged for $1 \mathrm{~min}$ at $13000 \mathrm{rpm}$. The readout for quantification of red dye was performed using an Epoch Microplate Spectrophotometer. In a 96-well plate, each well was filled with $100 \mu \mathrm{l}$ of the supernatant. Correcting for possible pipetting errors, the readout for each sample was taken three times. For quantification, the absorbance of the red dye was measured at $500 \mathrm{~nm}$. For each genotype, the mean absorbance at $500 \mathrm{~nm}$ of the control flies (fed on the white food) was deducted from the absorbance of the test flies (fed on the red food) to make sure that the source of the red dye absorbance was the red food. 


\subsubsection{Activity and sleep measurements}

Using the Drosophila Activity Monitoring (DAM) system, the movement of the flies was measured over time. The protocol for using this system for monitoring locomotion activity was described by Pfeiffenberger et.al (2010). Individual flies were placed in the glass tubes sealed with fly food and a plastic cap on one side and a cotton plug on the other side. Each Activity Monitor has 32 holes for inserting the tubes containing a single fly. An infrared beam in the middle of each tube measures the activity by counting the number of times a fly crosses the beam. The activity is recorded for each fly in the given interval time. The loaded activity monitors were placed in a small incubator with $12 / 12 \mathrm{~h}$ the light/dark cycles. The monitors in the incubator were connected to the DAM system network for providing a readout of the activity using DAMFileScan Software. Using this software the output file of the activity reading was obtained as text files. For this assay, anesthetized 3 days old females were placed in the tubes. Tubes were first kept for one day at $25^{\circ} \mathrm{C}$ (raising temperature) for the recovery from anesthesia and getting used to the new environment. The experiments were performed in $12 \mathrm{~h}$ light-dark cycles at $22^{\circ} \mathrm{C}$ or $32^{\circ} \mathrm{C}$ for 3 days. The interval time between readouts was $1 \mathrm{~min}$. The output data were analyzed using an Excel Visual Basic Application (VBA) script written by Shubham Dipt. Activity trace and sleep trace for each $30 \mathrm{~min}$, total activity per day and night, total sleep per day and night were calculated from the output text files. The criterion for the sleep was assigned as immobility for at least 5 min (Shaw et al., 2000; Nitz et al., 2002).

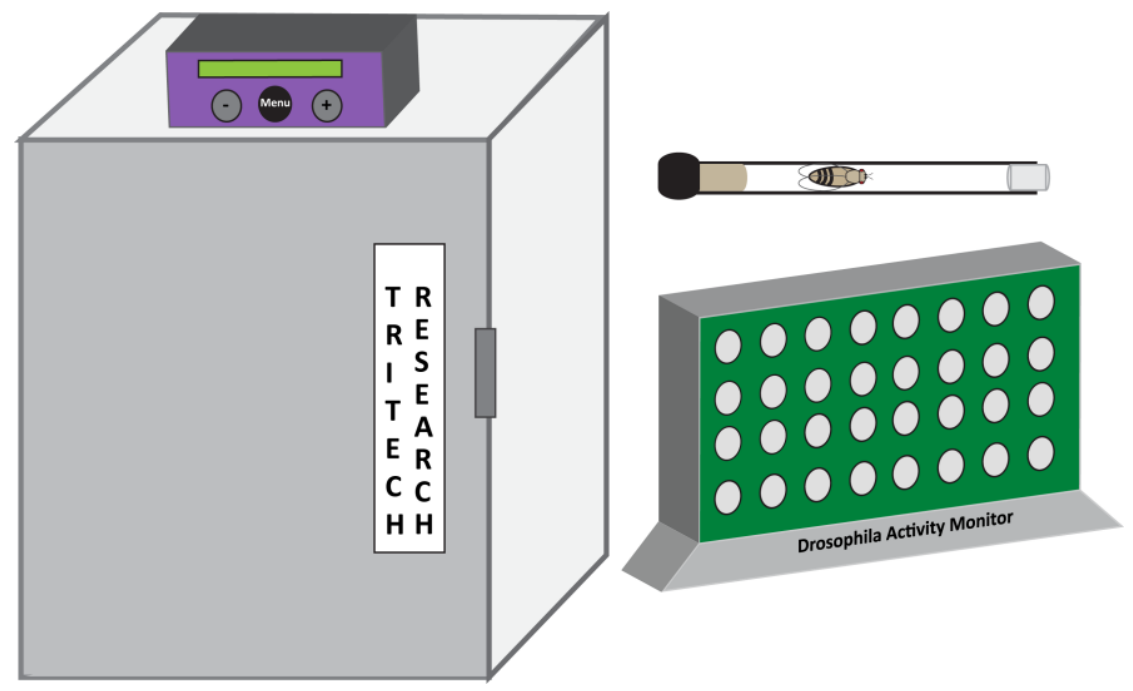

Figure 2.5. Scheme of the Drosophila Activity Monitoring (DAM) system. Individual flies were placed inside the glass tubes sealed with fly food and a plastic cap on one side and a cotton plug on the other side. Each Activity Monitor had 32 holes for inserting the tubes containing a single fly. An infrared beam crossing the middle of each 
tube measured the activity by counting the number of times a fly crosses the beam. The activity monitors were placed inside a small incubator with $12 / 12 \mathrm{~h}$ the light/dark cycles.

\subsubsection{Drug experiment}

For reduction of the serotonin synthesis, flies were fed with the para-chlorophenylalanine (PCPA) drug (Koe and Weissman, 1966). 3-4 days female flies were starved for 2 days at $18^{\circ} \mathrm{C}$ and transferred to the PCPA food or control food (section 2.1.8.2) for 4 days at $25^{\circ} \mathrm{C}$. The reduction in the serotonin level was not observed when the flies were kept at $18^{\circ} \mathrm{C}$ during the drug treatment. The quantification of the serotonin reduction was done by immunostaining against serotonin (5-HT) in the Conton-S flies.

\subsubsection{Statistical analysis}

Statistical analysis was performed using the Origin $8.5 \mathrm{G}$ software. Data were tested for normal distribution using Shapiro-Wilk normality test. For testing statistically significant differences between two groups, Mann-Whitney test (non-parametric test) or two-sample t-test and for comparisons between more than two groups, Kruskal-Wallis Anova (non-parametric test) or one-way ANOVA with post hoc Bonferroni correction were used. 


\section{Results}

\subsection{The distribution of serotoninergic cells in the Drosophila brain}

In this study, using an antibody against serotonin (5-hydroxytryptophane, 5-HT), 91 \pm 13 (mean $\pm S D$ ), serotoninergic neurons in the brains of adult, female flies were stained and the relative positions of their somata determined. As described by other studies (Valles and White, 1988; Sitaraman et al., 2008; Alekseyenko et al., 2010; Pech et al., 2013), the cell bodies are distributed in spatially distinct clusters (Figure 3.1). The results of this study were compared with previous studies that describe the clusters with differing names and to different degrees in detail (Valles and White, 1988; Sitaraman et al., 2008; Alekseyenko et al., 2010; Pech et al., 2013). To clarify these differences, a modified nomenclature for serotoninergic neurons based on the location of the somata in the brain is introduced here (Figure 3.1; Table 3.1). Furthermore, the present study identifies a serotoninergic cluster that consists of two anterior dorsal-medial located paired somata (ADMP) which have not been described by previous studies (Figure 3.1; Table 3.1).

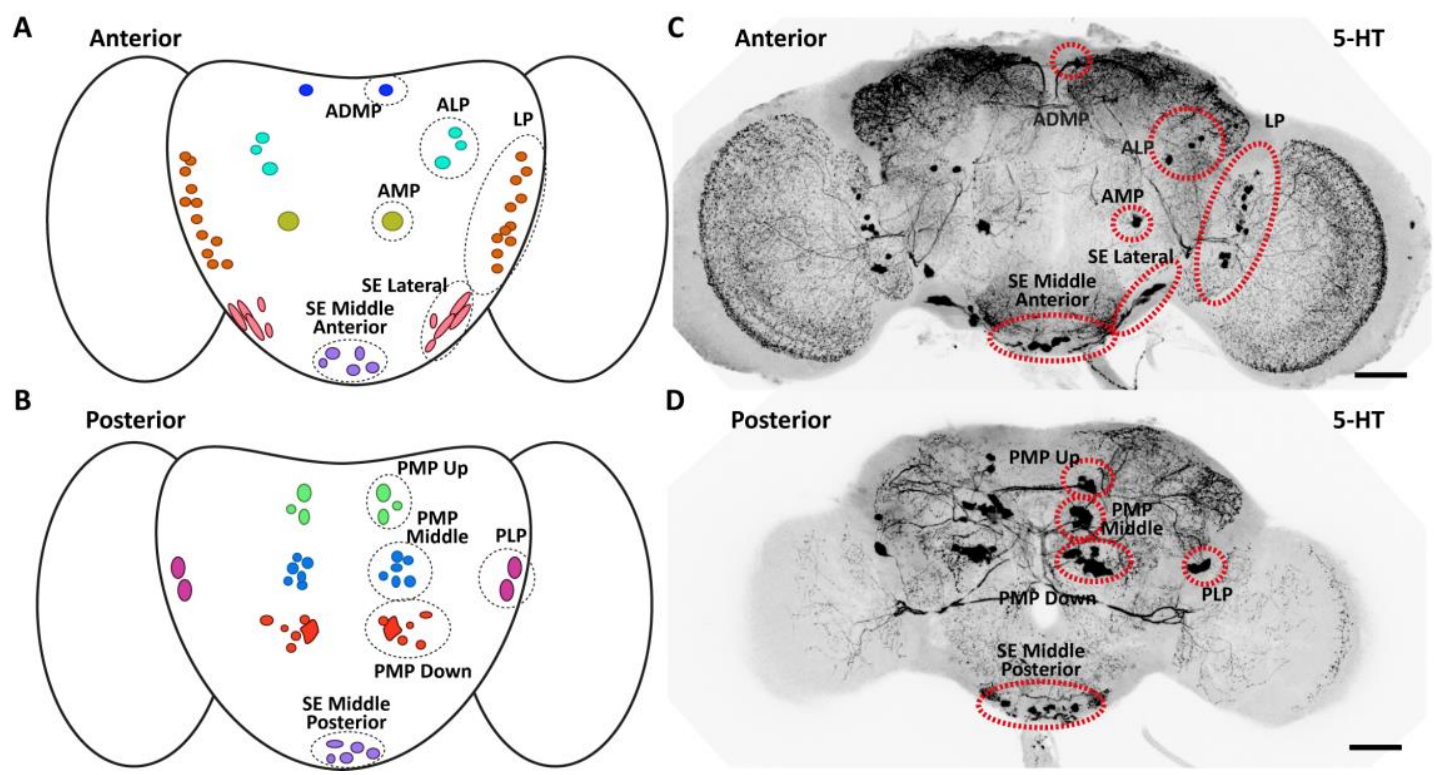

Figure 3.1. Distribution of serotoninergic cell clusters in the adult Drosophila brain. (A, B) Schematic illustration of the anterior and posterior views of the Drosophila brain and the localization pattern of the serotoninergic clusters in the anterior (ADMP, ALP, LP, SE lateral and SE middle anterior) and the posterior (PLP, SE middle 
posterior, PMP UP, PMP middle and PMP down) regions of the brain. (C, D) Immunohistochemical staining against serotonin (5-HT) in the Drosophila brain. The distribution of the anti-serotonin immunoreactive somata and their innervation patterns are shown in the anterior and posterior views of the brain.

Table 3.1. Nomenclature and position of the serotoninergic clusters in the Drosophila brain. The number of antiserotonin-immunoreactive somata in each cluster was counted ( $n=18$ entire brains, both hemispheres). The mean of the cell number \pm SD in each cluster per brain (both hemispheres), the exact location of the clusters and also the nomenclature of serotoninergic clusters used in previous studies are indicated here.

\begin{tabular}{|c|c|c|c|c|}
\hline $\begin{array}{l}\text { 5-HT } \\
\text { clusters }\end{array}$ & $\begin{array}{l}\text { No. of somata / } \\
\text { brain }(n=18)\end{array}$ & Location & $\begin{array}{l}\text { Valles and White's } \\
\text { nomenclature }\end{array}$ & $\begin{array}{l}\text { Sitaraman's } \\
\text { nomenclature }\end{array}$ \\
\hline ALP & $6 \pm 0$ & $\begin{array}{l}\text { Anterior lateral } \\
\text { protocerebrum }\end{array}$ & - & ALP \\
\hline AMP & $2 \pm 0$ & $\begin{array}{l}\text { Anterior medial } \\
\text { protocerebrum }\end{array}$ & - & AMP \\
\hline ADMP & $2 \pm 0$ & $\begin{array}{l}\text { Anterior dorsomedial } \\
\text { protocerebrum }\end{array}$ & - & - \\
\hline LP & $24 \pm 3$ & $\begin{array}{l}\text { lateral } \\
\text { protocerebrum }\end{array}$ & LP2a,b & LP2 \\
\hline SE Lateral & $10 \pm 2$ & $\begin{array}{l}\text { Lateral } \\
\text { subesophageal }\end{array}$ & SE1 and SE2 & SE1 and SE2 \\
\hline SE Middle* & $10 \pm 3$ & $\begin{array}{l}\text { Subesophageal } \\
\text { midline }\end{array}$ & SE3 & SE3 \\
\hline PLP & $4 \pm 0$ & $\begin{array}{l}\text { Posterior lateral } \\
\text { protocerebrum }\end{array}$ & LP1 & PLP \\
\hline PMP up & $6 \pm 0$ & $\begin{array}{l}\text { posterior medial } \\
\text { protocerebrum up }\end{array}$ & SP1 & PMP \\
\hline PMP middle & $13 \pm 2$ & $\begin{array}{l}\text { posterior medial } \\
\text { protocerebrum middle }\end{array}$ & SP2 & PMP \\
\hline PMP down & $14 \pm 3$ & $\begin{array}{l}\text { posterior medial } \\
\text { protocerebrum down }\end{array}$ & IP & PMP \\
\hline
\end{tabular}

\section{Sum $\quad 91 \pm 13$}

* Due to the difficulty in distinction between the SE middle anterior and SE middle posterior clusters in some cases, the cells in these two clusters are merged and indicated as SE middle cluster. 


\subsection{Tools to manipulate the activity of serotoninergic neurons}

In order to investigate the role of serotonin in the modulation of the arousal level in Drosophila, a thermogenetic approach was used for remote activation of serotoninergic neurons. Using the UAS-Gal4 system (section 1.3.1.2) the heat-activated channel, dTRPA1 (Hamada et al., 2008), was expressed in the serotoninergic neurons and the effect of their neuronal activity was investigated in different behavioral paradigms in the context of the animals' arousal state. In order to visualize the expression pattern of the dTRPA1 channel in the brain, it was coupled to the red fluorescent protein mCherry and a new transgenic Drosophila line carrying this construct behind a UAS sequence was generated. For expressing the mCherry-coupled dTRPA1 protein in serotoninergic neurons, three different Gal4 lines were available (provided kindly by Dr. Jaeseob Kim, Dr. Serge Birman and Dr. Edward Kravitz). In all three lines, Gal4 expression is controlled by a tyrosine hydroxylase promoter (TRH). Therefore, the UAS-driven gene expression is expected to be restricted to serotonin-producing cells. In the following sections, the results of generating and characterizing the transgenic lines for the mCherry tagged dTRPA1 channel are described, followed by a comparison of the three available TRH-Gal4 lines.

\subsubsection{Generating a tool for thermogenetic neuronal activation}

In order to activate a specific subset of neurons, the heat-activated channel dTRPA1 (Hamada et al., 2008) was used as a thermogenetic tool. The dTRPA1 channel has temperaturedependent excitability and responds to temperature higher than $27^{\circ} \mathrm{C}$ (Viswanath et al., 2003). In this study, for visualizing and tracking the expression of dTRPA1, this channel was tagged with a red fluorescent protein (mCherry) (Lefkimmiatis et al., 2009), fused either to the Nterminus or C-terminus of the protein. Using the UAS-Gal4 binary system (Brand and Perrimon, 1993) the UAS:tagged dTRPA1 DNA construct was expressed under control of different Gal4 driver lines.

\section{Functional tagged dTRPA1 channel}

Because it was not known whether a tag would influence the functionality of the dTRPA1 channel with respect to temperature sensitivity and conductivity, two versions of mCherrytagged channels were generated ( $\mathrm{N}$-terminal or $\mathrm{C}$-terminal). Due to the random insertion of the P-element into the fly genome, five different fly strains with various insertion sites were 
received from the BestGene Inc. company that performed the germline transformation (Table 3.2).

Table 3.2. Overview of the generated UAS:dTRPA1-mCherry and UAS:mCherry-dTRPA1 lines. The chromosome carrying the UAS construct is indicated for each line. The asterisk indicates the UAS line used for the anatomical and behavioral studies.

\begin{tabular}{|c|c|c|c|c|}
\hline Tagged $d T R P A 1$ lines & $\begin{array}{l}\text { mCherry } \\
\text { tag }\end{array}$ & Chromosome & $\begin{array}{l}\text { Channel } \\
\text { functionality }\end{array}$ & $\begin{array}{l}\text { mCherry } \\
\text { fluorescence }\end{array}$ \\
\hline w; UAS:dTRPA1-mCherry; + (1) & C-terminal & 2nd & + & + \\
\hline$w ;++$ UAS:dTRPA1-mCherry (2) & C-terminal & $3 r d$ & - & + \\
\hline w, UAS:dTRPA1-mCherry; +; + (3)* & C-terminal & $x$ & + & + \\
\hline$w^{-} ;+$; UAS:mCherry-dTRPA1 (1) & N-terminal & $3 r d$ & + & + \\
\hline$w ;+;$ UAS:mCherry-dTRPA1 (2) & N-terminal & $3 r d$ & + & + \\
\hline
\end{tabular}

For determining the functionality of the tagged dTRPA1 channel, the dTRPA1-mCherry or mCherry-dTRPA1 was expressed globally in the fly using an actin-Gal4 driver line (Funk et al., 2004). The actin>tagged dTRPA1 flies were tested for temperature-dependent paralysis (section 2.2.5.1). Four out of five lines showed paralysis at $32^{\circ} \mathrm{C}$, which is caused by a dTRPA1-mediated depolarization of the muscles and neurons (Table 3.2; Figure $3.2 \mathrm{~A}$ ). Furthermore, all of the actin>tagged dTRPA1 flies expressed mCherry in their entire body (Figure 3.2 D). For a quantification of mCherry expression in the different strains, the fluorescence intensity of the mCherry protein was measured through the cuticle in the antenna (Figure 3.2.E; section 2.2.4.1). Although the expression of the mCherry protein was detected in all of the fly lines, one strain showing no paralysis had the lowest intensity of mCherry fluorescence (Figure 3.2 B). Based on the result of this experiment, both versions of dTRPA1 channel with N-terminal and Cterminal mCherry tagged were found to be functional, but the efficiency depended on the particular insertion site of the construct. When comparing different strains, both the UAS:mCherry-dTRPA1 (2) and the UAS:dTRPA1-mCherry (3) line had a functional dTRPA1 channel while showing higher fluorescence intensity than others (Figure 3.2 C). 
For selecting the best line for the behavioral and anatomical experiments, the parental UAS lines were tested for a potential leakiness of transgene expression (i.e., gene expression without the presence of any Gal4 protein). The fresh brains dissected in the Ringer's solution, were mounted in the vectashield and were scanned using confocal microscope. In the UAS:dTRPA1-mCherry (3) line no mCherry expression was observed. Therefore, this line was used for the thermogenetic activation of the neurons in the following experiments.

A

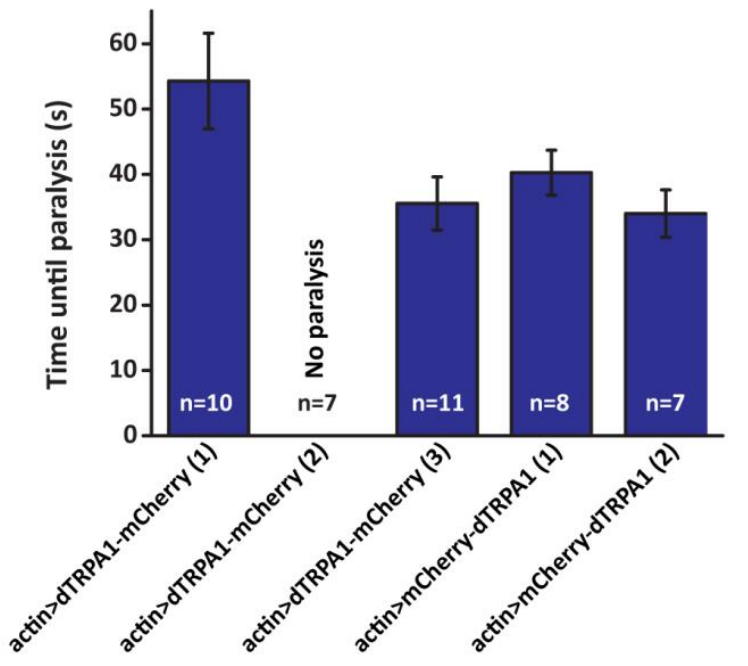

C

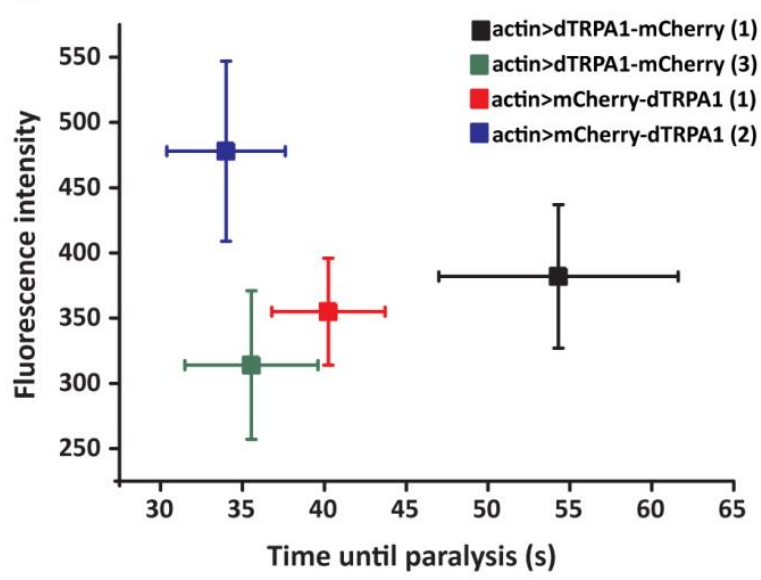

B

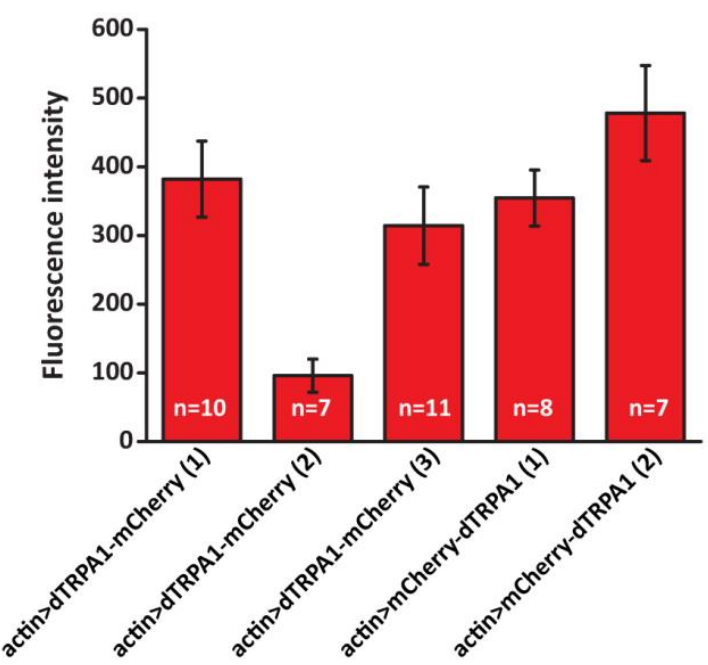

D

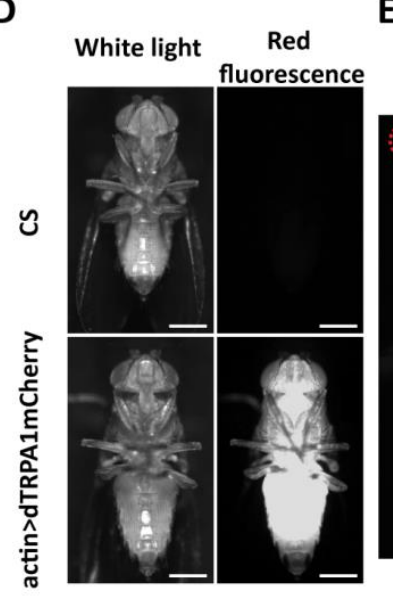

$\mathbf{E}$

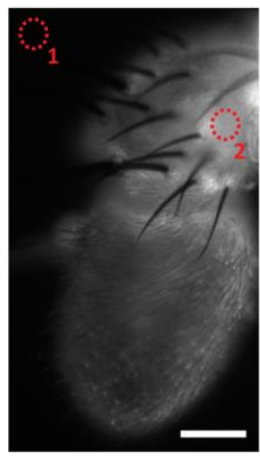

Figure 3.2. Characterization of fly strains expressing the mCherry-tagged dTRPA1 channel. (A) The flies expressing different versions of the dTRPA1 under the control of actin promoter were tested in a paralysis paradigm. The time until paralysis at $32^{\circ} \mathrm{C}$ was measured for each line. The actin>dTRPA1-mCherry (2) line did not show any paralysis within the 3 minutes observation time. The sample size is indicated in the graph. (B) In the actin>mCherry-tagged dTRPA1, fluorescence intensity of the mCherry protein was measured in the antenna. The sample size is indicated in the graph. (C) For selecting the fly strain with high mCherry fluorescence and functional dTRPA1 channel, the 
fluorescence intensity and the time until paralysis parameters were plotted. The time until paralysis parameter which indicates the functionality of the channel was lower in actin>mCherry-dTRPA1 (2) and actin>dTRPA1mCherry (3) lines. But the mCherry expression was higher in actin>mCherry-dTRPA1 (2) line. (D) The red fluorescence expression in actin>mCherry-dTRPA1 line and CS fly (as a negative control) is illustrated. Scale bar: $500 \mu \mathrm{m}$. (E) The antenna of the fly was used for quantification of mCherry fluorescence. The fluorescence intensity was measured in the region 1 and 2 . The final fluorescence intensity was obtained by reduction of background fluorescence (region 1) from the fluorescence intensity in the antenna (region 2). Dots indicate means, errors bars indicate standard error of the mean. Scale bar: $50 \mu \mathrm{m}$.

\subsubsection{Anatomical comparison of the expression pattern induced by three tryptophan hydroxylase driver lines (TRH-Gal4)}

For expressing the transgenes in serotoninergic neurons, three Gal4 lines, TRH-Gal4 (1), TRHGal4 (2) and TRH-Gal4 (3) provided kindly by Dr. Kim, Dr. Birman and Dr. Kravitz, respectively, were available (Park et al., 2006a; Sitaraman et al., 2012; Alekseyenko et al., 2010). For visualizing and comparing the expression pattern of these Gal4 lines, UAS:mCD8-GFP (Pfeiffer et al., 2010) line was used as a fluorescence reporter. For each Gal4 line, the number of serotoninergic ("specific") and the non-serotoninergic ("unspecific") cells were determined by anti-GFP and anti-5-HT co-staining (Table 3.3; Figure 3.3 C, D, G, H, K, L). These TRH-Gal4 lines covered different amount of serotoninergic cells and expressed in different amount of unspecific cells. TRH-Gal4 (2) (Sitaraman et al., 2012) labels the most specific cells while labeling the lowest amount of unspecific cells. Conversely, the TRH-Gal4 (1) (Park et al., 2006a) targeted the lowest amount of serotoninergic cells.

To determine any effect of the reporter line efficiency on the expression pattern of specific and unspecific cells in TRH-Gal4 lines, the newly generated UAS:dTRPA1-mCherry line was used and the expression compared to the one obtained with UAS:mCD8-GFP. A double-staining against mCherry and serotonin was performed using anti-RFP and anti-5-HT and the expression pattern of each line was described (Table 3.3; Figure 3.3 A, B, E, F, I, J). The evaluation indicates a higher number of TRH-Gal4-labeled neurons in TRH $>$ dTRPA1-mCherry flies when compared to TRH>mCD8-GFP flies. The observed differences were more obvious in the number of nonserotoninergic neurons (Table 3.3). The dependency of the expression strength on the UAS:reporter used has been described (Thum et al., 2006). 
Table 3.3. Number of the serotoninergic cells showing gene expression induced by TRH-Gal4 fly lines. The number of serotoninergic cells and non-serotoninergic cells (unspecific) in each cluster covered by TRH-Gal4 (1), TRH-Gal4 (2) and TRH-Gal4 (3) were counted (mean \pm SD). For each Gal4 line, two UAS lines, UAS:mCD8-GFP (referred to as GFP) and UAS:dTRPA1-mCherry (referred to as mCherry) were used for characterization ( $n=3$ entire brains, both hemispheres The number of cells in each cluster indicates the cell number in both hemispheres.

\begin{tabular}{|c|c|c|c|c|c|c|}
\hline \multirow{2}{*}{$\begin{array}{l}\text { 5-HT } \\
\text { clusters }\end{array}$} & \multicolumn{2}{|c|}{ TRH-Gal4 (1) } & \multicolumn{2}{|c|}{ TRH-Gal4 (2) } & \multicolumn{2}{|c|}{ TRH-Gal4 (3) } \\
\hline & GFP & mCherry & GFP & mCherry & GFP & mCherry \\
\hline ALP & $0 \pm 0$ & $2 \pm 0$ & $5 \pm 1$ & $4 \pm 1$ & $4 \pm 0$ & $4 \pm 1$ \\
\hline AMP & $0 \pm 0$ & $0 \pm 0$ & $2 \pm 0$ & $2 \pm 0$ & $1 \pm 1$ & $2 \pm 0$ \\
\hline ADMP & $0 \pm 0$ & $0 \pm 0$ & $0 \pm 0$ & $2 \pm 0$ & $1 \pm 1$ & $2 \pm 0$ \\
\hline LP & $6 \pm 1$ & $11 \pm 1$ & $14 \pm 2$ & $19 \pm 3$ & $24 \pm 2$ & $18 \pm 3$ \\
\hline SE Lateral & $3 \pm 1$ & $5 \pm 1$ & $11 \pm 1$ & $12 \pm 0$ & $12 \pm 3$ & $11 \pm 1$ \\
\hline SE Middle & $9 \pm 4$ & $8 \pm 2$ & $7 \pm 1$ & $8 \pm 3$ & $7 \pm 2$ & $9 \pm 3$ \\
\hline PLP & $3 \pm 1$ & $4 \pm 0$ & $4 \pm 0$ & $4 \pm 0$ & $4 \pm 1$ & $4 \pm 0$ \\
\hline PMP up & $4 \pm 0$ & $4 \pm 0$ & $6 \pm 0$ & $6 \pm 0$ & $6 \pm 0$ & $6 \pm 0$ \\
\hline PMP middle & $3 \pm 1$ & $5 \pm 2$ & $10 \pm 1$ & $10 \pm 3$ & $7 \pm 1$ & $12 \pm 3$ \\
\hline PMP down & $9 \pm 2$ & $9 \pm 3$ & $9 \pm 3$ & $10 \pm 2$ & $10 \pm 2$ & $8 \pm 3$ \\
\hline Sum (specific) & $37 \pm 10$ & $48 \pm 9$ & $68 \pm 9$ & $79 \pm 12$ & $76 \pm 13$ & $76 \pm 14$ \\
\hline Unspecific & $113 \pm 10$ & $233 \pm 24$ & $115 \pm 10$ & $168 \pm 12$ & $124 \pm 14$ & $198 \pm 22$ \\
\hline
\end{tabular}




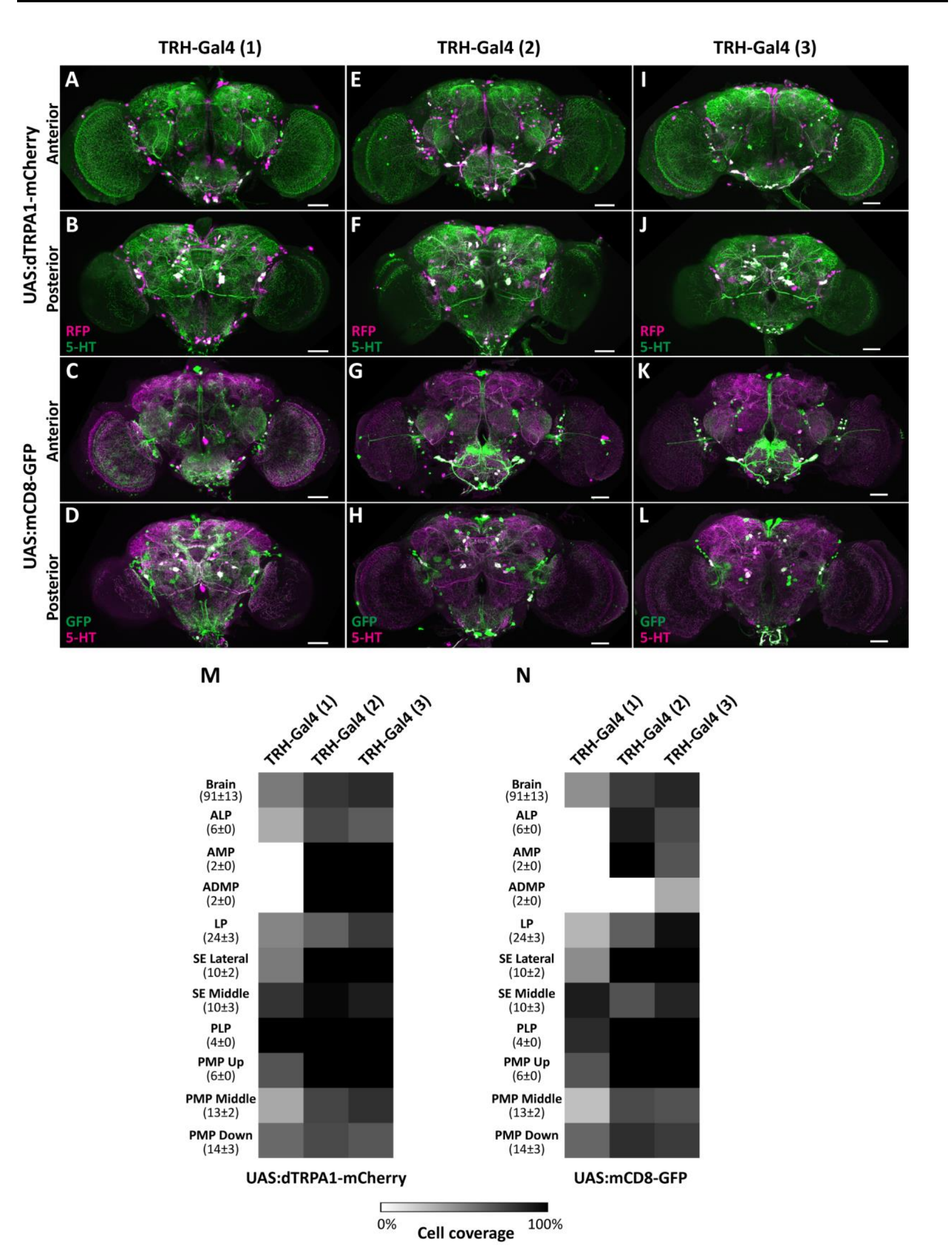


Figure 3.3. Characterization of TRH-Gal4 (1), TRH-Gal4 (2) and TRH-Gal4 (3) lines. (A, B, E, F, I, J) The anterior and posterior views of the Drosophila brains expressed dTRPA1-mCherry under control of three TRH-Gal4 lines. The expression pattern was visualized using an anti-RFP antibody labelling the mCherry protein (magenta) and an anti5-HT antibody (green). The staining in white color indicates the serotoninergic cells covered by each Gal4 line. (C, D, G, H, K, L) The anterior and posterior views of the Drosophila brains expressed mCD8-GFP under the control of the TRH-Gal4 lines. The expression pattern was visualized using an anti-GFP antibody (green) and an anti-5-HT antibody (magenta). The co-staining of the anti-5-HT and anti-GFP antibodies (white) indicates the serotoninergic cells covered by each Gal4 line. (M, N) The percentage of the serotoninergic neurons covered by each TRH-Gal4 lines in the entire brain and in each cluster (both hemispheres) is illustrated ( $n=3$ entire brains). The white and black colors indicate $0 \%$ and $100 \%$ serotoninergic cell coverage, respectively. The numbers in parentheses (mean $\pm S D$ ) show the number of anti-serotonin immunoreactive cells in the entire brain and in each cluster (both hemispheres).

\subsection{Effect of thermogenetically activating serotoninergic neurons on the arousal state in Drosophila}

Since the locomotor activity is a common indicator for measuring the arousal level in Drosophila research (Shaw et al., 2000; Andretic et al., 2005), different behavioral paradigms for investigating the walking velocity and responsiveness to different external stimulus were designed. Using these paradigms, the possible role of serotoninergic neurons in regulating the arousal level in flies was investigated.

\subsubsection{Thermogenetic activation of serotoninergic neurons leads to a significant reduction in the walking velocity}

The UAS:dTRPA1-mCherry line was crossed to TRH-Gal4 (1), TRH-Gal4 (2) and TRH-Gal4 (3), which allowed for thermogenetically activating serotoninergic neurons in the respective offspring. In order to investigate a possible effect of activating serotoninergic neurons on the walking velocity of the flies, a single fly assay for walking velocity (section 2.2.5.2) was used. The walking velocity of the flies (the offspring of each cross as the test group and the heterozygous parental lines as control groups) was measured at six different temperatures $\left(18^{\circ} \mathrm{C}, 23^{\circ} \mathrm{C}, 26^{\circ} \mathrm{C}\right.$, $28^{\circ} \mathrm{C}, 30^{\circ} \mathrm{C}$ and $32^{\circ} \mathrm{C}$ ). At the first three temperatures, which do not activate dTRPA1, no difference between test and control groups was observed. At temperatures from $28^{\circ} \mathrm{C}$ to $32^{\circ} \mathrm{C}$ the control flies walked faster than at colder temperatures, but the walking velocity of the test flies with thermogenetically activated serotoninergic neurons was drastically reduced (Figure 
3.4). This is reflected by a highly significant difference in walking velocity when comparing control and test flies at temperatures above $28^{\circ} \mathrm{C}$. Based on this result, it was deducted that the reduction in the velocity was due to the activation of serotoninergic neurons, because TRPmediated activation is directly temperature-dependent.

A

A 1 M TRH-Gal4 (1)
UAS:dTRPA1-mCherry

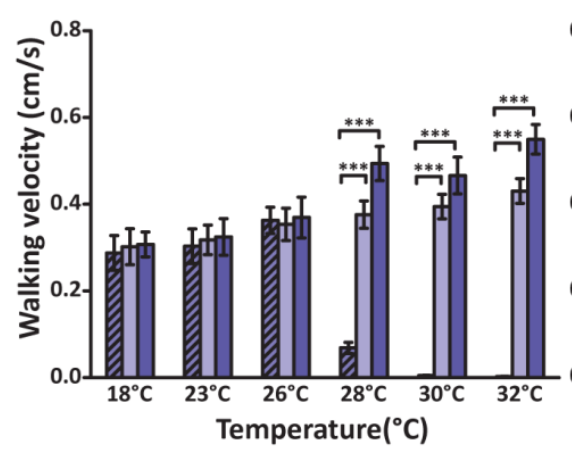

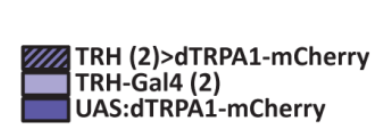

C
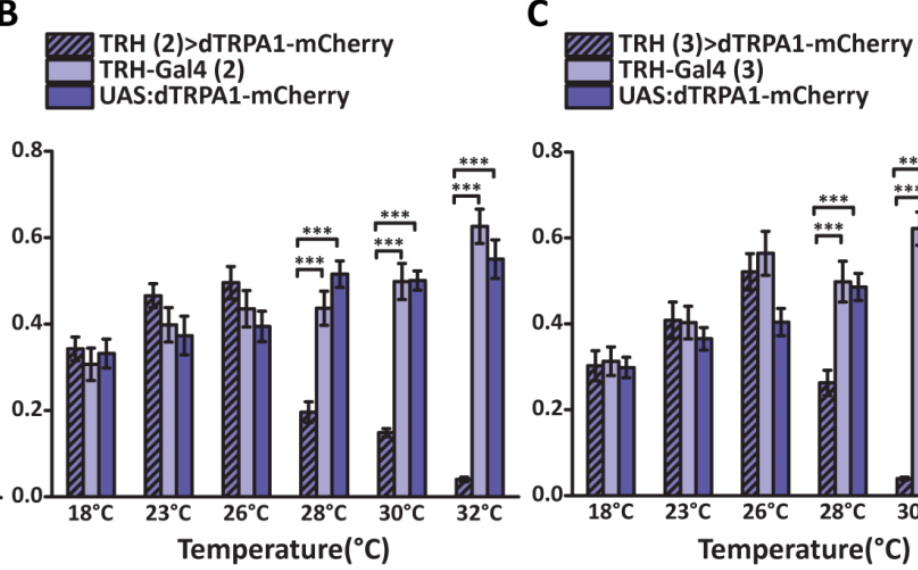

UAS:dTRPA1-mCherry

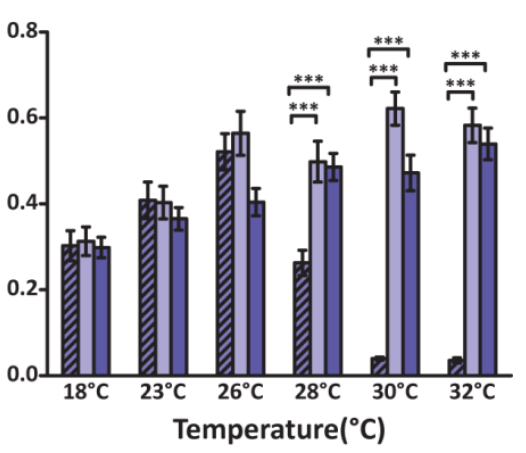

Figure 3.4. Effect of activation of serotoninergic neurons on the walking velocity. The flies expressing dTRPA1mCherry in serotoninergic neurons under the control of (A) TRH-Gal4 (1), (B) TRH-Gal4 (2) and (C) TRH-Gal4 (3) were tested in walking velocity paradigm. At the control temperatures $\left(18^{\circ} \mathrm{C}, 23^{\circ} \mathrm{C}, 26^{\circ} \mathrm{C}\right)$ no significant difference in the walking velocity between control and test groups was observed. Activating the serotoninergic neurons at $28^{\circ} \mathrm{C}, 30^{\circ} \mathrm{C}$ and $31^{\circ} \mathrm{C}$ caused a significant reduction in the walking velocity of the TRH>dTRPA1-mCherry flies in comparison to the heterozygous parental lines. One-Way ANOVA with post hoc Bonferroni correction. $(* * *)$ : P $\leq$ 0.001. $n=27$.

All three TRH-Gal4 lines showed the same trend in the decrement of the velocity (Figure 3.4). Based on the cell coverage and specificity of the TRH-Gal4 lines (Figure 3.3 M), the TRH-Gal4 (2) driver line (Sitaraman et al., 2012) was chosen for the expression of the transgenes in serotoninergic neurons in the following experiments.

\subsubsection{Activating serotoninergic neurons in the brain is sufficient for the reduction in the walking velocity}

The serotoninergic cells in the fly CNS are not restricted to the brain. The thoracic ganglion (TG) consists of four compartments: prothoracic, mesothoracic, metathoracic and abdominal neuromere. In the thoracic ganglion of the fly, $36 \pm 4$ (mean $\pm S D$ ) anti-serotonin immunoreactive cells were counted $(n=4)$. The majority of serotoninergic cells are located in the abdominal compartment of thoracic ganglion. The result of serotoninergic cell counting obtained in this 
study is comparable with a previous report by Valles and White (1988). All serotoninergic cells in the thoracic ganglion are covered by the used TRH-Gal4 driver line (Figure $3.5 \mathrm{~B}$ ).

To determine whether the serotoninergic cells in the thoracic ganglion contribute to the observed reduction in the velocity (section 3.3.1), the expression of dTRPA1-mCherry was suppressed in the thoracic ganglion using the teashirt-Gal80 line (Tsh-Gal80; Clyne et al., 2008) that suppresses Gal4-induced gene expression specifically in the thoracic ganglion. Flies carrying UAS:dTRPA1-mCherry on the $\mathrm{X}$ chromosome and Tsh-Gal80/cyo on the second chromosome were crossed with the TRH-Gal4 line. Using immunohistochemistry it was confirmed that in the offspring carrying UAS, Gal4 and Gal80 transgenes, the expression of the mCherry-dTRPA1 was repressed in the thoracic ganglion while expression in the brain region remained unaffected (Figure 3.5 A, B, C, D). The effect of activating serotoninergic neurons in the entire CNS was compared with the effect of an activation of serotonergic neurons in the brain only, using again the walking velocity paradigm. No significant difference in walking velocity between the different groups of animals was observed at $18^{\circ} \mathrm{C}$. At $32^{\circ} \mathrm{C}$ the activation of serotoninergic neurons in the entire CNS and the activation of serotonergic neurons in the brain only led to indistinguishable behavioral effects: both genotypes showed a significant reduction in walking velocity in comparison to the heterozygous parental lines. The result of this experiment confirmed the sufficiency of the serotoninergic neurons in the brain for the observed reduction in the walking velocity (Figure $3.5 \mathrm{E}$ ). 

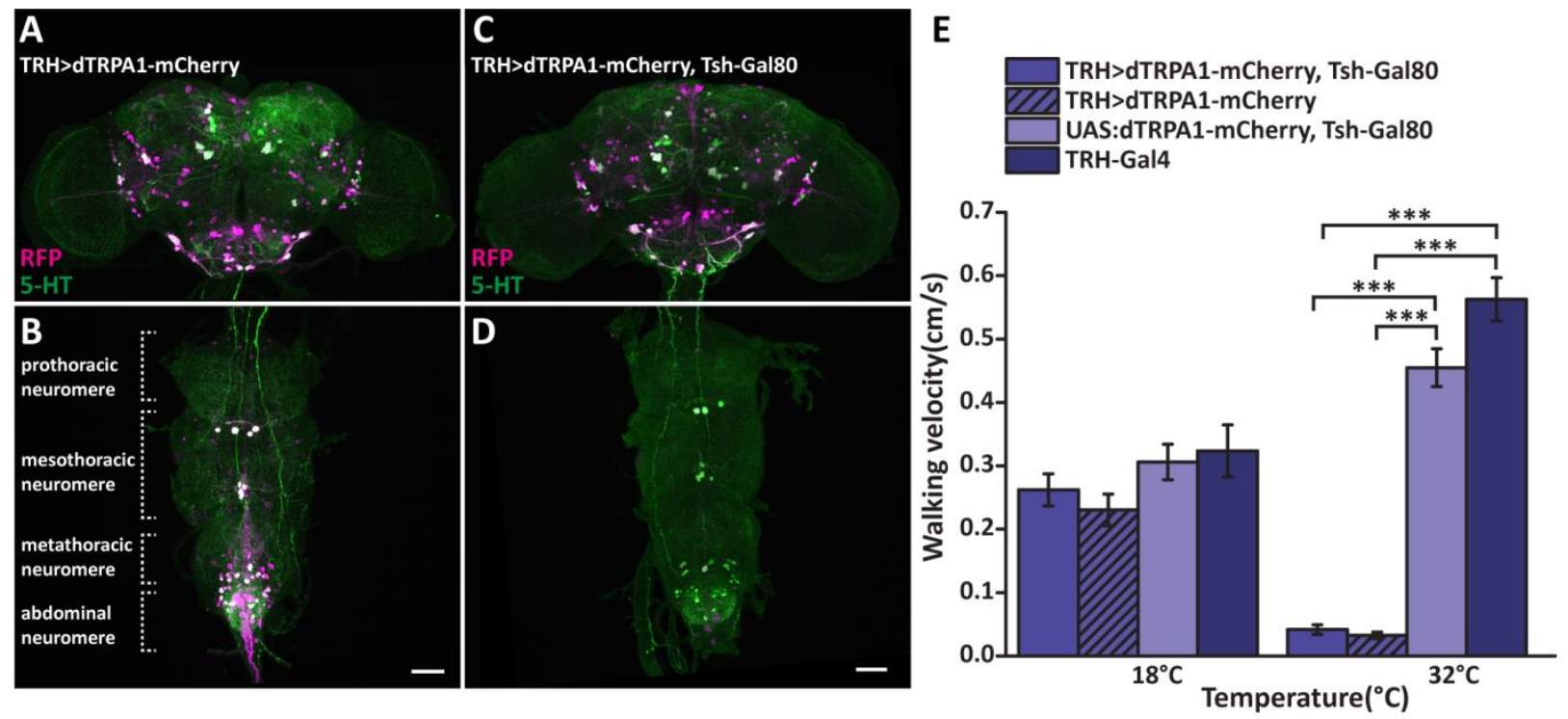

Figure 3.5. Repressing the expression of dTRPA1-mCherry in the thoracic ganglion. (A, B) The brain and thoracic ganglion of the fly expressing dTRPA1-mCherry under the control of the TRH-Gal4 driver line. The expression pattern of dTRPA1-mCherry was visualized using anti-RFP immunostaining (in magenta). The counterimmunostaining against serotonin (anti-5-HT) is shown in green. The white cells indicate the overlap of RFP and 5HT staining. Scale bar: $50 \mu \mathrm{m}$. (C, D) The brain and thoracic ganglion of the fly expressing dTRPA1-mCherry in the brain under the control of the TRH-Gal4 and Tsh-Gal80 lines. The double-staining against mCherry (anti RFP in magenta) and 5-HT (in green) shows that the expression of the dTRPA1-mCherry was suppressed in the thoracic

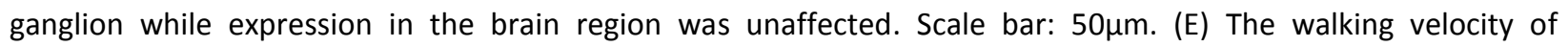
TRH>dTRPA1-mCherry flies (expressing dTRPA1-mCherry in the entire CNS) and TRH>dTRPA1-mCherry, Tsh-Gal80 flies (expressing dTRPA1-mCherry in the brain but not thoracic ganglion) and control groups (heterozygous parental lines) were compared at $18^{\circ} \mathrm{C}$ and $32^{\circ} \mathrm{C}$. In the experiment at $18^{\circ} \mathrm{C}$, no significant difference was observed between the groups. At $32^{\circ} \mathrm{C}$, both TRH $>$ dTRPA1-mCherry and TRH $>$ dTRPA1-mCherry, Tsh-Gal80 groups showed a significant reduction in walking velocity in comparison to the parental lines. Furthermore, no significant difference between walking velocity of the TRH>dTRPA1-mCherry and TRH>dTRPA1-mCherry, Tsh-Gal80 groups was observed. One-Way ANOVA with post hoc Bonferroni correction. $\left({ }^{* * *}\right): \mathrm{P} \leq 0.001 . \mathrm{n}=27$.

\subsubsection{The effect of thermogenetic activation of serotoninergic neurons on the animals' responsiveness to external mechanical stimuli}

The result of the walking velocity experiment raised the question whether the observed reduction in the velocity was merely a motor deficit or indeed due to the regulatory effect of serotonin on the arousal level. Therefore, the effect of the activation of serotoninergic neurons on the flies' walking ability and their responsiveness to external stimuli was tested in a negative geotaxis, a shock avoidance and a flight assay paradigm and directly compared to the effect 
that is obtained when motor neurons are activated. In general, the artificial activation of motor neurons through the dTRPA1 channel causes paralysis of the fly. The dTRPA1-mCherry was expressed in motor neurons using the D42-Gal4 driver line (Parkes et al., 1998; Figure 3.6). For the following experiments, the flies expressing dTRPA1-mCherry under the control of TRH-Gal4 or D42-Gal4 were used as the test groups (TRH test group and D42 test group) and the heterozygous parental lines were used as control groups.

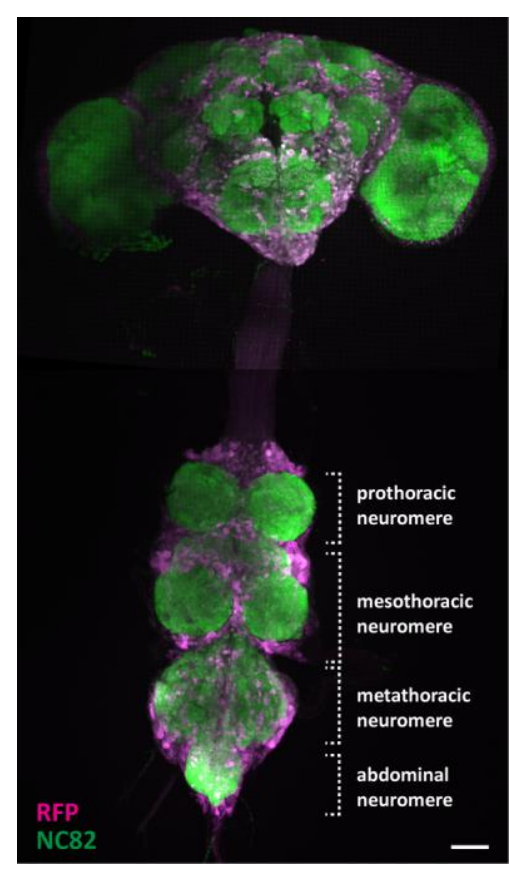

Figure 3.6. The Expression pattern of D42-Gal4 using UAS:dTRPA1-mcherry. The expression pattern of dTRPA1-mcherry under the control of D42 driver line was visualized by immunostaining using anti-RFP against mCherry (magenta) and anti-bruchpilot (NC82) (green) in the brain and the thoracic ganglion. Scale bar: $50 \mu \mathrm{m}$.

\subsubsection{Negative geotaxis}

Flies typically show negative geotaxis: they climb against gravity and towards a light source when they are agitated (Benzer, 1976). Therefore, both the movement ability of the flies and also their responsiveness to mechanical stimulation can be quantified in a geotaxis assay. In a simple geotaxis assay (assay 1; section 2.2.5.3), both TRH and D42 flies (test and control groups) showed a high score for negative geotaxis at the control temperature $\left(18^{\circ} \mathrm{C}\right)$. At $32^{\circ} \mathrm{C}$, the $\mathrm{D} 42$ test flies paralyzed; hence they did not climb in the tube. Also the test group, in which serotonergic neurons were activated by the temperature, showed a significant reduction in the geotaxis score in comparison to the controls (Figure 3.7 A, B). But in contrast to flies with activated motor neurons, flies whose serotonergic neurons were activated did not paralyze: they were able to move, as indicated by scores above 0 . To quantify that more accurately, flies 
were next tested with the Benzer geotaxis paradigm (modified geotaxis assay with six tubes, assay 2; section 2.2.5.3). In this assay, flies were distributed in six tubes and they had to climb up five times, i.e., repeatedly, to achieve a score. Therefore, the differentiation between nonclimbing flies and flies with reduced climbing ability was possible. At $18^{\circ} \mathrm{C}$, all the flies performed similarly in test and control groups. But at $32^{\circ} \mathrm{C}$, for both $\mathrm{TRH}$ and D42 experiments, the geotaxis scores decreased significantly in the test groups in comparison to the control groups (Figure 3.7 C, D). Again, flies with activated motor neurons were not able to move at all, no score above 0 was achieved. Flies with activated serotonergic neurons did move, although less when compared to controls, and therefore achieved a geotaxis score above 0 . This is reflected by a significant difference between geotaxis indices of these two groups (negative geotaxis index \pm SEM: $0 \pm 0$ for $D 42$ test flies and $0.3 \pm 0.05$ for TRH test flies). This result was consistent with the result obtained from the geotaxis assay 1. 
A

\begin{tabular}{|c|}
\hline TRH>dTRPA1-mCherry \\
\hline TRH-Gal4 \\
\hline UAS:dTRPA1-mCherry \\
\hline
\end{tabular}

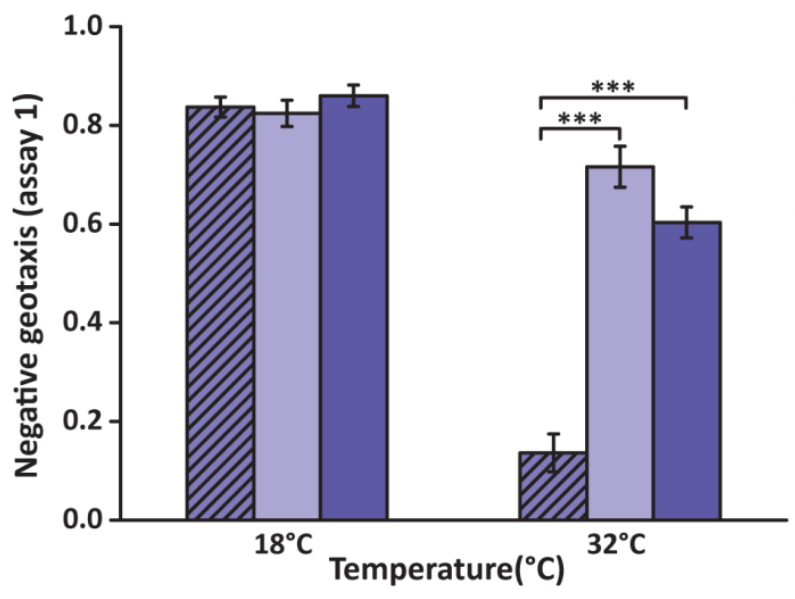

C

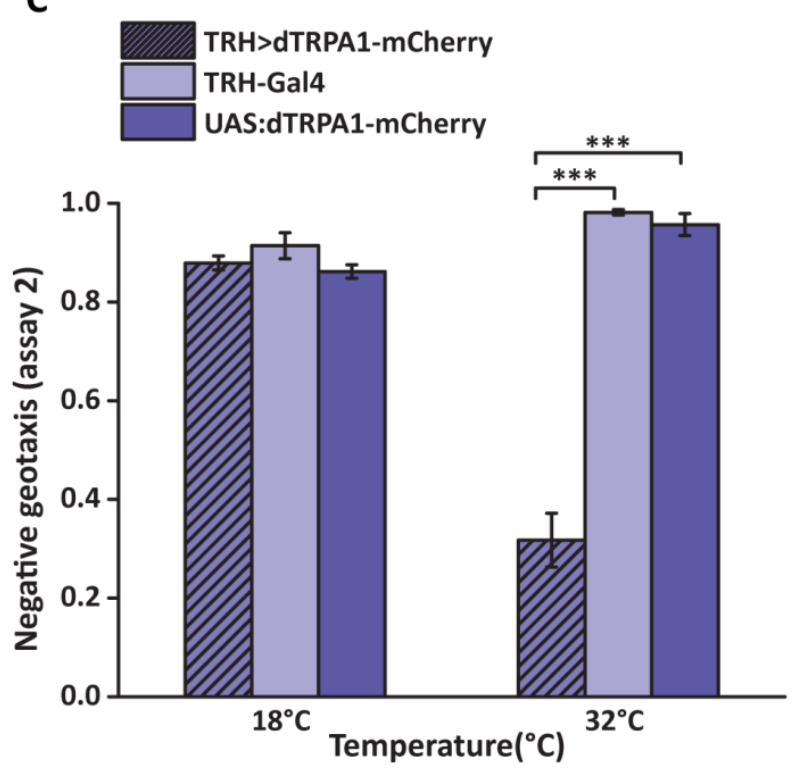

B
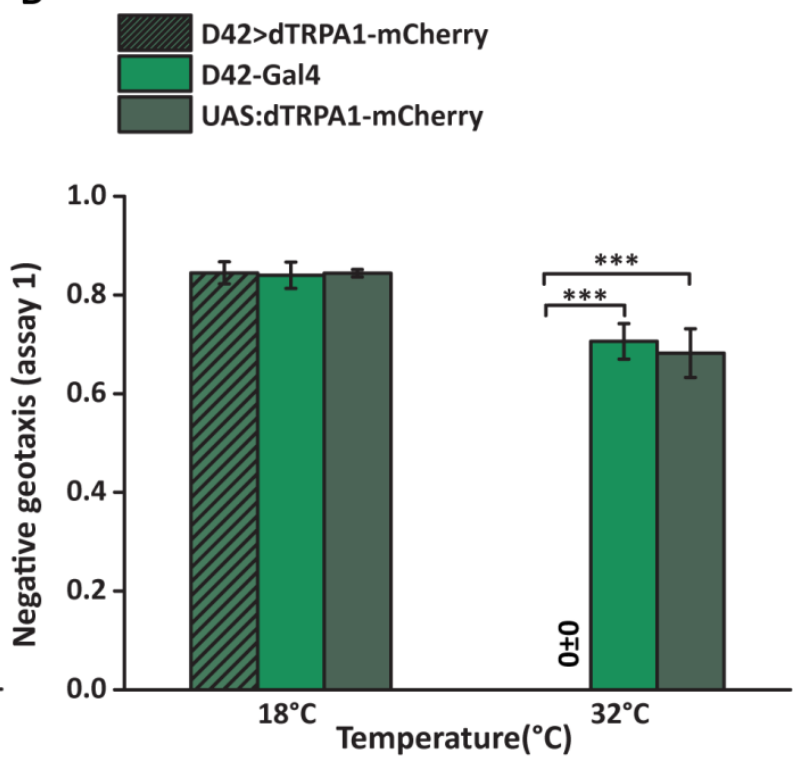

D
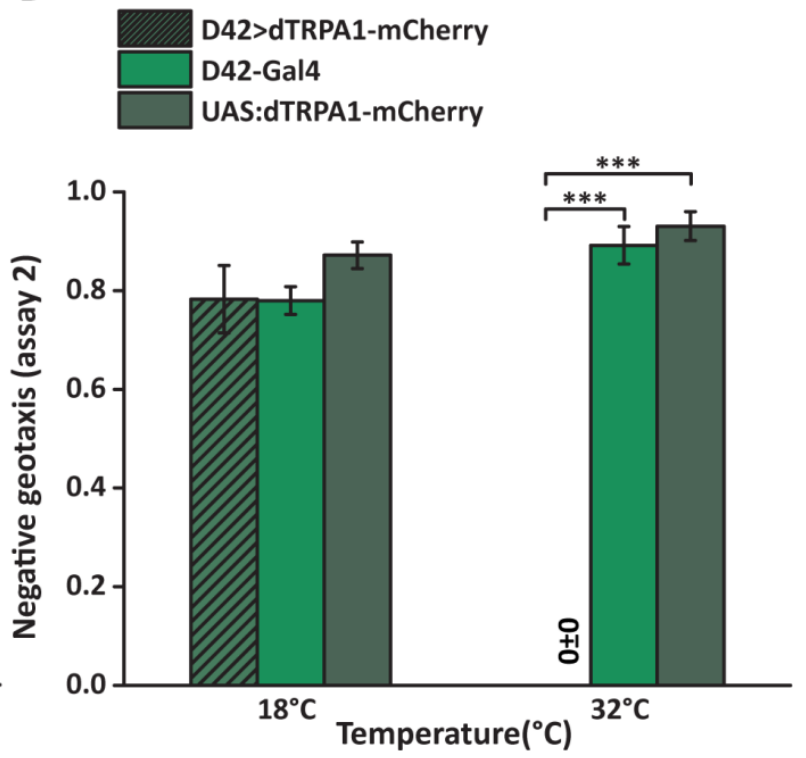

Figure 3.7. Negative geotaxis assay. (A) The TRH>dTRPA1-Cherry flies and the heterozygous parental lines were tested in the negative geotaxis assay 1 at $18^{\circ} \mathrm{C}$ and $32^{\circ} \mathrm{C}$. No difference in the Negative geotaxis scores was observed at $18^{\circ} \mathrm{C}$. However, the activation of serotoninergic neurons at $32^{\circ} \mathrm{C}$ caused significant reduction in the climbing ability of the TRH $>$ dTRPA1-mCherry flies in comparison to the control groups. (B) The D42>dTRPA1-Cherry flies and the heterozygous parental lines were tested in the negative geotaxis assay 1 at $18^{\circ} \mathrm{C}$ and $32^{\circ} \mathrm{C}$. No difference in the negative geotaxis scores was observed at $18^{\circ} \mathrm{C}$. But activation of motor neurons $\left(\right.$ at $32^{\circ} \mathrm{C}$ ) caused complete paralysis of D42>dTRPA1-Cherry flies. Therefore, the negative geotaxis score for these flies was $0 \pm 0$ (mean \pm SEM). (C) The TRH>dTRPA1-Cherry flies and the heterozygous parental lines were tested in the negative geotaxis assay 2 at $18^{\circ} \mathrm{C}$ and $32^{\circ} \mathrm{C}$. No difference in the negative geotaxis scores was observed at $18^{\circ} \mathrm{C}$. However, the activation of serotoninergic neurons at $32^{\circ} \mathrm{C}$ caused a significant reduction in the negative geotaxis score of the 
TRH>dTRPA1-mCherry flies in comparison to the control groups. (D) The D42>dTRPA1-Cherry flies and the heterozygous parental lines were tested in the negative geotaxis assay 2 at $18^{\circ} \mathrm{C}$ and $32^{\circ} \mathrm{C}$. No difference in the negative geotaxis scores was observed at $18^{\circ} \mathrm{C}$. However, the negative geotaxis score for D42>dTRPA1-Cherry flies was $0 \pm 0($ mean $\pm S E M)$ at $32^{\circ} \mathrm{C}$. One-Way ANOVA with post hoc Bonferroni correction. $\left({ }^{* *}\right): P \leq 0.001$. $n=5$.

In summary, the reduction in the movement caused by activation of the serotoninergic neurons is distinguishable from a locomotion deficit induced by manipulation of motor neurons.

\subsubsection{The activation of serotonergic neurons does not impair electric shock avoidance}

Since arousal is also defined by the behavioral responsiveness to external stimuli, the next question was whether the activation of serotonergic neurons would affect the responsiveness towards a life-threatening, aversive stimulus. In the shock avoidance paradigm (section 2.2.5.4), the flies were transferred to the shock tube in the T-maze apparatus. The flies had 1 minute to escape from the shock tube to the safe tube, which requires, of course, that the animals are able to move. By counting flies on each site the shock avoidance index was calculated. Again, the performance of flies with an activated serotonergic system (TRH) was compared to the performance of flies that paralyze due to motor neuron activation (D42). In both D42 and TRH experiments at $22^{\circ} \mathrm{C}$, test and control groups did not show any significant difference in shock avoidance index. But at $32^{\circ} \mathrm{C}$, the flies expressing dTRPA1-mCherry in the motor neurons were paralyzed. Therefore, these flies were not able to avoid the electric shock. However, in TRH experiments, the avoidance index of the test group was not significantly different from the genetic controls (Figure 3.8). Based on the result of this experiment, it can be concluded that the observed effect of activation of serotoninergic neurons on reduction in walking velocity was not a motor deficit phenotype, and the flies were able to show a normal escape response in the presence of electric shock. It can be further concluded, that the activation of serotoninergic neurons does not affect the behavioral responsiveness of the animal to a very strong aversive stimulus, i.e. to an electric shock stimulus. 
A
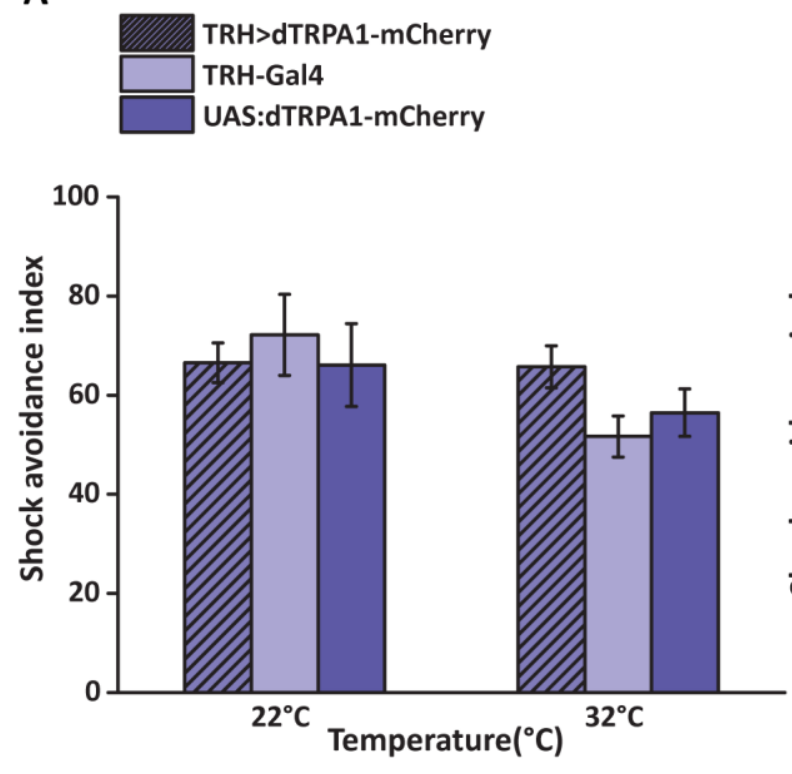

B
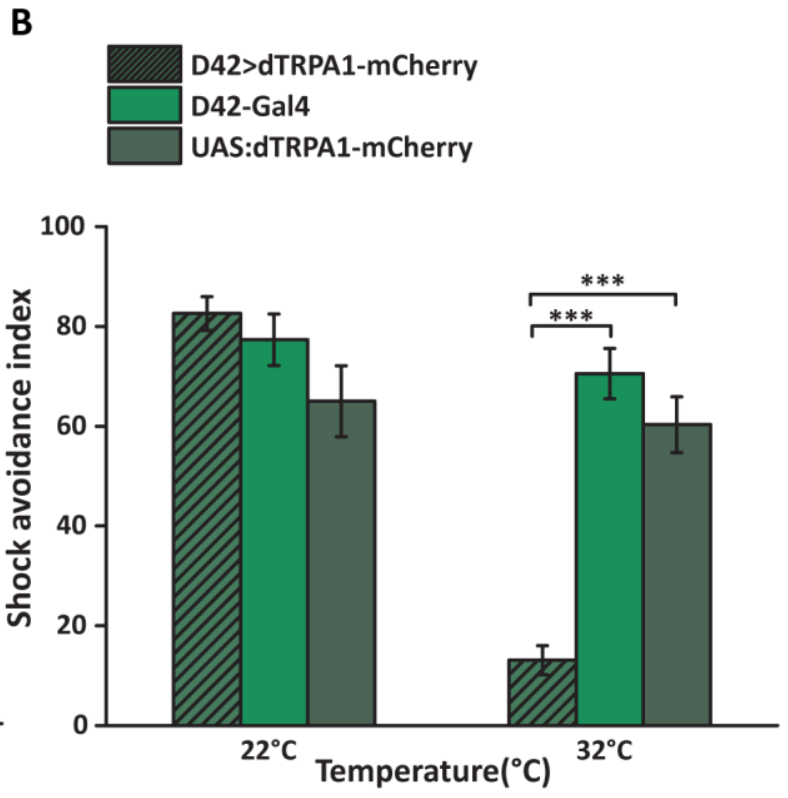

Figure 3.8. Shock avoidance experiment. (A) The flies expressing dTRPA1-mCherry under the control of TRH-Gal4 driver line were tested in shock avoidance experiment at $22^{\circ} \mathrm{C}$ and $32^{\circ} \mathrm{C}$. The TRH $>d T R P A 1-m C h e r r y$ flies and the control groups (heterozygous parental lines) did not show significant difference in shock avoidance neither at $22^{\circ} \mathrm{C}$ nor at $32^{\circ} \mathrm{C}$. (B) The D42>dTRPA1-mCherry flies were compared to the heterozygous parental lines in shock avoidance paradigm at $22^{\circ} \mathrm{C}$ and $32^{\circ} \mathrm{C}$. At the control temperature $\left(22^{\circ} \mathrm{C}\right)$ no significant difference was observed between the groups. However, at $32^{\circ} \mathrm{C}$, activation of motor neurons caused complete paralysis in D42>dTRPA1mCherry flies. Thereby, a significantly low shock avoidance index in comparison to the controls was observed. OneWay ANOVA with post hoc Bonferroni correction. $\left(^{* * *}\right): P \leq 0.001 . n=10-11$.

\subsubsection{The activation of serotonergic neurons does not impair forced flight in a flight assay}

The third test for determining the movement ability and responsiveness of the flies to a disturbing external stimulus was the flight assay (section 2.2.5.5). Flies were relieved first into an oil coated container. To avoid falling to the ground, the fly had to fly and got trapped in the oil coating the container. Thereby, the responsiveness could be determined by counting the flies at different areas of the wall (or at the ground). Again, the performance of flies with an activated serotonergic system (TRH) was compared to the performance of flies that paralyze due to motor neuron activation (D42). In both TRH and D42 experiments the test and their genetic control groups showed no significant difference in the flight index at $22^{\circ} \mathrm{C}$. However, at $32^{\circ} \mathrm{C}$ the activation of dTRPA1 channel led to paralysis of the D42>dTRPA1-mCherry files. Therefore, these flies showed a significantly decreased flight index in comparison to the 
controls. In TRH experiment, the TRH>dTRPA1-mCherry and heterozygous parental lines did not show any significant difference in the flight index (Figure 3.9). This experiment confirmed that flies with activated serotoninergic neurons respond with normal activity when they are forced to fly.

A
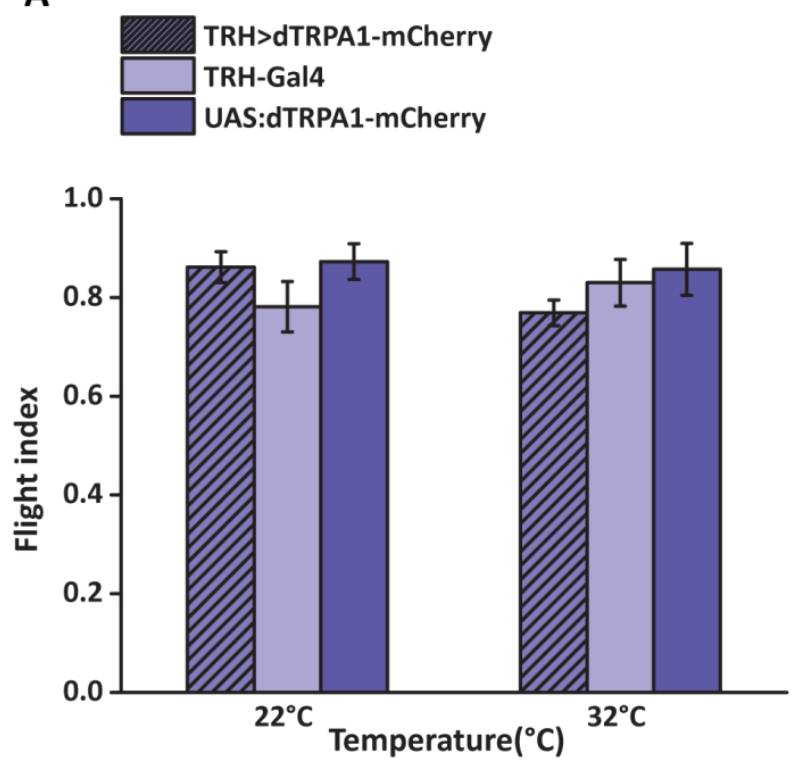

B
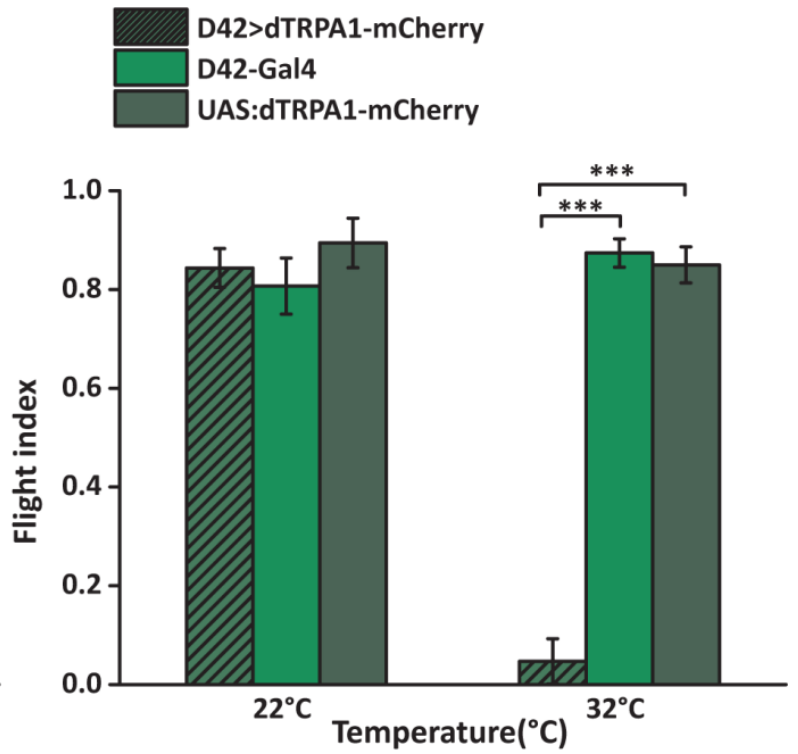

Figure 3.9. Flight assay. (A) The flies expressing dTRPA1-mCherry under control of a TRH-Gal4 driver line were tested in a flight assay at $22^{\circ} \mathrm{C}$ and $32^{\circ} \mathrm{C}$. The TRH $>$ dTRPA1-mCherry flies and the control groups (heterozygous parental lines) did not any show significant difference in flight index neither at $22^{\circ} \mathrm{C}$ nor at $32^{\circ} \mathrm{C}$. (B) The D42>dTRPA1-mCherry flies were compared to the heterozygous parental lines in the flight assay at $22^{\circ} \mathrm{C}$ and $32^{\circ} \mathrm{C}$. At the control temperature $\left(22^{\circ} \mathrm{C}\right)$ no significant difference was observed between the groups. However, at $32^{\circ} \mathrm{C}$, activation of motor neuron caused complete paralysis in D42>dTRPA1-mCherry. Thereby, a significantly low flight index in comparison to the controls was observed. One-Way ANOVA with post hoc Bonferroni correction. $\left({ }^{* * *}\right)$ : P $\leq$ 0.001. $n=5$.

Based on the experiments described above (negative geotaxis, shock avoidance and flight assay), activation of serotoninergic neurons causes a general reduction in activity on the flies which is correlated with decrement in the walking velocity. However, the responsiveness of the flies to strongly aversive external stimuli was not completely impaired by activation of serotoninergic neurons and in the presence of agitative and mechanical stimuli, the optimal arousal level could be restored. 


\subsubsection{Thermogenetic activation of serotoninergic neurons prevents starved flies from eating}

The next question was whether in the presence of internal stimulus like hunger, the arousal level in the flies could be restored in the presence of food, i.e., external appetitive olfactory, visual and gustatory stimuli, while the serotoninergic neurons were artificially activated. For answering this question, the feeding behavior of starved flies expressing dTRPA1-mCherry under the control of the TRH-Gal4 driver line (as the test group) and the starved heterozygous parental lines (as the genetic controls) was compared. To do so, starved flies were fed for 45 min on food containing red dye, subsequently decapitated and homogenized, and food uptake was determined by the absorbance of this homogenate at $500 \mathrm{~nm}$ (section 2.2.5.6). At $18^{\circ} \mathrm{C}$ the food intake of the test and control flies was not found to be significantly different. However, at $32^{\circ} \mathrm{C}$, due to the activation of serotoninergic neurons a drastic reduction in the amount of food uptake in TRH<dTRPA1-mCherry flies in comparison to the control groups was observed (Figure 3.10 A).

A

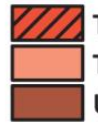

TRH >dTRPA1-mCherry

TRH-Gal4

UAS:dTRPA1-mCherry

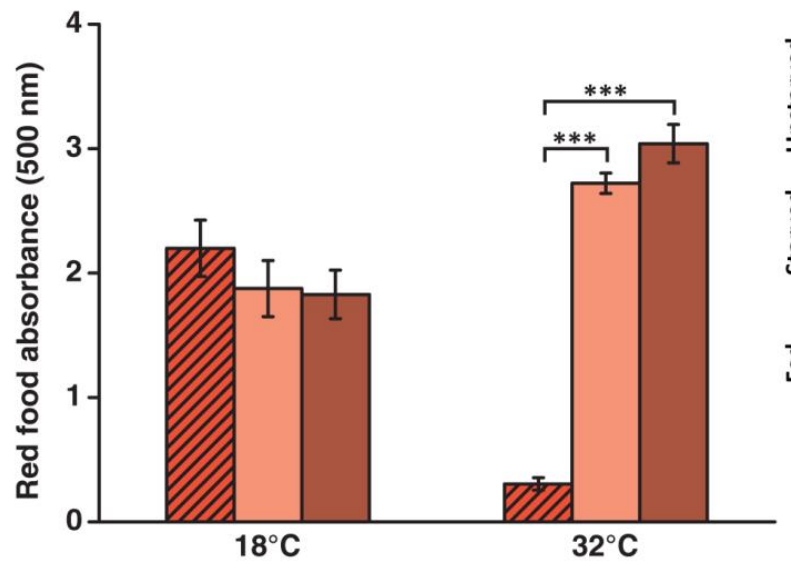

B

TRH>dTRPA1-mCherry TRH-Gal4 UAS:dTRPA1-mCherry

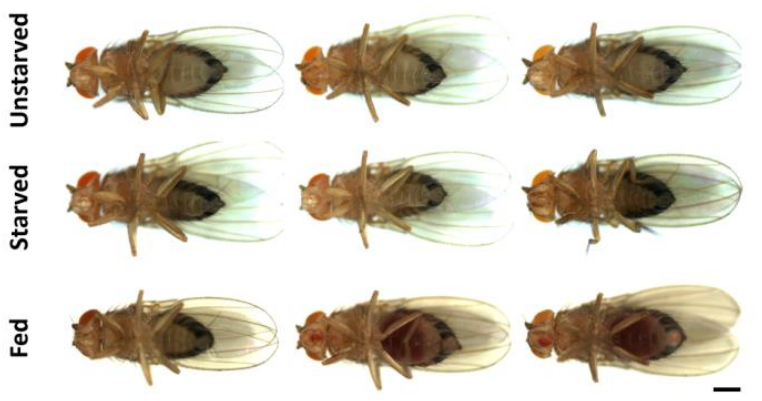

Figure 3.10. Feeding assay. (A)The starved TRH<dTRPA1-mCherry flies and the heterozygous parental lines were tested for the amount of red food intake at $18^{\circ} \mathrm{C}$ and $32^{\circ} \mathrm{C}$. At the control temperature $\left(18^{\circ} \mathrm{C}\right)$, no significant difference in food intake between test and controls was observed. However, at $32^{\circ} \mathrm{C}$, while the serotoninergic neurons were activated, the red food absorbance of the TRH<dTRPA1-mCherry flies got significantly reduced in comparison to the heterozygous parental lines. One-Way ANOVA with post hoc Bonferroni correction. $(* * *)$ : P $\leq$ 0.001. $n=5$. (B) The example pictures of TRH>dTRPA1-mcherry and the heterozygous parental flies during three 
phases (before starvation, after starvation and then after transferring to the red food at $32^{\circ}$ ) are shown. Scale bar: $500 \mu \mathrm{m}$.

The TRH>dTRPA1-mCherry flies and heterozygous parental lines were in the same health state before starvation. Due to the starvation, all three groups lost weight in comparable amounts. This could be easily observed by visual inspection of the fly's appearance. After transferring flies to the red food vials at $32^{\circ} \mathrm{C}$, parental controls got the lost weight back. However, the TRH $<$ dTRPA1-mCherry flies did not regain the lost weight (Figure 3.10 B). These results show that the (regulatory) effect of the serotoninergic neurons on the arousal state in the flies modulates the responses to the internal stimulus like hunger. Or it modulates the responsiveness towards appetitive stimuli. This experiment does not distinguish between these two possibilities. Are they less hungry? Or are they equally hungry and just not motivated to start an action, like feeding? This question could not be answered in the course of this thesis and remains to be investigated.

\subsection{Thermogenetic blocking transmission from serotoninergic neurons increases walking velocity}

When the activation of the serotoninergic neurons leads to reduced arousal level (section 3.3), what is the consequence of blocking of these neurons? For silencing the synaptic transmission in the serotoninergic neurons, temperature sensitive shibire $\left(\mathrm{Shi}^{(\mathrm{ts})}\right)$ was expressed under the control of TRH-Gal4. For testing for the animals' arousal level, the flies expressing Shi ${ }^{(t s)}$ in the serotoninergic neurons as the test group and heterozygous parental lines as the control groups were tested in the walking velocity paradigm described above. At permissive temperatures $\left(18^{\circ} \mathrm{C}\right.$ and $\left.25^{\circ} \mathrm{C}\right)$, when the signal transduction in serotoninergic neurons was intact, the walking velocity of the test and control groups was not significantly different. However, in the experiment at restrictive temperature $\left(32^{\circ} \mathrm{C}\right)$, the signal transduction in serotoninergic neurons was inhibited and the walking velocity of the TRH $>S h i^{(t s)}$ flies was significantly higher than the heterozygous parental lines. Based on this result, the regulatory effect of the serotoninergic neurons on arousal state was found to be bidirectional, in a way that activation of these cells decreases the arousal level while blocking them causes a higher arousal level in the flies (Figure 3.11). 


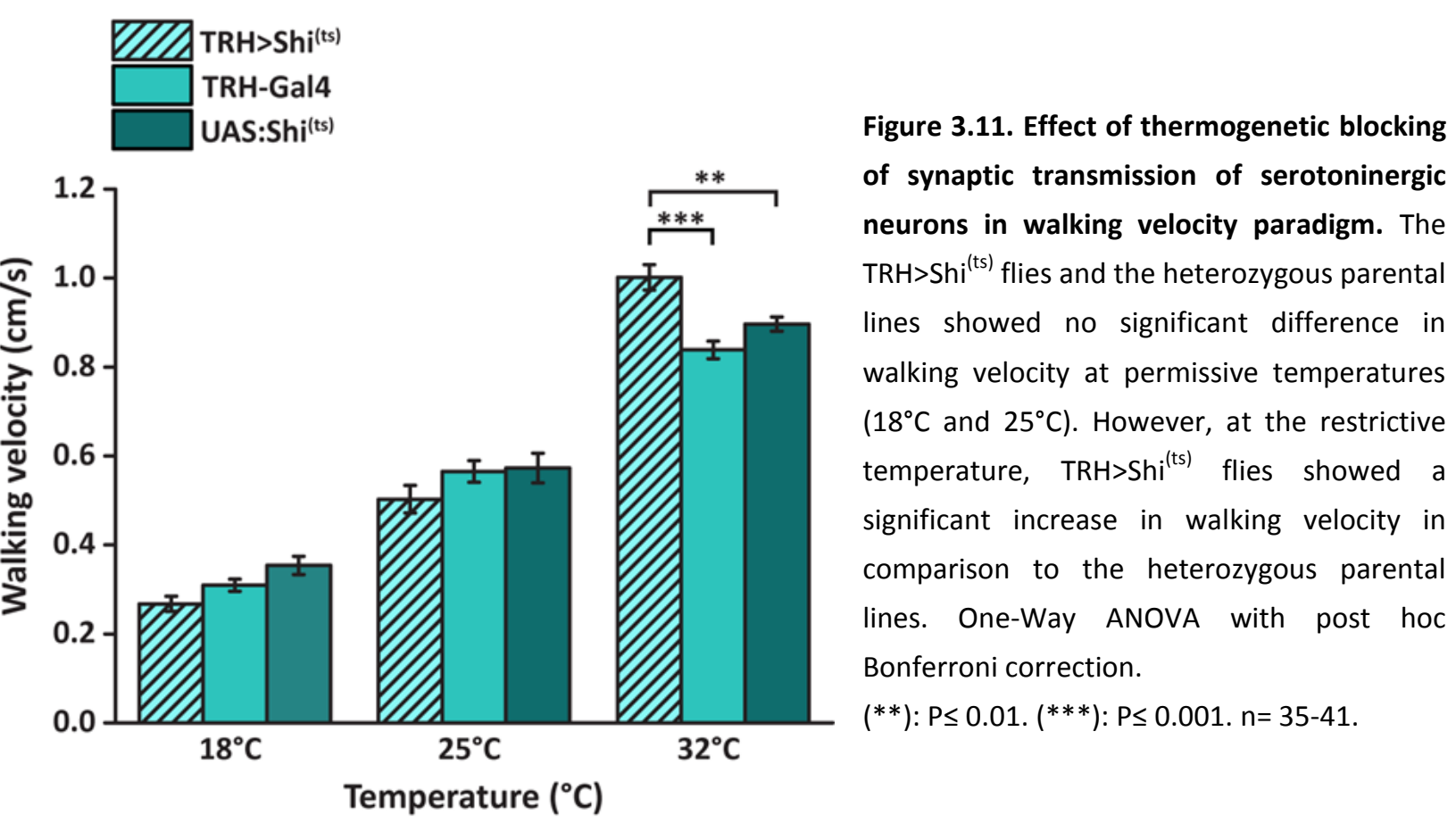

\subsection{Regulatory effect of serotoninergic neurons on the endogenous arousal state}

Since the results from the experiments above implicate an involvement of serotoninergic neurons in the regulation of a general arousal level in flies, the modulatory effect of serotoninergic neurons on activity and sleep as endogenous arousal states was investigated in the following experiment. For this purpose, the movement of the flies was monitored over time using a "Drosophila Activity Monitoring" (DAM) system. In order to investigate the property of the dTRPA1 channel activity over time, dTRPA1-mCherry was expressed in motor neurons using the D42 driver line. The activation of motor neurons leads to a paralysis of the flies. To determine the functionality of dTRPA1-mCherry during continuous activation, the walking velocity of the D42>dTRPA1-mCherry flies and the heterozygous parental lines was monitored in the single fly assay of walking velocity paradigm for $3 \mathrm{~h}$ at $18^{\circ} \mathrm{C}$ and $32^{\circ} \mathrm{C}$. The experiment at $18^{\circ} \mathrm{C}$ showed that in the first 30 minutes the activity of the flies got reduced in a comparable way in both D42>dTRPA1-mCherry and heterozygous parental lines. At $32^{\circ} \mathrm{C}$, the heterozygous parental lines showed a comparable trend to enhance their walking velocity (which has been also observed in 3.3.1). In the D42>dTRPA1-mCherry flies, the activity of the dTRPA1 channel in 
the motor neurons led to paralysis of the flies in the first one hour, however they started to recover from paralysis after about $70 \mathrm{~min}$. The walking velocity of these flies increased over time. Based on the result of this experiment it was concluded that dTRPA1-mCherry is not the appropriate tool for activating neurons for a long duration of time (Figure 3.12).

A

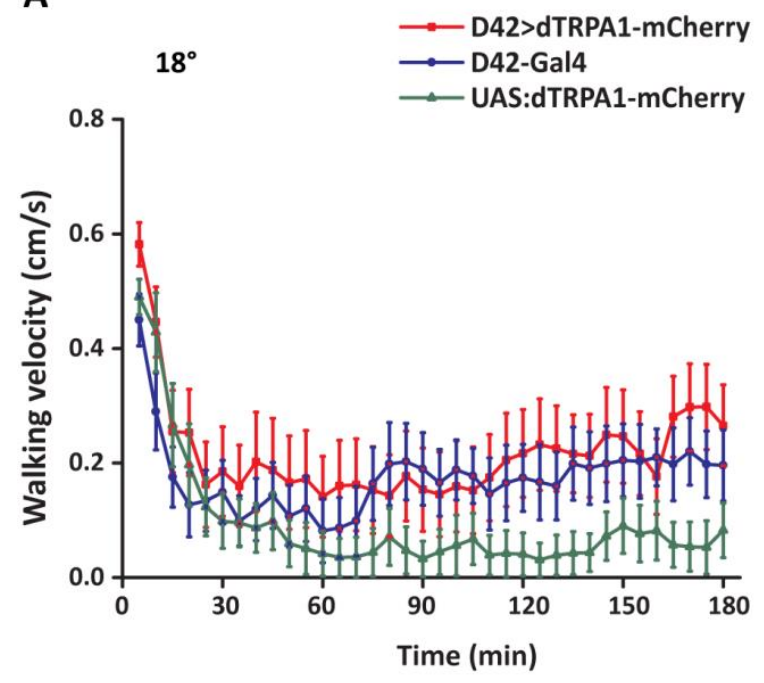

B

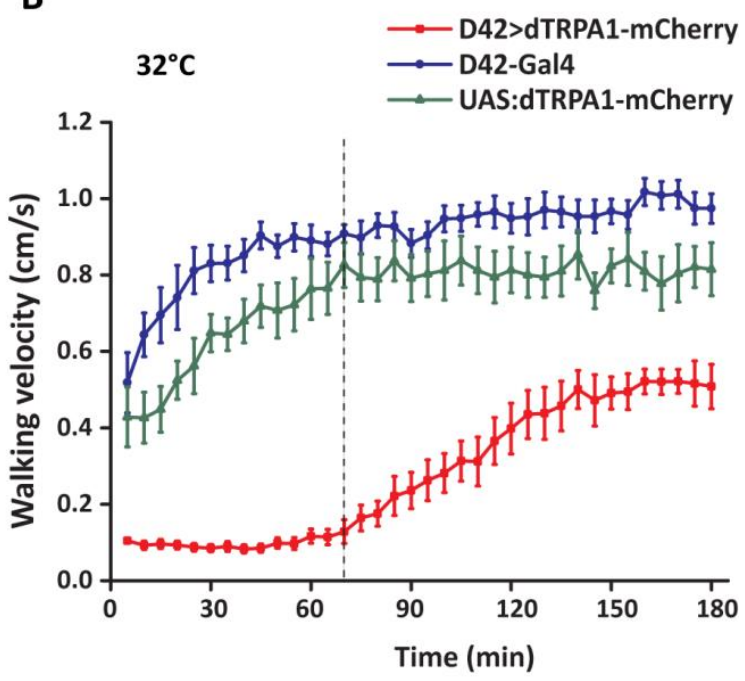

Figure 3.12. Characterizing the dTRPA1-mCherry functionality over long-term, continuous experiments. The walking velocity of the D42>dTRPA1-mCherry flies and the heterozygous parental lines were monitored in a single fly assay of walking velocity paradigm for $3 \mathrm{~h}$ at $18^{\circ} \mathrm{C}$ and $32^{\circ} \mathrm{C}$. At $18^{\circ} \mathrm{C}$, walking velocity of all the groups over the $3 \mathrm{~h}$ time showed a comparable trend. At $32^{\circ} \mathrm{C}$, D42>dTRPA1-mCherry flies got paralyzed in the first hour due to the activation of their motor neurons. After about $70 \mathrm{~min}$, the D42>dTRPA1-mCherry flies started to recover from paralysis and the walking velocity of these flies increased over time. In the graphs each dot indicates the mean velocity \pm SEM for each 5 min time bin. $n=9$.

Therefore, in order to investigate the modulatory effect of serotoninergic neurons on activity and sleep, the signal transduction in serotoninergic neurons was blocked using Shi ${ }^{(t)}$ and the activity of the flies was monitored for $72 \mathrm{~h}$ in the $12 / 12 \mathrm{~h}$ light-dark condition in the DAM system (section 2.2.5.7). The flies expressing $\mathrm{Shi}^{(\mathrm{ts})}$ in control of TRH-Gal4 as the test group were compared with the heterozygous parental lines as the control groups. In the experiment at $22^{\circ} \mathrm{C}$, the test and the control groups performed without any significant difference in the amount of activity and sleeping time (Figure 3.13 A, C, E, G). The data from the experiment at $32^{\circ} \mathrm{C}$ showed that the day activity and sleep amount of the test and control flies was not significantly different from each other. However, in the dark phase, the TRH $>\mathrm{Shi}^{\left({ }^{(t)}\right)}$ flies showed 
significantly increased activity and decreased sleep amount in comparison to the heterozygous parental lines (Figure $3.13 \mathrm{~B}, \mathrm{D}, \mathrm{F}, \mathrm{H}$ ).
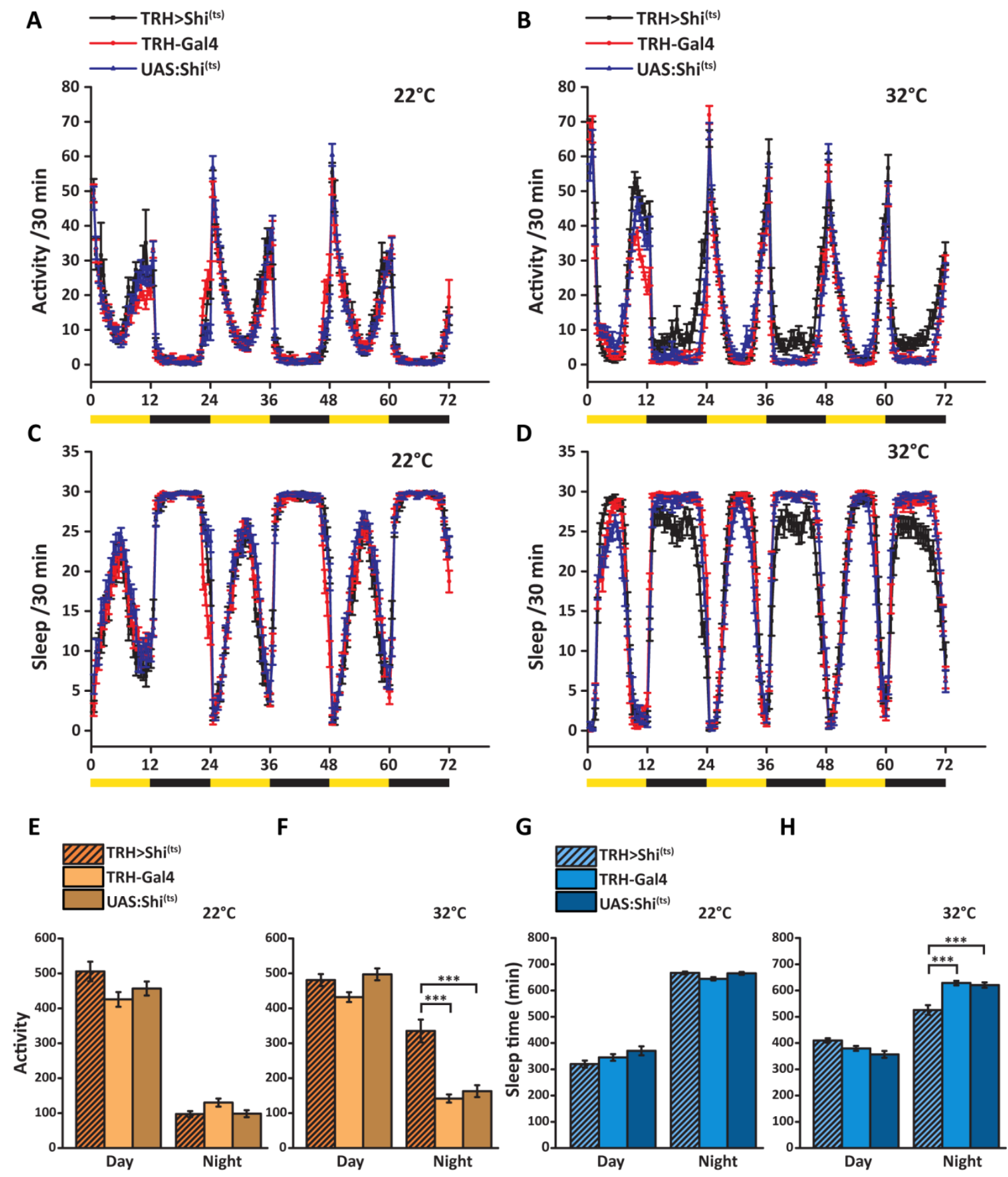

Figure 3.13. Effect of blocking synaptic output from serotoninergic neurons on activity and sleep. (A, B) The $\mathrm{TRH}>\mathrm{Shi}^{(\mathrm{ts})}$ flies and heterozygous parental lines were monitored in the DAM system for $72 \mathrm{~h}$ in the $12 / 12 \mathrm{~h}$ light- 
dark cycles at $22^{\circ} \mathrm{C}$ and $32^{\circ} \mathrm{C}$. The activity/30min shows the number of beam crossings per 30 min during $72 \mathrm{~h}$ (mean $\pm S E M)$. The yellow-black bars below the graphs indicate the light and dark cycles. (C, D) The TRH $>$ Shi $^{\left({ }^{(t)}\right)}$ flies and heterozygous parental lines were monitored in the DAM system for $72 \mathrm{~h}$ in the $12 / 12 \mathrm{~h}$ light-dark cycles at $22^{\circ} \mathrm{C}$ and $32^{\circ} \mathrm{C}$. The sleep $/ 30 \mathrm{~min}$ shows the time flies spent on sleeping (no movement for at least 5 minutes) per 30 min during $72 \mathrm{~h}$ (mean $\pm S E M)$. The yellow-black bars below the graphs show the light and dark cycles. $(E, F)$ The total day and night activity of the TRH>Shi ${ }^{(t s)}$ flies and heterozygous parental lines in $72 \mathrm{~h}$. At the control temperature $\left(22^{\circ} \mathrm{C}\right)$ no difference in the amount of activity (day and night) between TRH $>$ Shi ${ }^{(t)}$ flies and the control flies was observed. The amount of daily activity of the flies with blocked serotoninergic neurons (TRH>Shi ${ }^{(t s)}$ ) and the control flies was not significantly different. However, these flies showed elevated night activity in comparison to the controls at restrictive temperature $\left(32^{\circ} \mathrm{C}\right)$. $(\mathrm{G}, \mathrm{H})$ The total sleep time $(\mathrm{min})$ of the $\mathrm{TRH}>\mathrm{Shi}{ }^{(\mathrm{ts})}$ flies and the heterozygous parental lines in $72 \mathrm{~h}$. At the permissive temperature, no difference between total sleep (day and night) was observed between the groups. At the restrictive temperature, blocking the serotoninergic neurons did not affect the daily sleep. However, the night sleep was significantly less in $\mathrm{TRH}>\mathrm{Shi}^{(\mathrm{ts})}$ flies in comparison to the heterozygous parental lines. One-Way ANOVA with post hoc Bonferroni correction. $\left.{ }^{* * *}\right): P \leq 0.001 . n=42$.

In conclusion, the flies with the blocked signal transduction in serotoninergic neurons slept less than controls in the night, although the amount of sleep during day time was not significantly different from the controls. Based on this result it can be concluded, that the modulatory effect of serotoninergic neurons on the arousal state affects the activity and sleep amount in the night, but not the period of the entrained circadian rhythm.

\subsection{Is it the neurotransmitter serotonin that is involved in the regulation of arousal state?}

Based on the result of experiments above, the serotoninergic neurons were found to be involved in modulating the arousal state in flies. In order to investigate whether it is indeed the neurotransmitter serotonin released by these neurons that is causing this modulation (or any other co-transmitter), serotonin synthesis was pharmacologically reduced and the walking velocity of the flies was measured. The drug para-chlorophenylalanine (PCPA) inhibits the biosynthesis of serotonin by blocking the tryptophan hydroxylase (Koe and Weissmann, 1966). This drug was fed to the flies with different dosages and for various durations. The effect of PCPA was verified by quantification and comparison of anti 5-HT staining in the drug-fed and the untreated CS flies. Feeding the flies for four days at $25^{\circ} \mathrm{C}$ with the food containing PCPA showed the strongest reduction in the immunohistochemically detectable serotonin level of flies (Figure 3.14 A, B, C). The starved TRH>dTRPA1-mCherry flies and the heterozygous 
parental lines were fed with red food containing PCPA and the control red food (no PCPA) for four days at $25^{\circ} \mathrm{C}$. The walking velocity of these flies was measured at $18^{\circ} \mathrm{C}$ and $32^{\circ} \mathrm{C}$. The flies with PCPA treatment and no treatment did not show any significant difference at $18^{\circ} \mathrm{C}$ (Figure 3.14 D). At $32^{\circ} \mathrm{C}$, the TRH $>\mathrm{dTRPA} 1-m$ Cherry flies with intact serotonin level (no PCPA treatment) showed a drastic decrease in walking velocity in comparison to the heterozygous parental lines. The TRH>dTRPA1-mCherry flies with reduction in the serotonin level (PCPA treatment) revealed a significant increase in velocity in comparison to the TRH $>$ dTRPA1-mCherry flies without PCPA treatment at $32^{\circ} \mathrm{C}$. However, the walking velocity of the TRH $>$ dTRPA1-mCherry flies with PCPA treatment was still lower than in heterozygous parental controls with PCPA treatment. This was perhaps due to the fact that the reduction of the serotonin level using PCPA was partial. Hence, a complete rescue of the walking velocity of TRH>dTRPA1-mCherry flies was not observed. The partial rescue in the walking velocity of the treated TRH>dTRPA1-mCherry flies could, therefore, be the consequence of a partial reduction in the serotonin level. Furthermore, a reduction in the serotonin level caused an increase in walking velocity in the heterozygous parental lines, although the velocity difference between treated and non-treated flies was just significant in the case of TRH-Gal4 parental line but not with the UAS:dTRPA1-mCherry line. Although the effects of the reduced serotonin level due to PCPA could not be confirmed statistically for the parental lines in this experiment, the observed trend is similar to experiments in which serotonin was reduced using Shi ${ }^{(t)}$. However, the pharmacological experiments strongly suggest that the observed behavioral effects are indeed caused by an altered level of the neurotransmitter serotonin. 
A

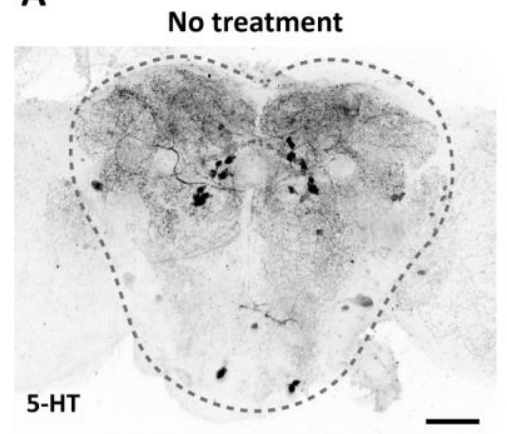

D

No treatment PCPA treatment

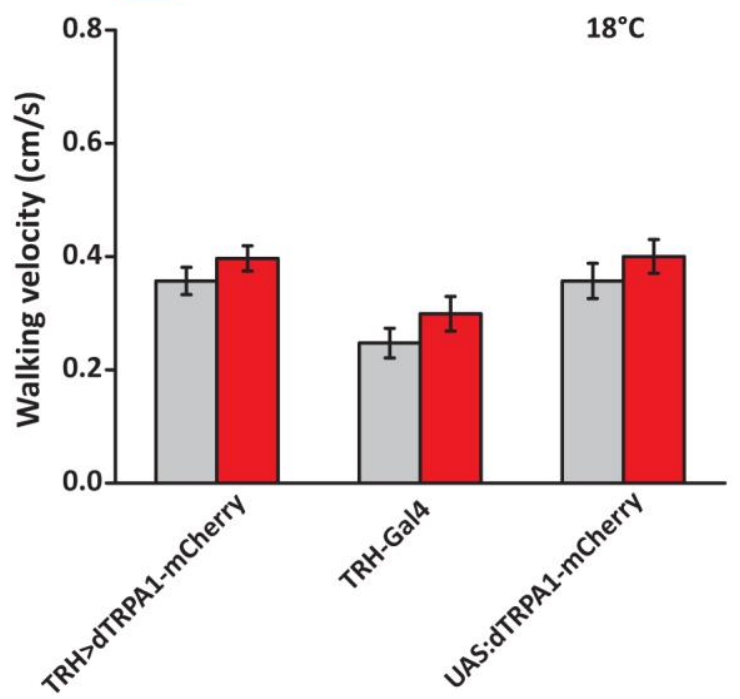

B

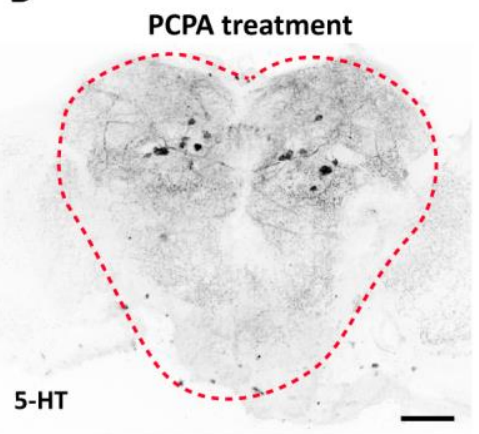

E
C

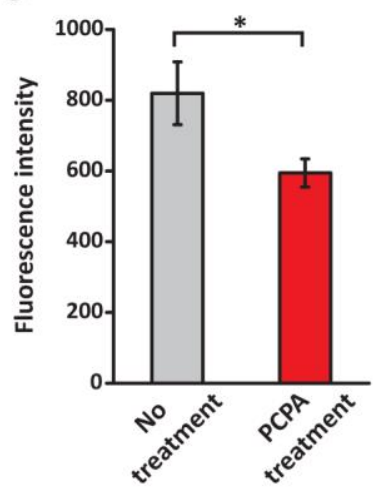

No treatment PCPA treatment

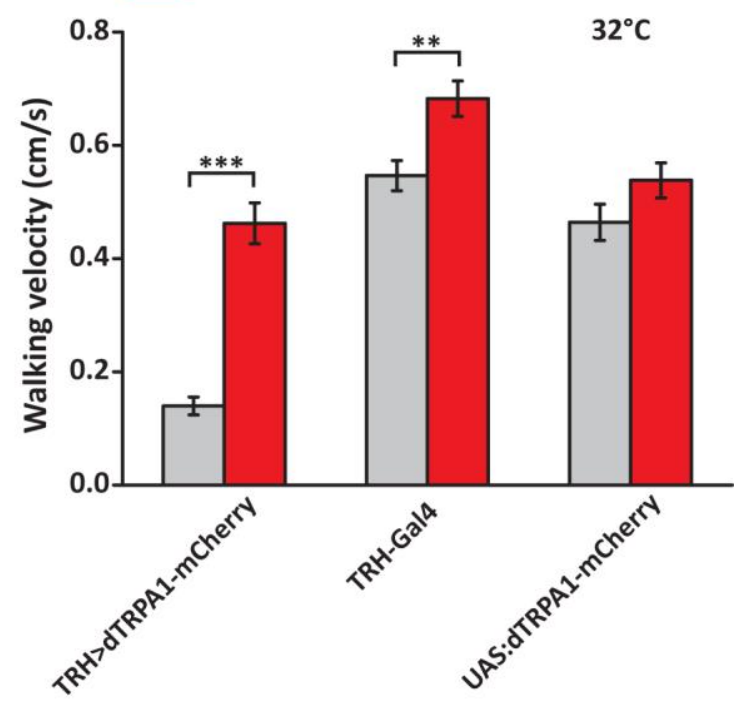

Figure 3.14. Effect of PCPA on serotonin biosynthesis and the walking velocity of flies. (A, B) The antibody staining against serotonin after PCPA treatment in the CS fly revealed a partial reduction in the serotonin level in comparison to the non-treated CS fly. The grey and red dashed borders indicate the regions used for quantification of anti-5-HT fluorescence intensity. Scale bar: $50 \mu \mathrm{M}$. (C) The intensity of anti-5-HT staining was quantified using Fiji software. The regions which were quantified are shown in the grey and red dashed lines in (A) and (B). Based on the intensity quantification, the serotonin level in the flies with PCPA treatment showed significant reduction in comparison to the flies without treatment. Two sample t-test. $\left({ }^{*}\right): P \leq 0.05 . n=5$. (D) The TRH $>d T R P A 1-m C h e r r y$ flies and the heterozygous parental lines with PCPA treatment (red bars) and without PCPA treatment (grey bars) were tested for walking velocity at $18^{\circ} \mathrm{C}$. No significant difference was observed between treated and non-treated groups. (E) The TRH>dTRPA1-mCherry flies and the heterozygous parental lines with PCPA treatment (red bars) and without PCPA treatment (grey bars) were tested for walking velocity at $32^{\circ} \mathrm{C}$. Activation of serotoninergic neurons in TRH>dTRPA1-mCherry flies with no treatment revealed significant decrease in the walking velocity in comparison to the heterozygous parental lines. The TRH>dTRPA1-mCherry flies with PCPA treatment showed a significant elevation in the walking velocity in comparison to those flies with no PCPA treatment. The walking velocity of TRH-Gal4 parental line is significantly higher in the flies with PCPA treatment in comparison to non- 
treated TRH-Gal4 flies. In the case of UAS:dTRPA1-mCherry parental line, although an increase in the velocity of the PCPA treated flies in comparison to non-treated flies was observed, but the difference was not statistically significant. One-Way ANOVA with post hoc Bonferroni correction. $\left({ }^{* *}\right): P \leq 0.01$. $\left({ }^{* *}\right): P \leq 0.001 . n=36-41$.

The next step of the study was to ask whether specific subgroups of serotoninergic neurons are responsible for the regulation of the arousal state. For this purpose a stochastic and an intersectional approach were used, the mCherry-dTRPA1 transgene was expressed in different subpopulation of serotoninergic neurons and the flies were examined in the walking velocity paradigm.

The result from the experiments above indicated a modulatory role of serotonin in adjusting the arousal level in flies. However, it was still unknown whether the modulatory effect of serotonin on the arousal level is the general effect and it can be elicited from any serotoninergic neurons or whether the regulatory effect of serotonin on arousal is evoked by certain and specific subtype of serotoninergic neurons. For investigating this question and mapping the possible serotoninergic neurons regulating arousal sate in the flies, various genetic applications including stochastic and intersectional approaches for activating restricted number of serotoninergic neurons were designed and the required tools were generated.

\subsection{Identification of serotoninergic neurons underlying the modulation of arousal state in Drosophila}

\subsubsection{Activation of restricted number of serotoninergic cells using a stochastic approach}

For expressing the mCherry-dTRPA1 transgene in a stochastic way, the FRT-FLP site directed recombination system was combined with the UAS-Gal4 system. In the FRT-FLP system, the expression of the transgene downstream of the FRT out cassette is dependent on the excision of the FRT cassette by flippase recombinase (FLP) reaction. The FRT out cassette consists of the marker gene and transcription stop sequence flanked by two direct FRT sequences.

A transgenic line carrying both the UAS sequence and the mCherry-dTRPA1 downstream of the FRT cassette was generated for this purpose. In the absence of FLP, the CD2 marker gene was expressed in Gal4 positive cells (using TRH-Gal4), but because of the transcription stop in the 
FRT cassette, mCherry-dTRPA1 was not expressed. In the case of FLP expression, excision of the FRT cassette led to the expression of mCherry-dTRPA1 in the cells which were positive for both FLP and Gal4 proteins. In this study the flippase was expressed under the control of the heatshock promoter to induce random expression of mCherry-dTRPA1 in few serotoninergic neurons. Thereby, the expression level of flippase could be manipulated by altering the duration of heat-shock. Restricted intensity and duration of heat-shocking led to a random and limited expression of FLP in few cells (Figure 3.15). In this way, investigating the effect of activating few random serotoninergic neurons (instead of activation of the entire set of serotoninergic neurons) on the arousal state was achieved. This experiment should reveal whether the effect serotoninergic cells on the arousal state is mediated by specific serotoninergic neurons or whether the activation of the whole serotonergic system is needed to reduce the animal's arousal level.

\section{No heat shock}
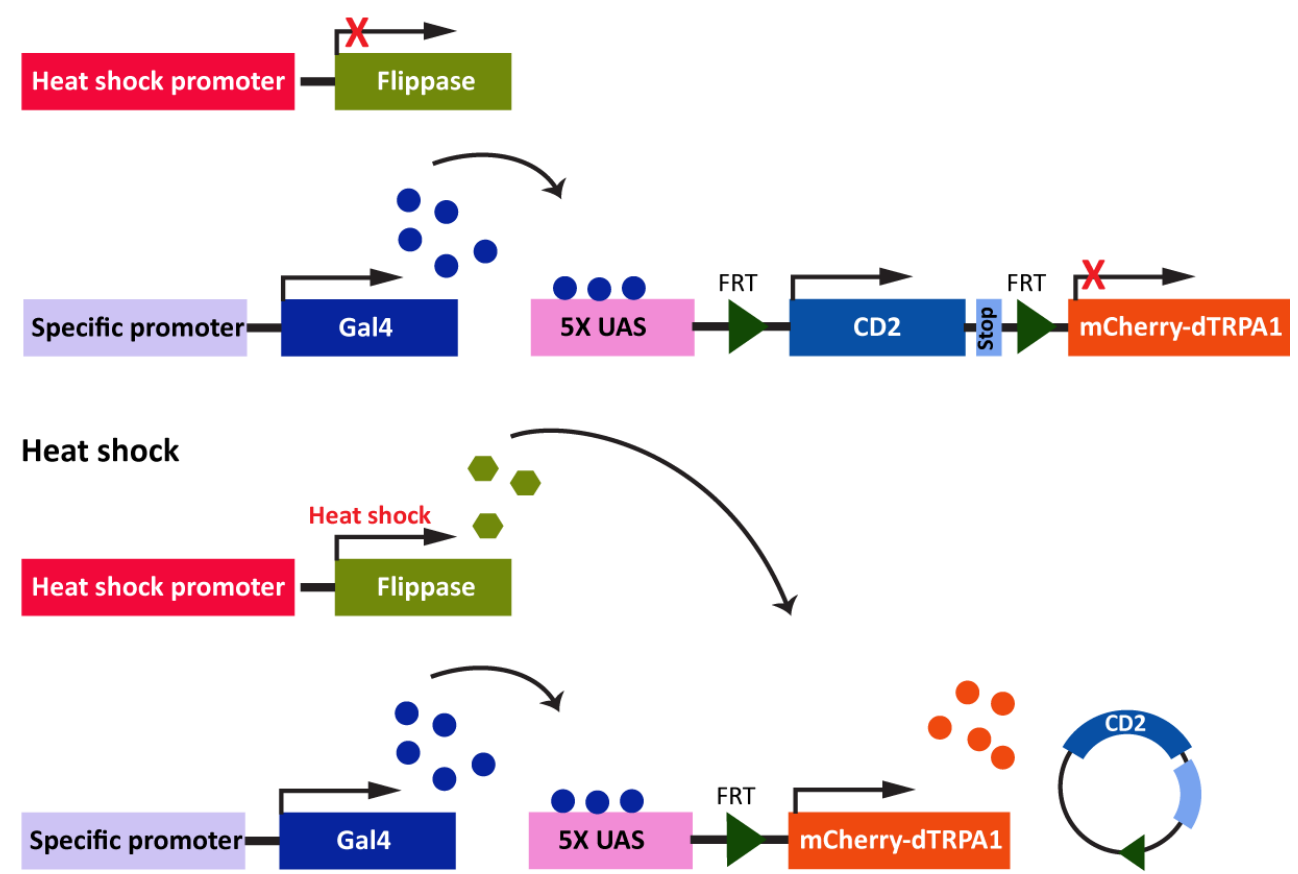

Figure 3.15. Schematic description of the stochastic expression of mCherry-dTRPA1 transgene. This approach was based on a site directed recombination system called FRT-FLP system. In the absence of any heat-shock, the flippase (FLP) under the control of heat-shock promoter was not expressed. Therefore, the CD2 gene as the marker was expressed under the control of the TRH-Gal4 driver line and transcriptional stop in the FRT cassette blocked the expression of mCherry-dTRPA1 located downstream of the cassette. Heat-induced expression of FLP led to excision of FRT cassette containing the transcription stop, therefore mCherry-dTRPA1 was expressed in the cells which were positive for FLP and Gal4. 


\subsubsection{Generating tools for stochastic gene expression}

The transgenic line expressing flippase under the control of the heat-shock promoter (hs-FLP) was kindly provided by Dr. Gary Struhl. This line was combined with different Gal4 driver lines (actin-Gal4, TRH-Gal4) to generate transgenic lines which were homozygous for FLP and Gal4 insertions. The UAS:FRT-CD2-stop-FRT-mCherry-dTRPA1 construct was generated (section 2.2.2.2 III) and sent for P-element-transgenesis to the BestGene Inc. company. Due to the random insertion of a P-element into the fly genome, four fly strains with various insertion sites were received (Table 3.4).

Table 3.4. Overview of the generated UAS:FRT-CD2-stop-FRT- mCherry-dTRPA1 lines.

\begin{tabular}{ll}
\hline UAS:FRT-CD2-stop-FRT-mCherry-dTRPA1 lines & Chromosome \\
\hline w; +; UAS:FRT-CD2-stop-FRT-mCherry-dTRPA1/TM3 (1) & 3nd \\
w; UAS:FRT-CD2-stop-FRT-mCherry-dTRPA1/gla; + (2) & $2 \mathrm{rd}$ \\
$w^{-}+$+; UAS:FRT-CD2-stop-FRT-mCherry-dTRPA1/TM3 (3) & $3 \mathrm{rd}$ \\
$w^{-}+$+; UAS:FRT-CD2-stop-FRT-mCherry-dTRPA1/TM3 (4) & $3 \mathrm{rd}$ \\
\hline
\end{tabular}

The $w^{-}$; +; UAS:FRT-CD2-stop-FRT-mCherry-dTRPA1/TM3 (3) line was not viable. For evaluating the other three transgenic lines, they were crossed to $y^{+}, w^{-}, h s-F L P ;$ actin-Gal4/cyo flies. The offspring were exposed to a heat-shock for $3 \mathrm{~h}$ at $32^{\circ} \mathrm{C}$ in a water-bath. After four days, the expression of mCherry protein was examined using a fluorescence stereo microscope. All three lines showed partial expression of mCherry in the abdomen. From this result it can be concluded that the site directed recombination mediated by FLP led to excision of FRT cassette in some cells and the mCherry-dTRPA1 was expressed in the few Gal4 and FLP positive cells in the abdomen of the flies. Therefore, using actin-Gal4 driver line, the stochastic expression was found to be functional (Figure 3.16).

Since all of the three UAS:FRT-CD2-stop-FRT-mCherry-dTRPA1 lines expressed the mCherrydTRPA1 in the random manner under the control of the actin-Gal4 driver line and none of these three lines had leaky expression without being crossed to the Gal4, the most healthy line in terms of laying more eggs and propagating faster, $w^{-}$; UAS:FRT-CD2-stop-FRT-mCherrydTRPA1/gla;+ (2), was chosen for the following experiments. 

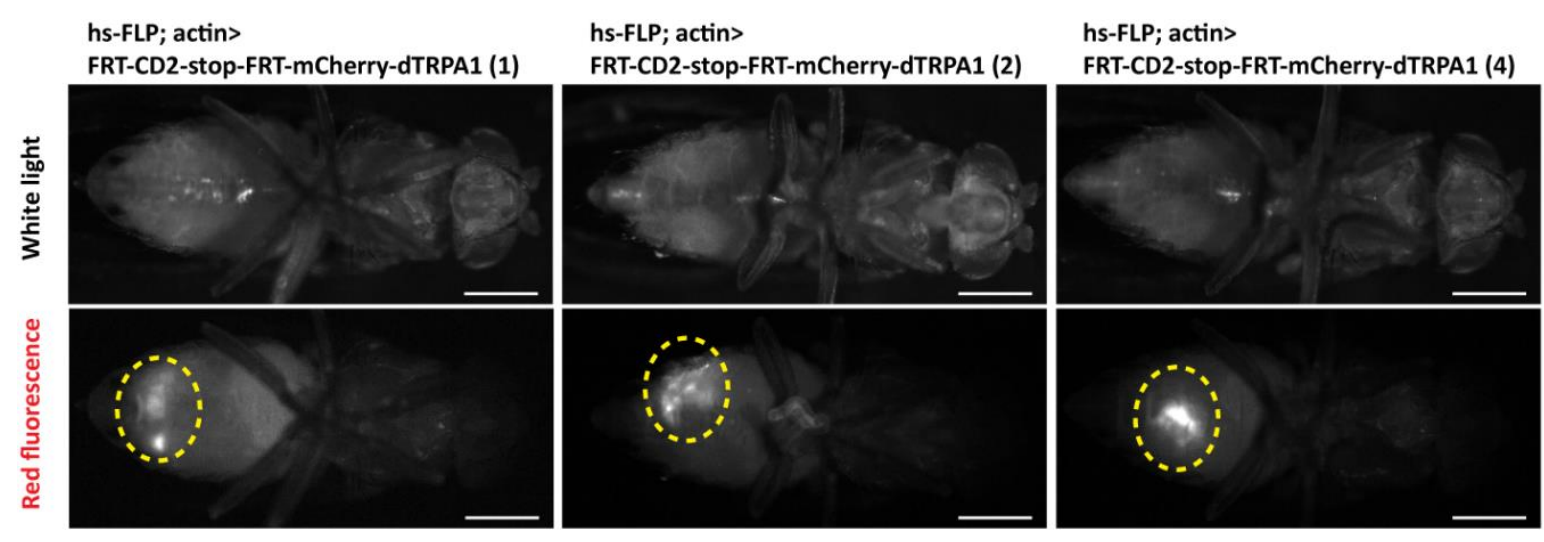

Figure 3.16. Stochastic expression of mCherry-dTRPA1 under the control of an actin-Gal4 driver line. The three UAS:FRT-CD2-stop-FRT-mCherry-dTRPA1 strains were crossed to the hs-FLP; actin-Gal4 line. In the hs-FLP; actin> Frt-CD2-stop-FRT-mCherry-dTRPA1 flies the expression of the flippase was induced by $3 \mathrm{~h}$ heat-shock at $32^{\circ} \mathrm{C}$.The images in the first row were obtained using white light for visualizing the flies. The second row of images were obtained using mCherry excitation light $(510 \mathrm{~nm})$ which show the stochastic expression of mCherry-dTRPA1 under the control of actin promoter. The yellow circles indicate the regions with mCherry-dTRPA1 expression. Scale bar: $500 \mu \mathrm{m}$.

For evaluating the stochastic expression of the mCherry-dTRPA1 transgene in the serotoninergic neurons, w; UAS:FRT-CD2-stop-FRT-mCherry-dTRPA1/gla; + flies were crossed with $y^{+}, w^{-}, h s-F L P ; T R H-G a l 4$. For expressing the transgene in just few serotoninergic cells, different protocols for heat-induction of FLP were tested. Since the LP cluster of serotoninergic neurons develops in the adult flies (Valles and White, 1988), the heat-shocking of the flies was performed after eclosion for various durations at $32^{\circ} \mathrm{C}$. The expression pattern of mCherrydTRPA1 in 5-6 days old flies, which were kept at $18^{\circ} \mathrm{C}$ before and after heat-shock, was visualized using immunohistochemistry against RFP and 5-HT. Some expression of mCherry was observed in the flies even without heat-shock, probably due to some expression of the hsflippase even at low temperatures. The expression level of mCherry-dTRPA1 increased with inducing more flippase expression (Figure 3.17). For expressing the transgene in relatively few serotoninergic cells, a $1 \mathrm{~h}$ heat-shock protocol was used for the following experiments. 


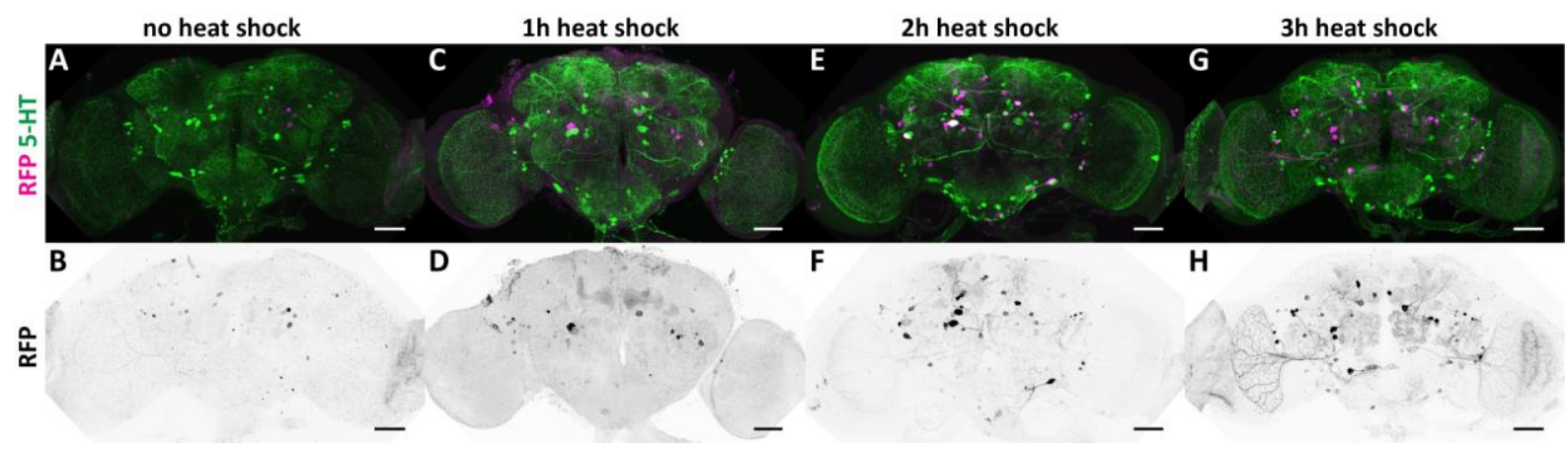

Figure 3.17. Stochastic expression of mCherry-dTRPA1 under the control of TRH promoter using different duration of heat induction. The expression pattern was visualized by immunohistochemistry using RFP antibody against mCherry protein and 5-HT antibody. First row shows the merge images of RFP (magenta) and 5HT (green) staining and the images in the second row show the RFP staining (black). The flippase expression was induced by (A, B) no heat-shock, (C, D) 1 h heat-shock, $(E, F) 2$ heat-shock and $(G, H) 3$ heat-shock in the hs-FLP; TRH>Frt-CD2stop-FRT-mCherry-dTRPA1 flies. Various amount of flippase induction led to different amounts of mCherry-dTRPA1 expression under the control of TRH-Gal4 driver line. Scale bar: $50 \mu \mathrm{m}$.

\subsubsection{The effect of thermoactivating subsets of serotoninergic neurons on the arousal level}

The hs-FLP; TRH>Frt-CD2-stop-FRT-mCherry-dTRPA1 flies with stochastic expression of mCherry-dTRPA1 channel were tested in the single fly assay for walking velocity at $32^{\circ} \mathrm{C}$. The flies were given heat-shock for $1 \mathrm{~h}$ at $32^{\circ} \mathrm{C}$ after eclosion and then transferred back to $18^{\circ} \mathrm{C}$. The expression pattern of the dTRPA1 channel was random and variable between flies (Figure 3.18). In order to determine the possible correlation between activation of cells in specific serotoninergic clusters and the reduction in the velocity as an indicator for the altered arousal level, the mCherry-dTRPA1 expression pattern was determined for each individual fly after the behavioral test. For this purpose, after measuring the walking velocity of 5-6 days old female flies which expressed mCherry-dTRPA1 in random subset of serotoninergic neurons, the brains were dissected and immunostained using anti-RFP and anti-5-HT antibodies. As described in section 3.2.2, the TRH-Gal4 line covers $79 \pm 12$ (mean \pm SD) serotoninergic cells and $168 \pm 12$ (mean \pm SD) non-serotoninergic cells, and it is to note that in most cases some expression also was observed in non-serotoninergic cells. In total, the walking velocity and the expression pattern of mCherry-dTRPA1 within serotoninergic neurons were determined in 256 individual flies. Some examples of the stochastic expression pattern of mCherry-dTRPA1 are shown in figure 3.18 . 

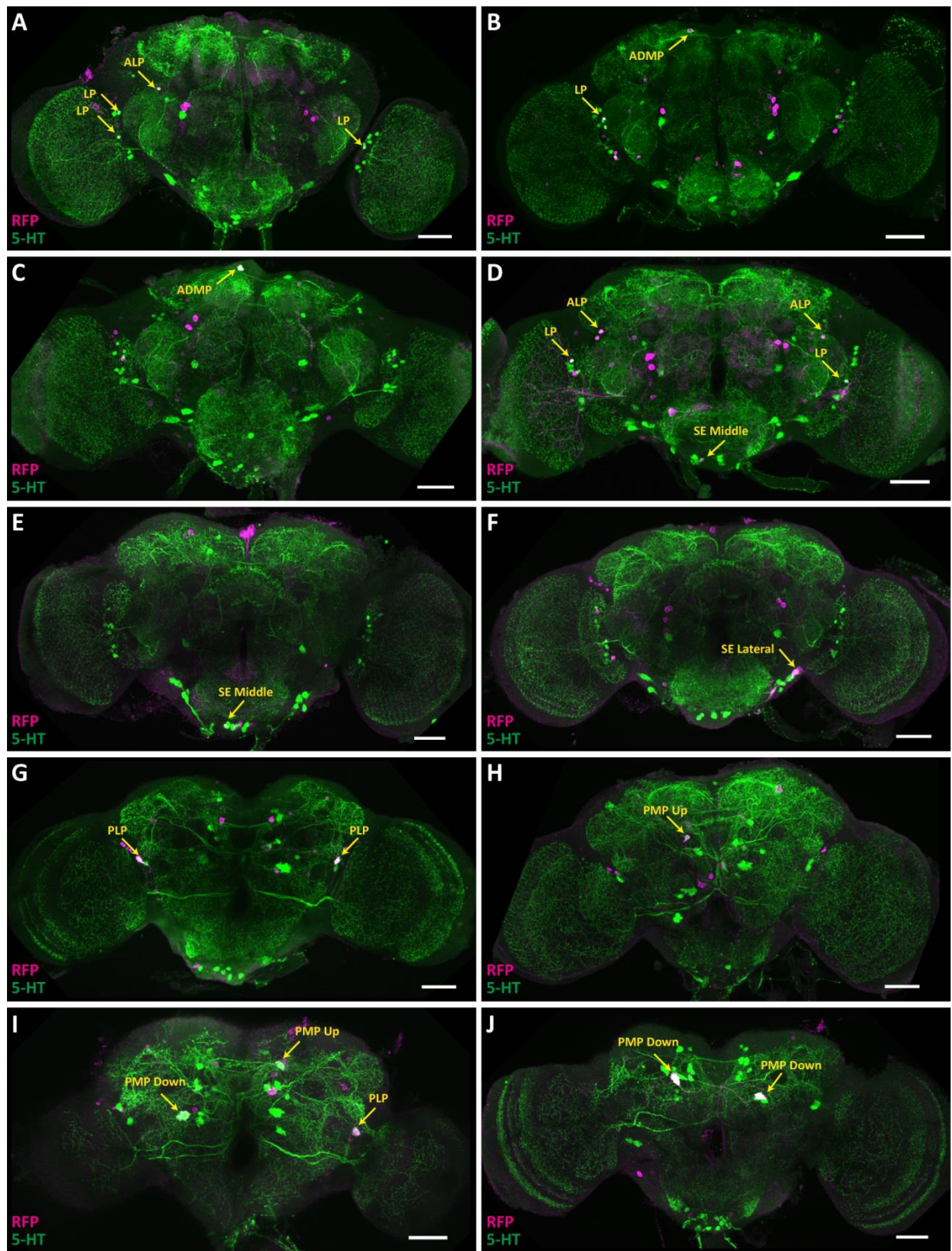

Figure 3.18. Stochastic expression of mCherry-dTRPA1 in different subsets of serotoninergic neurons. The expression pattern of mCherry-dTRPA1 in the hs-FLP; TRH>Frt-CD2-stop-FRT-mCherry-dTRPA1 flies were determined using double-staining against mCherry (RFP antibody, magenta) and serotonin (5-HT, green). The white color indicates the serotoninergic cells expressing mCherry-dTRPA1. Scale bar: $50 \mu \mathrm{m}$. 
For investigating whether the arousal level in the flies is dependent on the number of activated serotoninergic neurons, the relation between the numbers of serotoninergic cells expressing mCherry-dTRPA1 and the walking velocity in flies was analyzed. No significant differences between the walking velocity of the flies with the determined number of activated serotoninergic neurons was found (Figure 3.19). However, a slight trend of a decreased velocity in the files expressing mCherry-dTRPA1 in more than 10 cells was observed. Such a slight trend would be expected even if single cells mediate the behavioral change, since the probability that those cells are included in the expression pattern increases with an increasing total number of targeted cells. To address the question in more detail, the information from the 256 flies (expression pattern and velocity) was plotted separately for each cluster (Figure 3.20). For the PMP middle and the LP clusters, a slight trend of decreasing velocity with increase in the number of activated cells in the cluster was observed (Figure 3.20 C, I). However, only for the PMP Down cluster a significant difference in the walking velocity of the flies with different number of the cells expressing mCherry-dTRPA1 was found (Figure $3.20 \mathrm{~J}$ ). For the other seven clusters (ALP, AMP, ADMP, Se Lateral, SE Middle, PLP and PMP Up) no significant change in velocity with increasing number of activated cells was observed (Figure 3.20). 


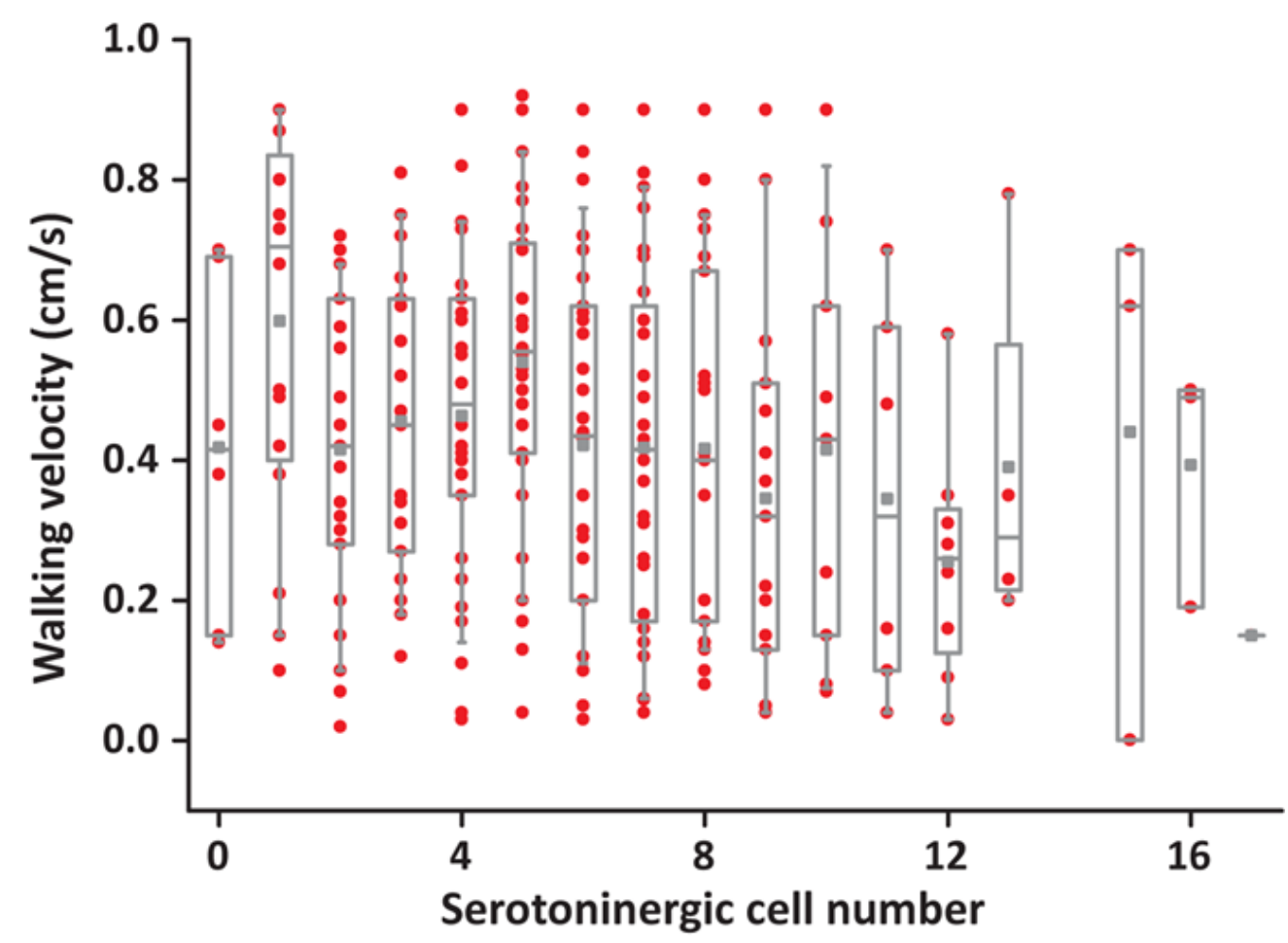

Figure 3.19. Effect of the number of activated serotoninergic cells on the walking velocity. Based on the result of walking velocity assay and immunostaining for mapping the mCherry-dTRPA1 expression pattern in hs-FLP; TRH>FRT-CD2-stop-FRT-mCherry-dTRPA1 flies, no significant difference between velocity of the flies expressing mCherry-dTRPA1 in different number of serotoninergic neurons was found. Each red dot indicates the walking velocity and the mCherry-dTRPA1 expression pattern for each fly. The box plots (gray) indicate the interquartile range, mean and median velocity for each group of the flies with distinct number of the serotoninergic neurons expressing mCherry-dTRPA1. The whiskers indicate 10-90 percentiles. Kruskal-Wallis Anova. $n=256$. 
A

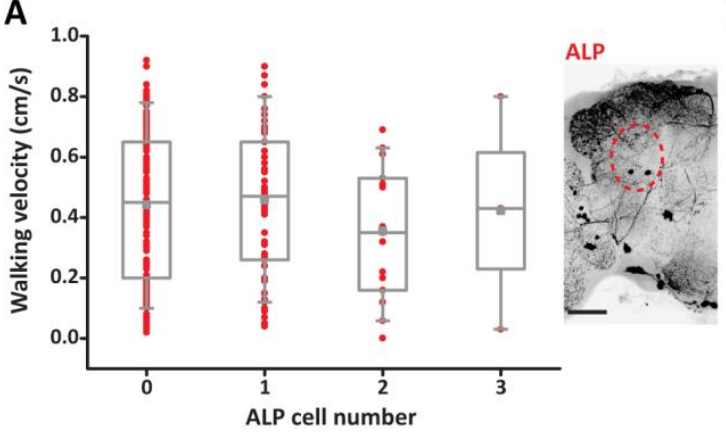

C

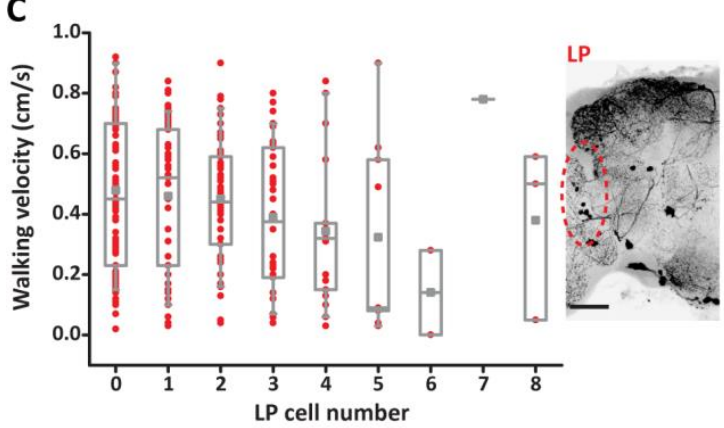

E

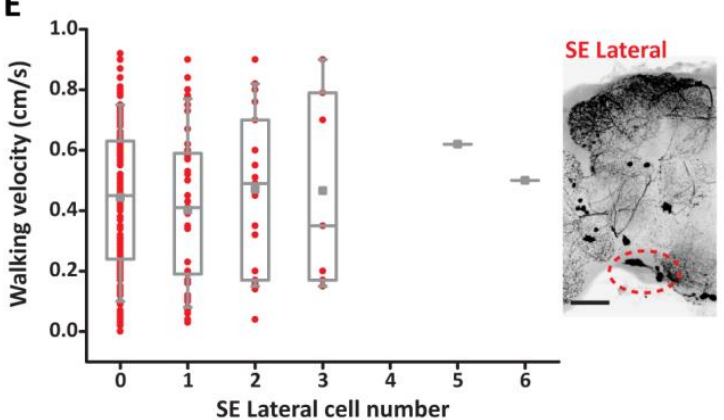

G
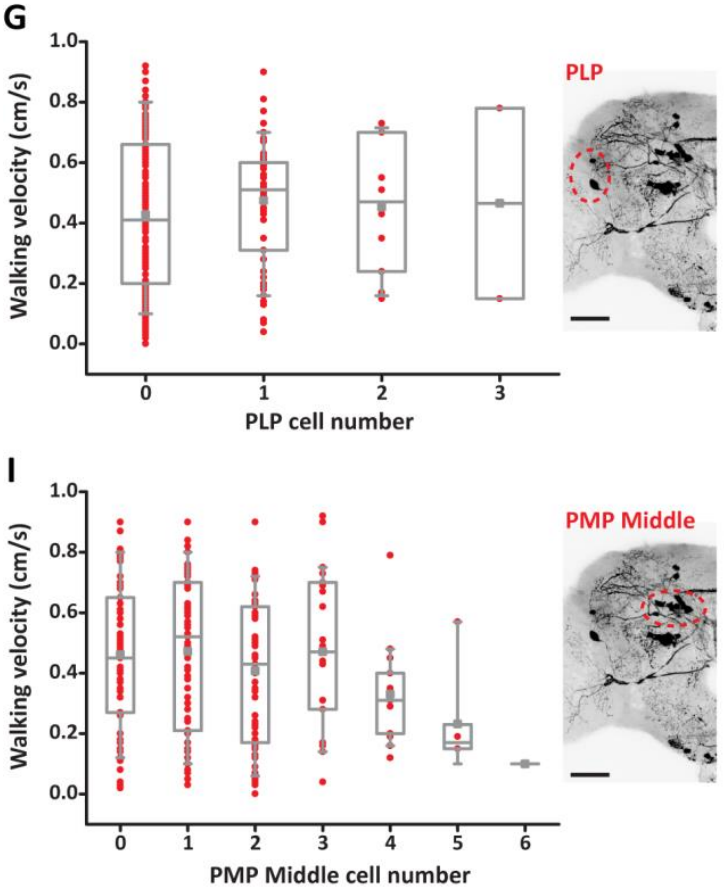

B

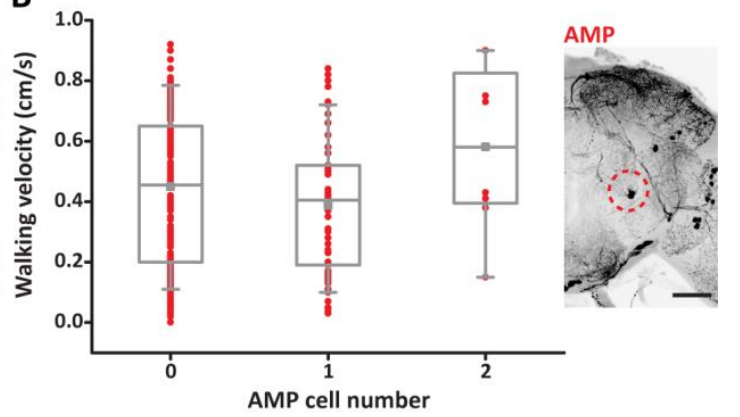

.
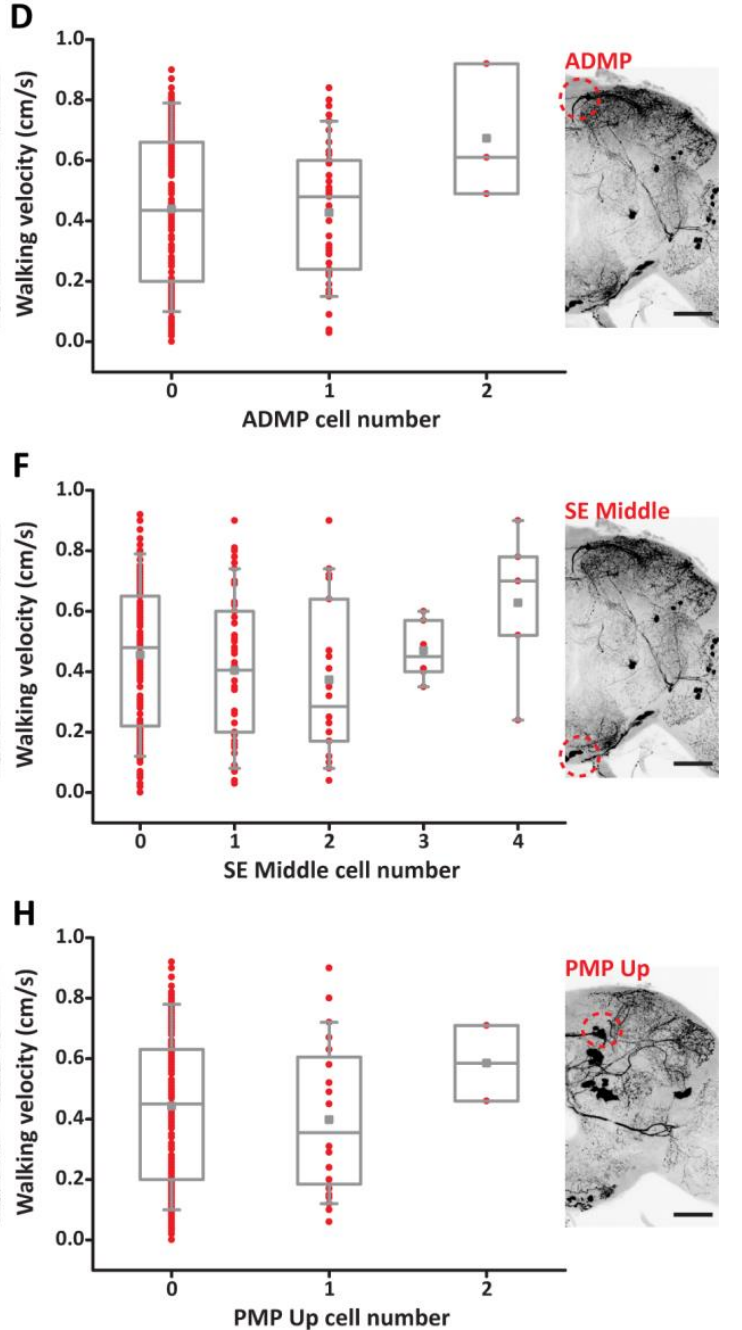

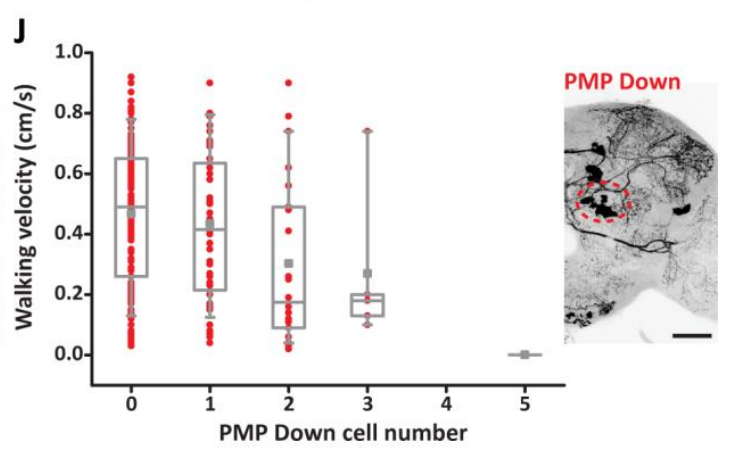


Figure 3.20. Effect of the number of activated serotoninergic cells in specific clusters on the walking velocity. Based on the result of walking velocityn assay and immunostaining for mapping the mCherry-dTRPA1 expression pattern in hs-FLP; TRH>Frt-CD2-stop-FRT-mCherry-dTRPA1 flies, the walking velocity of each fly with determined number of activated cells in each cluster was plotted. The activation of the serotoninergic cells in (A) ALP cluster, (B) AMP cluster, (C) LP cluster, (D) ADMP cluster, (E) SE Lateral cluster, (F) SE Middle cluster, (G) PLP Cluster, (H) PMP Up cluster and (I) PMP Middle cluster did not significantly change the walking velocity of the flies. However a slight trend of decreasing velocity due to increase in the number of activated cells in (C) LP cluster and (I) PMP Middle cluster was observed. For (J) PMP Down cluster, a significant difference in the walking velocity of the flies with different number of the cells expressing mCherry-dTRPA1 was found $(P=0.006)$. Each red dot indicates the walking velocity and the mCherry-dTRPA1 expression pattern for each fly. The position of each specific cluster is shown with red circles in the brain which was immunostained using anti-5-HT antibody. Scale bar: 50 $\mu$ m. The box plots (gray) indicate the interquartile range, mean and median velocity for each group of the flies with distinct number of the serotoninergic neurons in each cluster expressing mCherry-dTRPA1. The whiskers indicate 10-90 percentiles. The data set for each plot is the information obtained from one group of the flies $(n=256)$ expressing mCherry-dTRPA1 stochastically in different subsets of neurons. Kruskal-Wallis Anova.

For a further analysis of a potential effect of specific serotoninergic clusters on the arousal state of the fly, the velocity of the flies having no mCherry-dTRPA1 expression in the specific clusters was compared to the velocity of the flies expressing mCherry-dTRPA1 in one or more cell(s) in those clusters (Figure 3.21). Again, a significant difference between the flies with and without mCherry-dTRPA1 expression was found only in the PMP Down cluster (Figure 3.21). In all other clusters, the velocity of the flies with mCherry-dTRPA1 expression was not significantly different from the flies without any expression in cells of those clusters. 


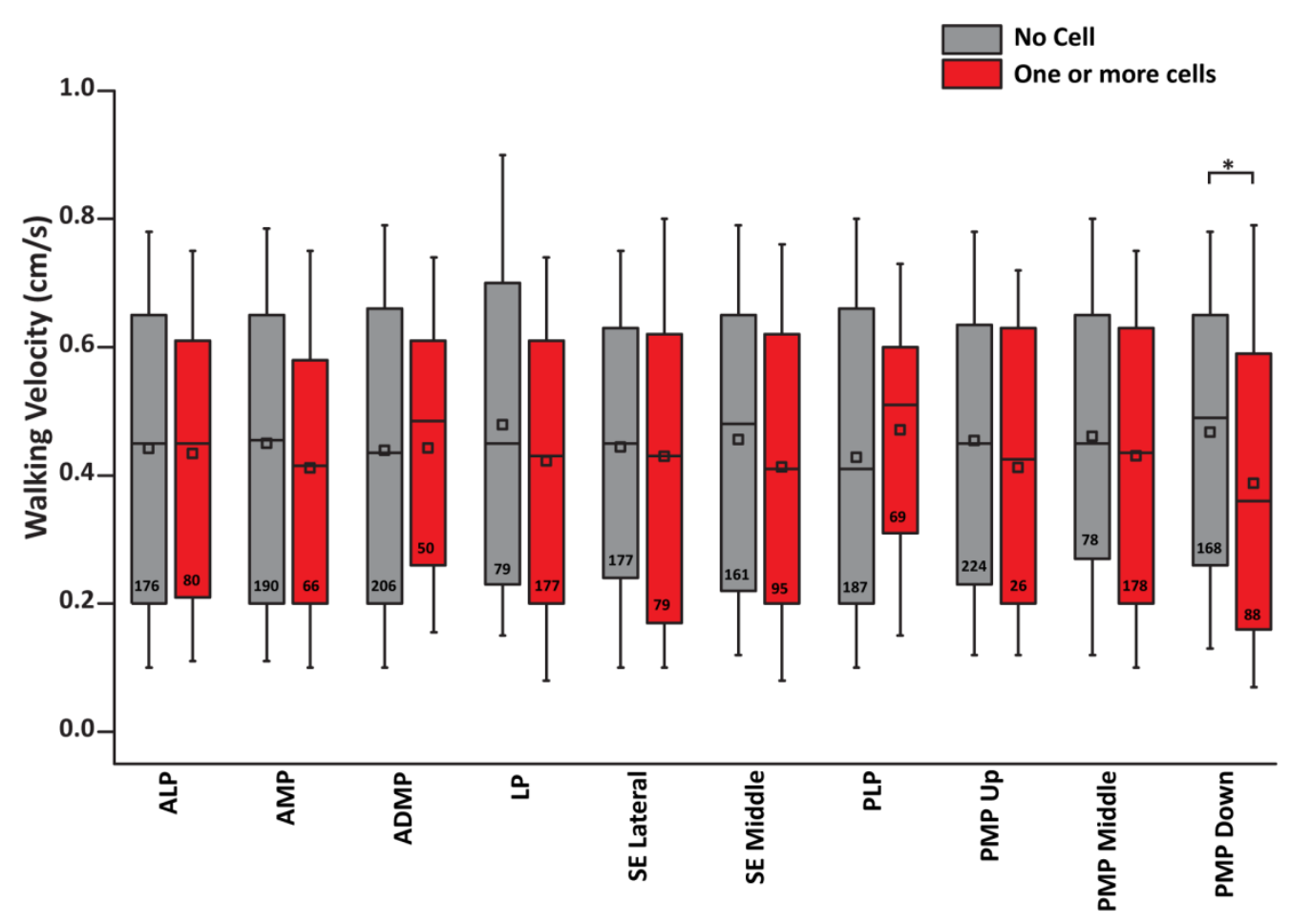

Figure 3.21. Comparison of the velocity of the flies with and without expression of mCherry-dTRPA1 in the specific clusters. The velocity of the flies with one or more activated cell(s) in PMP Down cluster was significantly less in comparison to the flies with no cell activity in this cluster. The box plots indicate the interquartile range, mean and median velocity. The sum of the sample size for red and gray box plots for each cluster was 256 flies. The sample size for each box plot is indicated inside the box. Mann-Whitney Test. $\left({ }^{*}\right)$ : $P \leq 0.05$.

The results of this experiment highlight the PMP Down cluster as the strongest candidate for a certain cluster that modulates the arousal state. For a more detailed analysis of the effect of this cluster on the arousal level, the velocity of the flies having mCherry-dTRPA1 expression in different numbers of the PMP Down cells $(0,1$ or 2$)$ were compared. A significant difference in the velocity of the flies with mCherry-dTRPA1 expression in two cells in comparison to no cell and one cell was found (Figure 3.22 A). In other words, the flies expressing mCherry-dTRPA1 in at least two PMP Down cells showed a significant reduction in the walking velocity. This observation was further confirmed by comparing the distribution of velocity of the flies expressing mCherry-dTRPA1 in one, two and no cells of the PMP Down cluster (Figure $3.22 \mathrm{~B}$, C). Since the number of the flies with mCherry-dTRPA1 expression in three or more cells in this cluster was low, no statistical comparison for these flies was performed. 
A

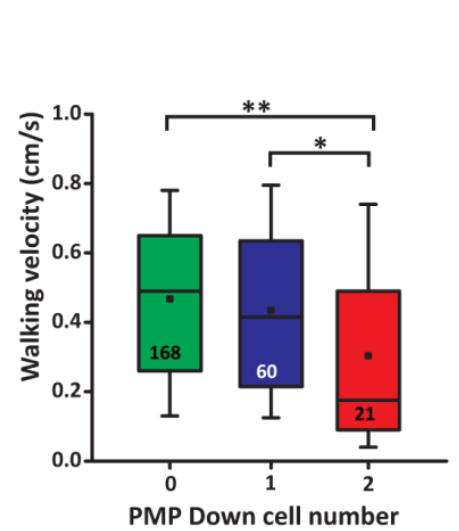

B

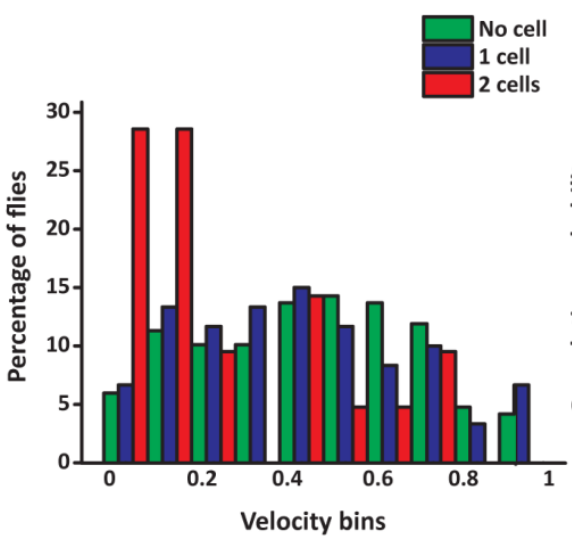

C

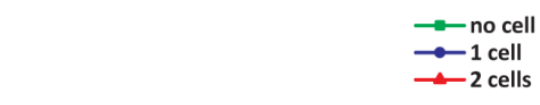

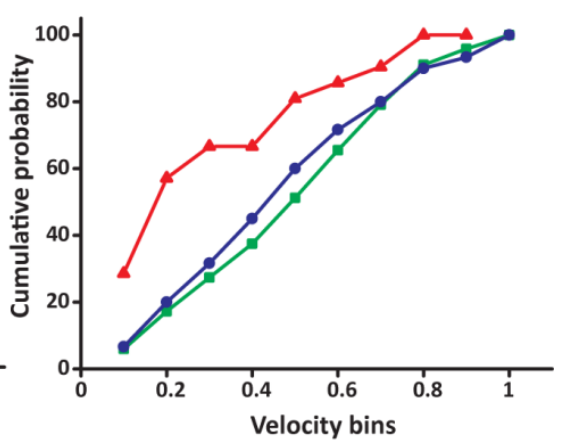

Figure 3.22. Comparison of the velocity across the flies expressing mCherry-dTRPA1 in no cell, 1 cell and $\mathbf{2}$ cells in the PMP Down cluster. (A) A significant difference in the velocity of the flies with mCherry-dTRPA1 expression in two cells in comparison to no cell and one cell was found. The box plots indicate the interquartile range, mean and median velocity. The sample sizes are indicated in the boxes. Mann-Whitney Test. $\left({ }^{*}\right): P \leq 0.05,\left({ }^{* *}\right): P \leq 0.001$. (B) The distribution of the percentage of the flies expressing mCherry-dTRPA1 in one, two and no cells of the PMP Down cluster in velocity bins of $0.1 \mathrm{~cm} / \mathrm{s}$ interval. (C) The cumulative probability of the flies expressing mCherrydTRPA1 in one, two and no cells of the PMP Down cluster in velocity bins of $0.1 \mathrm{~cm} / \mathrm{s}$ interval.

Although the result of the stochastic experiment indicates a significant role of the PMP Down cluster in modulating the arousal state in the flies, it still remained unclear if all the cells or specific cells in this cluster were sufficient for decreasing the arousal level.

\subsubsection{An intersectional approach for expressing mCherry-dTRPA1 in different subsets of serotoninergic neurons}

In order to restrict the expression of the mCherry-dTRPA1 reproducibly in a specific subset of serotoninergic neurons (not in a random manner), an intersectional approach (section 1.3.4), was designed and the required tools were generated. By using this approach, investigating the possible role of the PMP down cluster (as the best candidate based on the stochastic study) on regulation of arousal level in the flies was achieved. In the designed experiment, the FLP-FRT and UAS-Gal4 systems were combined, in a way that the expression of mCherry-dTRPA1 was restricted to the overlapping domain of two promoters which drive FLP and Gal4 expression. In the intersectional approach the FLP was expressed under the control of the TRH promoter. Various Gal4 driver lines with different expression pattern including different subsets of serotoninergic neurons were required for this approach. For this purpose some Gal4 lines from the Janelia-Farm Gal4 stock collection (Jenett et al., 2012) were screened and selected for 
anatomical and behavioral experiments (section 3.7.2.1.). Therefore, by combining UAS:FRTCD2-stop-FRT-mCherry-dTRPA1, Janelia Gal4 line and TRH-FLP, the expression of the mCherrydTRPA1 was restricted to the serotoninergic cells which were covered by both Gal4 and FLP promoters (Figure 3.23).

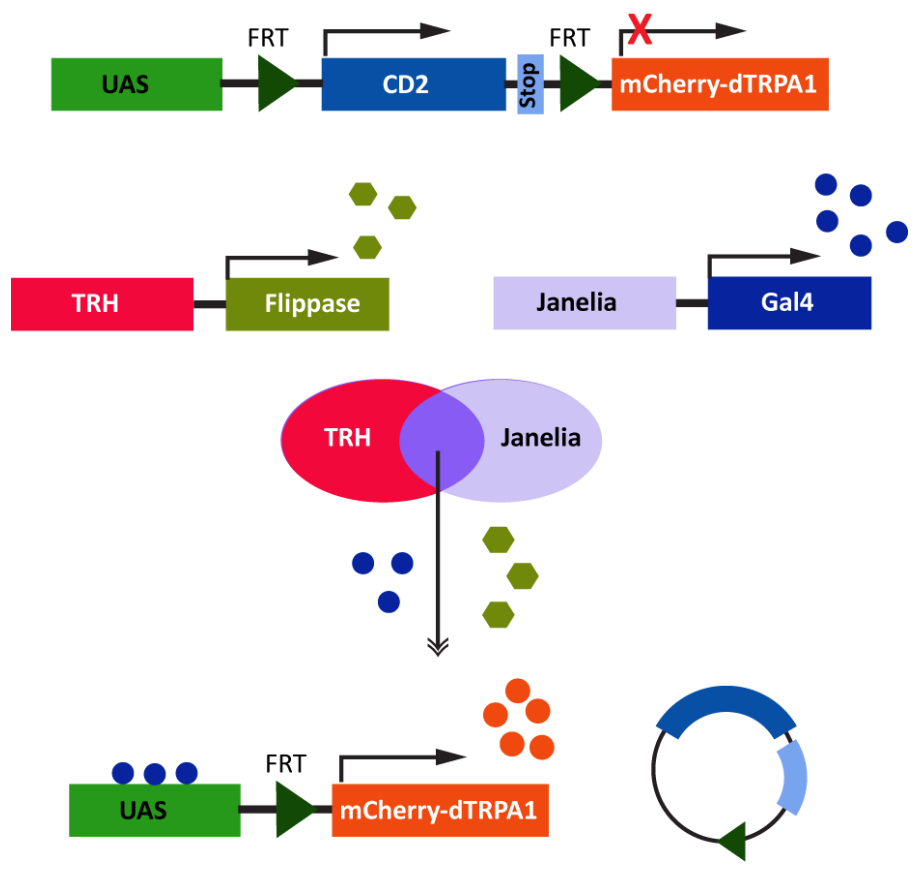

Figure 3.23. Schematic description of the intersectional expression of mCherry-dTRPA1 transgene. By combining the FLP-FRT and UAS-Gal4 systems, the expression of the mCherry-dTRPA1 was restricted to the overlapping domain of TRH and Janelia promoters.

\subsubsection{Developing the required tools for intersectional expression}

Since the designed intersectional approach was based on the FLP-FRT system, UAS:FRT-StopFRT-(2x)mCD8:GFP (Yu et al., 2010) and UAS:FRT-CD2-stop-FRT-mCherry-dTRPA1 (described in in the stochastic approach, section 3.7.1.1) the required fly lines were used for anatomical and behavioral study, respectively. Instead of heat-induced FLP (hs-FLP), which was used in the stochastic approach, a line with spatial defined expression pattern of FLP in the serotoninergic neurons was required. The TRH-flippase-IRES-flippase (TRH-FIF) construct consisting of two copies of the flippase cDNA was generated and sent to BestGene Inc. company for PhiC31 integrase-mediated transgenesis in the second and third chromosomes (section 2.2.2.2 IV). The expression and functionality of these two lines were determined by crossing them to the actinFRT-Stop-FRT-Gal4; UAS:GFP as the FLP reporter line (Pignoni and Zipursky, 1997). In the 
progeny of the cross, the GFP was expressed under the control of actin promoter due to excision of FRT stop cassette upstream of the Gal4 in the FLP positive cells. Using immunohistochemistry against GFP and 5-HT, the expression pattern of the flippase was verified in each serotoninergic cell cluster (Figure 3.24; Table 3.5).

actin-FRT-Stop-FRT-Gal4; UAS:GFP; TRH-FIF III

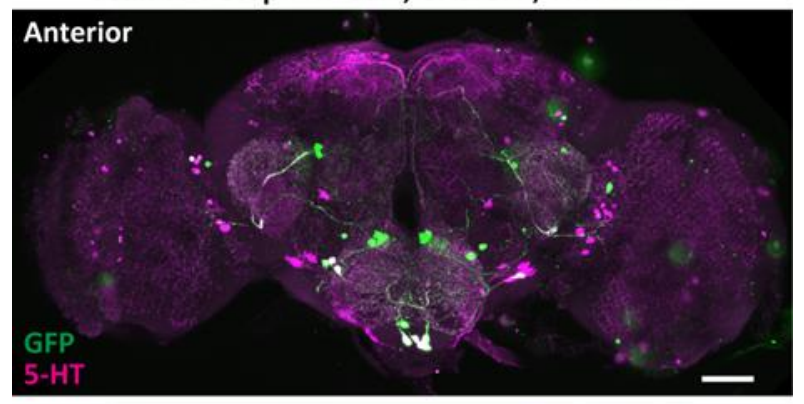

Figure 3.24. Visualizing the expression pattern of a TRH FIF line using actin-FRT-Stop-FRT-Gal4; UAS:GFP as the FLP reporter line. The anterior and posterior views of the Drosophila brain expressed GFP under the control of

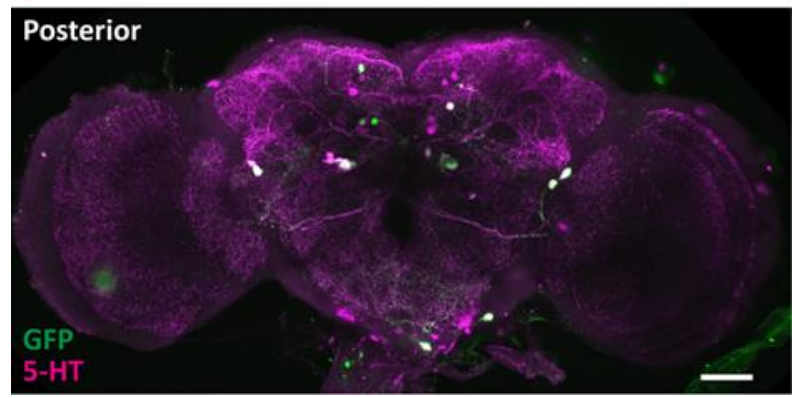
actin promoter in the FLP positive cells. The expression pattern was visualized using GFP (green) and 5-HT antibody antibodies (magenta). The staining in white color indicates the serotoninergic cells expressing FLP. Scale bar: $50 \mu \mathrm{m}$. 
Table 3.5. Number of the serotoninergic cells expressing flippase in actin-FRT-Stop-FRT-Gal4; UAS:GFP, TRH-FIF flies. The number of serotoninergic cells and non-serotoninergic cells (unspecific) expressing GFP were counted (mean $\pm S D$ ). Since the expression of the GFP was dependent on the expression of flippase in the cell, the expression pattern of the FIF protein from the GFP expression was obtained. $n=3$ entire brains.

\begin{tabular}{lcc}
\hline $\begin{array}{l}\text { 5-HT } \\
\text { clusters }\end{array}$ & $\begin{array}{c}\text { TRH-FIF (II), } \\
\text { FLP reporter }\end{array}$ & $\begin{array}{c}\text { TRH-FIF (III), } \\
\text { FLP reporter }\end{array}$ \\
\hline ALP & $0 \pm 0$ & $1 \pm 0$ \\
AMP & $0 \pm 0$ & $0 \pm 0$ \\
ADMP & $0 \pm 0$ & $0 \pm 0$ \\
LP & $0 \pm 0$ & $6 \pm 1$ \\
SE Lateral & $6 \pm 2$ & $8 \pm 4$ \\
SE Middle & $6 \pm 2$ & $8 \pm 1$ \\
PLP & $2 \pm 1$ & $4 \pm 0$ \\
PMP up & $2 \pm 2$ & $2 \pm 0$ \\
PMP middle & $3 \pm 1$ & $14 \pm 0$ \\
PMP down & $4 \pm 2$ & $5 \pm 1$ \\
Sum (specific) & $23 \pm 4$ & $34 \pm 5$ \\
Unspecific & $10 \pm 1$ & \\
\hline
\end{tabular}

In addition to the UAS:FRT and FLP lines, the Gal4 driver lines, which cover a restricted number of serotoninergic neurons in the PMP down cluster, were required for the intersectional approach. For this purpose, $\sim 500$ Gal4 lines were prescreened for potential targeting of serotoninergic cells, using the available images (immunostaining images of the Gal4 lines expressing GFP) in the website (http:// flystocks.bio.indiana.edu/ Browse /gal4 /gal4_Janelia.php). After the prescreening step, 65 different lines were ordered from the Bloomington stock center and screened for the expression in serotoninergic cells using doubleimmunostaining. In order to screen these 65 lines, they were crossed to UAS:cameleon (as the 
green reporter line, Fiala et al., 2002) and the expression pattern visualized using immunostaining. 30 lines were found to show expression in at least one serotoninergic neuron. Some examples of this screen are shown in figure 3.25 .

In order to verify the intersectional approach for expressing the transgene in subsets of serotoninergic neurons, TRH-FIF (II) and UAS:FRT-stop-FRT-(2x)mCD8:GFP (III) lines were combined to generate a stable transgenic line which was homozygous for both FLP and UAS insertions. To determine the expression of GFP in the defined serotoninergic cells, w; TRHFIF/cyo; UAS:FRT-stop-FRT-(2x)mCD8:GFP/TM3 line was crossed to different Gal4 lines, and then the dissected brains of the offspring were immunostained using GFP and serotonin antibodies. The expression patterns after intersectional expression were compared to Gal4 expression patterns (using UAS:cameleon) in figure 3.25. In this way, the expression of the transgene in a limited number of the serotoninergic cells was achieved. 


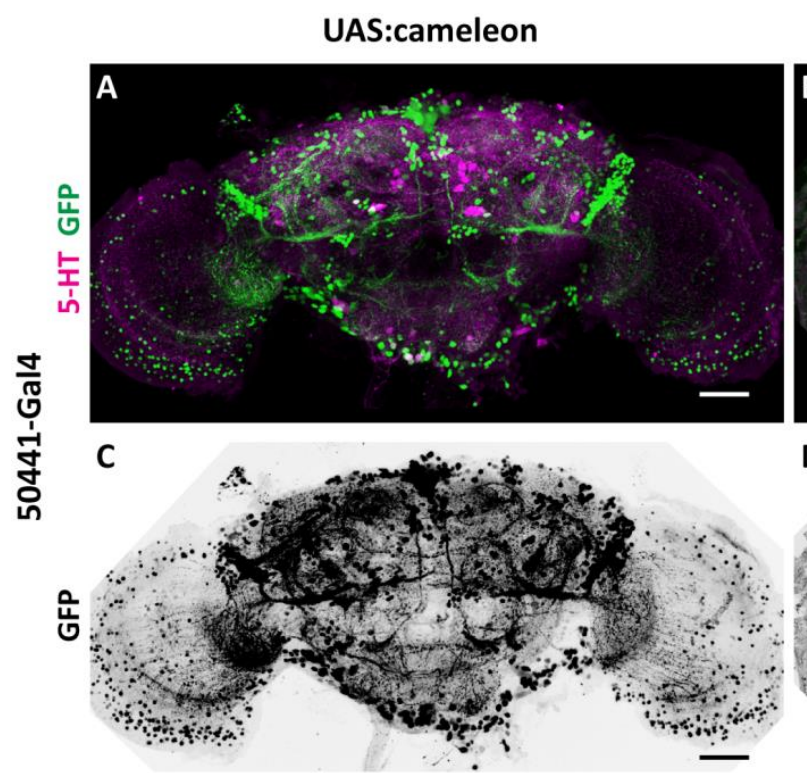

TRH-FIF; UAS:FRT-stop-FRT-mCD8:GFP
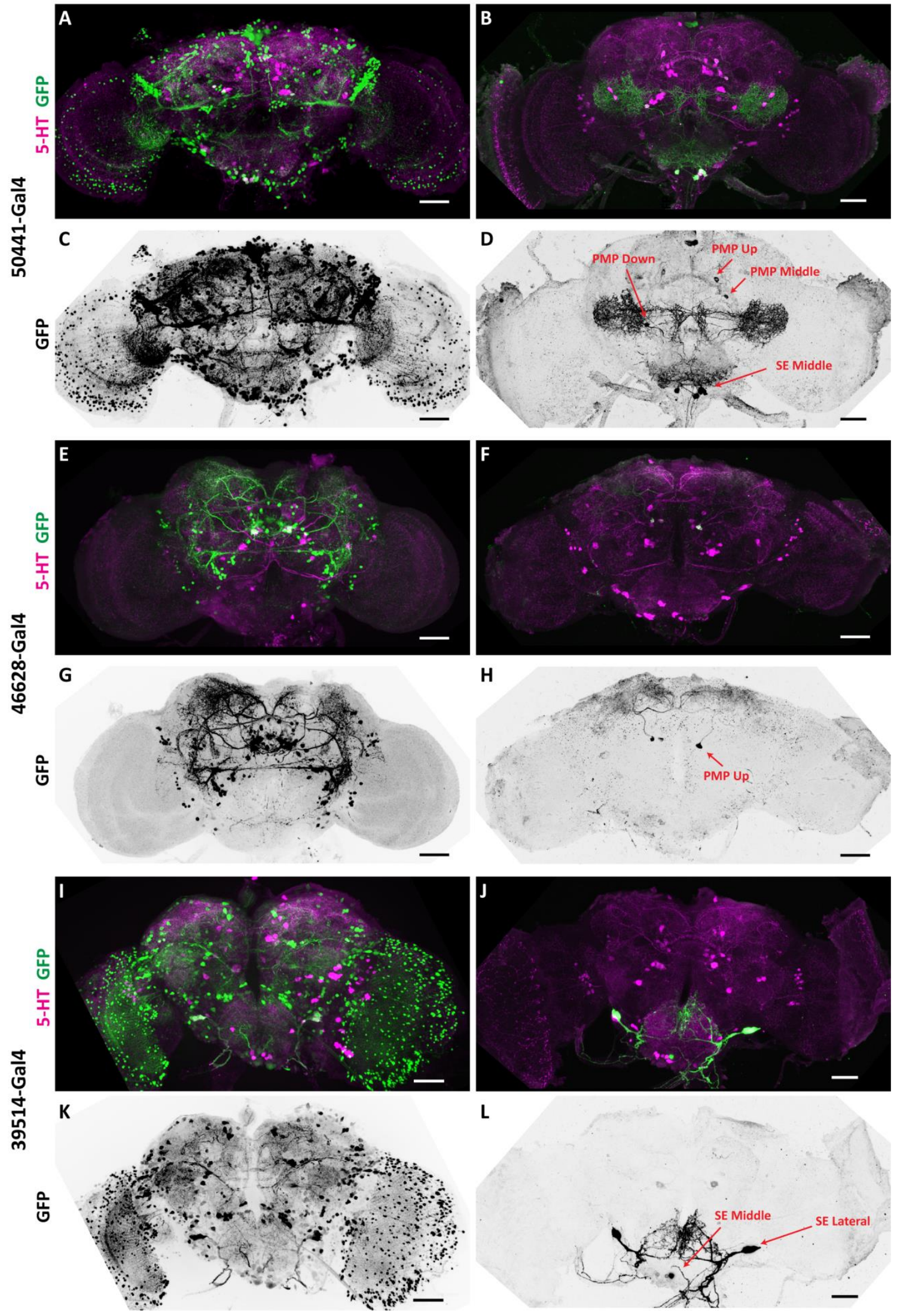

L

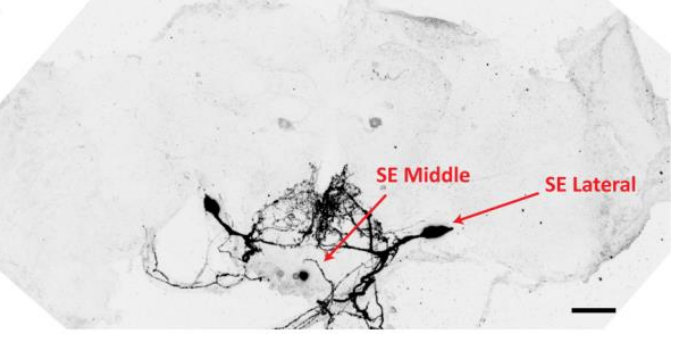




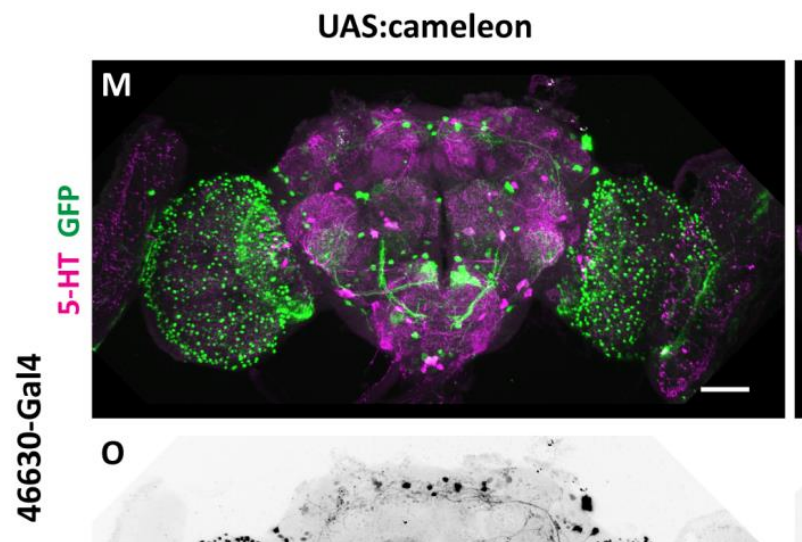

TRH-FIF; UAS:FRT-stop-FRT-mCD8:GFP

:
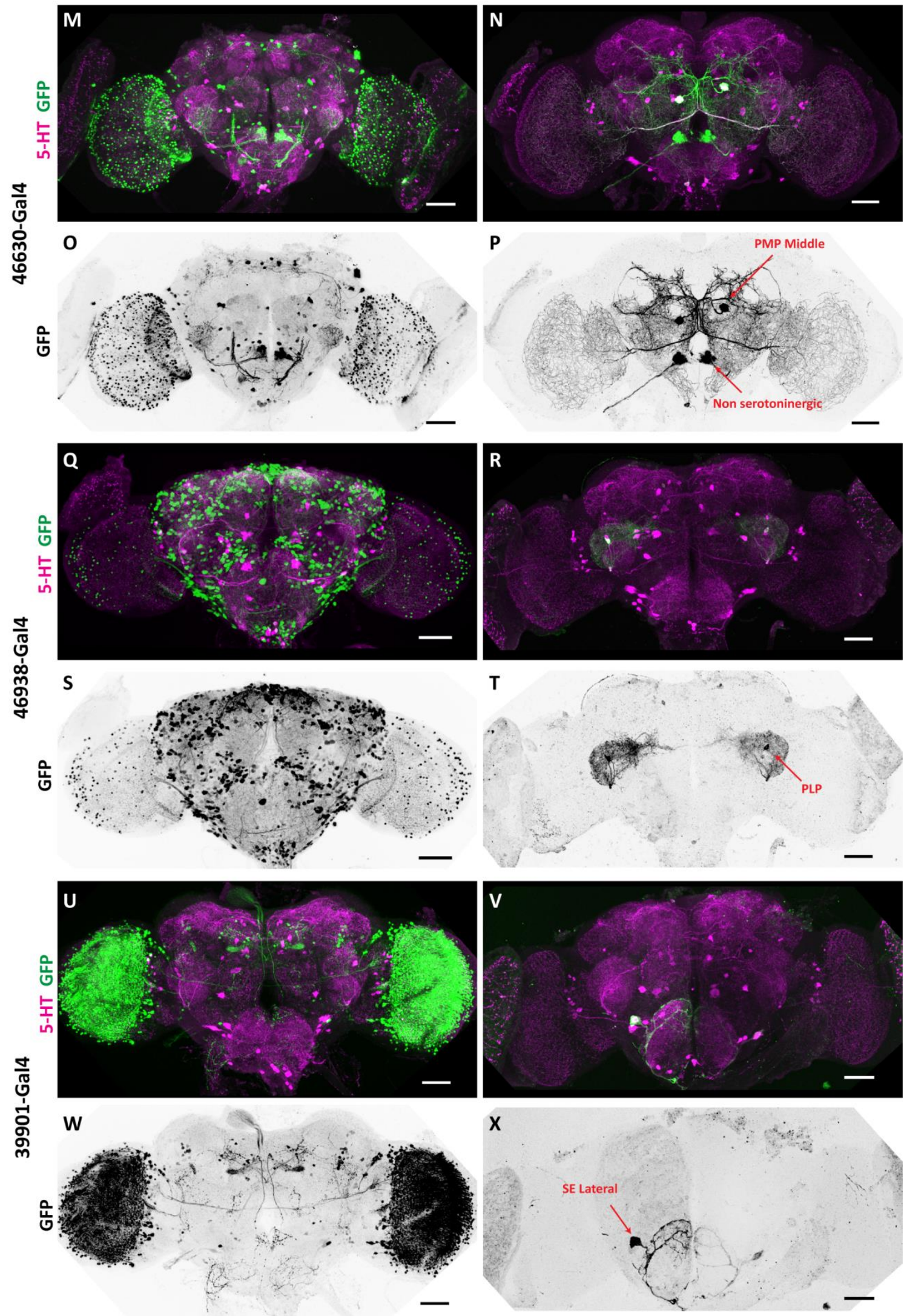


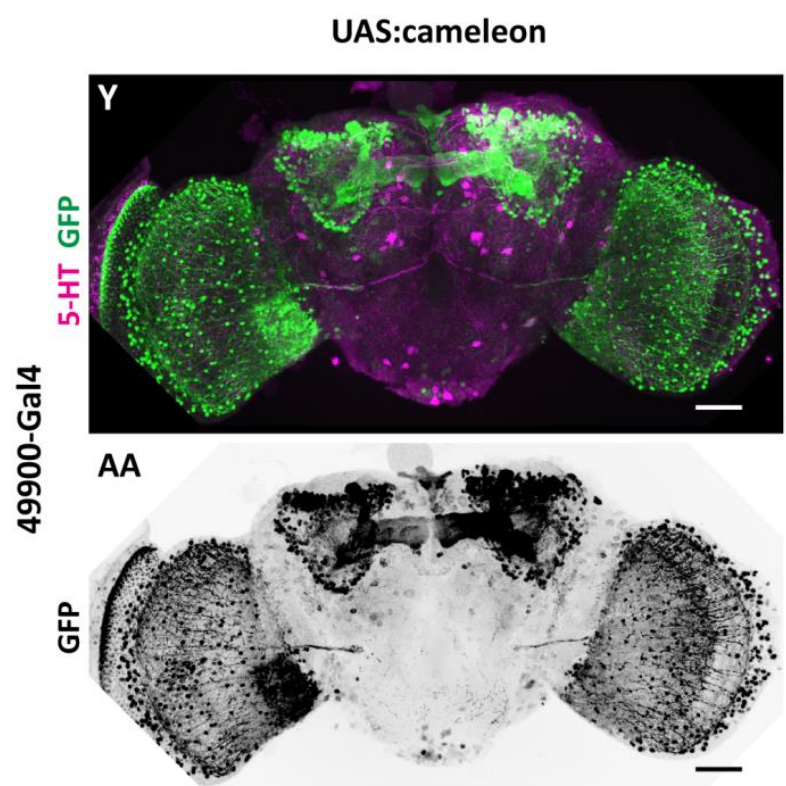

TRH-FIF; UAS:FRT-stop-FRT-mCD8:GFP
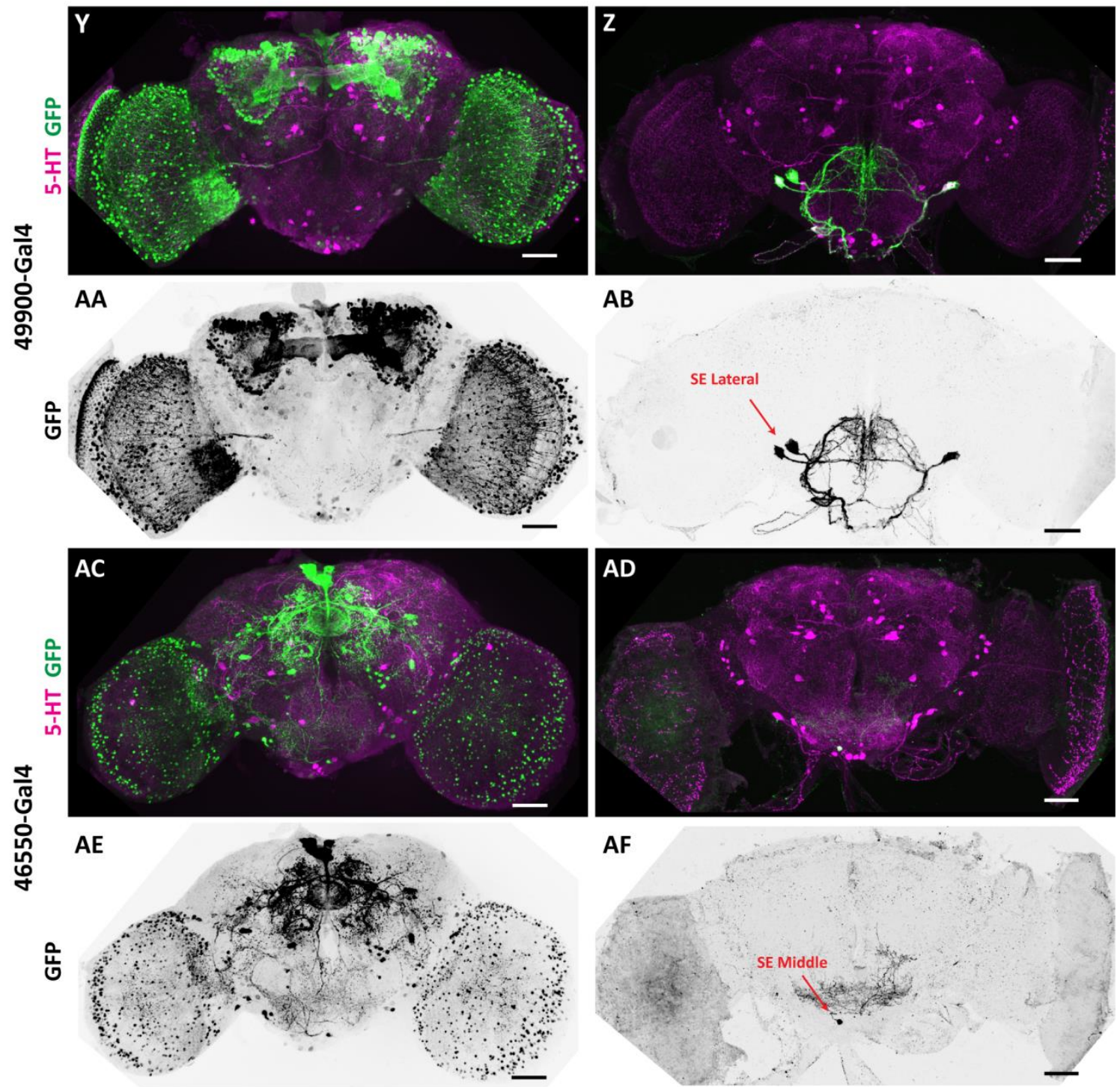

AB
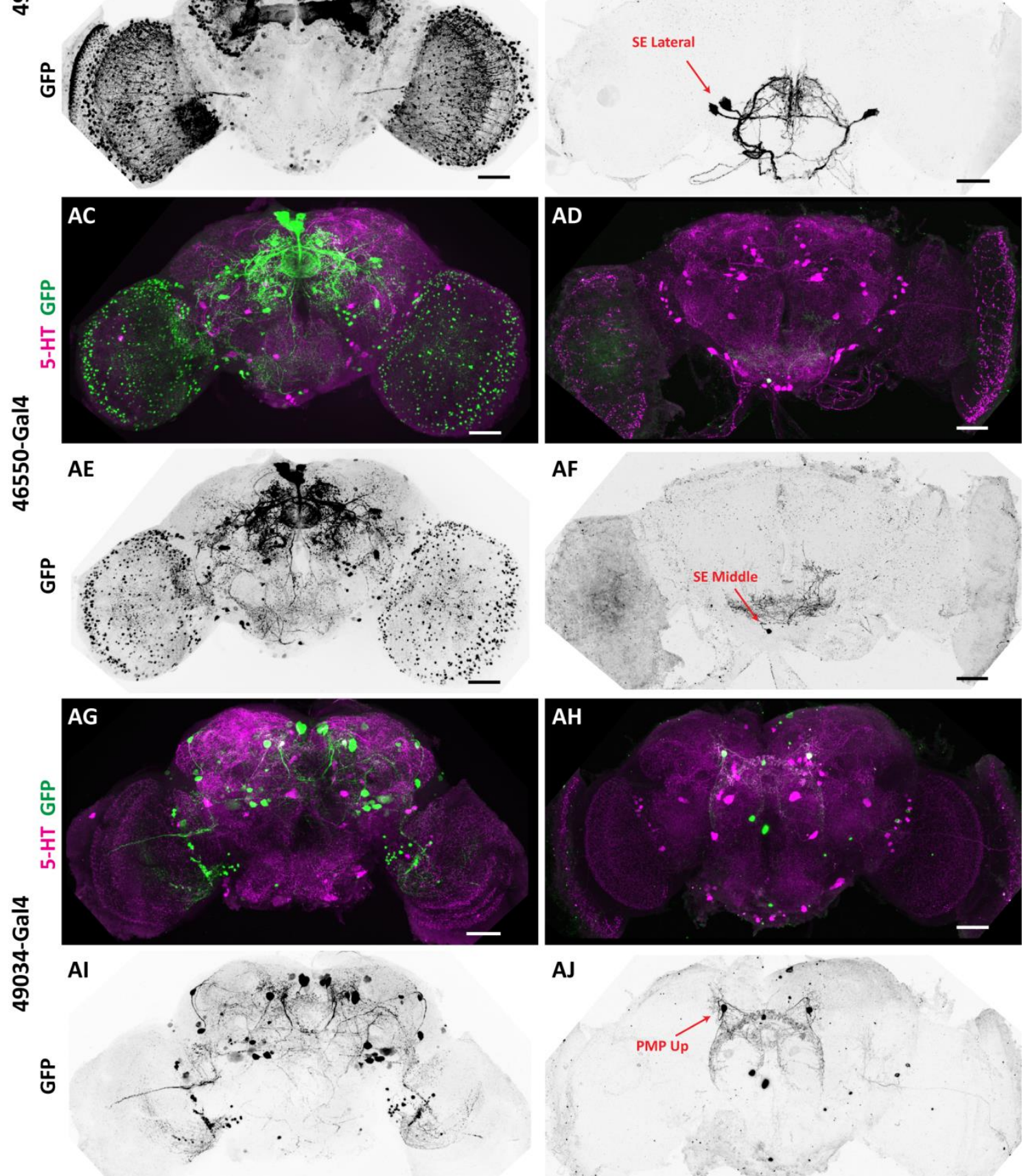

AF
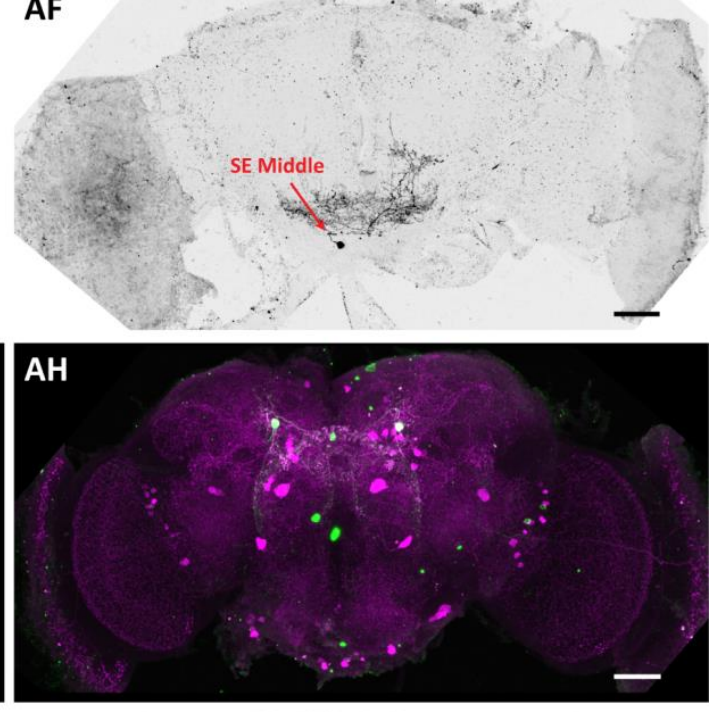

AJ

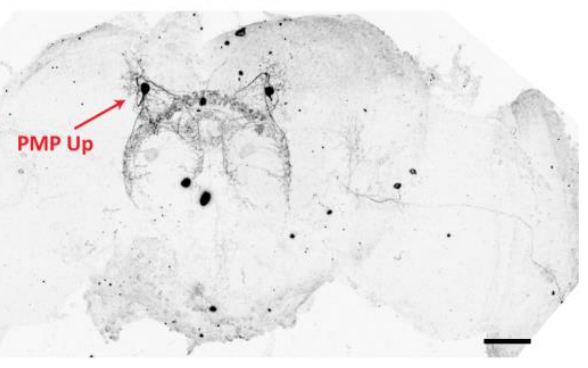




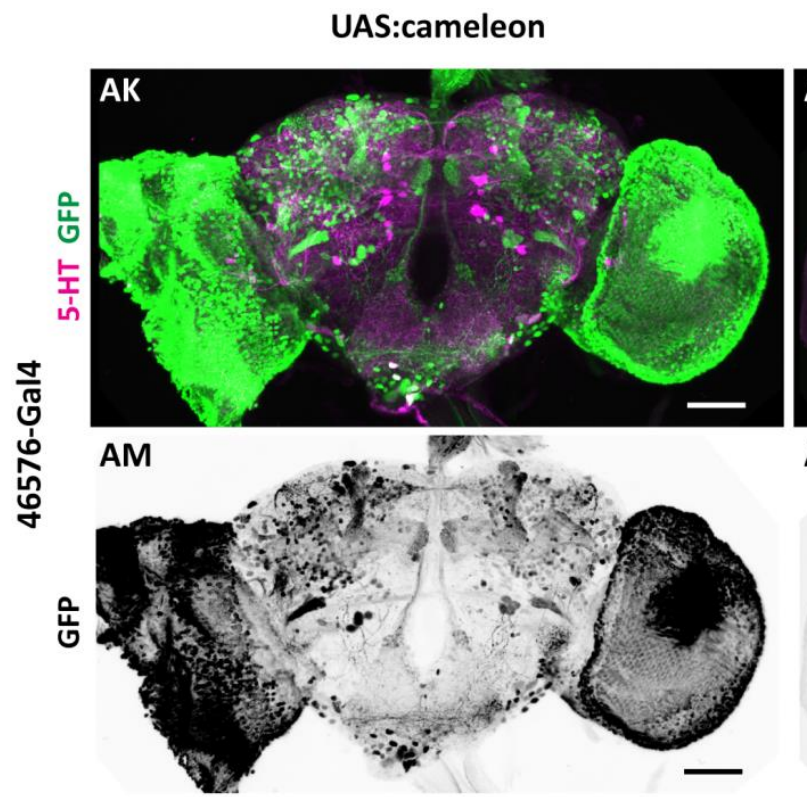

TRH-FIF; UAS:FRT-stop-FRT-mCD8:GFP
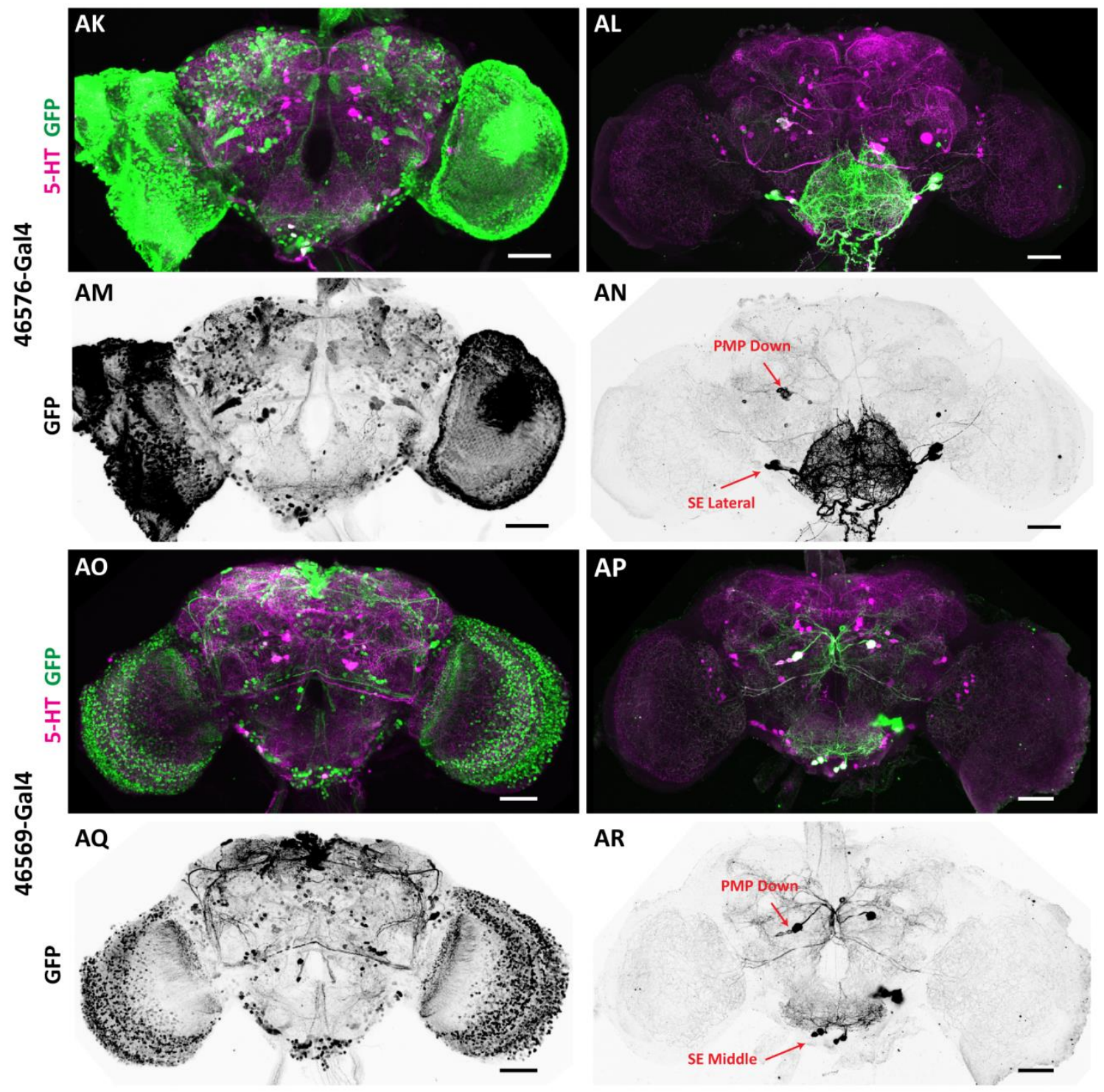

AR

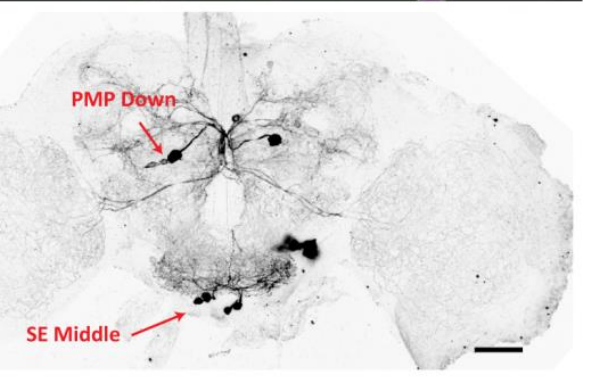

Figure 3.25. Visualizing and comparing the expression pattern of different Gal4 driver lines using the UAS-Gal4 system (images at the left) and intersectional approach (images at the right). The expression pattern of cameleon under the control of $11 \mathrm{Gal} 4$ driver lines was visualized by immunohistochemistry using anti-GFP and anti-5-HT antibodies. Using the intersectional approach, the expression of GFP was restricted to the serotoninergic neurons in w; TRH-FIF/+; UAS:FRT-stop-FRT-(2x)mCD8:GFP/ -Gal4 flies. For each Gal4 line the first row shows the merge images of GFP (green) and 5-HT (magenta) staining and the images in the second row show the GFP staining. The names of the serotoninergic clusters having GFP expressing cell(s) in the intersectional approach are indicated with red arrowheads. Scale bar: $50 \mu \mathrm{m}$ 
After generating the required functional tools for intersectional expression of the transgene, the next step was to monitor the arousal level of flies while thermoactivating subdivisions of the serotoninergic cells. For this purpose, two copies of the UAS:FRT-CD2-stop-FRT-mCherrydTRPA1 and TRH-FIF insertions in second and third chromosomes were recombined to generate w; UAS:FRT-CD2-stop-FRT-mCherry-dTRPA1, TRH-FIF/cyo; UAS:FRT-CD2-stop-FRT-mCherryITRPA1, TRH-FIF/TM3 line. This line was then crossed to 5 selected Gal4 lines to express mCherry-dTRPA1 transgene in subdivisions of serotoninergic neurons to identify the serotoninergic cells underlying the effect of serotonin on arousal state. The statistical analysis of stochastic study (section 3.7.1.2) pointed towards a role of cells within the PMP Down cluster. Among the screened Gal4 lines, no PMP down-specific Gal4 line was found. However, three Gal4 lines (46630-Gal4, 46576-Gal4 and 46569-Gal4) showed expression in the PMP down cluster and some other serotoninergic cells (including PMP Up and PLP neurons) and were therefore selected for further experiments (Figure $3.25 \mathrm{M}-\mathrm{P}$, Ak-AN, AO-AR). To exclude a potential involvement of PMP Up and PLP clusters in the modulation of arousal, behavioral performance obtained with this line was directly compared with the performance of two other Gal4 lines (46938-Gal4 and 49034-Gal4) that showed expression of the reporter in PMP up and PLP clusters, but not in the PMP down cluster (Figure 3.25 Q-T, AG-AJ). In order to verify the role of the serotoninergic cells of the PMP Down cluster on modulation of arousal, the progenies of the crosses (as the test groups) and the heterozygous parental lines (as the control groups) were tested in the walking velocity assay at $18^{\circ} \mathrm{C}$ and $32^{\circ} \mathrm{C}$. In the $18^{\circ} \mathrm{C}$ experiment, the test and control groups did not show any significant difference in the walking velocity (Figure $3.26 \mathrm{~A})$. However, the activation of serotoninergic neurons at $32^{\circ} \mathrm{C}$ caused a significant reduction in the walking velocity of the 46569>2x(UAS:FRT-CD2-stop-FRT-mCherrydTRPA1,TRH-FIF) and 46576>2x(UAS:FRT-CD2-stop-FRT-mCherry-dTRPA1,TRH-FIF) flies in comparison to the control groups. No significant difference between test and control groups for the three other Gal4 lines was observed (Figure 3.26 B). 
A

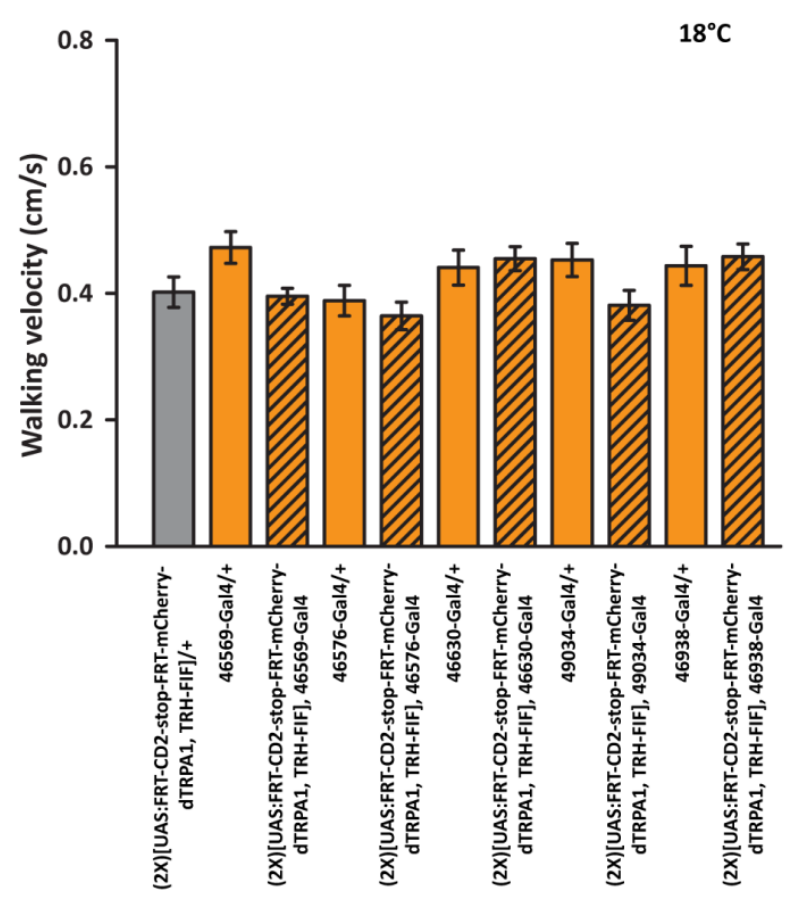

B

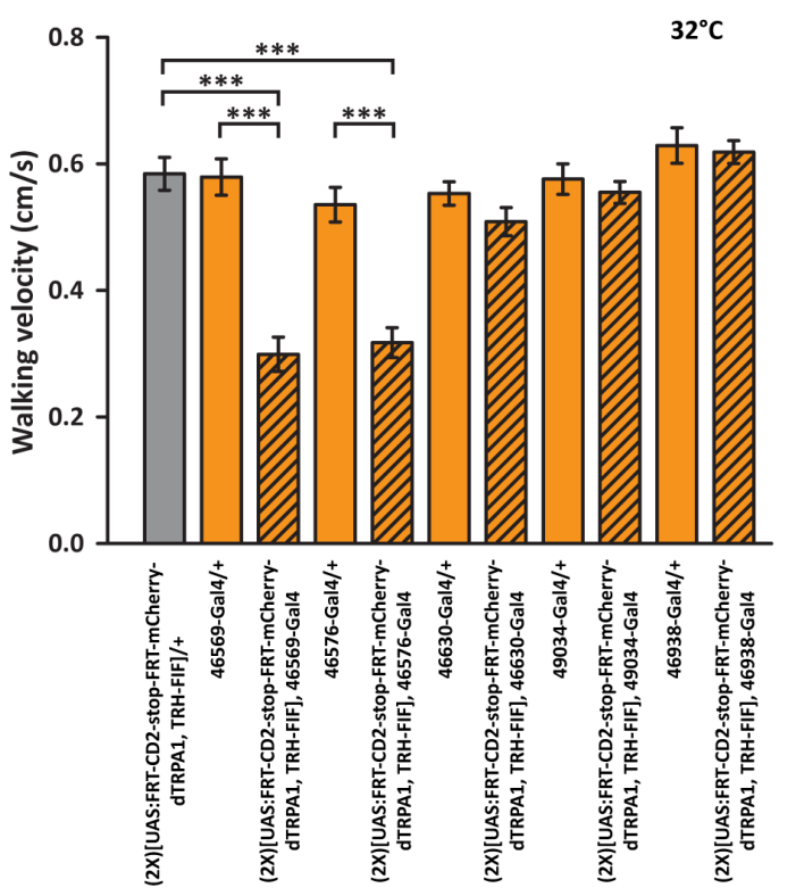

Figure 3.26. Thermoactivation of subsets of serotoninergic cells using an intersectional approach and its effect on walking velocity. The mChery-dTRPA1 was expressed in different subdivisions of serotoninergic neurons under the control of 5 Gal4 lines (46569-Gal4, 46576-Gal4, 46630-Gal4, 49034-Gal4 and 46938-Gal4). Gal4>2x(UAS:FRTCD2-stop-FRT-mCherry-dTRPA1,TRH-FIF) flies as the test group and the heterozygous parental lines as the control groups were tested in the walking velocity assay at $18^{\circ} \mathrm{C}$ and $32^{\circ} \mathrm{C}$. (A) The test and control groups did not show any significant difference in the walking velocity at $18^{\circ} \mathrm{C}$. (B) Activation of serotoninergic neurons at $32^{\circ} \mathrm{C}$ caused significant reduction in the walking velocity of the 46569>2x (UAS:FRT-CD2-stop-FRT-mCherry-dTRPA1,TRH-FIF) and 46576>2x (UAS:FRT-CD2-stop-FRT-mCherry-dTRPA1,TRH-FIF) flies in comparison to the control groups. However, 46630>2x(UAS:FRT-CD2-stop-FRT-mCherry-dTRPA1,TRH-FIF), 49034>2x(UAS:FRT-CD2-stop-FRT-mCherrydTRPA1,TRH-FIF) and 46938>2x(UAS:FRT-CD2-stop-FRT-mCherry-dTRPA1,TRH-FIF) flies did not show any significant difference in the walking velocity in comparison to the parental controls. One-Way ANOVA with post hoc Bonferroni correction. $\left({ }^{* * *}\right)$ : $\mathrm{P} \leq 0.001 . \mathrm{n}=29-32$.

The expression of mCherry-dTRPA1 in three cells in each brain hemisphere (one big and two small cells) within the PMP Down cluster was covered using 46569-Gal4 and 46576-Gal4 lines (Figure 3.27 B, D). In addition to the PMP Down cluster, 46569>2x(UAS:FRT-CD2-stop-FRTmCherry-dTRPA1,TRH-FIF) flies expressed mCherry-dTRPA1 in few cells of SE Middle and SE Lateral clusters (Figure 3.27 A) and one cell of PMP Up cluster in each brain hemisphere (Figure 3.27 B) and 46576>2x(UAS:FRT-CD2-stop-FRT-mCherry-dTRPA1,TRH-FIF) flies expressed mCherry-dTRPA1 in SE Middle and SE Lateral clusters (Figure 3.27 C, D), one cell of PLP cluster 
and one cell of PMP Up cluster in each brain hemisphere (Figure $3.27 \mathrm{D}$ ). Since the expression of mCherry-dTRPA1 under the control of 46569-Gal4 and 46576-Gal4 led to a reduction in the walking velocity, the activated cells in these two lines were interpreted as being involved in modulation of arousal. The effect of the big cell of PMP down cluster (Figure $3.24 \mathrm{~F}$ ), the cell of PLP clusters (Figure 3.27. J) and the cell of PMP UP cluster (Figure $3.27 \mathrm{H}$ ) on altering the velocity of the flies was excluded using 46630-Gal4, 46938-Gal4 and 49034-Gal4 lines, respectively (Figure 3.26).

SE Middle and SE Lateral clusters were less likely to be the responsible for the observed reduction in walking velocity. The expression pattern of mCherry-dTRPA1 in SE Middle and SE Lateral clusters in 46569-Gal4 and 46576-Gal4 did not show a complete overlap and the 46576Gal4 line covered more cells in these clusters than the 46569-Gal4 line. In addition, the stochastic study did not show any significant difference in walking velocity due to the activation of the cells in SE Middle and SE Lateral clusters in (Figure 3.20 E, F). In both 46569-Gal4 and 46576-Gal4 lines, which showed the reduction in the walking velocity, the mCherry-dTRPA1 was expressed in the two small cells in PMP Down cluster (Figure 3.27 B, D).

Taken together, the results of both, the stochastic and the intersectional study of serotoninergic neurons, indicate that the modulatory effect of serotonin on arousal level is mediated by a specific serotoninergic cluster and it is not a random effect of serotonin release. This study proposes a subdivision of cells within the PMP down cluster as a modulator of the arousal level in Drosophila. 
3. Results

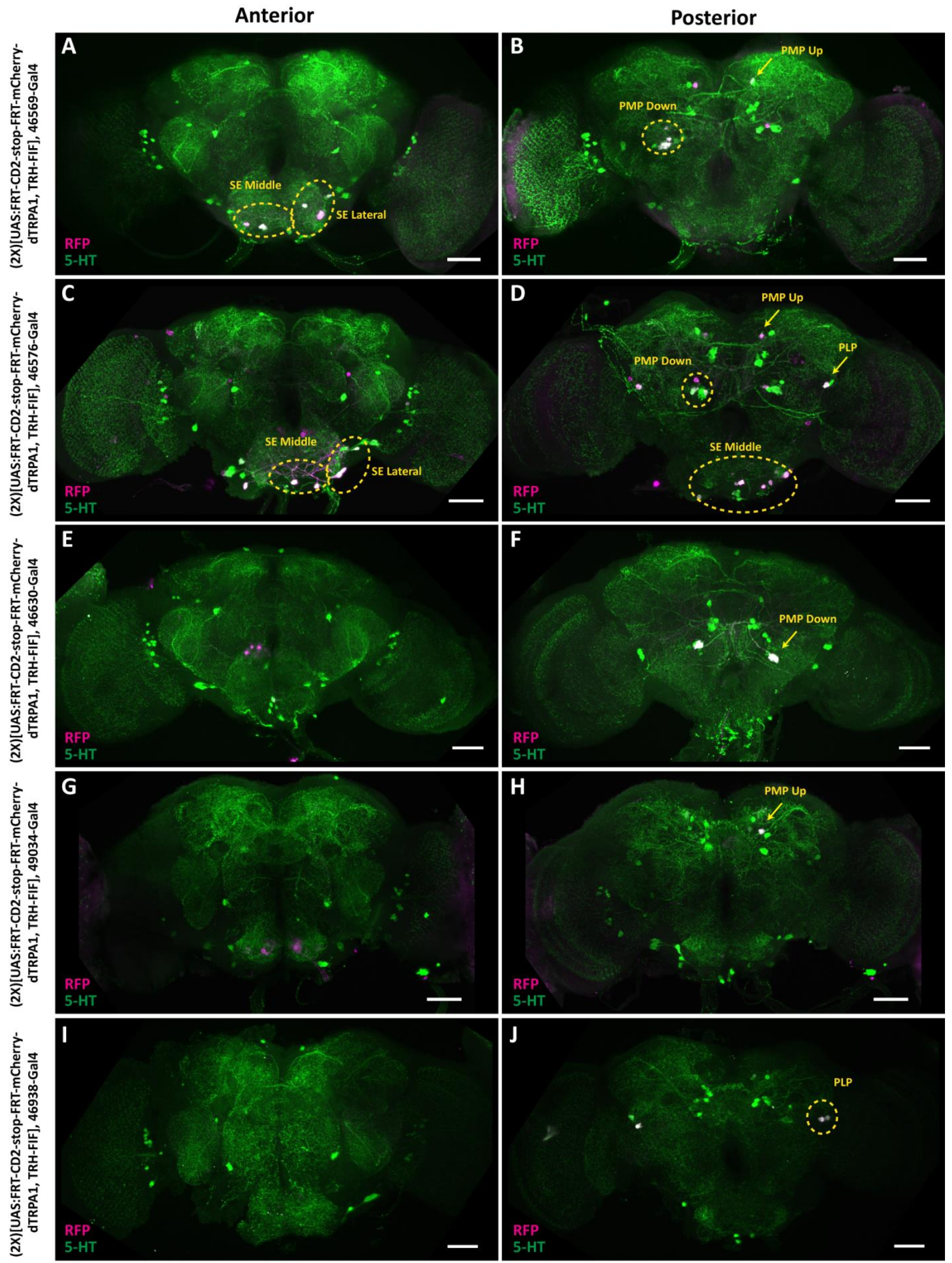


Figure 3.27. Visualizing the expression pattern of different Gal4 driver lines using an intersectional approach in Gal4>2x(UAS:FRT-CD2-stop-FRT-mCherry-dTRPA1,TRH-FIF) flies. The anterior (left) and posterior (right) views of the Drosophila brain expressed mCherry-dTRPA1 under the control of 46569-Gal4, 46576-Gal4, 46630-Gal4, 49034-Gal4 and 46938-Gal4 driver lines in the FLP positive cells. The expression pattern was visualized using an anti-RFP antibody against mCherry protein (magenta) and an anti-5-HT antibody (green). The staining in white color indicates the serotoninergic cells expressing mCherry-dTRPA1 which are marked with yellow circles and arrowheads. Scale bar: $50 \mu \mathrm{m}$ 


\section{Discussion}

Arousal is defined as an internal state of animals that regulates the behavioral performance. The optimized arousal state in animal is essential for motivating or inhibiting a specific behavior. It does so by regulating the response of an animal to external stimuli according to the internal physiological state. Based on different theories, arousal can be uni-dimentional and regarded as a concept that explains how every behavior is affected by a general, intrinsic state, or it can be multiple and task-specific (Hebb, 1955; Robbins, 1997; reviewed by Berlucchi, 1997; reviewed by Jing et al. 2009). Also in another model of arousal, the combination of general and task-specific kinds of arousal for regulating diverse behavior in animal is proposed (Pfaff et al., 2008). According to Pfaff, (2005a), arousal in animal is defined by three criteria: alertness to sensory stimuli, motor activity and emotional reactivity.

In a further sub-categorization of arousal, two forms of arousal consisting of endogenous and exogenous arousal exist. The sleep and wake states are the extreme ends of endogenous arousal and exogenous arousal can be described as the responsiveness to the external stimuli (Van Swindern and Andretic, 2003; Lebestky et al., 2009).

In the regulation of the arousal level as a fundamental factor for facilitating the initiation and termination of variety of the behaviors and, therefore, as a prerequisite for optimal performance in animals, a wide range of genes, hormones peptides and biogenic amines contribute (Pfaff, 2005b; Hildebrandt et al., 2010; Smith et al., 2014; Asahina et al., 2014). This redundancy in modulating mechanisms of arousal might be essential for its conservation when one system is damaged (Hildebrandt et al., 2010).

The modulatory effect of serotonin on the arousal state in the context of distinct behaviors was shown in different species. Additionally, serotonin is considered as a general inhibitor of behavioral responses (Depue and Spoont, 1986; Spoont, 1992) and it has been shown that this the neurotransmitter has an inhibitory action in the brain (Daw et al., 2002; Yan, 2002).

Studies on mammals demonstrate the a diminished serotoninergic neurotransmission is associated with increased aggression and anxiety disorders, obsessive compulsive disorder increased sexual arousal and over eating (Haller 2013; Hansen and Ross, 1983; Spoont, 1992; Hildebrandt et al., 2010). However, different serotonin receptors which are located both pre- 
and post-synaptically (Guo and Rainnie, 2010; review by Fox and Lowry, 2013) form a very complex system in the brain. Therefore, the effect of serotonin on regulation of distinct neuronal circuits is likely not simple and straight-forward.

In Drosophila, the modulatory role of dopamine in regulation of different kinds of arousal was shown in several studies. Dopamine regulates endogenous and exogenous arousal through different neuronal circuits (Andretic et al., 2005; Kume et al., 2005; Lebestky et al., 2009; Ueno et al., 2012; Liu et al., 2012; Kayser et al., 2014). However, the role of serotonin in modulating arousal was shown mostly in sleep and wake states (Yuan et al., 2006) and the neuromodulatory effect of serotonin on different forms of arousal is still unknown. Although in different behavioral contexts like courtship, aggression, locomotion, feeding, learning and memory, a regulatory effect of serotonin and its receptors was concluded (Neckameyer et al. 2007; Sitaraman et al., 2008; Alekseyenko et al., 2010; Becnel et al., 2011; Gasque et al., 2013; Silva et al., 2014), altering the serotonin level in the brain did not abolish or induce any of these behaviors. Therefore, the influence of serotonin in the regulation of behavior is unlikely to be causal. There is a possibility that the regulatory effect of serotonin on variety of behaviors can be indirect and it may originate from modulation of a general arousal level in flies.

\subsection{A thermogenetic approach to dissect serotonin- dependent arousal in Drosophila}

In this study the potential modulatory effect of serotoninergic neurons on general arousal and also exogenous generated arousal was explored. A particular focus of this study was the question of whether serotonin acts "as a system", i.e., behavior would be influenced by the amount of the neurotransmitter in the brain regardless of the neurons that release it, or whether any regulating role can be assigned to specific circuits, i.e., to specific populations of neurons that release serotonin.

In order to determine whether a specific population of the neurons is sufficient or required for a specific behavior, different activators and silencers can be expressed ectopically in neurons and the effect in the level of behavior can be studied. In this study we used a thermogenetic approach to activate the neuronal activity or silence synaptic transmission using the dTRPA1 channel (Hamada et al., 2008) and the temperature-sensitive shibire protein (Kitamoto, 2001), 
respectively. The expression of these proteins in serotoninergic neurons enabled us to remotely activate or block the neurons by changing the temperature. The disadvantage of the thermogenetic approach for manipulation of the neurons is that the behavioral experiment cannot perform at a "normal" physiological temperature range. In order to overcome this problem and exclude the effect of temperature on the behavioral performance, the behavior of control flies at higher temperatures is considered as the baseline. The difference in performance of the test flies in comparison to this baseline is regarded to result from the neuronal manipulation. Another limitation for thermoactivation of the neurons is that dTRPA1 is not the appropriate tool for continuous activation. Therefore, the utility of this technique is limited to the short behavioral experiments and ITRPA1 channel is not effective for depolarization of neurons after long continuous activation (Figure 3.12; Inagaki et al., 2014). Some neurons due to the continuous activation develop a depolarization block (Bianchi et al., 2012). Nevertheless, optogenetic activation of the neurons using new version of red-shifted channelrhodopsin might be a substitution for dTRPA1 in future experiments (Inagaki et al., 2014).

\subsection{Serotoninergic neurons: a modulator of the general arousal level in Drosophila}

For measuring the arousal level in Drosophila, the locomotor activity is the common indicator. Hyperactivity of the flies is directly linked to a higher arousal level (Shaw et al., 2000, Andretic et al., 2005), and, as mentioned before, one general criterion that defines arousal (Pfaff. 2005a). In our experiment, thermoactivation of serotoninergic neurons through the dTRPA1 channel led to a significant reduction in walking velocity (Figure 3.4). However, since several studies indicate a regulatory effect of serotonin on the motor behavior, it was not possible to conclude the low arousal level from the observed activity reduction. It has been shown that acutely diminishing neuronal serotonin in TRH-null mutant causes significantly reduced locomotor behavior in adult flies (Neckameyer et al., 2007). Conversely, another study shows that, elevation of serotonin level using fluoxetine and MDMA as the serotonin transporter inhibitor drugs reduces the locomotion in Drosophila larvae (Silva et al., 2014). This controversy seen in the studies, in which the role of serotonin function and locomotion is investigated, is maybe due to the methodological differences between the studies. For instance, it is important 
to consider that the effect of serotonin on modulation of distinct behaviors can be different in constitutive or temporal manipulation of the serotonin level. Since serotonin counts as a critical factor in development of synaptic connectivity (Gaspar et al. 2003), a locomotor deficit observed in Neckameyer et al.(2007) study can be the result of developmental defect which was caused by acute reduction in serotonin level in the embryonic stage. Furthermore, Neckameyer et al. (2010) demonstrated that the acute reduction in the serotonin level in the developmental stage leads to increased branching of the serotoninergic fibers and increased size and number of varicosities along the arborizations projecting to the gut (Neckameyer, 2010).

Taken together, when a locomotion effect is used as an index for the arousal state, it is crucial to distinguish between a motor deficit and a lower motivational level. For this purpose, the behavioral consequence of activated serotoninergic neurons and activated motor neurons were compared in several startle-inducing paradigms. The depolarization of motor neurons through activation of dTRPA1 channel results in paralysis of the flies. Therefore, it was regarded as the suitable control, which was the depiction of motor deficit in flies. Our results indicated that the stimulus reactivity of the flies with activated serotoninergic neurons to the mechanical stimulation was unaffected and manipulation of these neurons did not lead to locomotor impairment (section 3.3.3). In other words, the flies could move, they just would not do so. Hence, we conclude from our experiments that the observed reduction in walking activity of the flies was in fact the representation of a low general arousal level, in the way that conceivably the serotoninergic neurons modulate distinct behaviors in flies by modifying the motivational status.

In addition to conclusions about walking capability, the startle-response experiments could address the modulatory role of serotonin in responsiveness to a mechanical external stimulus as the exogenous generated arousal. We have shown that, although the activation of serotoninergic neurons modulates the general arousal level in flies, startle-induced reflexes of the flies are still functional. Though our experiments were designed in a way to examine the possible inhibitory effect of serotoninergic neurons on the responsiveness of the flies, the possible positive regulatory effect could not be examined. For investigating this possibility the quantitative behavior assay for mechanical startle-induced, which was described by Lebestky et al. (2009), can be used in the future studies. 
Based on the arousal model in gastropod molluscs, serotoninergic neurons act as general arousal elements, which may perform along with more specialized arousal elements.

To investigate the possible similarity for the function of serotoninergic neurons in Drosophila, the effect of activating serotoninergic neurons on responding to hunger as the internal stimulus in the presence of food was explored. As mentioned earlier, alertness to sensory stimuli is another general criterion for arousal (Pfaff, 2005a). Our results indicate that flies with activated serotoninergic neurons, although having hunger as the internal force, do not show the motivation for eating (section 3.3.4). This evidence is another confirmation for a modulatory role of serotoninergic neurons in general arousal level in Drosophila. Our results are also in accordance with studies that indicate an inhibitory effect of serotonin on eating behaviors in mammals (Brewerton, 1995). In the pathology of bulimia nervosa disease, which is defined by lack of control over eating, dysregulation of the serotonergic system is one of the major neurobiological causes (Steiger et al., 2004). For example, dysregulation of 5-HT1A as the inhibitory receptor (Barnes and Sharp, 1999) in the hypothalamus may diminish the eating specific system's negative feedback loop making it difficult to terminate eating (Hildebrandt et al., 2010). The results of the study of Neckameyer et al. (2010), which showed that the increased feeding rate in Drosophila larvae was the consequence of reduction in the serotonin level using inducible TRH RNAi (Neckameyer, 2010), could also be explained by a modulatory effect of serotoninergic neurons on general arousal.

\subsection{Conclusions and limitations using pharmacological approaches}

PCPA as an inhibitor of the rate limiting enzyme tryptophan hydroxylase in the serotonin biosynthesis pathway has been used as a serotonin depletor in many organisms like mouse, rat, cat and dog (Jha et al., 2006; Haga et al., 1996; Blundell, 1984). Moreover, in Drosophila the effect of PCPA was investigated in the context of distinct behavior like locomotion, flight and learning (Kamyshev et al., 1983; Banerjee et al., 2004; Vaysse et al., 1988; Dasari et al., 2007). However, there are some contradictory results about the efficacy of PCPA in the Drosophila brain. Previous reports suggested that systemic administration of PCPA does not inhibit serotonin synthesis in the fly brain (Coleman and Neckameyer, 2005). The results of our 
experiment indicate that PCPA administered with food led to a partial reduction of serotonin level in Drosophila brain. To investigate whether the neurotransmitter serotonin is indeed a modulator of general arousal in Drosophila, serotoninergic neurons in which the serotonin level was partially depleted were thermoactivated. Interestingly, activation of serotoninergic neurons led to a significantly higher arousal level in the flies with depletion of serotonin in comparison to the intact flies (Figure 3.14). The pharmacological approach, therefore, suggests that it is serotonin, not any potential co-transmitter of the activated neurons, that modulates the arousal states in the flies.

\subsection{Sufficiency and necessity of serotonergic neurons to modulate the arousal level}

As mentioned above, another criterion to ask for is the necessity of neurons in modulating the behavior. The further exploration on the modulatory role of the serotoninergic neurons in the arousal level indicates that blocking the neurotransmission from serotoninergic neurons using temperature-sensitive shibire (Kitamoto, 2001) led to increases in the walking activity level. In other words, silencing serotonergic neurons was shown to be sufficient to release an enhanced arousal level. However, there are some controversies about the effect of serotonin depletion on the behavior of animals. For instance, in consistence with our results, Kamyshev et al. (1983) showed that a reduction in the serotonin level leads to the elevated locomotor activity. Conversely, according to Banerjee et al. (2004), depletion of serotonin causes reduction in flight ability in the flies. Likewise, in mammals reduced serotonin function is reported to have opposite behavioral effect based on the different studies (Blundell, 1984; Spoont, 1992; Daprada et al., 1985; Hansen and Ross, 1983; Myoga et al., 1995). Perhaps the base of observed controversies in these experiments is due to the methodology. For example using different concentration of drugs, using different paradigms for behavioral tests or targeting different regions of the brain in the case of mammals can generate different results.

Taken together, our finding suggests that the regulatory effect of the serotoninergic neurons on arousal state is bidirectional, in a way that activation of these cells decreases the arousal level while blocking them causes a higher arousal level in the flies. 


\subsection{Serotoninergic neurons: modulator of sleep/activity states}

Sleep is a general occurrence that exists in every species from mammals to insects (reviewed by Allada and Siegel, 2008). In Drosophila the constant periods of immobility during the night is regarded as a sleeplike state which demonstrates all the fundamental features of mammalian sleep (Hendricks et al., 2000; Shaw et al., 2000). The sleep behavior in flies as well as the circadian periods of immobility shows other sleep related criteria such as a decreased arousal threshold which was measured using vibratory, visual, or auditory stimuli (Shaw et al., 2000; Nitz et al., 2002; Huber et al., 2004). Moreover, sleep in Drosophila is similar to the mammals in that it is homeostatically regulated (Borbély and Achermann, 1999), which means that sleep deprivation leads to a sleep rebound described by an increase in the duration and/or in the intensity of sleep (Huber et al., 2004, reviewed by Cirelli and Bushey, 2008). Previous studies in Drosophila implicate the involvement of biogenic amines in regulation of sleep-wake signaling (Andretic et al., 2005; Kume et al., 2005; Crocker and Sehgal, 2008; Yuan et al., 2006). Dopamine is regarded as one of the essential biogenic amines involved in regulation of endogenous arousal in the flies (Andretic et al., 2005; Kume et al., 2005; Van Swinderen and Andretic, 2011; Kayser et al., 2014). The elimination of the dopamine transporter (DAT) activity in fumin mutant flies generates a sleepless phenotype (Kume et al., 2005). Moreover, administration of methamphetamine, which increases the dopamine level by blocking the dopamine transporter, leads to short sleep and increased wakefulness in the flies (Andretic et al., 2005). The thermoactivation of entire or specific subsets of dopaminergic neurons increases the arousal level by reducing the sleep in the flies (Ueno et al., 2012; Liu et al., 2012; Kayser et al., 2014).

In this study, in order to investigate the modulatory effect of serotoninergic neurons on endogenous arousal, we used temperature sensitive shibire to block the serotoninergic neurons (Kitamoto, 2001). Our results indicate that blocking the signal transduction in serotoninergic system leads to short night sleep in the flies. This finding is consistence with previous report which showed sleep promoting role of serotonin in Drosophila (Yuan et al., 2006). According to the report of Yuan et al., an elevated serotonin level induced by the serotonin synthesis precursor (5HTP) leads to increase sleep. In addition, they showed that the serotonin receptor 5-HT1A expressed in the mushroom bodies has a role in sleep regulation. 
Overall, we conclude from these evidences that serotoninergic signaling modulates sleep-wake states in the flies. Noteworthy, the reverse effect of dopamine on regulating sleep is an important indicator of a complementary function of these two neuromodulators in Drosophila.

\subsection{Dissecting the neuronal circuits underlying modulation of arousal}

In order to dissect the neuronal circuits underlying a certain behavior, different genetic strategies for manipulating individual neurons or subsets of neurons are commonly used in Drosophila research (Pfeiffer et al., 2010; reviewed by Venken et al., 2011).

Generating a mutant library for different genes using transposon mutagenesis (St Johnston, 2002; Schuldiner et al., 2008) and screening them in the specific behavioral assay is one approach. For example, Dubnau et al. (2003), using a P element transposon as a mutator, generated 6681 mutants and screened them for defective memory to identify the memory genes.

Screening thousands of Gal4 lines to manipulate the neuronal activity in the level of behavior to identify the neurons required for specific behavior is another approach (Flood et al., 2013; Vogelstein et al., 2014). For example, in the study of Gordon and Scott (2009), 311 Gal4 lines were used for a screen in a proboscis extension reflex (PER) paradigm. In order to identify the required neurons for taste behavior, they performed a mosaic analysis of the selected lines using a stochastic approach.

Also the MARCM technique (Mosaic analysis with a repressible cell marker), which generates uniquely labeled single-cell clones in the Drosophila central nervous system, is another way to investigate the neuronal circuits in the context of anatomy or functionality (Marin et al., 2002; Wu et al., 2013).

For restricting transgene expression in order to understand how individual neurons, or subsets of neurons, contribute to the function of neuronal networks, an increased number of methods are developing. However, each of these methods has benefits and also limitations. 
In this study we designed a two-step approach to investigate the neuronal circuit underlying regulation of arousal state in Drosophila. In the first step using a stochastic approach, the effect of activation of different subset of neuronal population on modulating arousal level was analyzed. In this way, we were able to define a subdivision of the cells as a candidate and rule out the effect of many other subdivisions. In the next step, using the intersectional approach by combining the UAS-Gal4 and FLP-FRT systems, we restricted transgene expression in the candidate subset of neurons and so as to verify the responsible neurons modulating the arousal level in flies we focused on manipulating the activity of that subset.

In our strategy, the stochastic approach was the substitution of the behavioral and anatomical screening of Gal4 lines covering all subsets of serotoninergic neurons. Since the Drosophila brain consist of $91 \pm 13$ (mean \pm SD) serotoninergic neurons (Figure 3.1), an enormous number of Gal4 lines which cover distinct subset of neuronal population would have been required for manipulating neuronal activity of the different subtypes of the serotoninergic neurons. In addition, expression patterns of Gal4 lines are usually not restricted to one type of neurons and the effect of other cell population on behavior cannot be excluded. Thereby, the benefit of our two step strategy was that in the first step the contribution of many subsets of cells was excluded by arbitrary activation of few serotoninergic neurons. For the specific activation of distinct subsets in the second step only a limited number of Gal4 lines were required. Additionally, another advantage of the stochastic approach was the option to manipulate diverse number of the cells in many different combinations. In order to achieve this opportunity using other techniques like Gal4 collections, even more number of lines should be screened to cover different cell combinations.

However, our technique had also limitations and because of the arbitrary fashion, mapping the activity pattern was required to be defined in many individual animals. Overall, it has to be considered that these techniques for restricted transgene expression including our strategy are productive but effortful. 


\subsection{Identification of specific serotoninergic neurons underlying the modulation of arousal state in Drosophila}

We demonstrated that serotoninergic neurons regulate arousal in flies. However, it was important to investigate whether the modulatory effect of serotonin on arousal level is a general effect and whether it can be elicited from any serotoninergic neurons or whether the regulatory effect of serotonin on arousal is evoked by certain and specific subtype of serotoninergic neurons.

In this study, using arbitrary thermoactivation of distinct subsets of serotoninergic neurons, the potential correlations between number and/or subtypes of activated serotonergic neurons and the arousal phenotype was verified (Figure 3.18; Figure 3.20). The result of our stochastical experiment reveals that a specific subset of serotoninergic neurons, located in the PMP Down cluster, is the best candidate subdivision which contributes to a modulation of arousal level in flies (Figure 3.21).

In order to examine the sufficiency of PMP Down cluster for decreasing the arousal level, using the intersectional approach, the effect of thermoactivation of this cluster was verified (Figure 3.22). Overall, we demonstrate that two cells in the PMP Down cluster are essential for the effects of serotoninergic neurons on arousal.

Although, activation of the entire serotoninergic system and activation of cells in PMP Down cluster both caused the significant reduction in the general arousal level in the flies, the quantity of the observed reduction was not the same. Therefore, we suggest that PMP Down cluster is the essential subset of serotoninergic neurons in modulation of arousal level, but from our experiment we cannot exclude a potential contribution of other subsets and it may well be that other serotoninergic neurons have a synergistic effect.

Overall, we could confirm that the regulatory effect of serotonin on arousal can be elicited from a specific subset of serotoninergic neurons and is, therefore, not a general effect of the entire serotoninergic "system". Additionally we showed that activation of subset of the cells in the PMP Down cluster is sufficient to induce a reduction in the general arousal level in the flies. However, we could not exclude the potentiate influence of other serotoninergic cells in this modulatory circuits. Therefore, to draw any conclusion about synergistic effect, a more comprehensive characterization of the combinatory effect of PMP down cluster with other 
subsets of serotoninergic neurons specifically the cells in LP and PMP Middle cluster is essential in future studies.

\subsection{Interaction of serotonin and other aminergic systems in the regulation of arousal}

Noteworthy, in mammals, several studies indicate anatomical and functional interactions between dopaminergic and serotoninergic systems (Hetey et al., 1985; Wong et al., 1995; Kapur and Remington, 1996; Sasaki-Adams and Kelley, 2001; Daw et al., 2002). It is proposed that the dopaminergic system is involved in the activation of motivated behavior and that the serotoninergic system plays a role in the inhibition of the behaviors (Spoont, 1992; Ikemoto and Panksepp, 1999; Daw et al., 2002; Yan, 2002). As an example, hyperactivity in dopaminergic system as well as hypoactivity in serotoninergic system has been associated with impulsive aggression (reviewed by Seo et al., 2008). Moreover, several lines of evidence indicate that these two systems have specifically a reciprocal interaction (Daw et al., 2002; Yan, 2002) and it has been shown that a balance between dopamine and serotonin activity is essential, e.g., in defense related behaviors (Deakin and Graeff, 1991).

Moreover, some evidences about complementary or redundant function of biogenic amines also exist in Drosophila (Chen et al., 2013). Moreover, during the developmental stage, the interaction between the dopaminergic system and the serotoninergic system is necessary for an appropriate generation of the neural feeding circuit (Neckameyer and Bhatt, 2012). According to our results, serotonin has an inhibitory influence on the arousal level in the flies. On the other hand, it has been shown that dopamine signaling promotes arousal especially through two dopaminergic clusters, PPL1 and PPM3, innervating the fan shape body (Andretic et al., 2005; Kume et al., 2005; Liu et al. 2012; Ueno et al., 2012; Kayser et al., 2014).

To summarize the findings of the present study and the available evidences from previous studies, a simple model for the modulatory mechanisms underlying arousal in Drosophila is proposed here (Figure 4.1). 


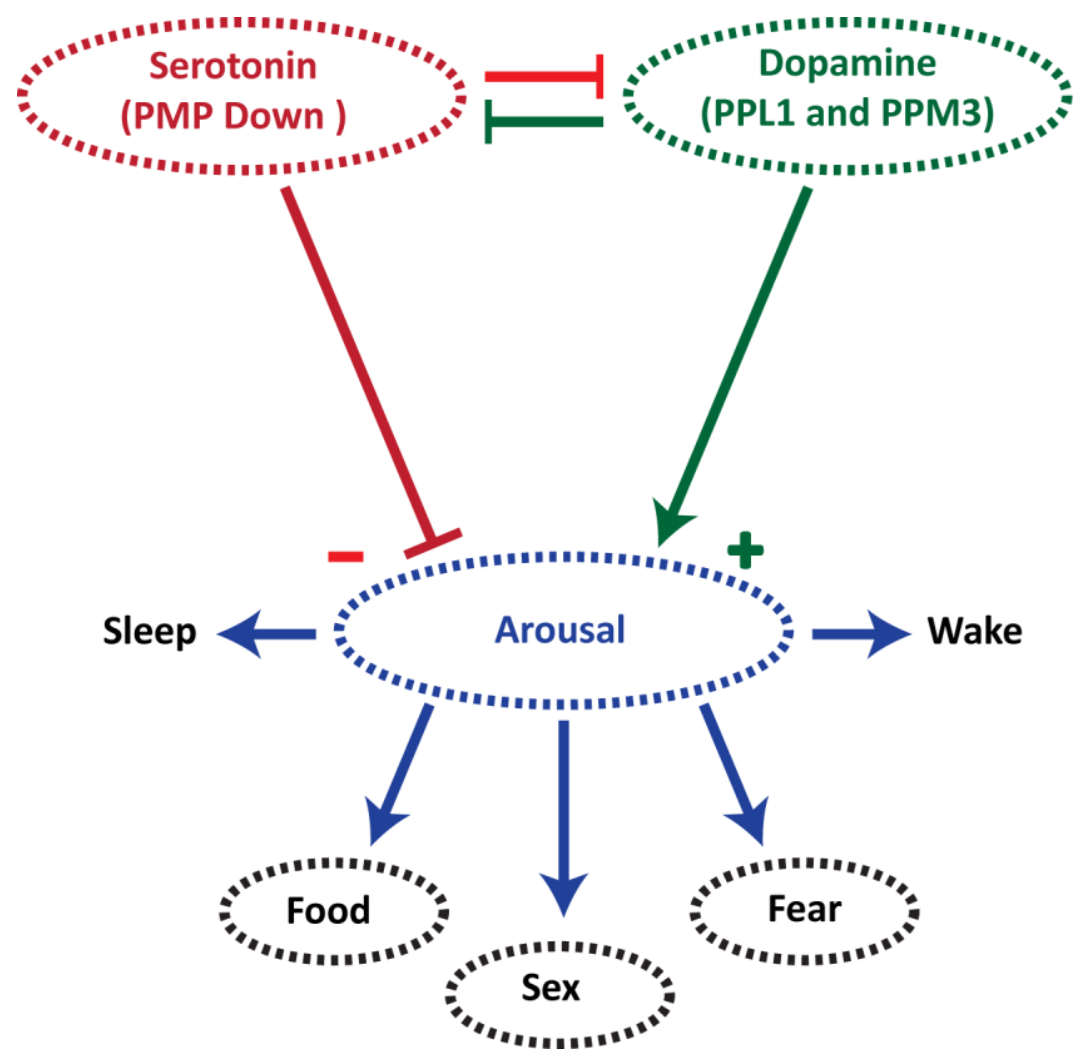

Figure 4.1. A working model describing a potential interaction of dopaminergic and serotoninergic systems in the modulation of arousal in Drosophila.

According to our proposed model, specific dopaminergic and serotoninergic circuits reciprocally interact with each other to adjust an optimal arousal level for proper behavioral performance in Drosophila. Since the two circuits have opposing effects on target regions in the brain, they necessarily need to inhibit each other. This way it is ensured that the circuit which is activated stronger suppresses the opposing circuit. We found that the serotonergic system not only influences locomotor activity and sleep (general arousal), but also the motivation of hungry flies to eat (task-specific arousal). Therefore, it is suggested that the aminergic circuits regulate a general endogenous arousal state, which in turn influences the diverse sub-aspects of biological relevant, task-specific arousal (i.e. the responsiveness to certain external stimuli). Our study proposes that the serotonergic PMP Down cluster is the essential element in this circuit for reducing arousal. However, the potential influence of other serotoninergic cells cannot be excluded.

Future studies are required to elucidate the brain regions which are innervated by the cells in PMP Down cluster and the neurons that provide input to the cells in the PMP Down cluster. It 
will be further of interest which serotonin receptors are involved in this pathway. Some future behavioral experiments are necessary to further determine the alertness to different stimuli, in particular in the context of sex and fear. This can be tested in courtship assays, for example by determining the responsiveness to courting. Fear and reflexive responses to mechanical stimuli can be distinguished in olfactory learning assays where an odor is associated with punishment. It needs to be confirmed that the serotoninergic circuit regulates central arousal rather than the peripheral response to the sensory stimuli. This can be tested using proboscis extension response of the flies to the food related stimuli. The ultimate goal is to describe the ascending and descending circuits which connect stimulus, neuronal activity and behavioral response. 


\section{Summary}

For animals, an optimal arousal state is essential for motivating or inhibiting a specific behavior. Arousal is regarded to be an internal factor that influences the initiation and execution of different behaviors, and is usually defined by three criteria: alertness to sensory stimuli, motor activity and emotional reactivity. A large amount of studies across many species have shown that different biogenic amines, like dopamine, serotonin or norepinephrine, contribute fundamentally to the neuronal substrate of arousal.

In the first part of this study the potential modulatory effect of serotoninergic neurons on arousal in Drosophila was explored. To do so, a new transgenic line was generated that allows for both the thermogenetic activation of the serotonergic neurons and their visualization (i.e., identification). Furthermore, a behavioral assay was established to use walking velocity of individual flies as a readout of the animals' arousal state. I could show that thermoactivation of serotoninergic neurons located in the brain region, but not in thoracic ganglia, led to a significant reduction in walking velocity. Furthermore, I could distinguish the behavioral consequence of activated serotoninergic neurons from those resulting from activated motor neurons by testing the startle-induced, reflexive response of the flies in several paradigms such as negative geotaxis, shock avoidance and flight. The reflexive stimulus reactivity of flies was unaffected by an activation of serotoninergic neurons, which means that the manipulation of these serotoninergic neurons did not lead to a locomotor deficit. In other words, the flies could move, they just would not do so.

I further investigated the alertness of the flies to sensory stimuli as the second criterion of arousal. Serotoninergic neurons of starved flies (hunger representing an internal motivational factor) were activated in the presence of food (as an external stimulus). My results show that activation of serotoninergic neurons prevents starved flies from feeding.

In addition, I investigated the modulatory effect of serotoninergic neurons on endogenous arousal (sleep-wake rhythms). I used temperature sensitive shibire expression to reversibly block serotoninergic neurons and found that this manipulation leads to shortened night sleep and increased activity during the night.

In the second part of the study I asked whether the neuronal mechanism underlying arousal is a global function of the neurotransmitter serotonin in the brain, or whether it is localized to the 
function of specific circuits. Therefore, I applied different genetic strategies to manipulate small subsets of neurons. In the first step, using a stochastic approach, the locomotor phenotype of flies, in which different subsets of serotonergic neurons were activated, was analyzed. In this way, I was able to identify specific subdivisions of serotoninergic cells, namely those of the PMP cluster, as a candidate circuitry that underlies the modulation of arousal. In the next step, using an intersectional gene expression approach, I restricted transgene expression to candidate subsets of neurons and could, thereby, verify that two individual, serotoninergic cells of the PMP cluster are sufficient to modulate the arousal level in flies.

Overall, I could show that serotoninergic neurons affect the arousal level of the fly, and that this modulatory effect can be elicited from a specific subset of serotoninergic neurons in PMP down cluster. In future studies, it will be essential to address potential combinatorial effects of the PMP down cluster with other subsets of serotoninergic neurons. 


\section{References}

Alekseyenko, O.V., Lee, C., and Kravitz, E.A. (2010). Targeted manipulation of serotonergic neurotransmission affects the escalation of aggression in adult male Drosophila melanogaster. PLoS ONE 5, e10806.

Allada, R., and Siegel, J.M. (2008). Unearthing the phylogenetic roots of sleep. Curr. Biol. 18, R670-R679.

Andretic, R., van Swinderen, B., and Greenspan, R.J. (2005). Dopaminergic modulation of arousal in Drosophila. Curr. Biol. 15, 1165-1175.

Asahina, K., Watanabe, K., Duistermars, B.J., Hoopfer, E., González, C.R., Eyjólfsdóttir, E.A., Perona, P., and Anderson, D.J. (2014). Tachykinin-Expressing Neurons Control Male-Specific Aggressive Arousal in Drosophila. Cell 156, 221-235.

Baines, R.A., Uhler, J.P., Thompson, A., Sweeney, S.T., and Bate, M. (2001). Altered electrical properties in Drosophila neurons developing without synaptic transmission. J. Neurosci. 21, 1523-1531.

Banerjee, S., Lee, J., Venkatesh, K., Wu, C.-F., and Hasan, G. (2004). Loss of flight and associated neuronal rhythmicity in inositol 1,4,5-trisphosphate receptor mutants of Drosophila. J. Neurosci. 24, 7869-7878.

Barnes, N.M., and Sharp, T. (1999). A review of central 5-HT receptors and their function. Neuropharmacology 38, 1083-1152.

Basler, K., and Struhl, G. (1994). Compartment boundaries and the control of Drosophila limb pattern by hedgehog protein. Nature 368, 208-214.

Becnel, J., Johnson, O., Luo, J., Nässel, D.R., and Nichols, C.D. (2011). The serotonin 5-HT7Dro receptor is expressed in the brain of Drosophila, and is essential for normal courtship and mating. PLoS ONE 6, e20800.

Benzer, S. (1967). BEHAVIORAL MUTANTS OF Drosophila ISOLATED BY COUNTERCURRENT DISTRIBUTION. Proc. Natl. Acad. Sci. U.S.A. 58, 1112-1119. 
Berlucchi, G. (1997). One or many arousal systems? Reflections on some of Giuseppe Moruzzi's foresights and insights about the intrinsic regulation of brain activity. Arch. Ital. Biol. 135, 5-14.

Bianchi, D., Marasco, A., Limongiello, A., Marchetti, C., Marie, H., Tirozzi, B., and Migliore, M. (2012). On the mechanisms underlying the depolarization block in the spiking dynamics of CA1 pyramidal neurons. J. Comput. Neurosci. 33, 207-225.

Blenau, W., and Thamm, M. (2011). Distribution of serotonin (5-HT) and its receptors in the insect brain with focus on the mushroom bodies: lessons from Drosophila melanogaster and Apis mellifera. Arthropod. Struct. Dev. 40, 381-394.

Blenau, W., Thamm, M., and Baumann, A. (2013). Serotonin in Insects: Distribution, Biosynthesis, Uptake, Inactivation, Receptors, Functions and Implications for Human Health. Hauppauge, NY: Nova Science Publishers. 1-26.

Blundell, J.E. (1984). Serotonin and appetite. Neuropharmacology 23, 1537-1551.

Bohm, R.A., Welch, W.P., Goodnight, L.K., Cox, L.W., Henry, L.G., Gunter, T.C., Bao, H., and Zhang, B. (2010). A genetic mosaic approach for neural circuit mapping in Drosophila. Proc. Natl. Acad. Sci. U.S.A. 107, 16378-16383.

Borbély, A.A., and Achermann, P. (1999). Sleep homeostasis and models of sleep regulation. J. Biol. Rhythms 14, 557-568.

Brand, A.H., and Perrimon, N. (1993). Targeted gene expression as a means of altering cell fates and generating dominant phenotypes. Development 118, 401-415.

Brent, R., and Ptashne, M. (1985). A eukaryotic transcriptional activator bearing the DNA specificity of a prokaryotic repressor. Cell 43, 729-736.

Brewerton, T.D. (1995). Toward a unified theory of serotonin dysregulation in eating and related disorders. Psychoneuroendocrinology 20, 561-590.

Chen, A., Ng, F., Lebestky, T., Grygoruk, A., Djapri, C., Lawal, H.O., Zaveri, H.A., Mehanzel, F., Najibi, R., Seidman, G., et al. (2013). Dispensable, redundant, complementary, and cooperative roles of dopamine, octopamine, and serotonin in Drosophila melanogaster. Genetics 193, 159176. 
Cirelli, C., and Bushey, D. (2008). Sleep and wakefulness in Drosophila melanogaster. Ann. N. Y. Acad. Sci. 1129, 323-329.

Clark, M.C., Dever, T.E., Dever, J.J., Xu, P., Rehder, V., Sosa, M.A., and Baro, D.J. (2004). Arthropod 5-HT2 receptors: a neurohormonal receptor in decapod crustaceans that displays agonist independent activity resulting from an evolutionary alteration to the DRY motif. J. Neurosci. 24, 3421-3435.

Clyne, J.D., and Miesenböck, G. (2008). Sex-specific control and tuning of the pattern generator for courtship song in Drosophila. Cell 133, 354-363.

Colas, J.F., Launay, J.M., Kellermann, O., Rosay, P., and Maroteaux, L. (1995). Drosophila 5-HT2 serotonin receptor: coexpression with fushi-tarazu during segmentation. Proc. Natl. Acad. Sci. U.S.A. 92, 5441-5445.

Coleman, C.M., and Neckameyer, W.S. (2005). Serotonin synthesis by two distinct enzymes in Drosophila melanogaster. Arch. Insect Biochem. Physiol. 59, 12-31.

Coull, J.T. (1998). Neural correlates of attention and arousal: insights from electrophysiology, functional neuroimaging and psychopharmacology. Prog. Neurobiol. 55, 343-361.

Crocker, A., Shahidullah, M., Levitan, I.B., and Sehgal, A. (2010). Identification of a neural circuit that underlies the effects of octopamine on sleep:wake behavior. Neuron 65, 670-681.

Dasari, S., Viele, K., Turner, A.C., and Cooper, R.L. (2007). Influence of PCPA and MDMA (ecstasy) on physiology, development and behavior in Drosophila melanogaster. Eur. J. Neurosci. 26, 424-438.

Daw, N.D., Kakade, S., and Dayan, P. (2002). Opponent interactions between serotonin and dopamine. Neural Netw. 15, 603-616.

Deakin, J.F., and Graeff, F.G. (1991). 5-HT and mechanisms of defence. Author's response. J. Psychopharmacol. (Oxford) 5, 339-341.

Depue, R.A., and Spoont, M.R. (1986). Conceptualizing a serotonin trait. A behavioral dimension of constraint. Ann. N. Y. Acad. Sci. 487, 47-62.

Devidze, N., Lee, A.W., Zhou, J., and Pfaff, D.W. (2006). CNS arousal mechanisms bearing on sex and other biologically regulated behaviors. Physiol. Behav. 88, 283-293. 
Dhaka, A., Viswanath, V., and Patapoutian, A. (2006). Trp ion channels and temperature sensation. Annu. Rev. Neurosci. 29, 135-161.

Dierick, H.A., and Greenspan, R.J. (2007). Serotonin and neuropeptide F have opposite modulatory effects on fly aggression. Nat. Genet. 39, 678-682.

Dubnau, J., Chiang, A.-S., Grady, L., Barditch, J., Gossweiler, S., McNeil, J., Smith, P., Buldoc, F., Scott, R., Certa, U., et al. (2003). The staufen/pumilio Pathway Is Involved in Drosophila LongTerm Memory. Current Biology 13, 286-296.

Dunbar, M.J., Tran, M.A., and Whelan, P.J. (2010). Endogenous extracellular serotonin modulates the spinal locomotor network of the neonatal mouse. J. Physiol. (Lond.) 588, 139156.

Fiala, A. (2007). Olfaction and olfactory learning in Drosophila: recent progress. Curr. Opin. Neurobiol. 17, 720-726.

Fiala, A., Spall, T., Diegelmann, S., Eisermann, B., Sachse, S., Devaud, J.-M., Buchner, E., and Galizia, C.G. (2002). Genetically expressed cameleon in Drosophila melanogaster is used to visualize olfactory information in projection neurons. Curr. Biol. 12, 1877-1884.

Fischer, J.A., Giniger, E., Maniatis, T., and Ptashne, M. (1988). GAL4 activates transcription in Drosophila. Nature 332, 853-856.

Flavell, S.W., Pokala, N., Macosko, E.Z., Albrecht, D.R., Larsch, J., and Bargmann, C.I. (2013). Serotonin and the neuropeptide PDF initiate and extend opposing behavioral states in $\mathrm{C}$. elegans. Cell 154, 1023-1035.

Flood, T.F., Gorczyca, M., White, B.H., Ito, K., and Yoshihara, M. (2013). A large-scale behavioral screen to identify neurons controlling motor programs in the Drosophila brain. G3 (Bethesda) 3, 1629-1637.

Fox, J.H., and Lowry, C.A. (2013). Corticotropin-releasing factor-related peptides, serotonergic systems, and emotional behavior. Front Neurosci 7, 169.

Friggi-Grelin, F., Coulom, H., Meller, M., Gomez, D., Hirsh, J., and Birman, S. (2003). Targeted gene expression in Drosophila dopaminergic cells using regulatory sequences from tyrosine hydroxylase. J. Neurobiol. 54, 618-627. 
Funk, N., Becker, S., Huber, S., Brunner, M., and Buchner, E. (2004). Targeted mutagenesis of the Sap47 gene of Drosophila: Flies lacking the synapse associated protein of $47 \mathrm{kDa}$ are viable and fertile. BMC Neurosci. 5, 16.

Gasbarri, A., and Pompili, A. (2014). Serotonergic 5-HT7 receptors and cognition. Rev Neurosci.

Gaspar, P., Cases, O., and Maroteaux, L. (2003). The developmental role of serotonin: news from mouse molecular genetics. Nat. Rev. Neurosci. 4, 1002-1012.

Gasque, G., Conway, S., Huang, J., Rao, Y., and Vosshall, L.B. (2013). Small molecule drug screening in Drosophila identifies the $5 \mathrm{HT} 2 \mathrm{~A}$ receptor as a feeding modulation target. Sci Rep. 3, srep02120.

Gillette, R. (2006). Evolution and function in serotonergic systems. Integr. Comp. Biol. 46, 838846.

Goldman, T.D., and Arbeitman, M.N. (2007). Genomic and functional studies of Drosophila sex hierarchy regulated gene expression in adult head and nervous system tissues. PLoS Genet. 3, e216.

Golic, K.G., and Lindquist, S. (1989). The FLP recombinase of yeast catalyzes site-specific recombination in the Drosophila genome. Cell 59, 499-509.

Gordon, M.D., and Scott, K. (2009). Motor control in a Drosophila taste circuit. Neuron 61, 373384.

Groth, A.C., Fish, M., Nusse, R., and Calos, M.P. (2004). Construction of Transgenic Drosophila by Using the Site-Specific Integrase From Phage $\phi C 31$. Genetics 166, 1775-1782.

Guo, J.-D., and Rainnie, D.G. (2010). Presynaptic 5-HT(1B) receptor-mediated serotonergic inhibition of glutamate transmission in the bed nucleus of the stria terminalis. Neuroscience 165, 1390-1401.

Haga, N., Mizumoto, A., Satoh, M., Mochiki, E., Mizusawa, F., Ohshima, K., and Itoh, Z. (1996). Role of endogenous 5-hydroxytryptamine in the regulation of gastric contractions by motilin in dogs. Am. J. Physiol. 270, G20-28. 
Hale, M.W., Shekhar, A., and Lowry, C.A. (2012). Stress-related serotonergic systems: implications for symptomatology of anxiety and affective disorders. Cell. Mol. Neurobiol. 32, 695-708.

Halford, J.C., and Blundell, J.E. (1996). The 5-HT1B receptor agonist CP-94,253 reduces food intake and preserves the behavioural satiety sequence. Physiol. Behav. 60, 933-939.

Haller, J. (2013). The neurobiology of abnormal manifestations of aggression--a review of hypothalamic mechanisms in cats, rodents, and humans. Brain Res. Bull. 93, 97-109.

Hamada, F.N., Rosenzweig, M., Kang, K., Pulver, S.R., Ghezzi, A., Jegla, T.J., and Garrity, P.A. (2008). An internal thermal sensor controlling temperature preference in Drosophila. Nature 454, 217-220.

Hansen, S., and Ross, S.B. (1983). Role of descending monoaminergic neurons in the control of sexual behavior: effects of intrathecal infusions of 6-hydroxydopamine and 5,7dihydroxytryptamine. Brain Res. 268, 285-290.

HEBB, D.O. (1955). Drives and the C.N.S. (conceptual nervous system). Psychol. Rev. 62, 243254.

Hendricks, J.C., Finn, S.M., Panckeri, K.A., Chavkin, J., Williams, J.A., Sehgal, A., and Pack, A.I. (2000). Rest in Drosophila is a sleep-like state. Neuron 25, 129-138.

Hetey, L., Kudrin, V.S., Shemanow, A.Y., Rayevsky, K.S., and Oelssner, W. (1985). Presynaptic dopamine and serotonin receptors modulating tyrosine hydroxylase activity in synaptosomes of the nucleus accumbens of rats. Eur. J. Pharmacol. 113, 1-10.

Hildebrandt, T., Alfano, L., Tricamo, M., and Pfaff, D.W. (2010). Conceptualizing the role of estrogens and serotonin in the development and maintenance of bulimia nervosa. Clin. Psychol. Rev. 30, 655-668.

Huber, R., Hill, S.L., Holladay, C., Biesiadecki, M., Tononi, G., and Cirelli, C. (2004). Sleep homeostasis in Drosophila melanogaster. Sleep 27, 628-639.

Ikemoto, S., and Panksepp, J. (1999). The role of nucleus accumbens dopamine in motivated behavior: a unifying interpretation with special reference to reward-seeking. Brain Res. Brain Res. Rev. 31, 6-41. 
Inagaki, H.K., Jung, Y., Hoopfer, E.D., Wong, A.M., Mishra, N., Lin, J.Y., Tsien, R.Y., and Anderson, D.J. (2014). Optogenetic control of Drosophila using a red-shifted channelrhodopsin reveals experience-dependent influences on courtship. Nat. Methods 11, 325-332.

Inagaki, H.K., Kamikouchi, A., and Ito, K. (2010). Methods for quantifying simple gravity sensing in Drosophila melanogaster. Nat Protoc 5, 20-25.

Jacobs, B.L., and Fornal, C.A. (1999). Activity of serotonergic neurons in behaving animals. Neuropsychopharmacology 21, 9S-15S.

Jenett, A., Rubin, G.M., Ngo, T.-T.B., Shepherd, D., Murphy, C., Dionne, H., Pfeiffer, B.D., Cavallaro, A., Hall, D., Jeter, J., et al. (2012). A GAL4-driver line resource for Drosophila neurobiology. Cell Rep. 2, 991-1001.

Jha, S., Rajendran, R., Davda, J., and Vaidya, V.A. (2006). Selective serotonin depletion does not regulate hippocampal neurogenesis in the adult rat brain: differential effects of $p$ chlorophenylalanine and 5,7-dihydroxytryptamine. Brain Res. 1075, 48-59.

Jing, J., Gillette, R., and Weiss, K.R. (2009). Evolving concepts of arousal: insights from simple model systems. Rev. Neurosci. 20, 405-427.

Kamyshev, N.G., Smirnova, G.P., Savvateeva, E.V., Medvedeva, A.V., and Ponomarenko, V.V. (1983). The influence of serotonin and p-chlorophenylalanine on locomotor activity of Drosophila melanogaster. Pharmacol. Biochem. Behav. 18, 677-681.

Kapur, S., and Remington, G. (1996). Serotonin-dopamine interaction and its relevance to schizophrenia. Am. J. Psychiatry 153, 466-476.

Kayser, M.S., Yue, Z., and Sehgal, A. (2014). A critical period of sleep for development of courtship circuitry and behavior in Drosophila. Science 344, 269-274.

Kitamoto, T. (2001). Conditional modification of behavior in Drosophila by targeted expression of a temperature-sensitive shibire allele in defined neurons. J. Neurobiol. 47, 81-92.

Koe, B.K., and Weissman, A. (1966). p-Chlorophenylalanine: a specific depletor of brain serotonin. J. Pharmacol. Exp. Ther. 154, 499-516.

Kume, K., Kume, S., Park, S.K., Hirsh, J., and Jackson, F.R. (2005). Dopamine is a regulator of arousal in the fruit fly. J. Neurosci. 25, 7377-7384. 
Lai, S.-L., and Lee, T. (2006). Genetic mosaic with dual binary transcriptional systems in Drosophila. Nat. Neurosci. 9, 703-709.

Laughon, A., and Gesteland, R.F. (1984). Primary structure of the Saccharomyces cerevisiae GAL4 gene. Mol. Cell. Biol. 4, 260-267.

Lebestky, T., Chang, J.-S.C., Dankert, H., Zelnik, L., Kim, Y.-C., Han, K.-A., Wolf, F.W., Perona, P., and Anderson, D.J. (2009). Two different forms of arousal in Drosophila are oppositely regulated by the dopamine D1 receptor ortholog DopR via distinct neural circuits. Neuron 64, 522-536.

Lee, P.-T., Lin, H.-W., Chang, Y.-H., Fu, T.-F., Dubnau, J., Hirsh, J., Lee, T., and Chiang, A.-S. (2011). Serotonin-mushroom body circuit modulating the formation of anesthesia-resistant memory in Drosophila. PNAS 108, 13794-13799.

Lee, Y., Lee, Y., Lee, J., Bang, S., Hyun, S., Kang, J., Hong, S.-T., Bae, E., Kaang, B.-K., and Kim, J. (2005). Pyrexia is a new thermal transient receptor potential channel endowing tolerance to high temperatures in Drosophila melanogaster. Nat. Genet. 37, 305-310.

Lefkimmiatis, K., Moyer, M.P., Curci, S., and Hofer, A.M. (2009). "cAMP sponge": a buffer for cyclic adenosine 3', 5'-monophosphate. PLoS ONE 4, e7649.

Lindquist, S. (1986). The heat-shock response. Annu. Rev. Biochem. 55, 1151-1191.

Liu, Q., Liu, S., Kodama, L., Driscoll, M.R., and Wu, M.N. (2012). Two dopaminergic neurons signal to the dorsal fan-shaped body to promote wakefulness in Drosophila. Curr. Biol. 22, 2114-2123.

Lowry, C.A., Hale, M.W., Evans, A.K., Heerkens, J., Staub, D.R., Gasser, P.J., and Shekhar, A. (2008). Serotonergic systems, anxiety, and affective disorder: focus on the dorsomedial part of the dorsal raphe nucleus. Ann. N. Y. Acad. Sci. 1148, 86-94.

Lowry, C.A., Johnson, P.L., Hay-Schmidt, A., Mikkelsen, J., and Shekhar, A. (2005). Modulation of anxiety circuits by serotonergic systems. Stress 8, 233-246.

Lucki, I. (1998). The spectrum of behaviors influenced by serotonin. Biol. Psychiatry 44, 151162. 
Luo, J., Becnel, J., Nichols, C.D., and Nässel, D.R. (2012). Insulin-producing cells in the brain of adult Drosophila are regulated by the serotonin 5-HT1A receptor. Cell. Mol. Life Sci. 69, 471484.

Mann, K., Gordon, M.D., and Scott, K. (2013). A Pair of Interneurons Influences the Choice between Feeding and Locomotion in Drosophila. Neuron 79, 754-765.

Marin, E.C., Jefferis, G.S.X.E., Komiyama, T., Zhu, H., and Luo, L. (2002). Representation of the Glomerular Olfactory Map in the Drosophila Brain. Cell 109, 243-255.

McGuire, S.E., Mao, Z., and Davis, R.L. (2004b). Spatiotemporal gene expression targeting with the TARGET and gene-switch systems in Drosophila. Sci. STKE 2004, pl6.

McGuire, S.E., Roman, G., and Davis, R.L. (2004a). Gene expression systems in Drosophila: a synthesis of time and space. Trends Genet. 20, 384-391.

Monti, J.M. (2011). Serotonin control of sleep-wake behavior. Sleep Med Rev 15, 269-281.

Myoga, H., Nonaka, S., Matsuyama, K., and Mori, S. (1995). Postnatal development of locomotor movements in normal and para-chlorophenylalanine-treated newborn rats. Neurosci. Res. 21, 211-221.

Nagel, G., Brauner, M., Liewald, J.F., Adeishvili, N., Bamberg, E., and Gottschalk, A. (2005). Light activation of channelrhodopsin-2 in excitable cells of Caenorhabditis elegans triggers rapid behavioral responses. Curr. Biol. 15, 2279-2284.

Neckameyer, W.S. (2010). A trophic role for serotonin in the development of a simple feeding circuit. Dev. Neurosci. 32, 217-237.

Neckameyer, W.S., and Bhatt, P. (2012). Neurotrophic actions of dopamine on the development of a serotonergic feeding circuit in Drosophila melanogaster. BMC Neurosci. 13, 26.

Neckameyer, W.S., Coleman, C.M., Eadie, S., and Goodwin, S.F. (2007). Compartmentalization of neuronal and peripheral serotonin synthesis in Drosophila melanogaster. Genes Brain Behav. $6,756-769$.

Nichols, C.D. (2007). 5-HT2 receptors in Drosophila are expressed in the brain and modulate aspects of circadian behaviors. Dev. Neurobiol. 67, 752-763. 
Nitabach, M.N., Blau, J., and Holmes, T.C. (2002). Electrical silencing of Drosophila pacemaker neurons stops the free-running circadian clock. Cell 109, 485-495.

Nitz, D.A., van Swinderen, B., Tononi, G., and Greenspan, R.J. (2002). Electrophysiological correlates of rest and activity in Drosophila melanogaster. Curr. Biol. 12, 1934-1940.

Okusawa, S., Kohsaka, H., and Nose, A. (2014). Serotonin and downstream leucokinin neurons modulate larval turning behavior in Drosophila. J. Neurosci. 34, 2544-2558.

Parisky, K.M., Agosto, J., Pulver, S.R., Shang, Y., Kuklin, E., Hodge, J.J.L., Kang, K., Kang, K., Liu, X., Garrity, P.A., et al. (2008). PDF cells are a GABA-responsive wake-promoting component of the Drosophila sleep circuit. Neuron 60, 672-682.

Park, J., Lee, S.B., Lee, S., Kim, Y., Song, S., Kim, S., Bae, E., Kim, J., Shong, M., Kim, J.-M., et al. (2006a). Mitochondrial dysfunction in Drosophila PINK1 mutants is complemented by parkin. Nature 441, 1157-1161.

Park, S.K., George, R., Cai, Y., Chang, H.Y., Krantz, D.E., Friggi-Grelin, F., Birman, S., and Hirsh, J. (2006b). Cell-type-specific limitation on in vivo serotonin storage following ectopic expression of the Drosophila serotonin transporter, dSERT. J. Neurobiol. 66, 452-462.

Parkes, T.L., Elia, A.J., Dickinson, D., Hilliker, A.J., Phillips, J.P., and Boulianne, G.L. (1998). Extension of Drosophila lifespan by overexpression of human SOD1 in motorneurons. Nat. Genet. 19, 171-174.

Patapoutian, A., Peier, A.M., Story, G.M., and Viswanath, V. (2003). ThermoTRP channels and beyond: mechanisms of temperature sensation. Nat. Rev. Neurosci. 4, 529-539.

Paul, E.D., Hale, M.W., Lukkes, J.L., Valentine, M.J., Sarchet, D.M., and Lowry, C.A. (2011). Repeated social defeat increases reactive emotional coping behavior and alters functional responses in serotonergic neurons in the rat dorsal raphe nucleus. Physiol. Behav. 104, 272282.

Pech, U., Pooryasin, A., Birman, S., and Fiala, A. (2013). Localization of the contacts between Kenyon cells and aminergic neurons in the Drosophila melanogaster brain using SplitGFP reconstitution. J. Comp. Neurol. 521, 3992-4026. 
Peru Y Colón de Portugal, R.L., Ojelade, S.A., Penninti, P.S., Dove, R.J., Nye, M.J., Acevedo, S.F., Lopez, A., Rodan, A.R., and Rothenfluh, A. (2013). Long-lasting, experience-dependent alcohol preference in Drosophila. Addict. Biol. 19, 392-401.

Pfaff, D. (2005b). Hormone-driven mechanisms in the central nervous system facilitate the analysis of mammalian behaviours. J. Endocrinol. 184, 447-453.

Pfaff, D., Ribeiro, A., Matthews, J., and Kow, L.-M. (2008). Concepts and mechanisms of generalized central nervous system arousal. Ann. N. Y. Acad. Sci. 1129, 11-25.

Pfaff, DW. (2005a). Brain arousal and information theory: neural and genetic mechanisms. Cambridge, MA:Harvard University Press.

Pfeiffenberger, C., Lear, B.C., Keegan, K.P., and Allada, R. (2010). Locomotor activity level monitoring using the Drosophila Activity Monitoring (DAM) System. Cold Spring Harb Protoc 2010, pdb.prot5518.

Pfeiffer, B.D., Ngo, T.-T.B., Hibbard, K.L., Murphy, C., Jenett, A., Truman, J.W., and Rubin, G.M. (2010). Refinement of tools for targeted gene expression in Drosophila. Genetics 186, 735-755.

Phelps, C.B., and Brand, A.H. (1998). Ectopic gene expression in Drosophila using GAL4 system. Methods 14, 367-379.

Pignoni, F., and Zipursky, S.L. (1997). Induction of Drosophila eye development by decapentaplegic. Development 124, 271-278.

Pulver, S.R., Pashkovski, S.L., Hornstein, N.J., Garrity, P.A., and Griffith, L.C. (2009). Temporal dynamics of neuronal activation by Channelrhodopsin-2 and TRPA1 determine behavioral output in Drosophila larvae. J. Neurophysiol. 101, 3075-3088.

Richardt, A., Kemme, T., Wagner, S., Schwarzer, D., Marahiel, M.A., and Hovemann, B.T. (2003). Ebony, a novel nonribosomal peptide synthetase for beta-alanine conjugation with biogenic amines in Drosophila. J. Biol. Chem. 278, 41160-41166.

Robbins, T.W. (1997). Arousal systems and attentional processes. Biol. Psychol. 45, 57-71.

Robbins, T.W., Granon, S., Muir, J.L., Durantou, F., Harrison, A., and Everitt, B.J. (1998). Neural systems underlying arousal and attention. Implications for drug abuse. Ann. N. Y. Acad. Sci. 846, 222-237. 
Rosen, S.C., Weiss, K.R., Goldstein, R.S., and Kupfermann, I. (1989). The role of a modulatory neuron in feeding and satiation in Aplysia: effects of lesioning of the serotonergic metacerebral cells. J. Neurosci. 9, 1562-1578.

Rosenzweig, M., Brennan, K.M., Tayler, T.D., Phelps, P.O., Patapoutian, A., and Garrity, P.A. (2005). The Drosophila ortholog of vertebrate TRPA1 regulates thermotaxis. Genes Dev. 19, 419-424.

Rubin, G.M., and Spradling, A.C. (1982). Genetic transformation of Drosophila with transposable element vectors. Science 218, 348-353.

Saper, C.B., Fuller, P.M., Pedersen, N.P., Lu, J., and Scammell, T.E. (2010). Sleep state switching. Neuron 68, 1023-1042.

Sasaki-Adams, D.M., and Kelley, A.E. (2001). Serotonin-dopamine interactions in the control of conditioned reinforcement and motor behavior. Neuropsychopharmacology 25, 440-452.

Saudou, F., Boschert, U., Amlaiky, N., Plassat, J.L., and Hen, R. (1992). A family of Drosophila serotonin receptors with distinct intracellular signalling properties and expression patterns. EMBO J. 11, 7-17.

Schindelin, J., Arganda-Carreras, I., Frise, E., Kaynig, V., Longair, M., Pietzsch, T., Preibisch, S., Rueden, C., Saalfeld, S., Schmid, B., et al. (2012). Fiji: an open-source platform for biologicalimage analysis. Nat. Methods 9, 676-682.

Schuldiner, O., Berdnik, D., Levy, J.M., Wu, J.S., Luginbuhl, D., Gontang, A.C., and Luo, L. (2008). piggyBac-based mosaic screen identifies a postmitotic function for cohesin in regulating developmental axon pruning. Dev. Cell 14, 227-238.

Seo, D., Patrick, C.J., and Kennealy, P.J. (2008). Role of Serotonin and Dopamine System Interactions in the Neurobiology of Impulsive Aggression and its Comorbidity with other Clinical Disorders. Aggress Violent Behav. 13, 383-395.

Shang, Y., Griffith, L.C., and Rosbash, M. (2008). Light-arousal and circadian photoreception circuits intersect at the large PDF cells of the Drosophila brain. Proc. Natl. Acad. Sci. U.S.A. 105, 19587-19594. 
Shaw, P.J., Cirelli, C., Greenspan, R.J., and Tononi, G. (2000). Correlates of sleep and waking in Drosophila melanogaster. Science 287, 1834-1837.

Silva, B., Goles, N.I., Varas, R., and Campusano, J.M. (2014). Serotonin receptors expressed in Drosophila mushroom bodies differentially modulate larval locomotion. PLoS ONE 9 ,

Sitaraman, D., LaFerriere, H., Birman, S., and Zars, T. (2012). Serotonin is critical for rewarded olfactory short-term memory in Drosophila. J. Neurogenet. 26, 238-244.

Sitaraman, D., Zars, M., Laferriere, H., Chen, Y.-C., Sable-Smith, A., Kitamoto, T., Rottinghaus, G.E., and Zars, T. (2008). Serotonin is necessary for place memory in Drosophila. Proc. Natl. Acad. Sci. U.S.A. 105, 5579-5584.

Sloley, B.D. (2004). Metabolism of monoamines in invertebrates: the relative importance of monoamine oxidase in different phyla. Neurotoxicology 25, 175-183.

Smith, C.M., Chua, B.E., Zhang, C., Walker, A.W., Haidar, M., Hawkes, D., Shabanpoor, F., Hossain, M.A., Wade, J.D., Rosengren, K.J., et al. (2014). Central injection of relaxin-3 receptor (RXFP3) antagonist peptides reduces motivated food seeking and consumption in C57BL/6J mice. Behav. Brain Res. 268, 217-126.

Spoont, M.R. (1992). Modulatory role of serotonin in neural information processing: implications for human psychopathology. Psychol. Bull. 112, 330-350.

St Johnston, D. (2002). The art and design of genetic screens: Drosophila melanogaster. Nat. Rev. Genet. 3, 176-188.

Steiger, H., Gauvin, L., Israël, M., Kin, N.M.K.N.Y., Young, S.N., and Roussin, J. (2004). Serotonin function, personality-trait variations, and childhood abuse in women with bulimia-spectrum eating disorders. J. Clin. Psychiatry 65, 830-837.

Sweeney, S.T., Broadie, K., Keane, J., Niemann, H., and O'Kane, C.J. (1995). Targeted expression of tetanus toxin light chain in Drosophila specifically eliminates synaptic transmission and causes behavioral defects. Neuron 14, 341-351.

Szüts, D., and Bienz, M. (2000). LexA chimeras reveal the function of Drosophila Fos as a context-dependent transcriptional activator. Proc. Natl. Acad. Sci. U.S.A. 97, 5351-5356. 
Tataroglu, O., and Emery, P. (2014). Studying circadian rhythms in Drosophila melanogaster. Methods.

Thum, A.S., Knapek, S., Rister, J., Dierichs-Schmitt, E., Heisenberg, M., and Tanimoto, H. (2006). Differential potencies of effector genes in adult Drosophila. J. Comp. Neurol. 498, 194-203.

Tracey, W.D., Jr, Wilson, R.I., Laurent, G., and Benzer, S. (2003). painless, a Drosophila gene essential for nociception. Cell 113, 261-273.

Ueno, T., Tomita, J., Tanimoto, H., Endo, K., Ito, K., Kume, S., and Kume, K. (2012). Identification of a dopamine pathway that regulates sleep and arousal in Drosophila. Nat. Neurosci. 15, 15161523.

Vallés, A.M., and White, K. (1988). Serotonin-containing neurons in Drosophila melanogaster: development and distribution. J. Comp. Neurol. 268, 414-428.

Van Swinderen, B., and Andretic, R. (2003). Arousal in Drosophila. Behav. Processes 64, 133144.

Van Swinderen, B., and Andretic, R. (2011). Dopamine in Drosophila: setting arousal thresholds in a miniature brain. Proc. Biol. Sci. 278, 906-913.

Vaysse, G., Galissié, M., and Corbière, M. (1988). Induced variation of serotonin in Drosophila melanogaster and its relation to learning performance. Journal of Comparative Psychology 102, 225-229.

Venken, K.J.T., Simpson, J.H., and Bellen, H.J. (2011). Genetic manipulation of genes and cells in the nervous system of the fruit fly. Neuron $72,202-230$.

Viswanath, V., Story, G.M., Peier, A.M., Petrus, M.J., Lee, V.M., Hwang, S.W., Patapoutian, A., and Jegla, T. (2003). Opposite thermosensor in fruitfly and mouse. Nature 423, 822-823.

Vogelstein, J.T., Park, Y., Ohyama, T., Kerr, R., Truman, J.W., Priebe, C.E., and Zlatic, M. (2014). Discovery of Brainwide Neural-Behavioral Maps via Multiscale Unsupervised Structure Learning. Science 1250298.

Wagh, D.A., Rasse, T.M., Asan, E., Hofbauer, A., Schwenkert, I., Dürrbeck, H., Buchner, S., Dabauvalle, M.-C., Schmidt, M., Qin, G., et al. (2006). Bruchpilot, a protein with homology to 
ELKS/CAST, is required for structural integrity and function of synaptic active zones in Drosophila. Neuron 49, 833-844.

Wing, J.P., Zhou, L., Schwartz, L.M., and Nambu, J.R. (1998). Distinct cell killing properties of the Drosophila reaper, head involution defective, and grim genes. Cell Death Differ. 5, 930-939.

Witz, P., Amlaiky, N., Plassat, J.L., Maroteaux, L., Borrelli, E., and Hen, R. (1990). Cloning and characterization of a Drosophila serotonin receptor that activates adenylate cyclase. Proc. Natl. Acad. Sci. U.S.A. 87, 8940-8944.

Wong, P.T., Feng, H., and Teo, W.L. (1995). Interaction of the dopaminergic and serotonergic systems in the rat striatum: effects of selective antagonists and uptake inhibitors. Neurosci. Res. 23, 115-119.

Wu, C.-L., Shih, M.-F.M., Lee, P.-T., and Chiang, A.-S. (2013). An Octopamine-Mushroom Body Circuit Modulates the Formation of Anesthesia-Resistant Memory in Drosophila. Current Biology 23, 2346-2354.

Yan, Z. (2002). Regulation of GABAergic inhibition by serotonin signaling in prefrontal cortex: molecular mechanisms and functional implications. Mol. Neurobiol. 26, 203-216.

Yu, J.Y., Kanai, M.I., Demir, E., Jefferis, G.S.X.E., and Dickson, B.J. (2010). Cellular Organization of the Neural Circuit that Drives Drosophila Courtship Behavior. Current Biology 20, 1602-1614.

Yuan, Q., Joiner, W.J., and Sehgal, A. (2006). A sleep-promoting role for the Drosophila serotonin receptor 1A. Curr. Biol. 16, 1051-1062.

Yuan, Q., Lin, F., Zheng, X., and Sehgal, A. (2005). Serotonin modulates circadian entrainment in Drosophila. Neuron 47, 115-127. 


\section{Abbreviations}

\begin{tabular}{|c|c|}
\hline$\%$ & percent \\
\hline${ }^{\circ} \mathrm{C}$ & degrees Celsius \\
\hline$\mu g$ & microgram \\
\hline$\mu l$ & microliter \\
\hline 5-HT & 5-Hydroxytryptamine \\
\hline ChR2 & channelrhodopsin2 \\
\hline cm & centimeter \\
\hline CNS & central nervous system \\
\hline COMT & catechol-O-methyl transferase \\
\hline Cy3 & cyanine dye 3 \\
\hline DDC & DOPA decarboxylase \\
\hline $\mathrm{ddH}_{2} \mathrm{O}$ & Double distilled water \\
\hline DNA & deoxyribonucleic acid \\
\hline DopR & D1-like dopamine receptor \\
\hline dORK & Drosophila open rectifier $\mathrm{K}^{+}$channel \\
\hline dSERT & Drosophila serotonin transporter \\
\hline dTRPA1 & Drosophila transient receptor potential channel A1 \\
\hline e.g. & exempli gratia \\
\hline FLP & flippase \\
\hline FRT & flippase recombination target sites \\
\hline g & gram \\
\hline
\end{tabular}




\begin{tabular}{ll} 
Gal4 & galactosidase 4 \\
Gal80 & galactose/lactose metabolism regulatory protein \\
GFP & green fluorescent protein \\
h & hour \\
hs & heat shock promoter \\
HZ & hertz \\
Kir2.1 & inward rectifying potassium channel \\
LexAop & LexA operator \\
M & molar \\
Mao & monoamine oxidase \\
MCCs & metacerebral cells \\
min & minute \\
mI & milliliter \\
NGS & normal goat serum \\
nSyb & thoracic ganglion \\
PBS & synaptobrevin \\
PCPA & Phosphate buffer saline \\
PCR & p-chlorophenylalanine \\
PFA & polymerase chain reaction \\
RFP & shrimp alkaline phosphatase \\
SA & red fluorescent protein \\
TG & second \\
\hline ts & sherature sensitive shibire \\
\hline
\end{tabular}




$\begin{array}{ll}\text { TNT } & \text { tetanus toxin } \\ \text { TPH } & \text { phenylalanine hydroxylase } \\ \text { TRH } & \text { tryptophan hydroxylase } \\ \text { TRP channel } & \text { transient receptor potential channel } \\ \text { tub } & \text { tubulin } \\ \text { UAS } & \text { upstream activation sequence } \\ \text { V } & \text { volt }\end{array}$




\section{Acknowledgments}

I would like to express my special appreciation and thanks to my supervisor Prof. Dr. André Fiala for all I have learned from him and for his continuous support in all stages of my PhD study. His guidance helped me throughout the time of my research and I appreciate his vast knowledge in many areas.

I would also like to thank the members of my thesis committee, Prof. Dr. Martin Göpfert and Prof. Dr. Julia Fischer, for their time and insightful comments during the thesis committee meetings.

I would like to appreciate DFG for funding this research project.

I also want to thank Dr. Annette Witt for her assistance and for her helpful suggestions for the statistical analysis.

I would like to thank my former and current lab members who made the lab a friendly environment for working: Abud, Carlotta, David, Jonas, Jutta, Mandy, Priyanka, Sandra, Shubham, Simon, Tom and Uli. Special thanks to Shubham for his help, especially with the evaluation of data from the activity and sleep experiment, to Uli and Tom for their advices and helpful discussions during my PhD and to Jutta for offering me help whenever I was in the time pressure. I am grateful to Uli, Shubham and David for being good friends and being beside me in the happy and sad moments of my life in Germany. I appreciate all the long evenings we spent working together in the lab.

I would like to express my gratitude to the GGNB for their organization and assistance during the last four years of $\mathrm{my} \mathrm{PhD}$ and for providing me the bridging fund for the last three months of my PhD work.

I am grateful to my closest friend, Tahereh, who was always kind, generous and supportive during the last eight years of my life. I would like to thank Mohammad for his presence and great support especially at the times of stress and to Reza, Rashin, Shayan, Nikolai and Ali for their friendship.

Last but not least, I would like to thank my mother, my father and my brothers for always being supportive and encouraging, for their continuous love and their trust in my decisions. Without them I could not have made it here. 
